\title{
Dissertation
}

\author{
in partial fulfilment of the requirements \\ for the degree "Doctor rerum naturalium (Dr. rer. nat)" \\ in the Molecular Biology Program \\ at the Georg August University Göttingen, \\ Faculty of Biology
}

\section{Exploration of cargo spectrum and NES patterns recognized by the exportin CRM1}

\author{
submitted by \\ Koray Kırlı \\ from Istanbul (Turkey)
}

Göttingen, September 2013 


\section{Members of the Thesis Committee}

Prof. Dr. Dirk Görlich

(Thesis mentor and referee)

Prof. Dr. Reinhard Jahn

(Co-referee)

Prof. Dr. Kai Tittmann

Additional Members of the Examination Board

Prof. Dr. Detlef Doenecke

Prof. Dr. Peter Rehling

Prof. Dr. Holger Stark
Max Planck Institute for Biophysical Chemistry

Dept. of Cellular Logistics

Göttingen, Germany

Max Planck Institute for Biophysical Chemistry

Dept. of Neurobiology

Göttingen, Germany

Department of Bioanalytics

Georg August University

Göttingen, Germany

Universitätsmedizin (UMG)

Georg August University

Göttingen, Germany

Dept. of Biochemistry II

Georg August University

Göttingen, Germany

Max Planck Institute for Biophysical Chemistry

3D Electron Cryo-Microscopy Research Group

Göttingen, Germany 
The research detailed in this thesis has been performed in the laboratory of Prof. Dr. Dirk Görlich at the Max-Planck-Institute for Biophysical Chemistry in the time from October 2009 to September 2013.

I hereby declare that I completed my thesis entitled

\section{"Exploration of cargo spectrum and NES patterns recognized by the exportin CRM1"}

independently and with no other sources and aids than quoted. This dissertation has not been submitted elsewhere for any academic award or qualification.

Koray Kırlı

Göttingen, September 2013 
This thesis is dedicated to the ones that suffered for a brighter future

A.I.K.

M.A.

M.Y.

A.C.

A.A.

E.S. 


\section{TABLE OF CONTENTS}

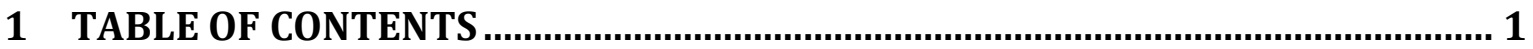

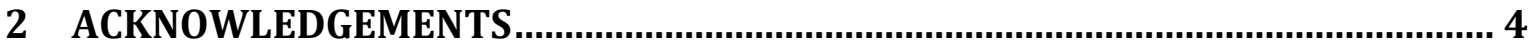

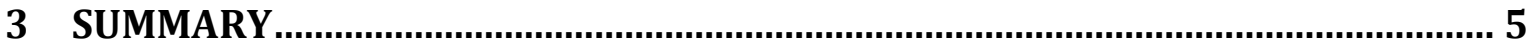

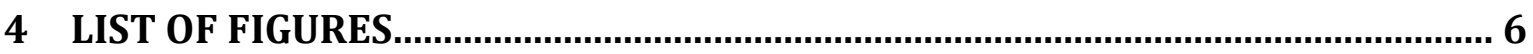

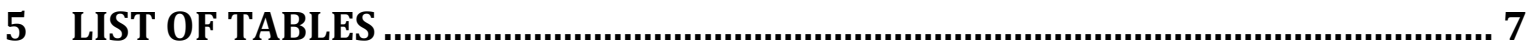

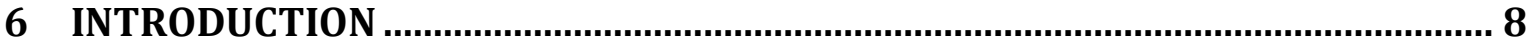

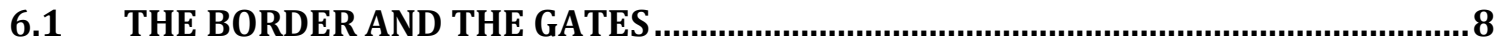

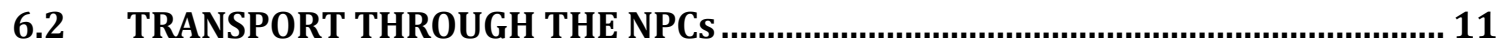

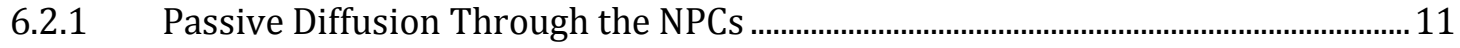

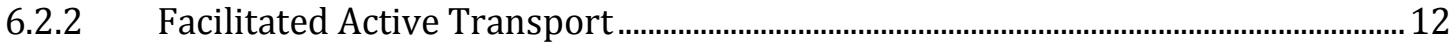

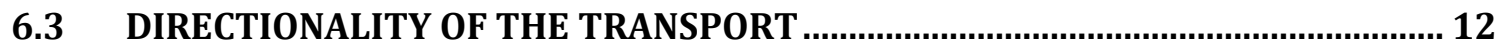

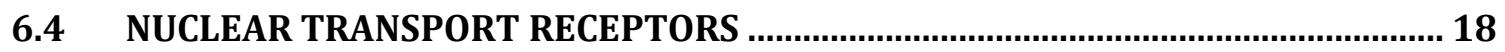

6.4.1 CRM1/Exportin 1 ................................................................................................. 20

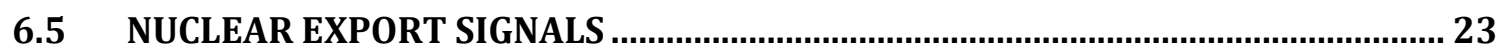

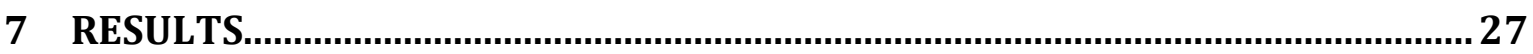

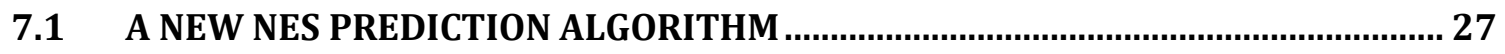

7.1.1 NES Consensus................................................................................................................ 28

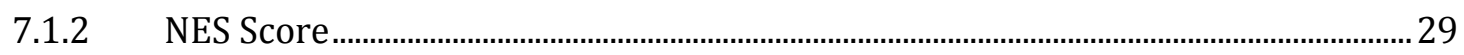

7.1.3 Disorder Propensities ............................................................................................. 30

7.1.4 Evaluation of PKI-type NES prediction ........................................................................ 31

7.1.5 Evaluation of REV-type NES prediction....................................................................... 35

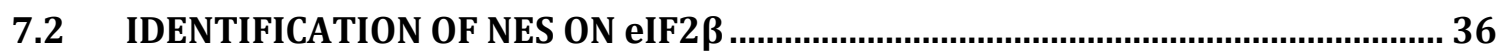

7.2.1 Prediction of eIF2 $\beta$ NES hits ........................................................................................... 36

7.2.2 Validation of eIF2 $\beta$ NES hit .......................................................................................... 36

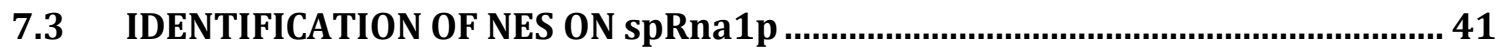

7.3.1 Previously suggested NESs are buried in the structure .............................................. 41

7.3.2 Prediction of spRna1p NES hits ..................................................................................... 42

7.3.3 Validation of spRna1p NES hit........................................................................................... 42

7.4 RANGTP DEPENDENT CRM1 BINDERS FROM CYTOSOLIC HELA EXTRACT ....... 49

7.4.1 CRM1 affinity chromatography of cytoplasmic HeLa extract...................................... 49 
7.4.2 CRM1 Affinity Chromatography for SILAC-Based Mass Spectrometry 50

7.4.3 Mass Spectrometry Analysis of SILAC CRM1 Affinity Chromatography Eluates 53

8 DISCUSSION.. 61

8.1 A NEW PREDICTION ALGORITHM FOR CRM1 DEPENDENT NESS 61

8.2 MASS SPECTROMETRY ANALYSIS OF HELA CRM1 CARGOES ............................... 65

9 OUTLOOK

10 MATERIAL AND METHODS

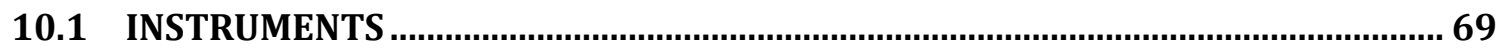

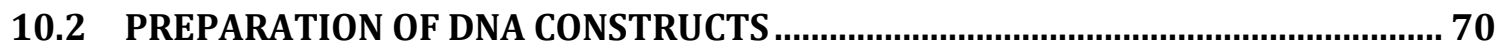

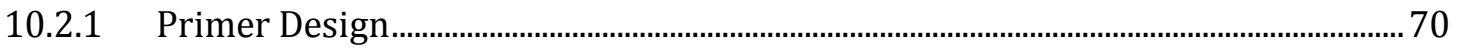

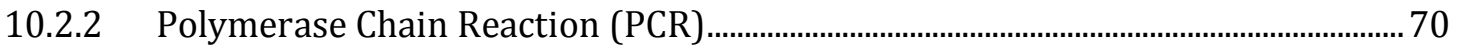

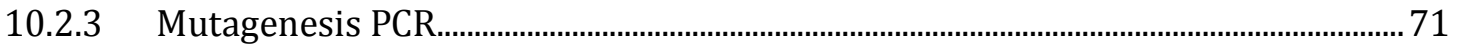

10.2.4 DNA Cleavage with Restriction Enzymes............................................................................ 73

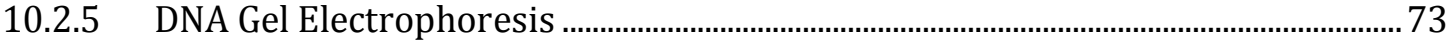

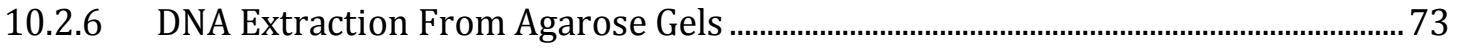

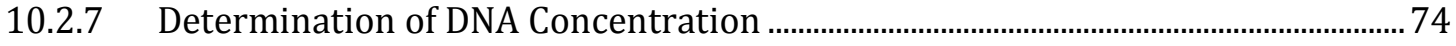

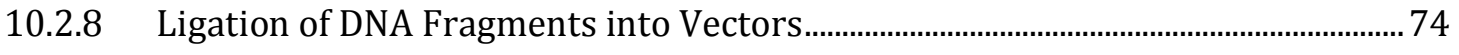

10.2.9 Electroporation of E. coli Cells ................................................................................... 74

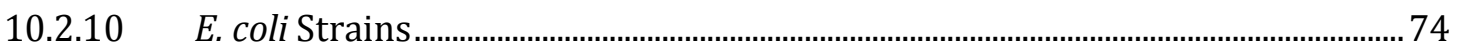

10.2.11 DNA Purification From E. coli Cultures ........................................................................... 75

10.2.12 Bacterial Expression Constructs............................................................................. 75

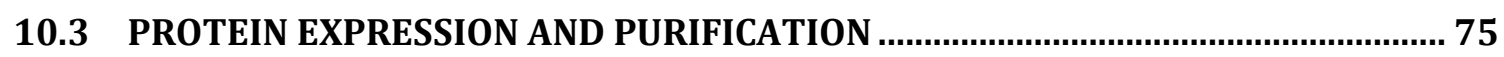

10.3.1 Native Protein Expression and Purification ………………………………………….... 75

10.3.2 Determination of Protein Concentrations ............................................................................ 77

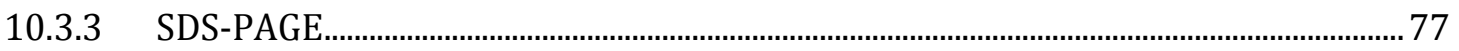

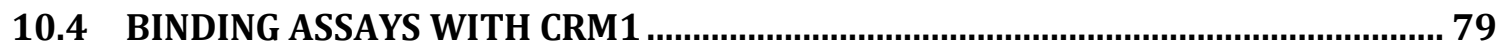

10.4.1 Binding Assays with Purified Components.........................................................................

10.5 PULL DOWN FROM CYTOPLASMIC EXTRACT WITH CRM1 …................................ 81

10.5.1 Preparation of Cytoplasmic Extracts................................................................................. 81

10.5.2 Preparation of Cytoplasmic SILAC HeLa Extracts .............................................................. 82

10.5.3 CRM1 Affinity Chromatography with Cytoplasmic HeLa Extracts ............................ 82

10.5.4 Mass Spectrometry Analysis of Elution Fractions.......................................................... 84

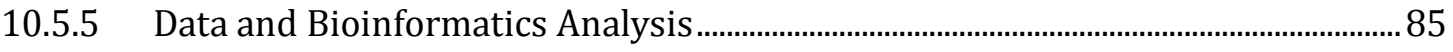

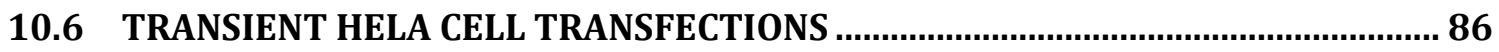

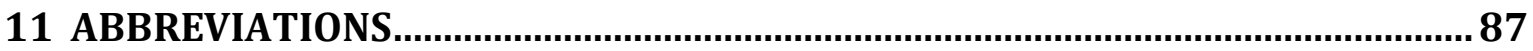




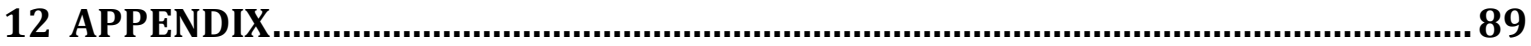

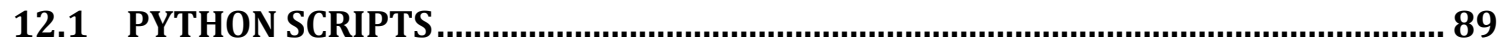

12.1.1 PKI-type NES Prediction .....................................................................................................

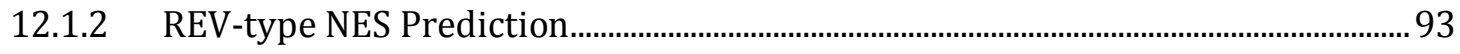

12.2 PROTEIN IDENTIFIERS OF THE DATA SETS.......................................................96

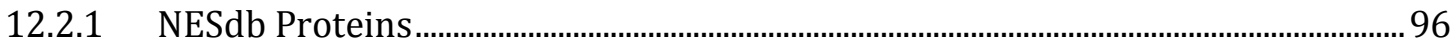

12.2.2 LMB Study Proteins ..............................................................................................................97

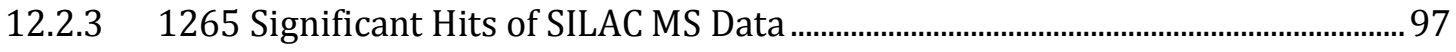

12.2.4 321 Proteins with a Predicted NES ………………………………………………...... 104

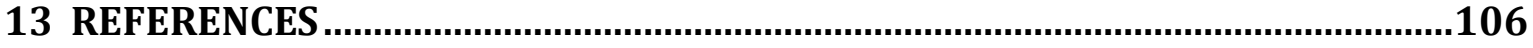




\section{ACKNOWLEDGEMENTS}

I would like to thank

Dirk Görlich, who has been a great mentor, for giving me the opportunity to work with him for my thesis. His enthusiasm for science amazed and motivated me through out my doctoral work.

Reinhard Jahn and Kai Tittmann for being part of my thesis committee and for their contributions with valuable comments and discussions.

Henning Urlaub, Samir Karaca, Uwe Plessmann and Monika Raabe for the mass spectrometric analysis.

Samir Karaca for his suggestions and contributions with SILAC based method, and data analysis.

Bastian Hülsmann and Matthias Samwer for the lengthy discussions that broadened by scientific interest, and also for their friendship that made it easy to deal with the down times.

Steffen Frey for being the "room of requirements" of the lab, with many reagents and advices, and answering many questions with a great patience.

Miroslav Nikolov for kindly providing cytoplasmic SILAC HeLa extracts.

Heinz-Jürgen Dehne, Heike Behr, and Jürgen Schünemann for helping me at different stages of my doctoral studies with their excellent technical support.

Gabriele Kopp and Uwe Hoffmann for making the lab a ready to use place, by taking care of many things that one does not easily recognize.

Aksana Labokha for her supervision in the first stages of my doctoral studies.

members of the lab for fruitful discussions during lab and literature seminars, and for the cheerful atmosphere in the lab.

Steffen Burkhardt and Kerstin Grüniger for administrative support,

Metin Aksu, Kevser Gençalp and Samir Karaca that constituted the small Turkish district of the lab with me, for long scientific and non-scientific discussions during our coffee breaks, and also for always keeping me one step closer to home.

my family for their continuous support through out my studies.

Finally, I would like to thank Sinem K. Saka who has been the main inspiration to me, and was always there to cheer me up. 


\section{SUMMARY}

The nuclear envelope (NE) subdivides eukaryotic cells into a nuclear and a cytoplasmic compartment, forcing material exchange between these two compartments to proceed through the nuclear pore complexes (NPCs). While proteins smaller than 30-40 kDa can passively diffuse through the NPCs, larger objects require nuclear transport receptors (NTRs) for efficient transport. NTRs have the privilege of facilitated NPC-passage; they bind transport cargoes and transfer them from one side of the NE to the other. NTRs can act as unidirectional cargo pumps, whereby they utilize the chemical potential of the nucleocytoplasmic RanGTP gradient with high nuclear and low cytoplasmic RanGTP levels.

CRM1 is a major, essential and highly conserved nuclear export receptor. It exports a great variety of cargoes from the nucleus to the cytoplasm. CRM1 also keeps e.g. several translation factors and RanGAP cytoplasmic. The latter is required for maintaining the nucleocytoplasmic RanGTP gradient. CRM1 recognizes many cargoes through so-called leucine-rich nuclear export signal (NES), sequences containing 4-5 hydrophobic residues in a 14-15 residues long stretch. Although NESs are described in the context of primary protein structure, a reliable NES prediction has been a challenge and failed, e.g. for elF $2 \beta$ and Rna1p (S.pombe RanGAP).

Here we present a new NES prediction algorithm based on the recent crystal structures of different NES sequences with CRM1. We classified NES two PKI-type and REV-type with two different consensus definitions. PKI-type NES were graded for CRM1 binding strength and additional filtering was applied with disorder prediction. The REV-type NES was a novel classification based on Rev protein NES, and we show that there are several other examples of this type of NES. The estimation power of the new prediction algorithm was shown on prediction of already known NESs as control, and it also was able to predict the NESs of human eIF2 $\beta$ and S.pombe Rna1p, which was also confirmed experimentally.

Another challenge had been the question of how many different cargo species are actually transported by CRM1. To address this, we optimized affinity chromatography on immobilized CRM1 and used it to retrieve RanGTP-dependent cargoes from a cytoplasmic HeLa extract. This analysis revealed hundreds of new CRM1 cargo candidates, which were further group into functional protein categories. Most of the ribosomal proteins are found in our dataset. Besides them, we find serine threonine kinases, ATP dependent helicases, spliceosomal proteins, translation initiation factors, actin regulators, and E3 ubiquitin ligases. Proteins of metabolic pathways, cell adhesion, phagosome, and proteasome are excluded from the data set. 


\section{LIST OF FIGURES}

Figure 6-1 First known drawing of cells and nuclei by Antonie van Leeuwenhoek, ................................. 8

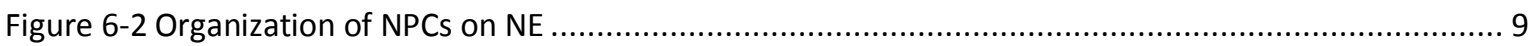

Figure $6-3$ Structural elements of vertebrate nucleoporins ......................................................... 10

Figure 6-4 Overview of active nucleocytoplasmic transport through NPCs ........................................ 14

Figure 6-5 Domain organization of RanGAP homologs .............................................................. 15

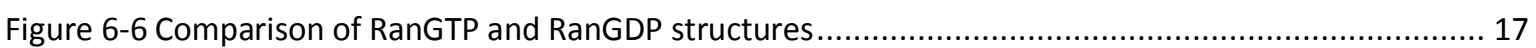

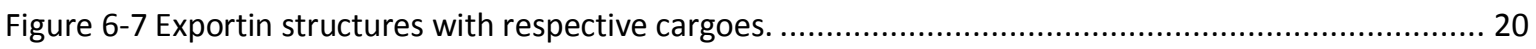

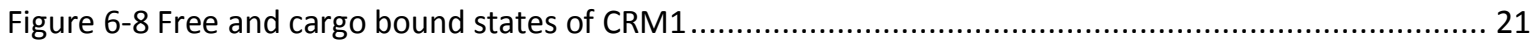

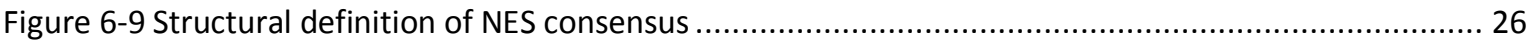

Figure 7-1 Regular expression for PKI-type NES pattern and an example NES ........................................ 28

Figure 7-2 Regular expression for REV-type NES pattern and an example NES..................................... 29

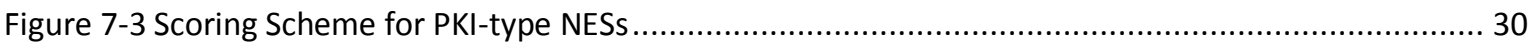

Figure 7-4 An output example from PKI- type NES prediction...................................................... 32

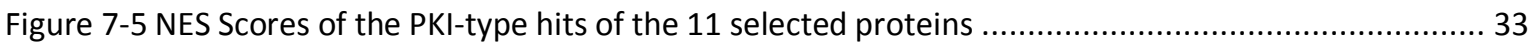

Figure 7-6 Aggregated disorder propensities of the PKI-type hits of the 11 selected proteins .................... 34

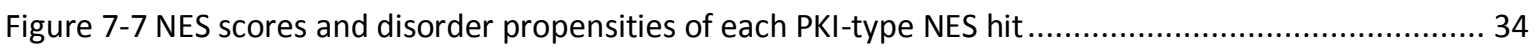

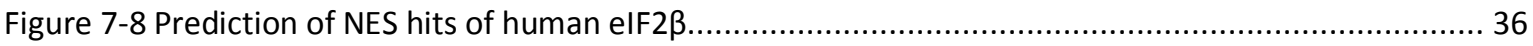

Figure 7-9 Alignment of hs elF2 $\beta$ protein sequence with vertebrate orthologs ................................... 37

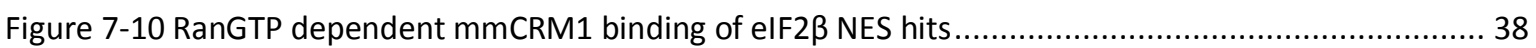

Figure 7-11 RanGTP dependent mmCRM1 binding of wild type and NES mutant of human elF2 $\beta \ldots \ldots \ldots \ldots . . . . .39$

Figure 7-12 spRna1p structure and previously suggested NESs ............................................... 41

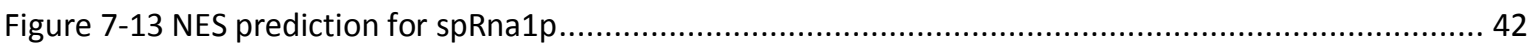

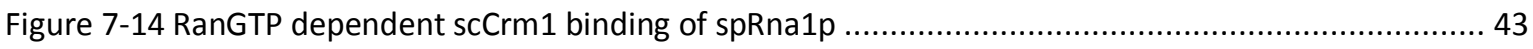

Figure 7-15 RanGTP dependent scCrm1 binding of spRna1p and point mutants ....................................45

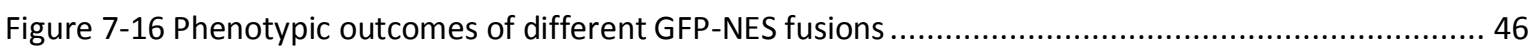

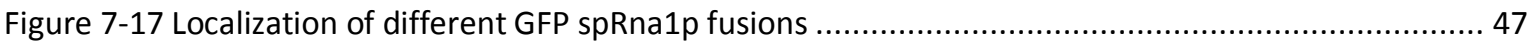

Figure 7-18 Salt sensitivity of RanGTP dependent mmCRM1 interaction of cargoes from HeLa S10 extract. 49 Figure 7-19 Distribution of protein ratios in heavy and light HeLa extracts ....................................... 51

Figure 7-20 CRM1 affinity chromatography of cytoplasmic HeLa extracts produced with SILAC method ..... 52

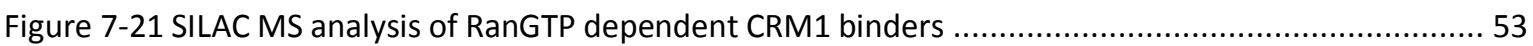

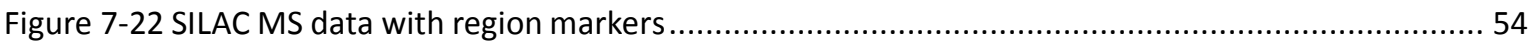

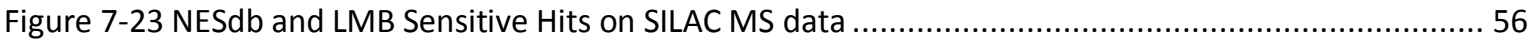

Figure 7-24 Protein groups that are over or under represented in MS data set................................... 57

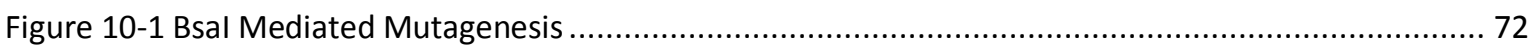




\section{LIST OF TABLES}

Table 6-1 Mammalian importins and selected cargos.................................................................. 19

Table 6-2 Mammalian exportins and selected cargoes .............................................................. 19

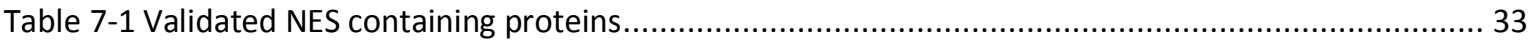

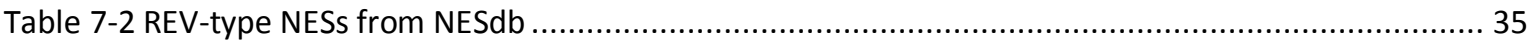

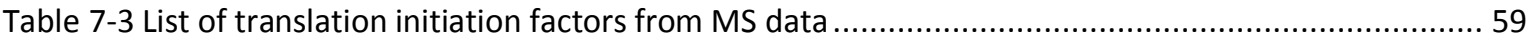

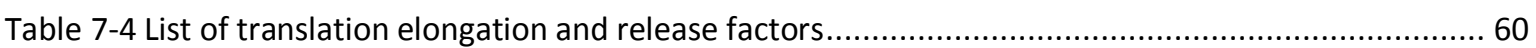

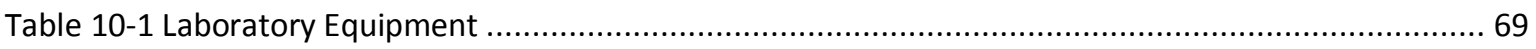

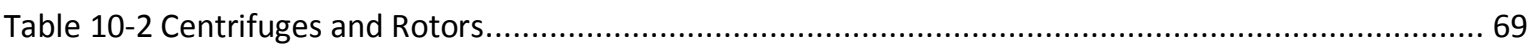

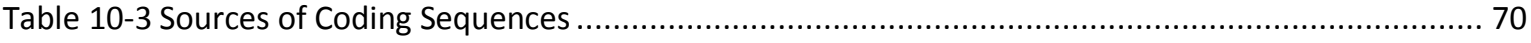

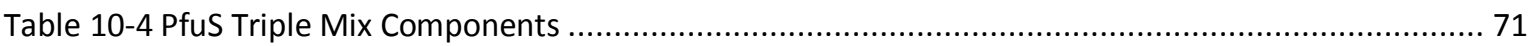

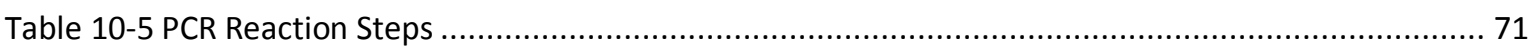

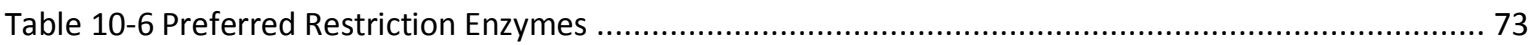

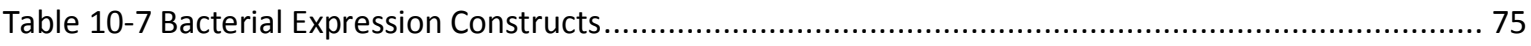

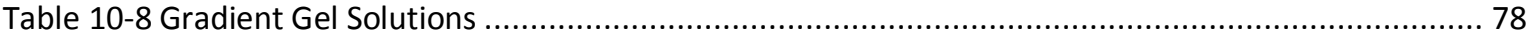

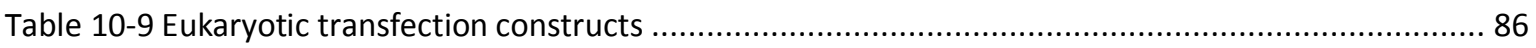

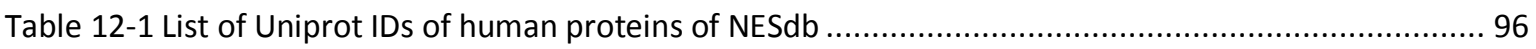

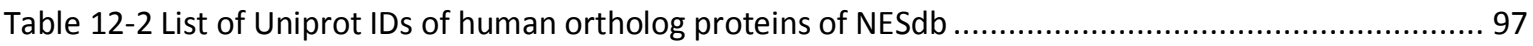

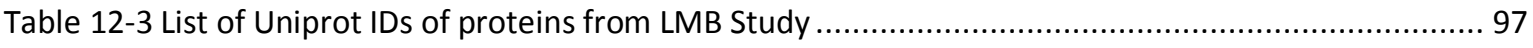

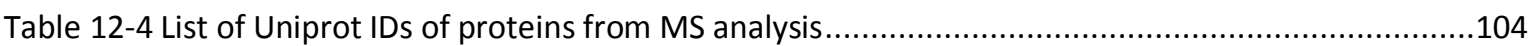

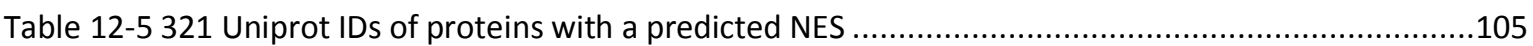




\section{INTRODUCTION}

The living things are interesting in many ways, and presumably their complex structure and organization is the most fascinating one for the scientists. Antonie van Leeuwenhoek's drawing of the salmon red blood cells marks one of the prominent moments of this fascination. Since the non-mammalian vertebrates retain their nuclei in the erythrocytes, by looking at them from his handcrafted microscope, he drew the first known figure of nucleus (Figure 6-1) (Delphis et al., 1719). It was not called 'nucleus' until Botanist Robert Brown coined the term in 1831 (Oliver, 1913). Since then many important aspects of the nucleus and its function have been revealed, which also brought many new questions.

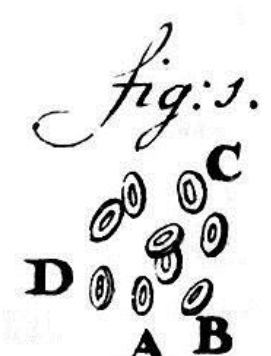

A B

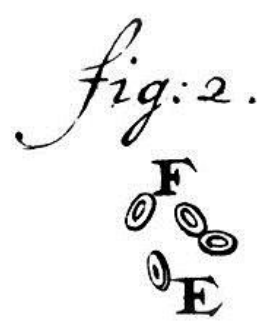

$\mathbf{E}$

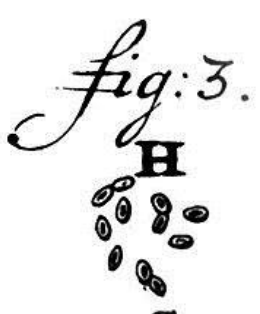

G

Figure 6-1 First known drawing of cells and nuclei by Antonie van Leeuwenhoek, 1719 (Delphis et al., 1719)

\subsection{THE BORDER AND THE GATES}

The hallmark of a eukaryotic cell is its compartmentalization into the nucleus and the cytoplasm, which are separated by the nuclear envelope (NE). The nuclear envelope is a double lipid bilayer that is continuous on the cytoplasmic side with the membrane of the endoplasmic reticulum (ER). The perinuclear space in between these membranes is also part of the ER lumen (Subramanian and Meyer, 1997). The evolution of a nucleus enabled the high-end regulation that was required for emergence of very complex multicellular organism (Gorlich and Kutay, 1999).

One of the advantages of the nuclear compartmentalization is that eukaryotes can handle a large amount of genetic material compared to prokaryotes. This enables more coding sequence and regulatory sequence to be accommodated in the genome. A second advantage is the control over the level and the timing of gene expression by regulating the nuclear localization of transcription factors (Kaffman and O'Shea, 1999).

The more striking evolution that comes with the nucleus is the compartmentalization of the cellular information processes. In bacteria DNA, RNA and ribosomes and other proteins take role in conversion of genetic information to functional proteins, and work side by side in a continuous process. Ribosomes start translating the mRNA as soon as its $5^{\prime}$ end is synthesized by the RNA 
polymerase. In eukaryotes, the NE spatially separates transcription and translation and necessitates localization of specific macromolecules to these compartments.

Since all proteins are produced in the cytoplasm, proteins necessary for DNA maintenance (e.g., histones), transcription (e.g., RNA polymerases), gene expression regulation (e.g., transcription factors) and many others required in the nucleus have to be imported (Bonner, 1975). On the other hand, transcribed and spliced mRNA, tRNA, assembled ribosomal subunits have to be exported to the cytoplasm. Segregation of macromolecules between the nucleus and the cytoplasm poses another challenge for higher eukaryotes. During cell division the NE breaks down and re-forms during telophase. At this point a high load of misplaced macromolecules has to be re-sorted. Thus nucleocytoplasmic transport has to be a very efficient and fast process to keep up with this load (Gorlich and Kutay, 1999).

The need for export and import of cargoes originates not only from the requirement of certain macromolecules and complexes in a specific compartment, but also from the necessity that certain activities should be temporarily or permanently be absent in either the nucleus or the cytoplasm. For example regulation of a gene's expression might depend on import of a specific transcription factor. This regulation necessitates the temporary exclusion of the transcription factor from the nucleus, which can be sustained by nuclear export as in the case of NF-KB/IKB $\alpha$ complexes (Huang et al., 2000).

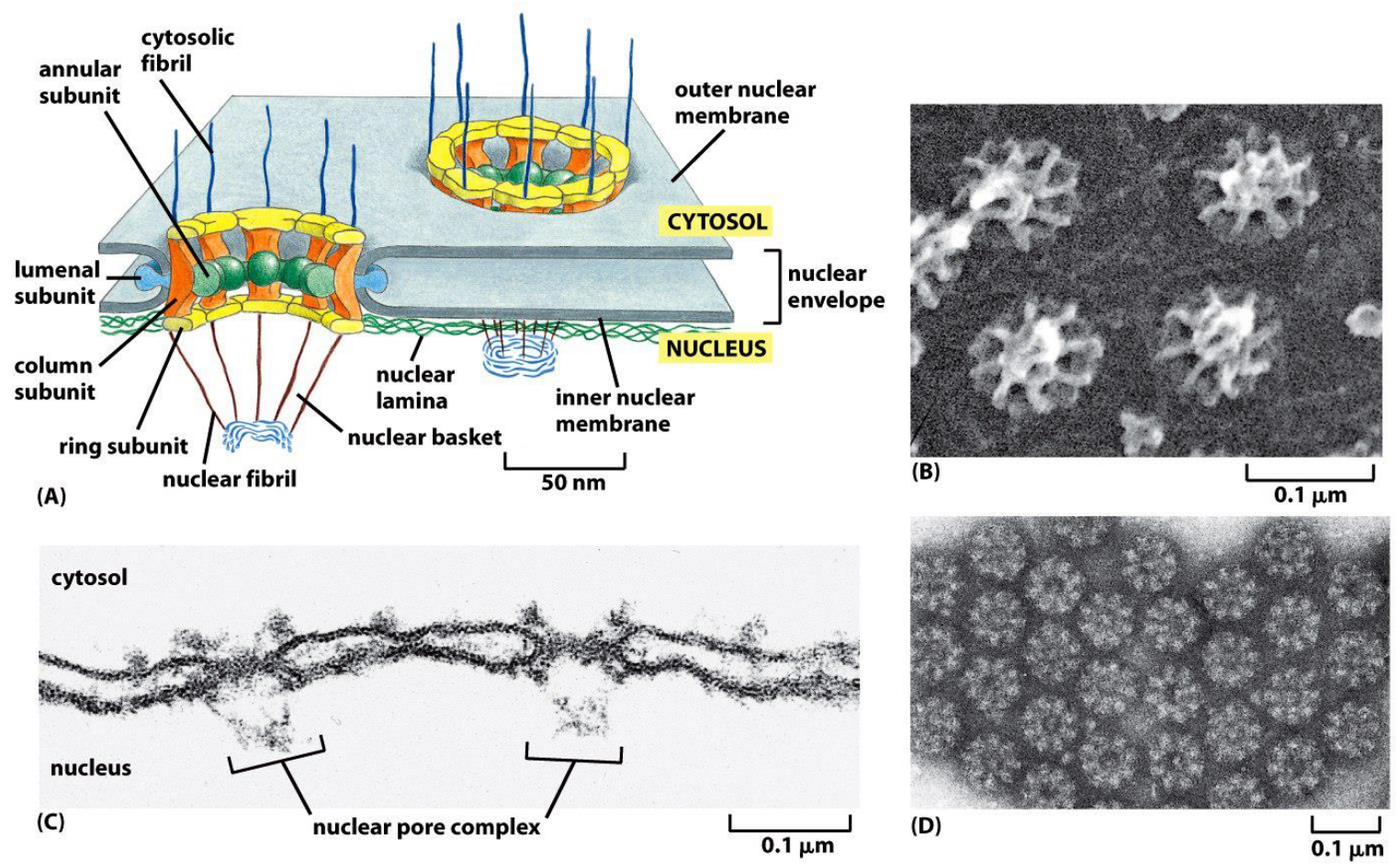

Figure 6-2 Organization of NPCs on NE (Alberts et al., 2007)

(A) Depiction of NPC components. Electron micrograph of (B) NPCs from nuclear side of NE, (C) NPCs from side view, (D) NPCs from cytoplasmic side of NE. 
Nuclear envelope is punctured by thousands of very large protein assemblies called nuclear pore complexes (NPCs), and NPCs are the main routes of transport between the nucleus and the cytoplasm (Figure 6-2). One of the first visible features of NPCs was its eight-fold symmetry (Watson, 1959). The total size of the NPC is estimated to be $66 \mathrm{MDa}$ in yeast (Rout and Blobel, 1993) and 125 MDa in vertebrates (Reichelt et al., 1990). NPCs restrict the diffusion of large proteins, which can be aided by nuclear transport receptors (NTRs) for NPC passage. It was shown that particles up to $39 \mathrm{~nm}$ in diameter can pass through the NPCs (Pante and Kann, 2002; Au and Pante, 2012). These gigantic protein assemblies are made up of only 30 different proteins (Figure 6-3) called nucleoporins (Nups) that exist in different copy numbers (Ori et al., 2013). Structural organization of these proteins is still under debate with many proposed models (for a review of models see: Bilokapic and Schwartz, 2012).

Basically, Nups can be divided into two classes; the structural Nups that make up the ring like scaffold sitting on the NE, and Nups with unstructured regions that fill up the gap in the center of the ring and plug the pore. A more comprehensive depiction of structural elements of each vertebrate Nup can be seen on Figure 6-3 (Schwartz, 2005).

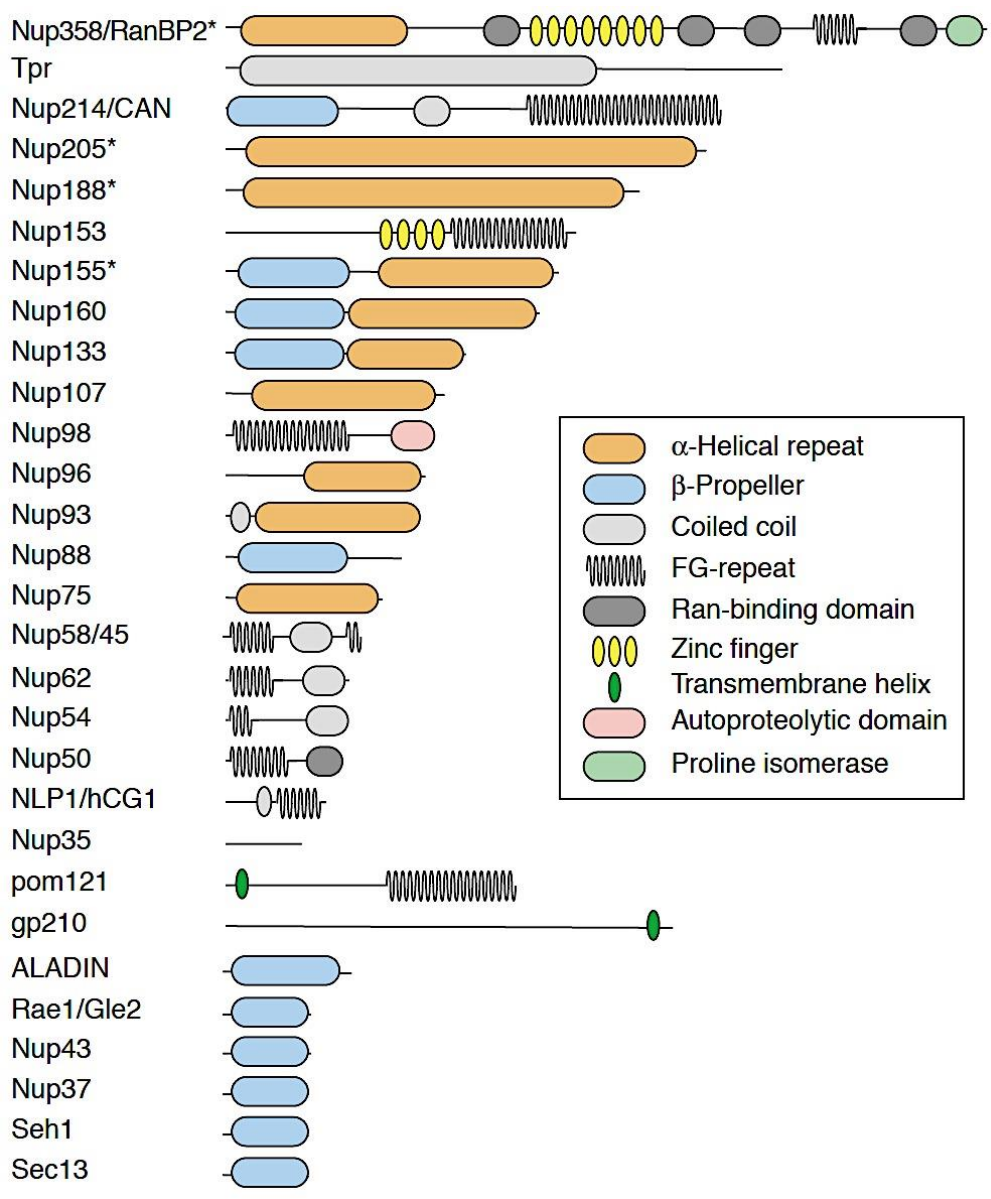

Figure 6-3 Structural elements of vertebrate nucleoporins (Schwartz, 2005)

${ }^{*} \alpha$-Helical regions predicted with high certainty 
The second group is composed of proteins with dispersed phenylalanine-glycine repeats (FG repeats), which lacks a definite structure (Denning et al., 2003). FG repeats are the sites of interaction with NTRs (Iovine et al., 1995; Radu et al., 1995; Rexach and Blobel, 1995; Bayliss et al., 1999, 2000). Some FG repeat regions show self-interaction, and they can form hydrogels in vitro. FG hydrogels can reproduce the two essential functions of NPCs; blocking passively diffusing cargoes (inert cargoes) and enriching NTR. cargo complexes (Frey et al., 2006; Frey and Gorlich, 2007, 2009; Labokha et al., 2013). This observation is in line with the previously suggested "selective phase model" which suggests the NPC permeability barrier being a hydrogel made of a meshwork of FG domains (Ribbeck and Gorlich, 2001).

\subsection{TRANSPORT THROUGH THE NPCS}

NPCs are the main routes of macromolecule exchange between the nucleus and the cytoplasm. Cells invest quite some resources to maintain this exchange with many dedicated proteins that also involve abundant ones. Transport through the NPC differs from protein import into mitochondria, chloroplast or endoplasmic reticulum since proteins are transported through the NPC in a folded form and sometimes as complexes of different macromolecules.

NPCs efficiently block passive diffusion of inert molecules with a diameter $\geq 5 \mathrm{~nm}$ (Mohr et al., 2009). While a small macromolecule like ubiquitin can pass through the NPC freely, a larger macromolecule would need the aid of nuclear transport receptors (NTRs) for efficient transport. Not only size but also charge may contributes to selectivity. Positively charged proteins were suggested to be excluded better than negatively charged proteins due to the positive net charge of the NPC channel proteins (Colwell et al., 2010). The impressive examples of NTR cargoes with large size include ribosomal subunits, Balbiani ring particles, and intact viral capsids (Stevens and Swift, 1966; Franke and Scheer, 1974; Whittaker and Helenius, 1998; Au and Pante, 2012).

\subsubsection{Passive Diffusion Through the NPCs}

Passive diffusion of molecules between the cytoplasm and the nucleus proceeds through either the NE or the NPCs. Small organic substances like steroids, glycerol or ethanol can pass through the double lipid bilayer. As suggested by the "selective phase model", the FG meshwork in NPC possesses an aqueous passive diffusion barrier (Ribbeck and Gorlich, 2001). Passive diffusion through NPCs is fast for small molecules and does not require a special interaction with the NPC components. As the size gets larger passive diffusion becomes limiting for the efficient translocation; spherical proteins larger that $20-30 \mathrm{kDa}$ ( $\geq 5 \mathrm{~nm}$ in diameter) are already delayed for NPC passage (Paine et al., 1975; Bonner, 1975; Mohr et al., 2009). 


\subsubsection{Facilitated Active Transport}

Macromolecules that cannot overcome the NPC barrier due to a large size or other features like charge are transported in a facilitated manner. Nuclear transport receptors (NTRs) are large molecules (90-150 kDa) that are able to shuttle between nucleus and cytoplasm. NTRs bind to cargoes and help them go through the barrier. Most NTRs are members of the Importin $\beta(\operatorname{Imp} \beta)$ superfamily. Their multivalent interactions with the FG meshwork allow them to enter the NPC barrier very efficiently. Most NTRs carry cargoes in one direction. They are called importins when they import cargoes from the cytoplasm to the nucleus, and exportins if exporting cargoes from the nucleus to the cytoplasm (Gorlich et al., 1994; Fornerod et al., 1997). Some NTRs, like Exportin 4, can function in both ways with different cargoes (Gontan et al., 2009). NTR aided transfer is so efficient that up to 1000 translocations can take place in a single NPC per second (Ribbeck and Gorlich, 2001).

\subsection{DIRECTIONALITY OF THE TRANSPORT}

NTRs can shuttle between the cytoplasm and the nucleus, and can bind to their cargos, but these are not enough for a directional transport. For binding to its cargo in a compartment and releasing it in the other one, NTRs require means of sensing the location. All Imp- $\beta$ like NTRs bind to a small guanine nucleotide binding protein called Ran (Gorlich et al., 1997; Fornerod et al., 1997). Ran stands for Ras-related nuclear protein and is a 25 kDa GTPase (Drivas et al., 1990; Bischoff and Ponstingl, 1991; Melchior et al., 1993a). The GTPase function enables Ran to switch between two states; the GTP bound active state (RanGTP) and the GDP bound silent state (RanGDP). RanGTP is the active state because it binds to Imp $\beta$-like NTRs while RanGDP does not. Nucleus and cytoplasm differ in their RanGTP concentration; the nucleus has 1000 fold RanGTP concentration than the cytoplasm (Gorlich et al., 2003). This steep RanGTP gradient acts as the fuel of the directional transport (Gorlich et al., 1996).

Exportins bind to their cargoes in the nucleus and assemble into export complexes with RanGTP, and in the cytoplasm, export complex is disassembled by involvement of other factors (explained below). Free exportin does not re-bind to its cargo, but returns to the nucleus and is ready for another round of transport. The export complex is formed by cooperative interaction, if one of the binders is present (RanGTP or cargo), affinity for the second one is increased, and upon binding complex is stabilized (Kutay et al., 1997; Petosa et al., 2004; Monecke et al., 2013).

On the other hand, importins form complexes with their cargoes in the cytoplasm where RanGTP levels are very low. When import complex passes through the NPC, it is disassembled upon RanGTP binding to importin in the nucleus. RanGTP binding is strong and incompatible with cargo 
binding, and prevents importin-cargo interaction. The importin. RanGTP complex returns to the cytoplasm and after dissociation of RanGTP, it is ready for the next cargo. Transport of NTRs alone or as complexes through the FG meshwork is reversible and does not require energy (Kose et al., 1997; Nakielny and Dreyfuss, 1998; Schwoebel et al., 1998; Ribbeck et al., 1999; Englmeier et al., 1999; Nachury and Weis, 1999; Zeitler and Weis, 2004).

Both for import and export cycles there is a net flux of RanGTP from the nucleus to the cytoplasm. Cells employ a transport receptor called nuclear transport factor 2 (NTF2) to efficiently carry RanGDP from the cytoplasm back to the nucleus (Ribbeck et al., 1998). NTF2 is not an Imp $\beta$-like NTR by the structure definition (Bullock et al., 1996). It is a $15 \mathrm{kDa}$ protein that is found as homodimer in the cell. The dimer can bind to two RanGDPs. NTF2 cargo release is linked to the conversion of RanGDP to RanGTP as it enters to the nucleus. This transport cycles are summarized in Figure 6-4. 


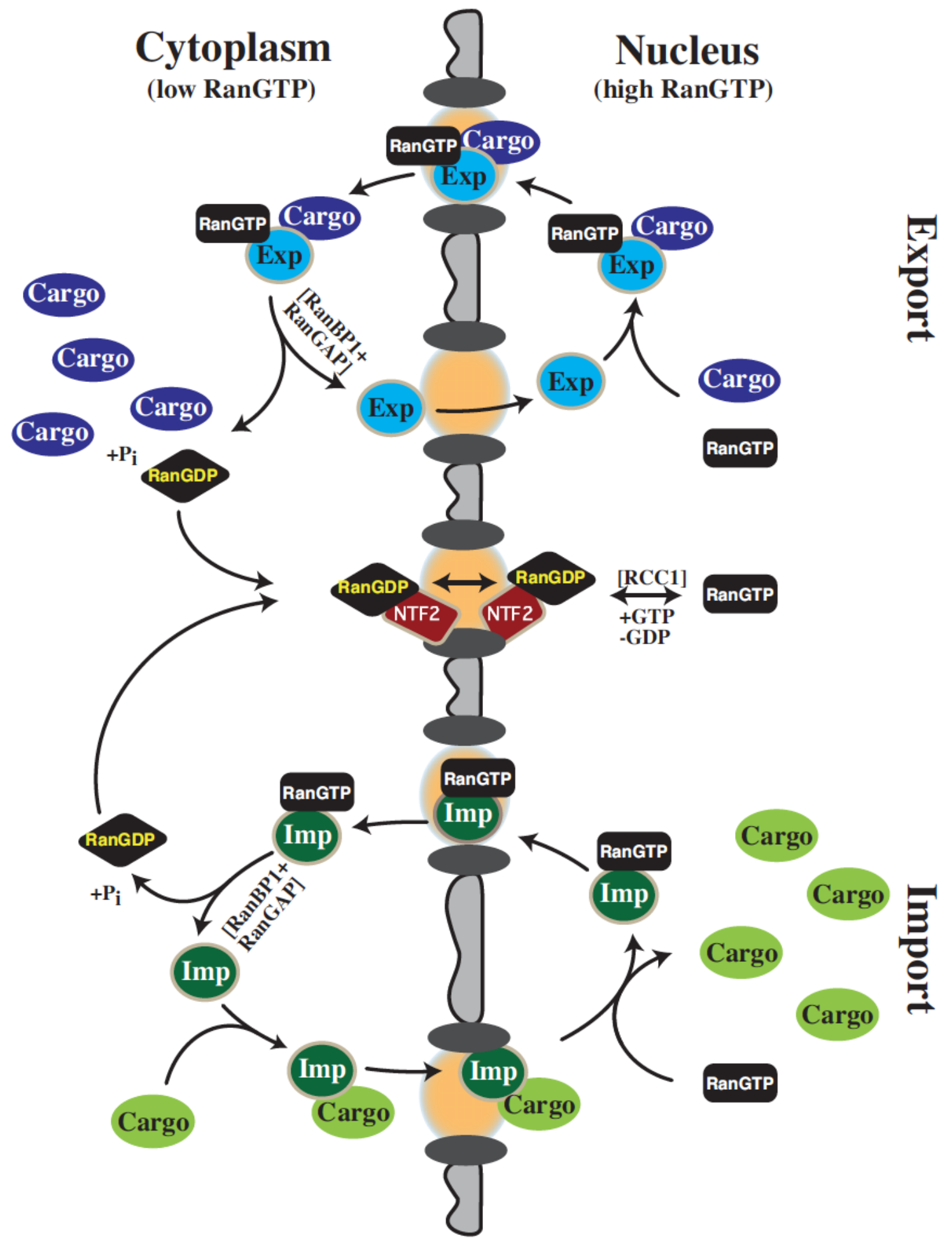

Figure 6-4 Overview of active nucleocytoplasmic transport through NPCs modified from (Gorlich and Kutay, 1999)

This elegant RanGTP gradient has other players on the backstage. Although Ran is a GTPase, it has a very low intrinsic activity. For an efficient hydrolysis, RanGTP needs stimulation of its GTPase activating protein RanGAP. RanGAP can increase the GTPase activity of Ran by $10^{5}$ fold (Bischoff et 
al., 1994). Human RanGAP, RanGAP1, has a modular organization; an N-terminal leucine rich repeat (LRR) region and a C-terminal domain that gets sumoylated.

The N-terminal LRR domain has the GTPase activating activity, and the C-terminal domain gets sumoylated by Ubc9 and triggers RanGAP interaction with cytoplasmic side of NPCs via Nup358 (Mahajan et al., 1997) (Gareau et al., 2012). While human RanGAP1 is localized to the NPCs, yeast and S.pombe orthologs Rna1p lack the C-terminal domain and it is localized to the cytoplasm. In all homologs, LRR domain is followed by a poly glutamic acid region (Figure 6-5) (Hopper et al., 1990) (Melchior et al., 1993b).
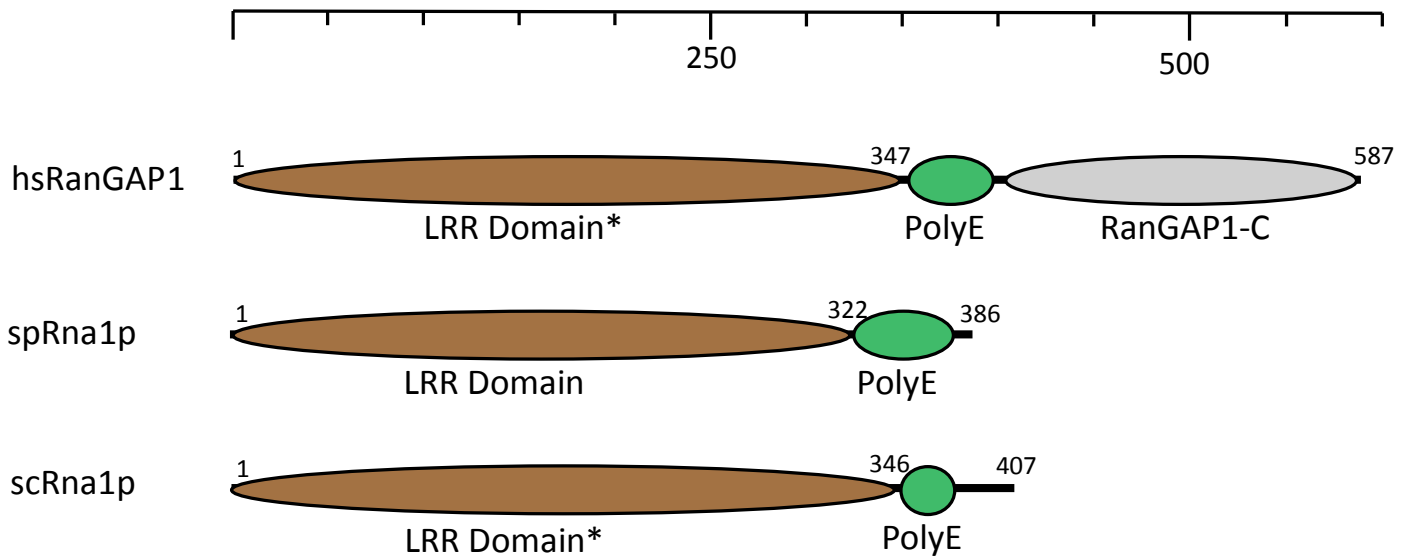

Figure 6-5 Domain organization of RanGAP homologs

Domains assigned by homology are indicated with '*'. LRR stands for leucine rich repeat domain and responsible for GTPase activation, PolyE represents the poly glutamic acid region. Domains are drawn to the scale (50 amino acids). End of LRR domain and protein are also indicated on the domain representations.

RanGAP can act on RanGTP, but RanGTP in export complexes is not accessible for RanGAP since the binding surface on Ran is overlapping for RanGAP and NTRs (Paraskeva et al., 1999) (Seewald et al., 2002) (Monecke et al., 2009). It requires the assistance of RanBP1 or RanBP2/Nup358 which bind to the C-terminal loop on RanGTP in the export complex and destabilize it. (Yokoyama et al., 1995; Bischoff and Gorlich, 1997; Koyama and Matsuura, 2010). When RanGAP and RanBP1 bind RanGTP, its GTPase activity is stimulated $\sim 10^{6}$ fold; RanBP1 contributes about 10 fold to the activation by RanGAP (Bischoff et al., 1995).

Conversion of RanGDP to RanGTP is stimulated by the nuclear protein Ran guanine nucleotide exchange factor (RanGEF), also called RCC1 (regulator of chromosome condensation 1 ). RCC1 acts specifically only on Ran and stimulates the exchange of nucleotide (Bischoff and Ponstingl, 1991). RCC1 interaction does not have any preference towards GTP or GDP bound Ran, but the high 
molar ratio of GTP to GDP in the cell drives RanGDP conversion to RanGTP (Bischoff and Ponstingl, 1991).

RanGAP, RanBP1 and RanBP2 take role in stimulation of GTPase activity of Ran. These proteins are kept cytoplasmic, or on the cytoplasmic side of the NPC (Hopper et al., 1990; Yokoyama et al., 1995; Richards et al., 1996; Matunis et al., 1996; Mahajan et al., 1997; Saitoh et al., 1997). On the other hand, RCC1 is chromatin bound, and constraints RanGTP generation to nucleus (Ohtsubo et al., 1989).

Ran can act as a switch, because it undergoes drastic conformational changes in more than one position upon GTP hydrolysis. The core is mostly stable, but 3 regions show rearrangement upon GTP hydrolysis; switch-I (residues 30 to 47), switch-II (residues 65 to 80), and C terminal switch-III (residues 177 to 216)(Figure 6-6). In RanGTP structure, $\mathrm{a} \mathrm{Mg}^{+2}$ ion and hydrogen bonds coordinate $\beta$ and $\gamma$-phosphates of the GTP. Conformational change is triggered by the hydrolysis of the phosphodiester bond, and thus rearrangements in the network of hydrogen bonds. Switch-I is relocated completely and gains a $\alpha$-helical structure. Switch-II undergoes a smaller conformational change than switch-I, but this change is significant since it is in close proximity of the nucleotide. The $\mathrm{C}$ terminal switch-III is the part that shows the most extreme change in the structure. $\mathrm{C}$ terminal switch-III is a long linker followed by a $\alpha$-helical extension and the acidic stretch "DEDDDL". In RanGDP structure the C terminal switch-III is folded back on the globular Ran core and it is in contact with switch-I. Although the acidic stretch is missing from the crystal structure, it most probably contacts the basic patch. In RanGTP structure, the changes in switch-I are transmitted to the $\mathrm{C}$ terminal switch-III, and contribute to its displacement from globular Ran core (Milburn et al., 1990; Scheffzek et al., 1995; Vetter et al., 1999b).

NTR. RanGTP structure with Imp $\beta$, Transportin, and CAS also show that RanGTP is in contact with $\mathrm{N}$ termini of these NTRs (Chook and Blobel, 1999; Vetter et al., 1999a; Matsuura and Stewart, 2004). RanGTP interacts through switch II, basic patch, and some other loops, and most of these regions would not be accessible to NTRs in RanGDP conformation due to C terminal switch-III. 

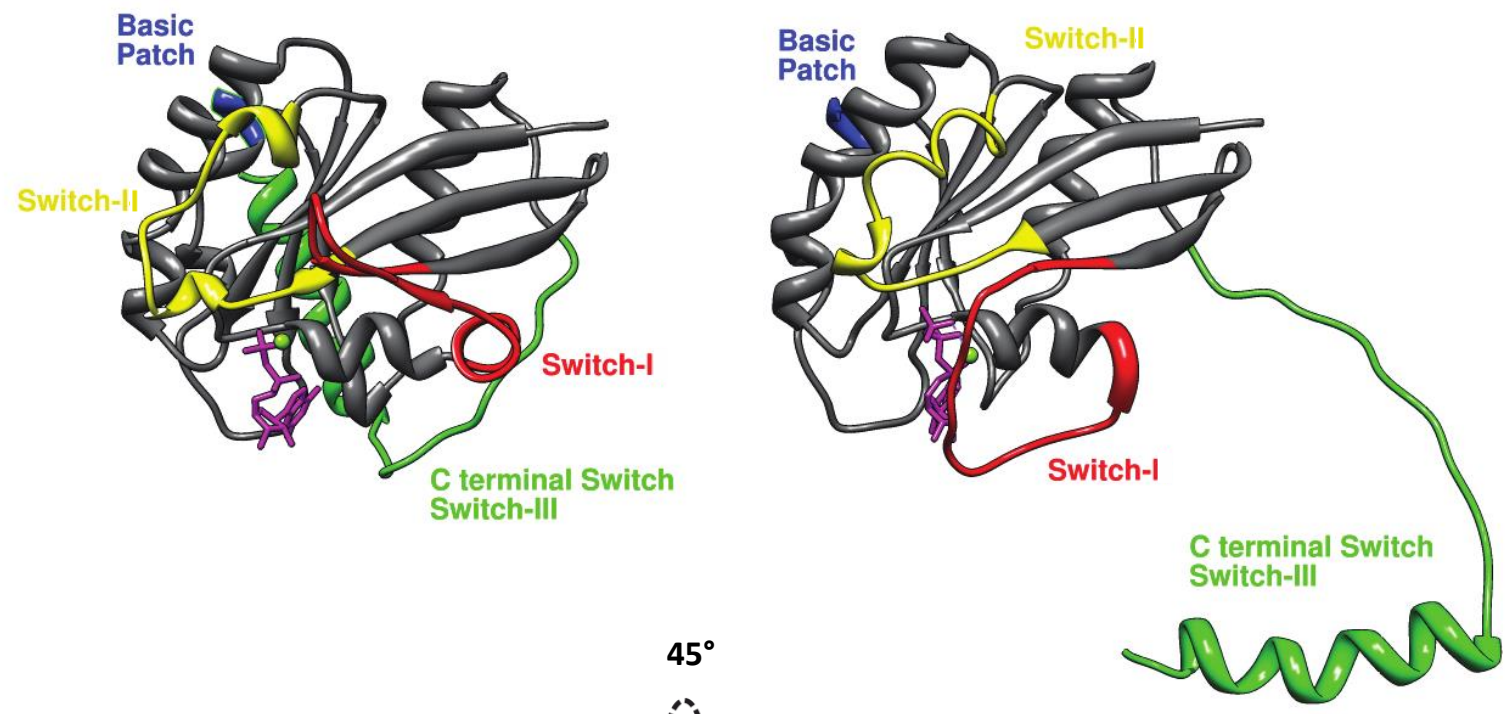

RanGDP

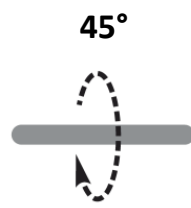

\section{RanGTP}
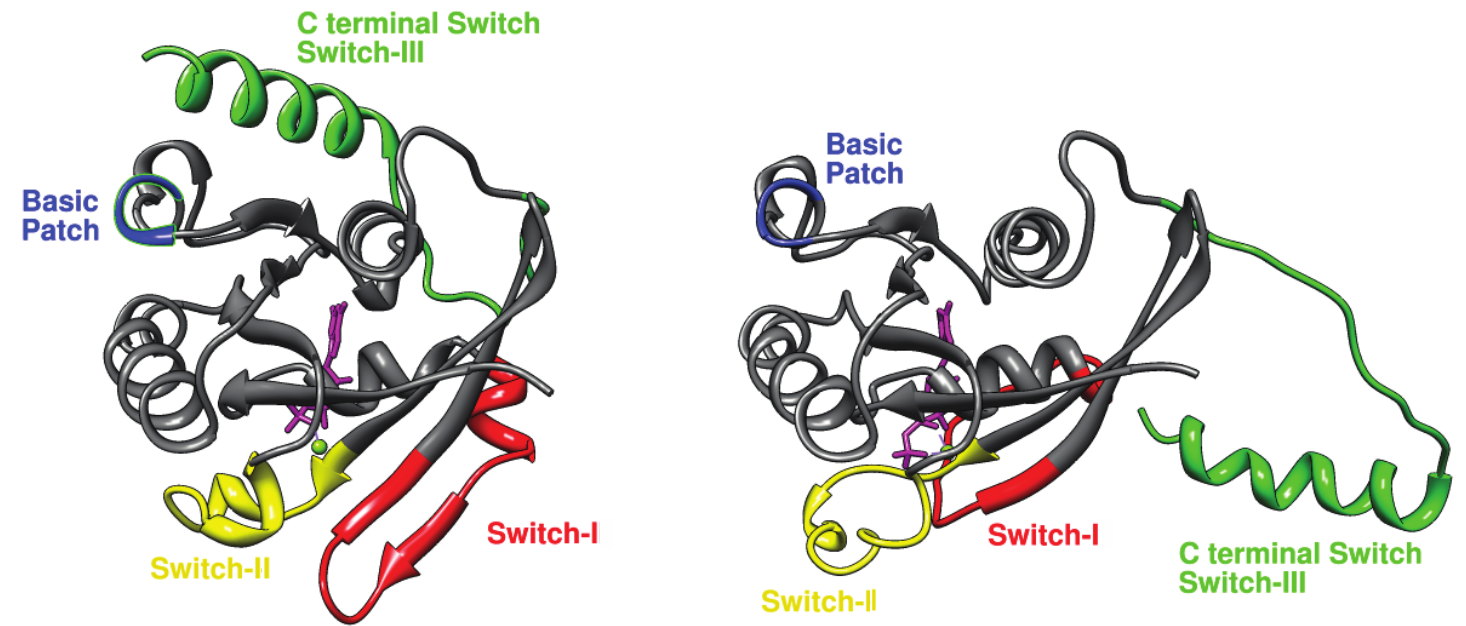

Figure 6-6 Comparison of RanGTP and RanGDP structures

RanGDP structure is from crystal structure with PDB-ID 3GJ0 (Partridge and Schwartz, 2009). RanGTP structure is part of Ran-GPPNHP-RanBD1 crystal structure with PDB-ID 1RRP (Vetter et al., 1999b). From both structures, the overlapping part between amino acids 8 and 207 was visualized with ribbon representation. GDP and GTP were shown as purple sticks. For clarity, RanBP2/RanDB1 domain omitted from RanGTP structure. In both structures some parts of Ran sequence was missing. The overlapping part of two structures; residues 8-207 were used in this representation. Parts of Ran structure that undergo significant changes were indicated on the structure: amino acids 30 to 47 was marked as switch-I (red); 65 to 80 as switch-II (yellow); and 177-207 as C terminal switch-III (green). In the protein sequence, C terminal switch is continued with an acidic stretch, and was missing in the crystal structures. In RanGDP conformation this acidic stretch packs against a basic patch (blue). 


\subsection{NUCLEAR TRANSPORT RECEPTORS}

Imp $\beta$-like NTRs are structurally very similar, they are composed of so-called HEAT repeats (Gorlich et al., 1997), named after the proteins huntingtin, elongation factor 3 , protein phosphatase $2 \underline{A}$, lipid kinase IOR that were the first examples of this structural element (Andrade and Bork, 1995). HEAT repeats are composed of two antiparallel $\alpha$-helices of 10 to 20 amino acids and linked by a short loop. NTR structure is made up of 18-20 HEAT repeats that line up sequentially with an angular shift that gives rise to a right-handed solenoid (Cingolani et al., 1999; Chook and Blobel, 1999; Matsuura and Stewart, 2004; Monecke et al., 2009). Hydrophobic side chains sustain interactions in and between HEAT repeats. Packing is uniform in a way that the first helix of the HEAT repeat faces outside of NTR circle, and the second one faces inside. This organization confers flexibility to the NTRs and helps them to adapt different conformations (cargo bound and non-bound) (Stewart, 2003). Linear arrangement of HEAT repeats also results in a large protein surface that is needed for interaction with RanGTP, respective cargoes and also FG repeats of the NPC. NTRs recognize many different classes of cargos either to import (Table 6-1), or to export (Figure 6-4).

Imp $\beta$ like NTRs share many features. They are made up of the same structural elements, they have acidic isoelectric points ( $\mathrm{pl} 4.0-6.0$ ), yet they have very low overall sequence homology (8 $15 \%)$. The only significant homology is found in the $\mathrm{N}$-terminal region that accounts for interaction with RanGTP (Gorlich et al., 1997).

\begin{tabular}{|c|c|c|}
\hline NTR & Selected Cargoes & References \\
\hline \multirow[t]{4}{*}{ Importin $\beta$ (Imp $\beta-1)$} & Ribosomal Proteins & Gorlich et al., 1995 \\
\hline & HIV Rev, HIV Tat & Huber et al., 1998a \\
\hline & Histones & Jakel and Gorlich, 1998 \\
\hline & Snurportin1.UsnRNPs & Jakel et al., 1999; \\
\hline with Importin 7 & histone $\mathrm{H} 1$ & Truant and Cullen, 1999 \\
\hline with Importin $\alpha$ & Classical NLS-cargoes & Muhlhausser et al., 2001 \\
\hline \multirow{5}{*}{$\begin{array}{l}\text { Transportin 1+2 } \\
\text { (Trn, Imp } \beta-2)\end{array}$} & hnRNP protiens & Pollard et al., 1996 \\
\hline & Ribosomal proteins & Jakel and Gorlich, 1998 \\
\hline & TAP/NFX1 & Truant et al., 1999 \\
\hline & Histones & Muhlhausser et al., 2001 \\
\hline & c-Fos & Arnold et al., 2006 \\
\hline $\begin{array}{l}\text { Transportin SR 1+2 } \\
\text { (TrnSR, Trn 3) }\end{array}$ & SR proteins & Kataoka et al., 1999 \\
\hline \multirow[t]{2}{*}{ Importin 4} & Ribosomal proteins & Mosammaparast et al., 2001 \\
\hline & Histones & Jakel et al., 2002 \\
\hline
\end{tabular}




\begin{tabular}{|c|c|c|}
\hline \multirow[t]{2}{*}{ Importin 5} & Ribosomal Proteins & Jakel and Gorlich, 1998 \\
\hline & Histones & Mosammaparast et al., 2001 \\
\hline \multirow[t]{3}{*}{ Importin 7} & Ribosomal Proteins & Jakel and Gorlich, 1998 \\
\hline & Histones & Muhlhausser et al., 2001 \\
\hline & ERK2, SMAD3, MEK1 & Chuderland et al., 2008 \\
\hline \multirow[t]{2}{*}{ Importin 8} & SRP19 & Dean et al., 2001 \\
\hline & Argonaute proteins & Weinmann et al., 2009 \\
\hline \multirow[t]{2}{*}{ Importin 9} & Ribosomal Proteins & Muhlhausser et al., 2001 \\
\hline & Histones & Jakel et al., 2002 \\
\hline \multirow[t]{2}{*}{ Importin 11} & UbcM2 & Plafker and Macara, 2000b \\
\hline & rpL12 & Plafker and Macara, 2002 \\
\hline \multirow[t]{4}{*}{ Importin 13} & hUBC9, MGN/Y14 & Mingot et al., 2001 \\
\hline & TF NF-Y & Kahle et al., 2005 \\
\hline & CHRAC-15/17 & Walker et al., 2009 \\
\hline & NC2 Complex & Kahle et al., 2009 \\
\hline Exportin 4 & Sox2, SRY & Gontan et al., 2009 \\
\hline
\end{tabular}

Table 6-1 Mammalian importins and selected cargos

\begin{tabular}{|c|c|c|}
\hline NTR & Selected Cargoes & References \\
\hline \multirow[t]{6}{*}{ CRM1 (Exportin 1) } & Leucine rich export signals & Fischer et al., 1995 \\
\hline & HIV Rev.RRE containing RNAs & Fornerod et al., 1997 \\
\hline & Snurportin1 & Paraskeva et al., 1999 \\
\hline & Signal recognition particle & Trotta et al., 2003 \\
\hline & Nmd3.60S Ribosomal Subunit & Alavian et al., 2004 \\
\hline & PHAX.UsnRNAs & Ohno et al., 2000 \\
\hline CAS (Exportin 2) & Importin $\alpha \mathrm{s}$ & Kutay et al., 1997 \\
\hline Exp-t (Exportin 3) & tRNA & Kutay et al., 1998 \\
\hline \multirow[t]{2}{*}{ Exportin 4} & elF5A & Lipowsky et al., 2000 \\
\hline & SMAD3 & Kurisaki et al., 2006 \\
\hline \multirow[t]{3}{*}{ Exportin 5} & aa-tRNA.eEF1A & Bohnsack et al., 2002 \\
\hline & dsRNA.dsRNA binding proteins & Brownawell and Macara, 2002 \\
\hline & pre-miRNAs & Bohnsack et al., 2004 \\
\hline Exportin 6 & Actin.profilin & Stuven et al., 2003 \\
\hline Exportin 7 & p50RhoGAP, 14-3-30 & Mingot et al., 2004 \\
\hline Importin 13 & elF1A & Mingot et al., 2001 \\
\hline
\end{tabular}

Table 6-2 Mammalian exportins and selected cargoes 


\subsubsection{CRM1/Exportin 1}

CRM1 (chromosomal region maintenance 1) was first found in Schizosaccharomyces pombe genetic screen with a cold sensitive mutation that resulted in deformed chromosomes, and was not recognized as a nucleocytoplasmic transport related protein (Adachi and Yanagida, 1989). Later it was found as the target of toxin Leptomycin B (Nishi et al., 1994).

The leucine rich nuclear export signals (NESs) were discovered in HIV-1 Rev protein and protein kinase $\mathrm{A}$ inhibitor (PKI), although it was known that a mediator was involved in the nuclear export of these proteins, the identity of the respective NTR was not clear (Fischer et al., 1995; Izaurralde and Mattaj, 1995; Wen et al., 1995; Gorlich and Mattaj, 1996). Later CRM1 was identified as the nuclear transport receptor of these proteins with NESs (Fornerod et al., 1997; Fukuda et al., 1997; Neville et al., 1997; Ossareh-Nazari et al., 1997). Since then, the library of proteins that are exported by CRM1 via an NES grew tremendously. A curated database of CRM1 cargoes with validations at different experimental settings has more than 250 entries from various species.

\section{Exportint tRNA}

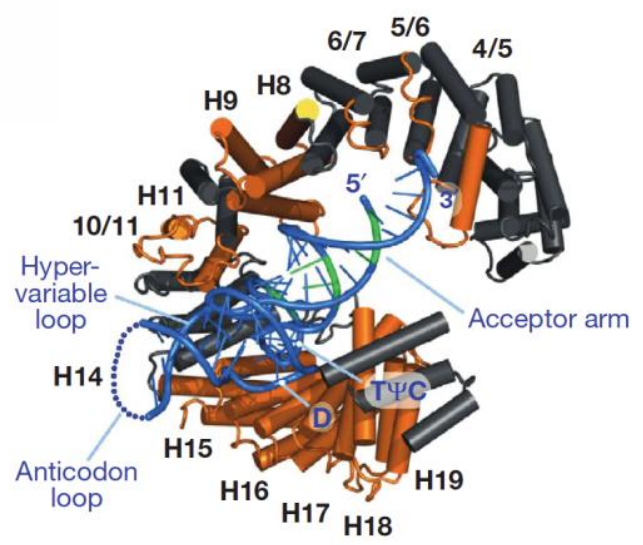

Exportin5 p e-miRNA

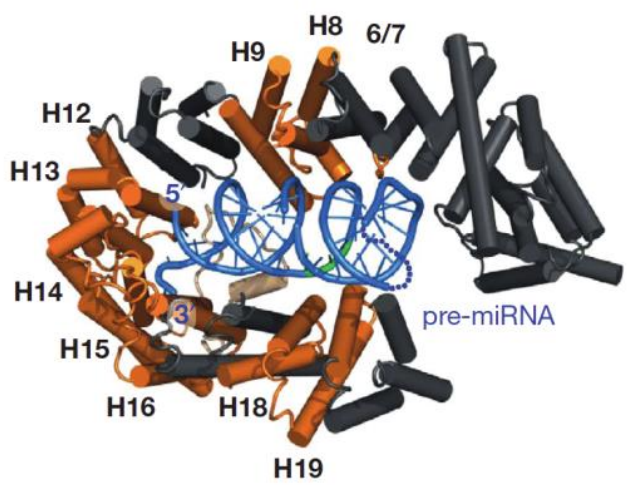

CAS Importina

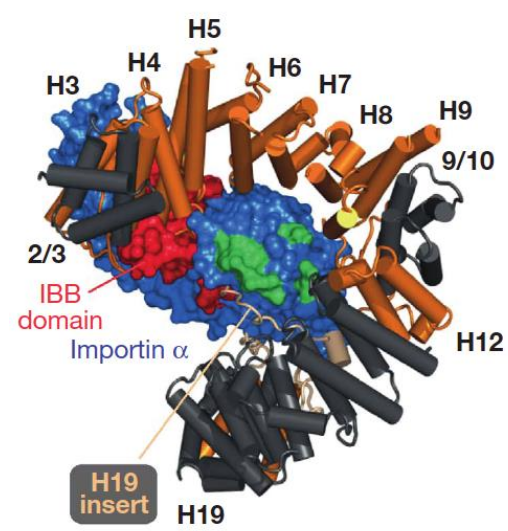

CRM1 Snurportin1

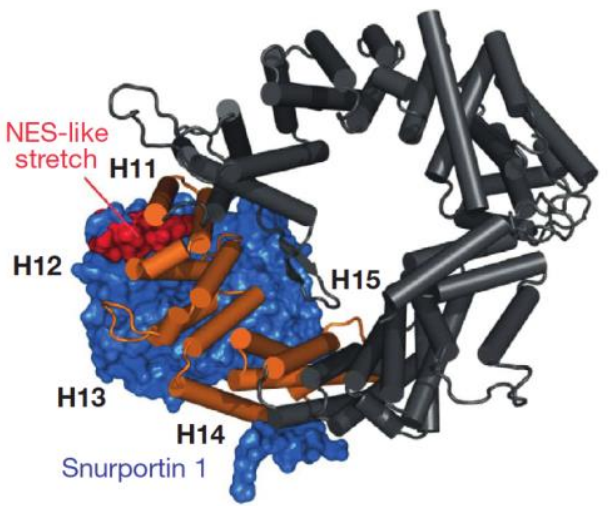

Figure 6-7 Exportin structures with respective cargoes adapted from (Güttler and Görlich, 2011).

Crystal structures of 4 exportins cargo RanGTP complexes are shown without RanGTP. Cargoes are in blue and their contacting helices in NTRs are colored orange. Residues interacting with RanGTP on cargoes are marked green. 
The large number of cargoes nominates CRM1 as the most promiscuous NTR of the cell. It recognizes various cargos that are structurally and functionally distinct. This feature of CRM1 can be attributed to its cargo recognition mechanism that is different from other exportins. Several exportins have been crystalized in complex with RanGTP and the respective cargoes. Comparison of these structures point out that exportins other than CRM1 wrap their cargos with the inner surface of the solenoid NTR structure, while cargo binding of CRM1 is on its outer surface with a limited interaction area (Cargo interaction surfaces of NTRs are colored orange in Figure 6-7)(Güttler and Görlich, 2011). One should note that the interaction surface of CRM1 with Snurportin 1 is far larger than the interaction surface with the NES only.

Snurportin 1. CRM1 structure was the first crystalized CRM1 cargo complex, due to its high stability. This is sustained by interaction surfaces in addition to the N-terminal NES (Monecke et al., 2009). CRM1 interacts with Snurportin 1 tighter than with its other export substrates, because CRM1 is not only the export factor of Snurportin 1, but also acts as the disassembly factor for imported Snurportin 1. U snRNP complex (Huber et al., 1998b). The exported Snurportin 1 would be ready for another cycle of U snRNP import. CRM1 interactions with other cargoes are less stable and might have even smaller interaction surface with CRM1. The interaction surface of CRM1 with NESs is a hydrophobic cleft build by 4 neighboring $\alpha$-helices.

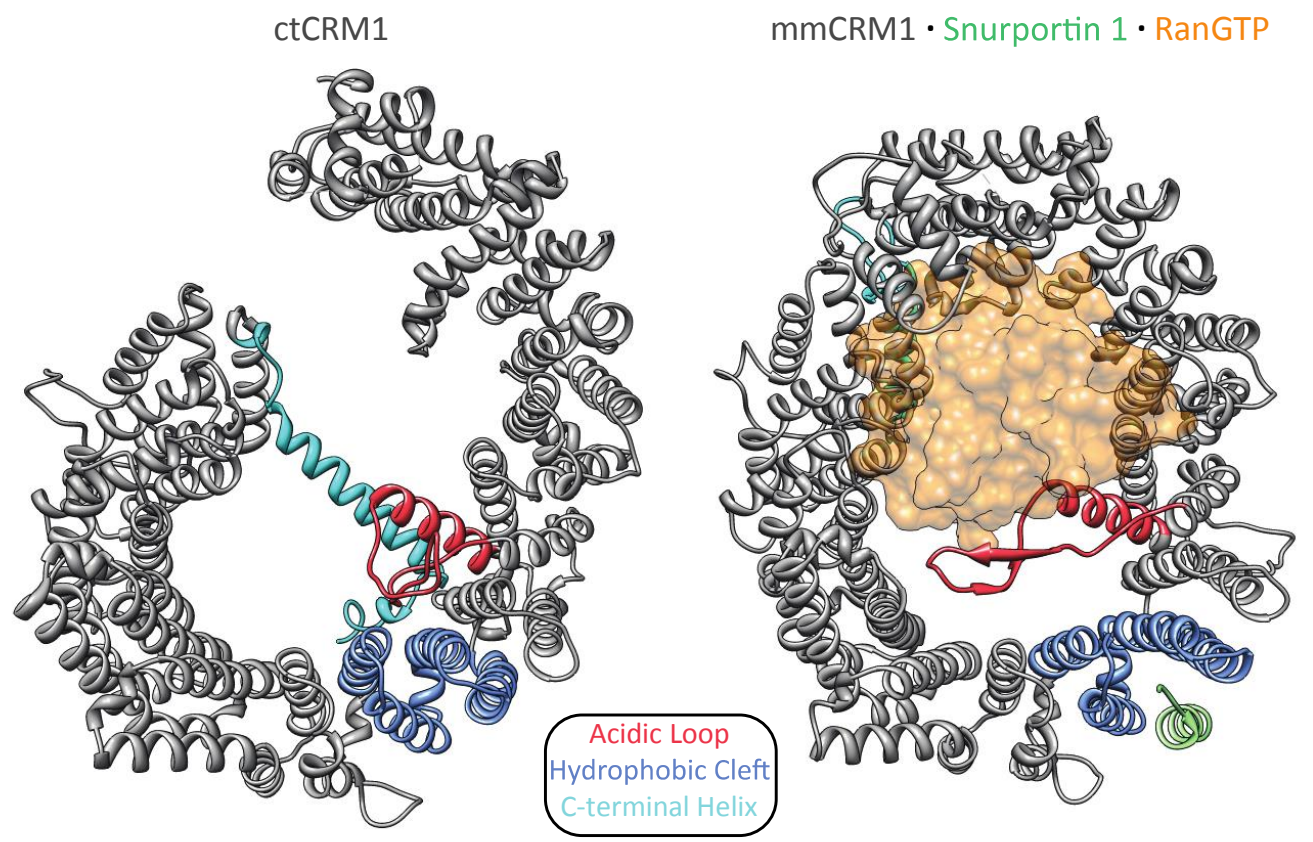

Figure 6-8 Free and cargo bound states of CRM1

Structural comparison of free CRM1 structure from Chaetomium thermophilum (PDB ID 4FGV), and human Snurportin 1 and RanGTP bound mmCRM1 structure (PDB ID 3GJX). RanGTP was represented as orange transparent surface, and Snurportin 1 NES was represented as green ribbon. For clarity, rest of the Snurportin 1 structure was omitted. 3 regions that show great flexibility and important for the stabilization of different states were colored. Acidic loop (ctCRM1 ${ }^{421-460}$,

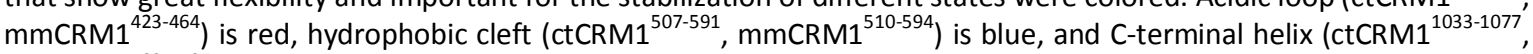
$\mathrm{mmCRM1} 1^{1021-1071}$ ) is cyan. 
Comparison of exportin structures also reveals another aspect of CRM1 export mechanism. In the cases of exportins CAS, Exportin-t and Exportin 5, cargoes interact not only with their respective NTRs but also with RanGTP. On the other hand, CRM1 serves as a platform that RanGTP and Snurportin 1 bind on separate surfaces. RanGTP and Snurportin 1 are not in direct contact. Although these interactions are spatially separated, they favor the same structural conformation of CRM1, and bind to CRM1 in a cooperative manner (Monecke et al., 2009).

CRM1 has two different conformations, the relaxed conformation that is free of a cargo and RanGTP, and the strained conformation that is stabilized by RanGTP and NES binding (Dong et al., 2009; Monecke et al., 2009; Dian et al., 2013; Monecke et al., 2013). The main functional difference between the two conformations is the opening of the hydrophobic cleft on CRM1. The distance between the helices $11 \mathrm{~A}$ and $12 \mathrm{~A}$ are considerably different, and in the stained conformation they are separated enough to accommodate an NES in between. This strained conformation is stabilized by RanGTP binding with two mechanisms. First, the $\mathrm{C}$ terminal helix that stabilizes the relaxed conformation is displaced, and N and C-terminus of CRM1 are brought together. Second, the acidic loop is reorganized to form a $\beta$ hairpin. In RanGTP bound conformation, the acidic loop extends towards the center of CRM1 and reaches to the other side of the toroid structure and touches helix 15B (Figure 6-8).

High RanGTP concentration in the nucleus drives CRM1 to strained conformation that is ready to accept the incoming NESs, and binding of NES further stabilizes this conformation. Upon arrival to the cytoplasm, RanBP1 or RanBP2 binding to RanGTP disassembles the export complex and RanGAP converts RanGTP to RanGDP. With low levels of RanGTP in the cytoplasm, cargo is not able to bind to CRM1. CRM1 goes back to the nucleus and performs another cycle of export. 


\subsection{NUCLEAR EXPORT SIGNALS}

Nucleocytoplasmic transport is made possible by a reversible binding of cargo to its respective NTR under regulation of RanGTP. The toroid shape of importin $\beta$ like transport receptors have large protein surface to fulfill this function. NTRs interact with FG repeat proteins of the NPC with their outer surface. RanGTP interacts with the N-terminal B helices, and sits in the inner gap of the toroid. For most NTRs, the inner surface of this toroid is also the binding platform for the transported cargoes. CRM1 is an exception where NES binding hydrophobic cleft is on the outer surface. The large inner surface of NTRs confers many possibilities for interaction with respective cargoes. A nuclear export signal (NES) is a short amino acid stretch that directs proteins to the cytoplasm utilizing the essential NTR CRM1. Investigation of NESs revealed many aspects of NESmediated transport.

Not all NES containing cargoes are constitutively exported from the nucleus, meaning that CRM1mediated export can be a regulated transport. Many ways of NES-dependent export regulation have been suggested. Regulated accessibility of NESs (Li et al., 1998; Stommel et al., 1999a; Seimiya et al., 2000; Heerklotz et al., 2001; Kobayashi et al., 2001; Craig et al., 2002), phosphorylation (Engel et al., 1998; Ohno et al., 2000; McKinsey et al., 2001; Zhang and Xiong, 2001; Brunet et al., 2002) and also by oxidation, e.g., disulfide bond formation (Yan et al., 1998; Kudo et al., 1999b; Kuge et al., 2001).

The concept of an NES was first suggested relying on the observations that some proteins continuously shuttle between the cytoplasm and the nucleus (Wen et al., 1994; Fischer et al., 1995; Gerace, 1995). These proteins included hnRNP A1 (Pinol-Roma and Dreyfuss, 1992), HIV-1 Rev protein (Kalland et al., 1994; Meyer and Malim, 1994), cAMP-dependent protein kinase (PKA) (Harootunian et al., 1993; Fantozzi et al., 1994), some transcription factors (Madan and DeFranco, 1993) and hsc70 (Mandell and Feldherr, 1990), and they had the potential to bear an NES besides a nuclear localization signal (NLS). A thorough analysis of two of these proteins, Rev and PKA revealed the first NESs.

Inactive PKA holoenzyme consists of two regulatory and two catalytic subunits, and is localized to the cytoplasm. Binding of CAMP to the regulatory subunits triggers the dissociation of the monomeric catalytic subunits, which then can diffuse into the nucleus. Activity of catalytic subunit is strictly regulated and is inactivated by binding of 74 amino acids long protein kinase inhibitor (PKI). Binding of PKI not only inhibits the enzymatic function but also leads to nuclear exclusion of the catalytic subunit (Fantozzi et al., 1994). The sequence that was responsible for nuclear exclusion was a 10 amino acids stretch on PKI. Fusion of fluorescently labeled proteins to this 
fragment restricted their localization to the cytoplasm, and identified it as the first NES (Wen et al., 1994).

Rev is an essential protein for virus reproduction (Cullen, 1992). It takes role in export of viral RNA from the nucleus to the cytoplasm. Two important sequence elements were discovered on Rev, an RNA stem loop interaction motif called Rev response element (RRE), and a C-terminal leucine rich activation domain. This 10 amino acid long leucine rich activation domain was enough to direct other conjugation partners like BSA to the cytoplasm and identified as the second NES (Fischer et al., 1995).

Later CRM1 was identified as the NTR responsible for the transport of NES harboring cargoes (Fornerod et al., 1997; Fukuda et al., 1997; Neville et al., 1997; Ossareh-Nazari et al., 1997). After the identification of the first NES examples, the library of NES containing CRM1 cargoes grew rapidly. Different groups compiled curated NES libraries, or constructed mutant NES libraries, and by analyzing them, they tried to come up with consensus definitions to predict NES sequences.

The first attempt was done by randomization of Rex activation domain. Rex is the functional equivalent of Rev in T-cell leukemia virus type 1, and it also has an NES termed activation domain. By randomization of the activation domain a library was constructed. Then this library was tested for functionality of the activation domain. By aligning the functional sequences, the prominent residues and their spacing was combined in to the consensus $L-X_{2,3}$-[FILVM]- $\mathrm{X}_{2,3}-\mathrm{L}-\mathrm{X}-[\mathrm{LI}]$, and this definition led to the term leucine rich nuclear export signal (IrNES) (Gerace, 1995; Bogerd et al., 1996).

la Cour et al. compiled the first curated database of NES containing proteins in NESbase 1.0. This database contains 80 NES sequences on 75 proteins (la Cour et al, 2003). Only 25 of these NES were defined by the previous IrNES consensus. This database was later used for construction of the first NES prediction algorithm NetNES. Two training sets were generated from the validated NES sequences and NES containing protein sequences excluding NESs. These two sets were used to train a machine-learning algorithm. The allowed hydrophobic residues were increased to L, I, $\mathrm{M}, \mathrm{V}$ and $\mathrm{F}$ at 4 positions, and $\mathrm{E}, \mathrm{D}$ and $\mathrm{S}$ residues were preferred as spacers. This new consensus, [FILVM]-X,3 ${ }_{2,3}$ [FILVM]- $\mathrm{X}_{2,3}$-[FILVM]-X-[FILVM] was able to cover 50 of the 75 NESs in the database (la Cour et al., 2004).

A third study was based on a screen of random peptides for their exclusion from the nucleus. This study found 101 different peptides that were export competent, and grouped them into three different classes. Hydrophobic positions were termed as $\Phi$ positions. L, I, M, V and F were allowed 
at $\Phi$ positions, and $C, W, A$ and T were also allowed only at one $\Phi$ position. Proline residues in the spacer residues were enough to prevent the export, so proline was excluded from the spacer residues. The class I consensus $\Phi-X_{2,3}-\Phi-X_{2,3}-\Phi-X_{2}-\Phi$ was the same as the previous consensus, and covered 83 of the 101 functional NESs. Class II consensus $\Phi-X-\Phi-X_{2}-\Phi-X-\Phi$ and Class III consensus $\Phi-X_{2,3}-\Phi-X_{2,3}-\Phi-X_{2}-\Phi$ were novel and rare, and together they covered 17 of 101 functional NESs. Although these three classes can explain 99 out of 101 artificial NESs, they can only cover 89 of 159 naturally occurring NESs (Kosugi et al., 2008). These definitions were not available as an NES prediction tool.

A second computational approach after NetNES came from Fu et al.. They also constructed two data sets of true and false NESs of 60 proteins selected from NESbase 1.0. Consensus was defined with three $\Phi$ positions, $\Phi-X_{2,3}-\Phi-X-\Phi$, and $\Phi$ positions were limited to $L, I, V, M$ and $F$. The sequences were analyzed for various parameters these parameters were evaluated by LIBSVM (Chang and Lin, 2001) to find the features that gave the significant differences between true and false NESs. These included negative charges in the inter $\Phi$ positions and disorder tendencies, and used in the prediction algorithm NESsential (Fu et al., 2011).

The latest curated library of CRM1 cargoes was compiled by Xu et al. in NESdb. This database contains 221 NES containing cargoes from various species (Xu et al., 2012a). Analysis of these NESs were summarized in 3 consensus sequences, $\Phi-X_{1,2,3}-\Phi-[\wedge W]_{2}-\Phi-[\wedge W]-\Phi$ (type 1 ), $\Phi-X_{2,3}-\Phi-$ $\left[{ }^{\wedge} W\right]_{3}-\Phi-[\wedge W]-\Phi$ (type 2), and $\Phi-X_{2}-\Phi-X[\wedge W]_{2}-\Phi-[\wedge W]_{2}-\Phi$ (type 3), where $\left[{ }^{\wedge} W\right]$ is any of the 20 amino acids except Trp. $\Phi$ positions are either $\mathrm{L}, \mathrm{I}, \mathrm{V}, \mathrm{F}$ or $\mathrm{M}$, and $\mathrm{A}$ and $\mathrm{T}$ residues are allowed only once at either first or second Ф position (Xu et al., 2012b). 
a PKI-class NESs

$\mathrm{X}$ Acidic residues preferred

( $\Phi^{0} \quad \mathrm{I} \approx \mathrm{V} \approx \mathrm{M}>\mathrm{L}>\mathrm{A} \approx \mathrm{Y}>\mathrm{F} \approx \mathrm{W}>\mathrm{P}$

$\mathrm{X}_{2} \quad$ Acidic residues preferred

(1) $\mathrm{L}>\mathrm{I}>\mathrm{V} \approx \mathrm{M}>\mathrm{F}>\mathrm{A}>\mathrm{W}$

$\mathrm{x}_{3}$

$\mathrm{F} \approx \mathrm{M}>\mathrm{L}>\mathrm{I} \approx \mathrm{V}>\mathrm{Y}>\mathrm{W}$

$x_{2}>x_{3}$

( ${ }^{3} \mathrm{~L} \approx \mathrm{M}>\mathrm{I}>\mathrm{V}>\mathrm{F}>\mathrm{W} \approx \mathrm{A}$

$\mathrm{X}$

$\Phi^{4} \quad \mathrm{~L}>\mathrm{I}>\mathrm{M}>\mathrm{V}>\mathrm{F}$

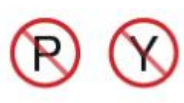

(B)

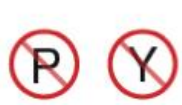

(P) (A) b Rev-class NESs

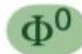

-

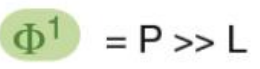

$\mathrm{X}$

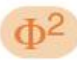

$x_{2}$

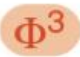

$\mathrm{X}$

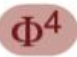

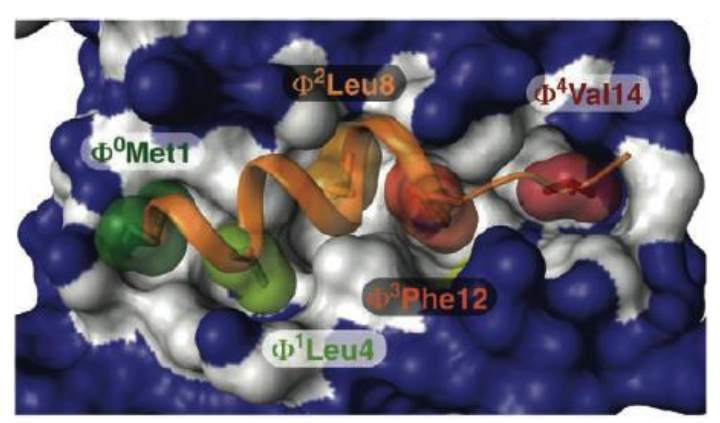

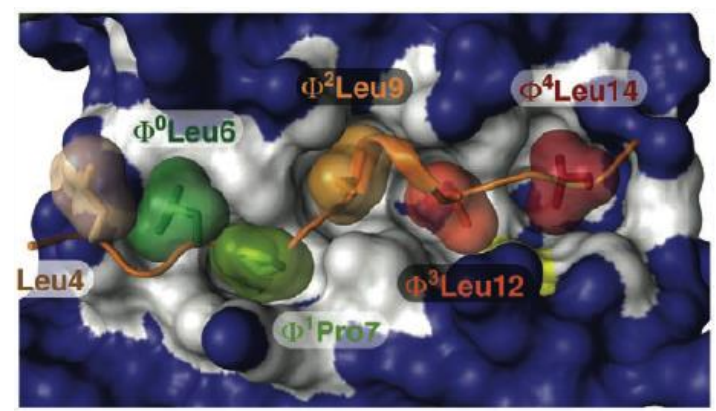

Figure 6-9 Structural definition of NES consensus - modified from (Güttler et al., 2010)

Crystal structures of CRM1 with Snurportin 1 elucidated the true nature of the $\mathrm{N}$-terminal Snurportin 1 NES interaction with CRM1 with 5 Ф positions (Güttler et al., 2010). Güttler et al. replaced the NES with PKI and Rev NESs and obtained two additional crystal structures. PKI NES and Snurportin 1 NES fit into CRM1 hydrophobic cleft with very similar structural orientation, whereas Rev NES is placed in a very different way. This is why these two different NES were separated into PKI type or Rev type NES consensus. Mutation screen of PKI NES $\Phi$ positions also revealed preference of $\Phi$ positions for different amino acids (Figure 6-9). These new definitions of NESs based on the crystal structures were the basis of the new NES prediction tool that I discuss further in the results and discussion. 


\section{RESULTS}

The recently determined crystal structures of CRM1 with bound NESs uncovered some essential details as to how this nuclear export receptor can bind to its various cargoes from a broad range of structural and functional groups. It not only provided an understanding for the previously recognized consensus amino acid sequence for CRM1 dependent NESs but also was the basis for a thorough mutational analysis that more clearly defined the amino acid requirements at five $\Phi$ positions. These experiment in combination with the available structures provided a clearer picture of the properties that render a linear amino acid sequence into a faithful CRM1 binder. We wanted to make use of the gained information to develop a prediction tool that would identify and score potential NESs within a give sequence.

\subsection{A NEW NES PREDICTION ALGORITHM}

The widely accepted consensus amino acid sequence for CRM1-dependent nuclear export signals (NESs) $\Phi-x_{(2-3)}-\Phi-x_{(2-3)}-\Phi-x-\Phi$ ( $\Phi$ for hydrophobic residues, $x$ for any amino acid) is better understood in the context of the later solved CRM1-RanGTP-Cargo crystal structures (Dong et al., 2009; Monecke et al., 2009; Güttler et al., 2010).

We wanted to apply the new experimental findings to generate an improved NES prediction tool. To achieve this goal, we focused on the NES prototypes with the same $\Phi$ residue spacing as in these crystal structures and considered a previously published systematic mutational analysis for each of these $\Phi$ positions (Güttler et al., 2010). The outcome of the latter study resulted in a scoring matrix to estimate CRM1 binding strength of a given sequence.

The consensus NES definition contains several critical hydrophobic residues. Since hydrophobic residues are often buried in the folded core of the protein structure, there is a high probability of finding NES hits that are not accessible for CRM1 interaction. To sort out such potential false positives, we applied two types of filtering. First we predicted the disorder propensity for the found hit, and the 6 amino acids before and after that region. This prediction is used for assessing the possibility of the hit being exposed for an interaction. Second, protein sequence is searched for domain homology since folded domains are less likely to contain a disordered stretch of amino acids that can act as an NES. At the end, high scoring NESs that are not in a folded domain and that have high disorder propensity were considered as good hits. 


\subsubsection{NES Consensus}

Crystal structures of CRM1 with NESs show that there are at least two different arrangements of 5

Ф residues that can fit into the hydrophobic cleft (Güttler et al., 2010). The first one is the more common NES pattern that is in agreement with the PKI NES $\Phi$ residue arrangement. The second one follows the REV NES $\Phi$ residue arrangement, and described as a new class of NES consensus for the first time. I will refer to these two types as PKI-type and REV-type NES.

\subsubsection{PKI-type NES consensus}

To scan the given protein sequences for NES hits, a pattern-matching algorithm called regular expression is used. To construct the PKI-type regular expression, following statements are used.

- PKI type $\Phi$ residues follow a $\Phi_{1}-\mathrm{x}_{(3)}-\Phi_{2}-\mathrm{x}_{(2-3)}-\Phi_{3}-\mathrm{x}-\Phi_{4}$ spacing (la Cour et al., 2004) (Güttler et al., 2010)

- Proline residues are not allowed in the spacer regions between $\Phi_{1}$ and $\Phi_{4}$ (Kosugi et al., 2008).

- $\Phi_{0}$ and neighboring negatively charged amino acids contribute positively to the binding (Güttler et al., 2010).

These statements were combined into the PKI-type NES regular expression (Figure 7-1).

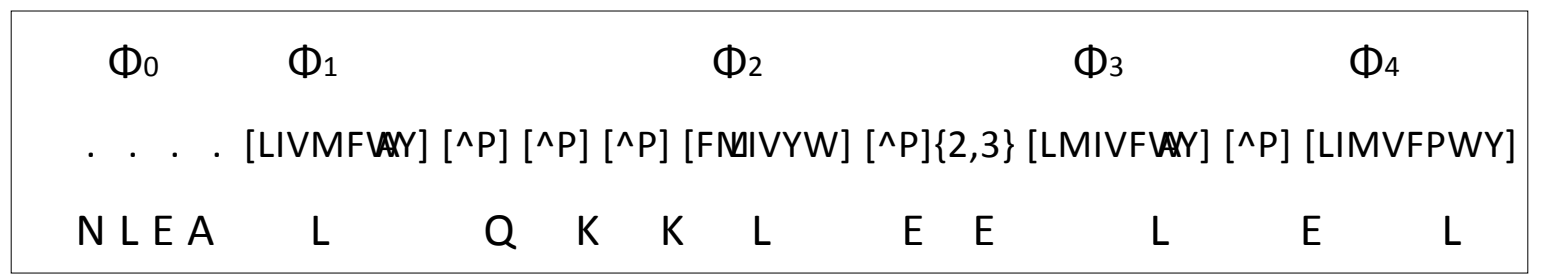

Figure 7-1 Regular expression for PKI-type NES pattern and an example NES

Each bracket-enclosed expression represents a position with allowed amino acids. Dot (.) represents any residue. Residue after ' $\wedge$ ' sign is not allowed at that position. Curly brackets indicate the allowed repeat numbers for the previous pattern (e.g. $[\wedge P](2,3)$ means 2 to 3 amino acids stretch without any proline). Underneath the regular expression the NES from Map kinase kinase 1 is placed with matching positions.

The first 4 amino acids including the $\Phi_{0}$ position did not have any prerequisites during the pattern search, since any amino acid (represented by ' ' in a regular expression) can be matched. Contribution of these residues was graded later in the NES Score. Allowed amino acids in the $\Phi$ positions are explained in the NES Score section.

\subsubsection{REV-type NES consensus}

Early studies tried to "squeeze" the REV NES into a PKI-type consensus. The actual CRM1-Rev-NES structure revealed however a different binding conformation between $\Phi 0$ and $\Phi 2$ and $\Phi_{1}$ pocket was occupied by a proline and not by a more typical hydrophobic amino acid (Güttler et al., 2010). 
A regular expression by this new structural definition was constructed for REV-type NES consensus (Figure 7-2).

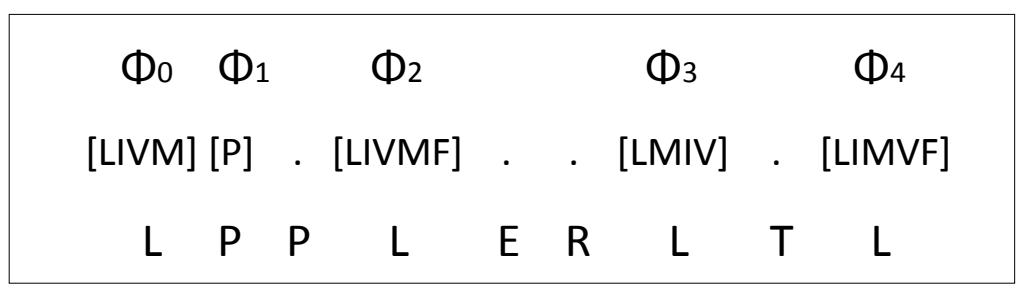

Figure 7-2 Regular expression for REV-type NES pattern and an example NES

Each bracket-enclosed expression represents a position with allowed amino acids. Dot (.) represents any residue. Underneath the regular expression the NES from Rev protein is placed with matching positions.

NES score was calculated only for PKI-type NES hits, and for REV-type NES $\Phi$ positions, only a limited set of favored hydrophobic amino acids were allowed.

\subsubsection{NES Score}

An NES scoring scheme for PKI-type NES hits was designed based on previously published CRM1 binding assay with point mutants of PKI NES (Güttler et al., 2010). For $\Phi$ residues 1 to 4, each position was given an incremental score (S1 to S4) of 1 to 10 , based on the ranking of amino acid preference. These amino acids include tyrosine, tryptophan, phenylalanine, and alanine in addition to the previously recognized hydrophobic residues leucine, valine, methionine, and isoleucine. Alanine is only accepted as $\Phi_{1}$ or $\Phi_{3}$ residue (Figure 7-3).

Previous NES consensus definitions used $4 \Phi$ positions $\left(\Phi_{1}\right.$ to $\left.\Phi_{4}\right)$, which were important for CRM1 interaction. With the previously published crystal structures of NES bound CRM1, a previously unrecognized NES position was defined and named $\Phi_{0}$ (Güttler et al., 2010). Although it was defined recently, an analysis showed that $76 \%$ of validated NES, which fit into previous NES consensus, has a hydrophobic residue in $\Phi_{0}$ position (Xu et al., 2012b). This position clearly contributes to binding, and allows the construction of high affinity CRM1 binders. 
A) Scoring $\Phi$ residues 1 - 4

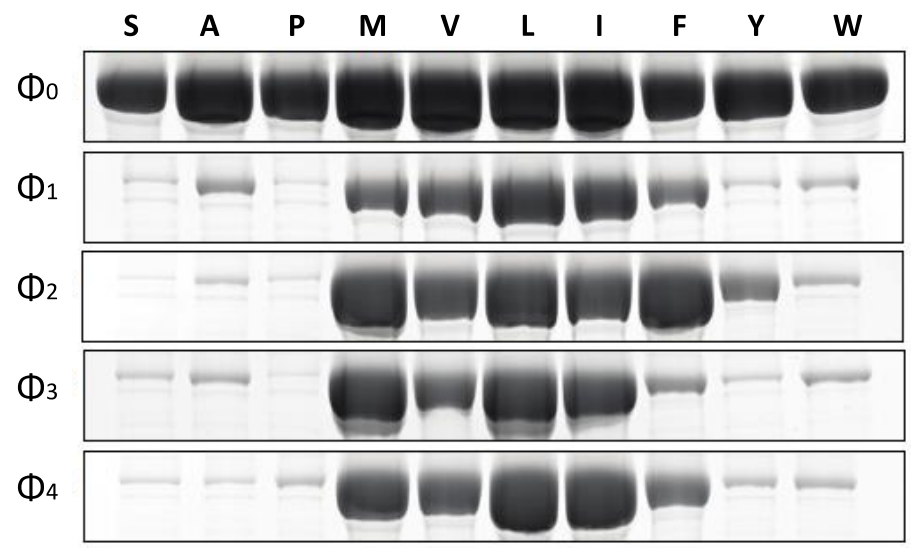

$L>I>V M>F>A>W Y$

$\begin{array}{llllll}10 & 8 & 6 & 4 & 2 & 1\end{array}$

$\mathrm{S}_{1}$

$\mathrm{FML}>\mathrm{IV}>\mathrm{Y}>\mathrm{W}$

$\begin{array}{llll}10 & 8 & 3 & 1\end{array}$

$\mathrm{S}_{2}$

$\mathrm{L} M>\mathrm{I}>\mathrm{V}>\mathrm{F}>\mathrm{W}$ A $>$ Y

$\begin{array}{llllll}10 & 9 & 7 & 3 & 2 & 1\end{array}$

$\mathrm{S}_{3}$

$\mathrm{L}>\mathrm{I}>\mathrm{M}>\mathrm{V}>\mathrm{F}>\mathrm{P}$ Y W

$\begin{array}{llllll}10 & 9 & 8 & 7 & 5 & 1\end{array}$

$\mathrm{S}_{4}$

B) Scoring $\Phi_{0}$ and neighbouring aa

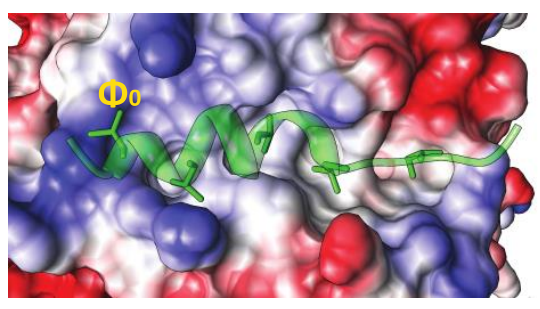

\begin{tabular}{|lll|}
\hline & $x_{1}$ & $x_{2} x_{3}$ \\
PKI NES & N S NE L A LKLA G LDI \\
\hline
\end{tabular}

$\begin{array}{cccccc}X_{1} & \Phi_{0} & X_{2} & X_{3} & \text { Min Score } 1 & \text { So } \\ \text { ED } & \text { IVML }>\text { FAYW } & \text { ED } & \text { ED } & & \end{array}$

$0.5+0.5 \quad 0.25+0.5+0.5 \quad$ Max Score 2

C) PKI-type NES score

NES Score $=S_{0} \times S_{1} \times S_{2} \times S_{3} \times S_{4} \quad$ Min Score 1 Max Score 20000

Figure 7-3 Scoring Scheme for PKI-type NESs

A) Correlation between $\Phi$ position occupation and CRM1-binding strength and conversion of this correlation into scores (Güttler et al., 2010). B) PDB structure entry 3NBY. CRM1 surface colored according to coulomb potential (red is negative and blue is positive) and $\Phi_{0} \mathrm{~L}$ PKI sequence backbone is colored transparent green, and pocket fitting $\Phi$ residue side chains are colored solid green. Scoring scheme for $\Phi_{0}$ and negative residues around it. C) Calculation of NES score based on $\Phi$ position specific scores.

Negatively charged residues around $\Phi_{0}$ also contribute to this binding with electrostatic interactions, evident from the positive charges around $\Phi_{0}$ binding pocket, and also from previous studies (Figure 7-3B) (Güttler et al., 2010). Since contribution of this part was not as crucial as the other $\Phi$ residues, its effect to the score was limited with a coefficient of 2 . If the $S_{0}$ score was less that 1 , it was overridden by 1 to not to affect the final score negatively.

The final PKI-type NES score was calculated by multiplying all 5 sub-scores to represent the cooperative binding of $\Phi$ pockets. This can yield score of 1 as minimum and score of 20000 as maximum.

\subsubsection{Disorder Propensities}

For an NES to bind CRM1, we reasoned that not only the NES itself but also a small region following and preceding the actual NES should have disorder tendencies. Following this reasoning 
we analyzed three regions for disorder propensity by IUPred; the six amino acids before the hit, the candidate NES, and the six amino acids after the hit. IUPred gives a disorder propensity value for each amino acid ranging from 0 (complete order) to 1 (complete disorder), and these three regions got one value each by averaging the disorder propensity over the analyzed region. If the NES hit is at the extreme $\mathrm{N}$ or $\mathrm{C}$-terminus, the disorder propensity is set to 1 for the preceding or proceeding part.

For simplicity, disorder values were categorized into three sections. The first category was indicated by " 1 " and covered averaged disordered propensities of 0 to 0.25 . Second category was indicated by "2" and covered averaged disordered propensities of 0.25 to 0.5 . Third category was indicated by " 3 " and covered averaged disordered propensities of 0.5 to 1.0. IUPred regards values lower than 0.5 as order and values higher than 0.5 as disorder. The lower half was separated into two categories because previous studies showed that a large portion of linear motifs also resided in the second range (Fuxreiter et al., 2007).

Additional information was fetched from SMART domain database (Schultz et al., 1998). Since not all annotated domains are folded domains and prediction of exact domain borders are not accurate, such domain prediction was used with caution. When multiple sequences were analyzed, domain prediction was exempted from constraints. It was used as a visual inspection tool of individual hits, since a final reasoning requires analysis of the predicted domains.

\subsubsection{Evaluation of PKI-type NES prediction}

We wanted to know if the algorithm would allow prediction of functional NESs within a given amino acid sequence. To this end we selected 11 proteins the NES of which have been experimentally characterized before by others. Their primary sequences were retrieved from databases and fed into the NES prediction algorithm. Sequence analysis revealed putative PKItype NESs, which were subsequently ranked according to our scoring criteria. Interestingly, highest scoring NES hits largely matched the sequences that have been validated experimentally. This indicated that the algorithm was indeed capable of predicting functionally relevant NES.

The PKI-type NES prediction algorithm was written in Python (12.1.1), which is a programming language that is widely used by the bioinformatics community and for which many code libraries are already available. The Input file is a fasta formatted protein sequence or several sequences. The algorithm iterates over the given sequences and outputs the predicted NES borders and sequences with disorder propensity, domain prediction, an NES score for each hit sequence. An example output is shown in Figure 7-4 for human MAP kinase kinase 1 (MP2K1_Human). 


\begin{tabular}{|c|c|c|c|c|c|c|c|}
\hline \multicolumn{8}{|c|}{ MP2K1_HUMAN } \\
\hline \multicolumn{3}{|c|}{ Disorder } & Sequence & Start & End & Domain & NES Score \\
\hline B & NES & A & & & & & \\
\hline 3 & 2 & 2 & NLEALQKKLEELEL & 29 & 42 & n.i.d. & 10000 \\
\hline 1 & 1 & 1 & SGLVMARKLIHLEI & 90 & 103 & in S_TKc & 5400 \\
\hline 1 & 1 & 1 & GLVMARKLI HLEIKP & 91 & 105 & in S_TKc & 144 \\
\hline 1 & 1 & 1 & IKPAIRNQI IRELQV & 103 & 117 & in S_TKc & 4480 \\
\hline 1 & 1 & 1 & CNSPYIVGFYGAFY & 121 & 134 & in S_TKc & 20 \\
\hline 1 & 1 & 1 & I PEQILGKVSIAVI & 161 & 174 & in S_TKc & 1152 \\
\hline 1 & 1 & 1 & ILGKVSIAVIKGLTY & 165 & 179 & in S_TKc & 480 \\
\hline 2 & 1 & 1 & THYSVQSDIWSMGL & 238 & 251 & in S_TKc & 4800 \\
\hline 2 & 1 & 2 & QSDIWSMGLSLVEM & 243 & 256 & in S_TKc & 560 \\
\hline 2 & 1 & 2 & DIWSMGLSLVEMAV & 245 & 258 & in S_TKc & 4200 \\
\hline 3 & 2 & 1 & RPPMAIFEILDYIV & 305 & 318 & in S_TKc & 140 \\
\hline 1 & 1 & 1 & ERADLKQLMVHAF I & 348 & 361 & in S_TKc & 1800 \\
\hline 1 & 1 & 2 & EEVDFAGWLCSTIGL & 367 & 381 & n.i.d. & 3600 \\
\hline
\end{tabular}

Figure 7-4 An output example from PKI- type NES prediction

MP2K1 is the abbreviation for MAP kinase kinase 1 and was shown to have an N-Terminal NES (Fukuda et al., 1996). From the three disorder values (B) represents the six amino acids before the NES, (NES) represents the predicted hit, and (A) represents the six amino acids after NES. S_TKc is the abbreviation for SMART domain Serine/Threonine protein kinases, catalytic domain. 'n.i.d' stands for 'not in any domain'. Hits with a NES disorder prediction of 1 are shaded gray. For other hits, putative $\Phi$ positions are marked bold.

Performance of the NES score and disorder filtering for PKI-type NES prediction was evaluated on a set of previously defined NES dependent CRM1 cargos. 11 proteins of NES instance examples from ELM database were used for evaluation (Table 7-1).

\begin{tabular}{|c|c|c|}
\hline Protein & NES Sequence & Reference \\
\hline $\begin{array}{l}\text { Spn1_Human } \\
\text { Snurportin } 1\end{array}$ & ${ }^{1}$ MEELSQALASSFSV $^{14}$ & (Monecke et al., 2009) \\
\hline $\begin{array}{l}\text { Apc_Human } \\
\text { Adenomatous polyposis coli protein }\end{array}$ & ${ }^{64}$ GQIDLLERLKELNL ${ }^{77}$ & (Henderson, 2000) \\
\hline $\begin{array}{l}\text { Ccnb1_Xenla } \\
\text { G2/mitotic-specific cyclin-B1 }\end{array}$ & ${ }^{104}{ }_{\text {LPDELCQAFSDVLI }}^{117}$ & (Yang et al., 1998) \\
\hline $\begin{array}{l}\text { Ctnd1_Human } \\
\text { Catenin delta-1 }\end{array}$ & ${ }^{940}$ GQESLEEELDVLVL ${ }^{953}$ & (van Hengel et al., 1999) \\
\hline $\begin{array}{l}\text { Ipka_Human (PKI) } \\
\text { cAMP-dependent protein kinase inhibitor } \alpha\end{array}$ & ${ }^{34}$ NSNELALKLAGLDI $^{47}$ & (Johnson et al., 1999) \\
\hline $\begin{array}{l}\text { Rang_Human } \\
\text { Ran-binding protein } 1\end{array}$ & ${ }^{176}$ HAEKVAEKLEALSV ${ }^{189}$ & (Richards et al., 1996) \\
\hline
\end{tabular}




\begin{tabular}{|c|c|c|}
\hline $\begin{array}{l}\text { Mp2k1_Xenla } \\
\text { MAP kinase kinase } 1\end{array}$ & ${ }^{29}$ NLEALQKKLEELEL $^{42}$ & (Fukuda et al., 1996) \\
\hline $\begin{array}{l}\text { Nep_I34a1 } \\
\text { Nuclear export protein }\end{array}$ & ${ }^{8}$ SFQDILLRMSKMQL ${ }^{21}$ & (O'Neill et al., 1998) \\
\hline $\begin{array}{l}\text { P53_Human } \\
\text { Cellular tumor antigen p53 }\end{array}$ & ${ }^{336}$ ERFEMFRELNEALEL ${ }^{350}$ & (Stommel et al., 1999b) \\
\hline $\begin{array}{l}\text { Per2_Mouse } \\
\text { Period circadian protein homolog } 2\end{array}$ & ${ }^{456}$ SVQELTEQIHRLLM ${ }^{469}$ & (Vielhaber et al., 2001) \\
\hline $\begin{array}{l}\text { Rex_Htl1a } \\
\text { Protein Rex }\end{array}$ & ${ }^{78}$ SMDALSAQLYSSLSL $^{92}$ & (Bogerd et al., 1996) \\
\hline
\end{tabular}

Table 7-1 Validated NES containing proteins

11 proteins were previously shown to have a CRM1 dependent NES. Regions of NESs are indicated with the starting and ending amino acid numbers, and the NES sequence. NES sequences were validated by the indicated reference. Putative $\Phi$ residues are marked bold.

These hits were evaluated for PKI-type NES content with the prediction algorithm. When NES scores from each protein were analyzed, the score from the true hit was the highest score or was one of the highest (Figure 7-5). For proteins RanBP1, cAMP-dependent protein kinase inhibitor $\alpha$, and Rex there was only one hit identified. For all three cases this was the previously validated NES.

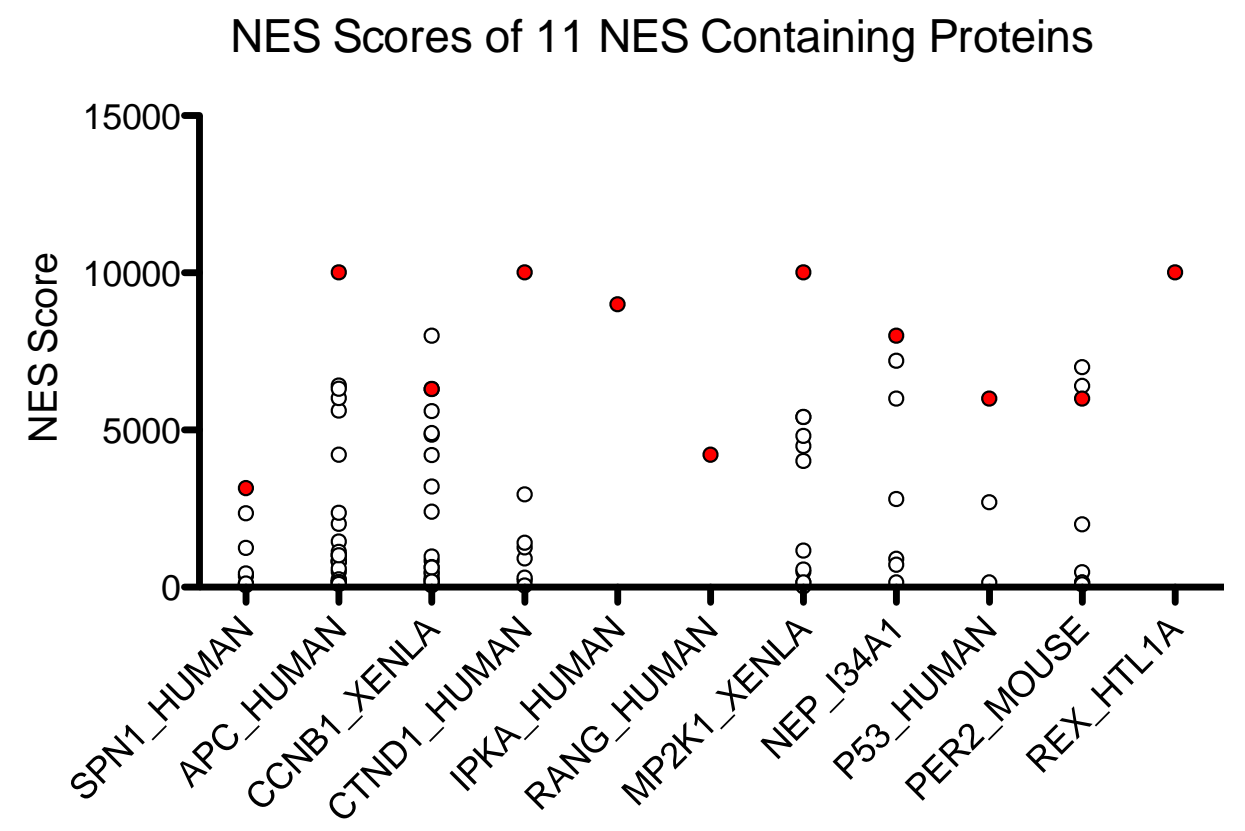

Figure 7-5 NES Scores of the PKI-type hits of the 11 selected proteins

NES scores were calculated for predicted PKI-type NES hits. Each hit from each protein is represented with a circle. The previously validated NESs are indicated with red color. 


\section{Disorder Propensities of NES Hits}

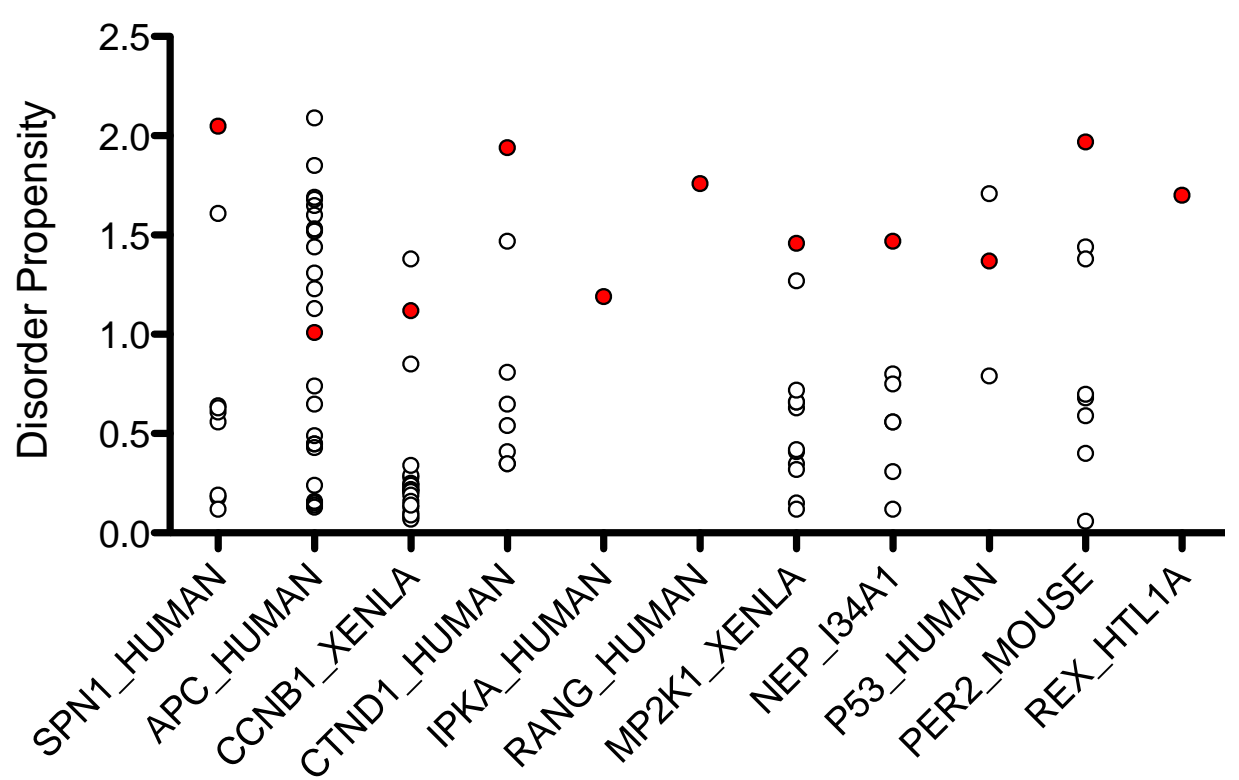

Figure 7-6 Aggregated disorder propensities of the PKI-type hits of the $\mathbf{1 1}$ selected proteins

The averaged disorder propensities of 6 amino acids before the hit, the NES hit, and 6 amino acids after the hit were summed (minimum of 0 and maximum of 3). NES scores were calculated for predicted PKI-type NES hits. Each hit from each protein is represented with a circle. The previously validated NESs are indicated with red color.

To analyze the disorder propensities of the hits, not only the hit itself but also the 6 amino acids before and after were considered. This led to an aggregated disorder score that had a value between 0 (order) and 3 (disorder). Disorder values for the previously confirmed hits were among the top ones except protein APC (Figure 7-6). APC is a very large protein with 2843 amino acids. Cterminal half has a high disorder propensity, and it is predicted to have many NES hits with weak NES score. If all hits from 11 proteins are plotted both with NES scores and disorder propensities, one can see the clear distinction between true and false hits (Figure 7-7).

NES Score vs Disorder Propensity

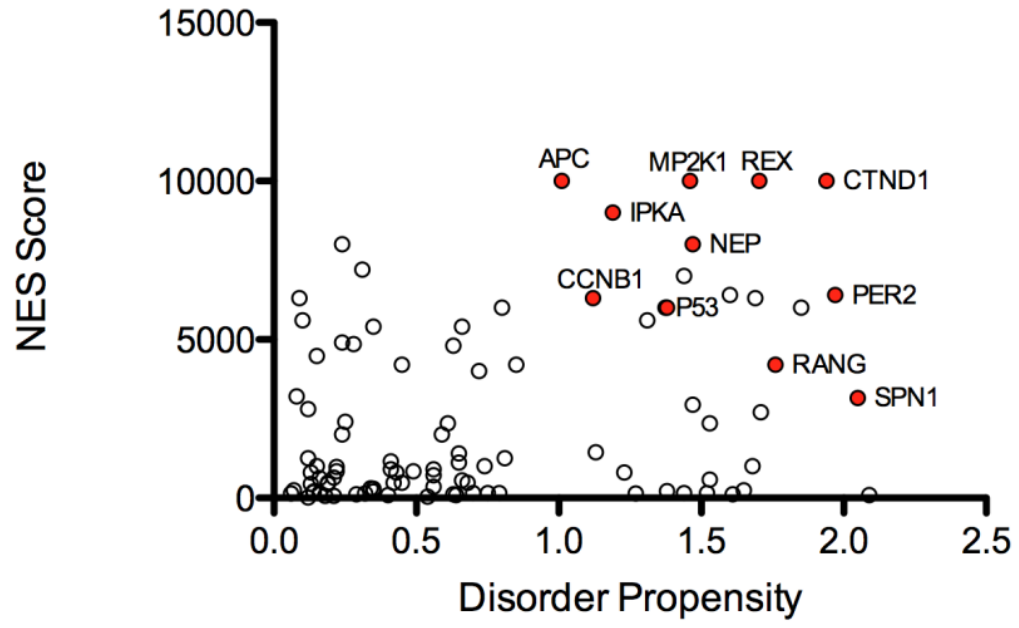

Figure 7-7 NES scores and disorder propensities of each PKI-type NES hit 


\subsubsection{Evaluation of REV-type NES prediction}

The CRM1 crystal structure with the bound Rev NES revealed an unusual placement of $\Phi$ residues in CRM1 hydrophobic cleft. This was a new NES definition, so we wanted to see if there were previously annotated NES, which would fit into REV-type NES.

A second algorithm was written with Python (12.1.2). The algorithm iterates over the given sequences and outputs the predicted NES borders and sequences with disorder propensity, domain prediction for each hit sequence. Since there was no $\Phi$ position mutation study for this type of NES, thus we did not calculate an NES score for this type. Instead only a limited selection of hydrophobic residues was allowed at each $\Phi$ position (Figure 7-2).

To extend the number of examples and show that this pattern also exists in other proteins, 236 NES containing proteins from the curated database NESdb (Xu et al., 2012a) were analyzed for their REV-type NES match. The hits matching with the annotated NESs are listed.

\begin{tabular}{|c|c|c|}
\hline Protein & NES Sequence & Reference \\
\hline $\begin{array}{l}\text { Rev_Hv1h3 } \\
\text { Protein Rev }\end{array}$ & ${ }^{75}$ LPPLERLTL $^{83}$ & $\begin{array}{l}\text { (Meyer and Malim, 1994) } \\
\text { (Güttler et al., 2010) }\end{array}$ \\
\hline $\begin{array}{l}\text { Tf3a_Anaae } \\
\text { Transcription factor IIIA }\end{array}$ & ${ }^{330}$ LPVLENLTL $^{338}$ & (Fridell et al., 1996) \\
\hline $\begin{array}{l}\text { Ddx6_Xenla } \\
\text { ATP-dependent RNA helicase ddx6 }\end{array}$ & ${ }^{151}$ IPLLERLDL $^{159}$ & $\begin{array}{l}\text { (Smillie and Sommerville, } \\
\text { 2002) }\end{array}$ \\
\hline $\begin{array}{l}\text { gi } 159024820 \\
\text { Nonstructural protein NS5 }\end{array}$ & ${ }^{335}$ VPMVTQMAM $^{343}$ & (Rawlinson et al., 2009) \\
\hline $\begin{array}{l}\text { Nf212_Human } \\
\text { NF-E2-related factor } 2\end{array}$ & ${ }^{194}$ IPELQCLNII $^{202}$ & (Li et al., 2006) \\
\hline $\begin{array}{l}\text { Q99AM3_HHV8 } \\
\text { B-cell specific latent nuclear protein }\end{array}$ & ${ }^{552}$ VPLVIKLRL $^{560}$ & (Munoz-Fontela et al., 2005) \\
\hline $\begin{array}{l}\text { Fbx7_Human } \\
\text { F-box only protein } 7\end{array}$ & ${ }^{326}$ LPDVFGLVV $^{334}$ & (Nelson and Laman, 2011) \\
\hline
\end{tabular}

Table 7-2 REV-type NESs from NESdb

Interestingly there were 6 proteins with REV-type NESs other than Rev protein. This suggests that REV-type NESs might indeed represent a more general binding mode that is used by several proteins. 


\subsection{IDENTIFICATION OF NES ON EIF2 $\beta$}

For certain known CRM1 cargoes, the hitherto available prediction tools failed to identify a bona fide NES. We reasoned that our new algorithm might be more powerful than previous tools and used it to predict putative NESs on two proteins that are of general interest for our lab, human eIF2 $\beta$ and Schizosaccharomyces pombe Rna1p.

The first protein that was analyzed with the prediction algorithm was human eukaryotic translation initiation factor $\underline{2}$ subunit $\underline{\beta}$ (elF2 $\beta$ ). elF $2 \beta$ is part of the trimeric elF2 complex that is responsible for bringing the initiator methionine-tRNA to $40 S$ ribosomal subunit. eIF $2 \beta$ was shown to accumulate in the nucleus upon Leptomycin B treatment, indicating CRM1 dependent nuclear exclusion (Bohnsack et al., 2002). Since existing bioinformatics tools failed to predict testable NESs on elF2 $\beta$, Chandini Kadian from our lab was trying to experimentally narrow down the CRM1 interaction site on this protein.

\subsubsection{Prediction of elF2 $\beta$ NES hits}

The primary sequence of the protein was analyzed with the PKI and REV-type prediction algorithms. There was no REV-type hit, but 3 PKI-type hits were predicted (Figure 7-8). Out these 3 hits, the second one was considered as a significant hit since it had the highest NES score (2880) and a high disorder propensity. This NES was not noticed before, because it had an alanine residue in its $\Phi_{1}$ position, and alanine was not considered as a suitable amino acid for $\Phi$ positions by previous prediction tools.

\begin{tabular}{|c|c|c|c|c|c|c|c|}
\hline \multicolumn{8}{|c|}{ IF2B_HUMAN } \\
\hline & sord & & Sequence & Start & End & Domain & NES Score \\
\hline B & NES & $A$ & & & & & \\
\hline 3 & 2 & 2 & RKKDASDDLDDLNF & 62 & 75 & n.i.d. & 1000 \\
\hline 2 & 2 & 3 & DIDEAEEGVKDLKI & 90 & 103 & n.i.d. & 2880 \\
\hline 2 & 2 & 2 & RDYTYEELLNRVFNI & 172 & 186 & n.i.d. & 270 \\
\hline
\end{tabular}

Figure 7-8 Prediction of NES hits of human elF2 $\beta$

IF2B is the Uniprot ID for eukaryotic translation initiation factor $\underline{2}$ subunit $\underline{\beta}$ (elF2 $\beta$ ). From the 3 disorder values (B) represents the 6aa before NES, (NES) represents the predicted hit, and (A) represents the 6aa after NES. ' $n$.i.d' stands for 'not in any domain'. Putative $\Phi$ residues are marked bold.

\subsubsection{Validation of elF2 $\beta$ NES hit}

We also analyzed the candidate NES for conservation among close species. To see the variance of NES hit sequence among vertebrate homologs, human eIF2 $\beta$ sequence was blasted against vertebrates, and aligned with the top hits (Figure 7-9). All hits followed the allowed sequences for Ф positions except one. Xenopus laevis had 2 homologs, and one of them (Q619h4_xenla) had a threonine residue instead of an alanine in the $\Phi_{1}$ position. 


\begin{tabular}{|c|c|c|}
\hline & & 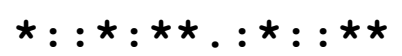 \\
\hline & UMAN & LKI \\
\hline$\overline{\mathrm{P}} 6$ & JAB & LDEAEEGVKDLKI \\
\hline A6I 4 & PANTR & DIDEAEEGVK \\
\hline QK76 & PANTR & DIDEAEEGVKDI \\
\hline G3RHP2 & GORGO & DIDEAEEGVKDLI \\
\hline FW4 8 & MACMU & DIDEAEEGVKDLI \\
\hline G7PGK 4 & MACFA & DIDEAEEGVKDLF \\
\hline L9L7A8 & TUPCH & DIDEAEEGVKDLKI \\
\hline L5K0T8 & PTEAL & DIDEAEEGVKDMF \\
\hline I3MCD2 & SPETR & DIDEAEEGVKDLKI \\
\hline$I F 2 B$ & ŌVIN & DIDEAEEGIKDLKI \\
\hline L8I $4 \overline{\mathrm{H}} 9$ & BOSMU & DIDEAEEGIKDLKI \\
\hline 47 & CANFA & DIDEAEEGVKDLKI \\
\hline G1P4 I 4 & MYOLU & DIDEAEEGVKDLKI \\
\hline G3THD5 & LOXAF & DIDEAEEGIKDLKI \\
\hline HOWIJ7 & ОTOGA & DIDEAEEGVKDLF \\
\hline G1LR6 6 & AILME & DIDEAEEGVKDLKI \\
\hline G5BPT 9 & HETGA & DIDEAEEGIKDLKI \\
\hline F1S4Y8 & PIG & DIDEAEEGVKDLKI \\
\hline L5LUF5 & MYODS & DIDEAEEGVKDLKI \\
\hline IF2B M & ŌUSE & DIDEAEEAIKDVKI \\
\hline Q6P6 $8 \overline{8} 5$ & RAT & DIDEAEEAIKDVKI \\
\hline G3HAN5 & CRIGR & DIDEAEEGVKDLKI \\
\hline M3Z296 & MUSPF & DIDEAEEGVKDLKI \\
\hline K9IZY5 & DESRO & DIDEAEEGVKDLKI \\
\hline F7DE 88 & HORSE & DIDEAEEGVKDLKI \\
\hline IF2B $R$ & $\overline{\mathrm{A}} \mathrm{BIT}$ & DIDEAEEGVKDLKI \\
\hline F6SF $\bar{K} 4$ & CALJA & DIDEAEEGVKDLK \\
\hline Q6P7N2 & XENTR & DLDEAEEGVKNLK] \\
\hline Q6Q4H9 & XENLA & DLEETEEGVKNLK: \\
\hline & $\mathbf{F}$ & DEAEEGVKN \\
\hline
\end{tabular}

Figure 7-9 Alignment of hs elF $2 \beta$ protein sequence with vertebrate orthologs

eIF $2 \beta$ orthologs were retrieved from Uniprot database and alignment was done with ClustalX 2.0 default settings. $\Phi$ residues are indicated with orange background. Proteins are named with Uniprot IDs.

To validate this putative NES, it was expressed in E.coli as His10-ZZ-Tev fusion to use in the RanGTP dependent CRM1 binding assays. To test the effect of the alanine in the $\Phi_{1}$ position, a $\Phi_{1} A \Rightarrow L$ mutant version of the putative NES was designed.

To tests its CRM1 binding, residues corresponding to Xenopus laevis elF2 $\beta$ NES hit were also expresses as His10-ZZ-Tev fusion. Human eIF2 $\beta$ NES hit, the $\Phi_{1} A \Rightarrow L$ mutant, the $\Phi_{1} A \Rightarrow T$ Xenopus laevis ortholog, and the PKI NES were incubated with mmCRM1 either in the presence or absence of $\operatorname{Ran}_{5-180}$ Q69L GTP. 
A)

hs PKI

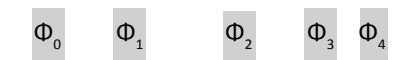

hs elf2 $\beta$

${ }^{34} \mathrm{NSNELALKLAGL} \mathrm{L} \mathrm{I}{ }^{47}$

hs elF2 $\beta$ A94L

${ }^{90}$ D I DEAEEGVKDLK I ${ }^{103}$

$x \mid$ elf $2 \beta$

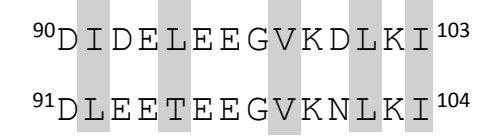

B)

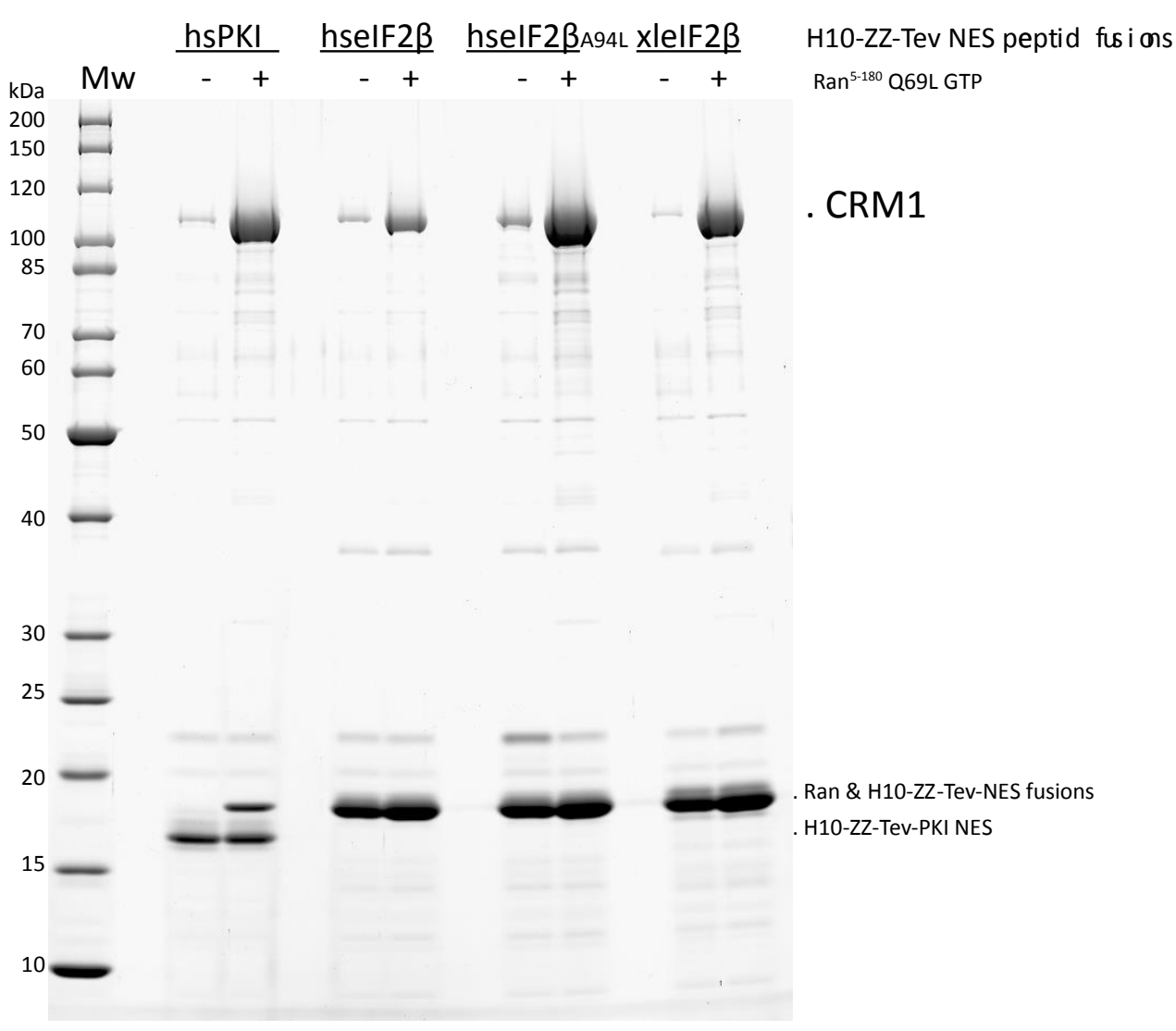

Figure 7-10 RanGTP dependent mmCRM1 binding of elF2 $\beta$ NES hits

The binding reactions were performed in $500 \mu$ volume with $2 \mu \mathrm{M}$ mmCRM1 and $2 \mu \mathrm{M}$ His10-ZZ-Tev-NES fusion. For the reactions with Ran, $3 \mu \mathrm{M} \operatorname{Ran}_{5-180}$ Q69L GTP was added. Final buffer concentration was adjusted to $50 \mathrm{mM}$ Tris/ $\mathrm{HCl}$ $7.5,120 \mathrm{mM} \mathrm{NaCl}, 2 \mathrm{mM} \mathrm{Mg}(\mathrm{OAC})_{2}, 5 \mathrm{mM} \mathrm{DTT}$. After 2 hours at $4^{\circ} \mathrm{C}$, ZZ-affibody beads were added to pull down the NES peptides and the bound proteins.

A) Alignment of PKI NES to human and Xenopus laevis elF2 $\beta$. Ф residues are indicated above the PKI NES and their alignments with NES hits are shaded gray. Start and end residue numbers of NESs are indicated in the full-length protein context. B) SDS-PAGE analysis of RanGTP dependent mmCRM1 binding of NES hits. H10 stands for N-terminal 10 histidine residues, Z (in ZZ) stands for IgG-binding domain of the Staphylococcal protein A, and Tev stands for Tobacco Etch Virus protease recognition sequence. Protein ladder is abbreviated with ' $\mathrm{Mw}$ ' for molecular weight, and protein sizes are indicated on the left side of the corresponding bands. Samples without RanGTP are indicated with a '-' sign, and samples with RanGTP are indicated with ' + ' sign.

Beads were eluted with $100 \mu \mathrm{l}$ SDS sample buffer, and $10 \mu \mathrm{l}$ of elution was analyzed with SDS-

PAGE. PKI NES served as the positive control and showed RanGTP dependent CRM1 interaction.

The candidate NES of eIF2 $\beta$ was weaker than PKI in the CRM1 interaction, but still showed a significant binding. This binding was enhanced when the $\Phi_{1}$ residue was mutated from alanine to 
leucine, indicating that this was truly a $\Phi$ pocket binding position. Interestingly the Xenopus laevis homolog of the NES was also functional with a threonine in $\Phi_{1}$ position (Figure 7-10).

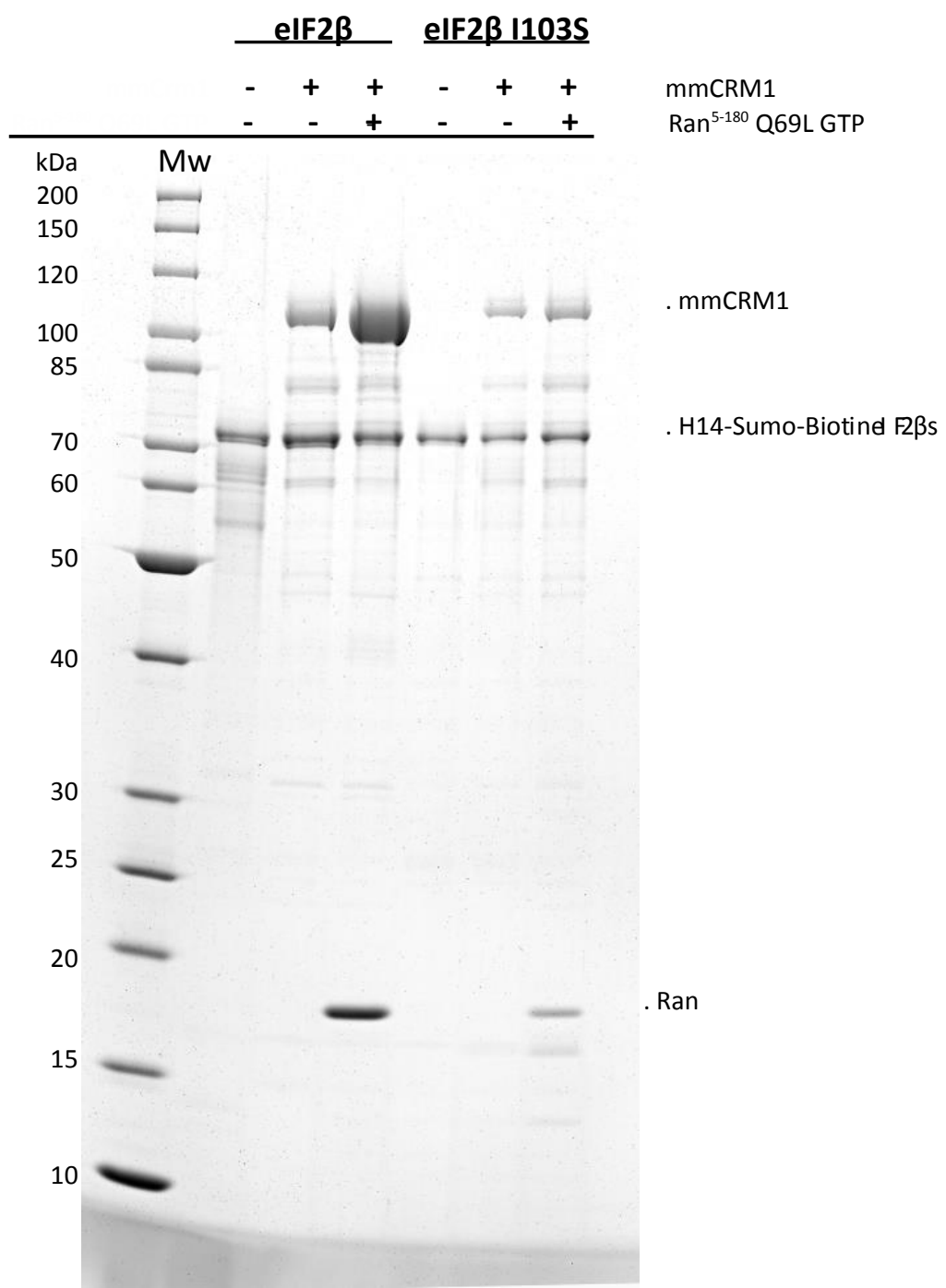

Figure 7-11 RanGTP dependent mmCRM1 binding of wild type and NES mutant of human elF2 $\beta$

$\mathrm{H} 10$ stands for N-terminal 10 histidine residues, Z (in ZZ) stands for IgG-binding domain of the Staphylococcal protein A. Protein ladder is abbreviated with ' $M w^{\prime}$ ' for molecular weight, and protein sizes are indicated on the left side of the corresponding bands. Samples without RanGTP are indicated with a '-' sign, and samples with RanGTP are indicated with ' + ' sign.

It was also important to test the validity of the NES in the context of full-length protein. To test this, the $\Phi_{4}$ position was mutated from isoleucine to serine, and both the wild type human elF2 $\beta$ and the I103S mutant were expressed as biotinylated full-length proteins. Proteins were incubated with mmCRM1 either in the presence or absence of RanGTP, and the final salt concentration was adjusted to $100 \mathrm{mM} \mathrm{NaCl}$. Reaction was incubated with Streptavidin-Agarose beads. Elution was done with $37^{\circ} \mathrm{C}$ SDS sample buffer which is enough for elution of mmCRM1 and Ran but cannot fully disrupt streptavidin-biotin interaction. Elutions were analyzed with SDSPAGE (Figure 7-11). 
Taken together, with the prediction algorithm we identified 3 NES hits for human elF2 $\beta$ and analyzed the most prominent hit in terms of NES score. This NES hit was indeed able to bind CRM1. By mutation of the $\Phi 4$ position in the full-length protein, we were able to abolish the interaction of full-length elF2 $\beta$ with CRM1. The predicted hit had and unusual alanine residue in $\Phi_{1}$, confirmed by the leucine mutant that had a stronger affinity for CRM1. Thus, our algorithm proved useful to predict a functional NESs that has escaped previous attempts of bioinformatics analysis. 


\subsection{IDENTIFICATION OF NES ON spRna1p}

Second protein of interest was spRna1p, which is the RanGAP ortholog in Schizosaccharomyces pombe. spRna1p has an $\mathrm{N}$-terminal leucine rich repeat (LRR) domain followed by a poly glutamic acid region (Figure 6-5). It is kept cytoplasmic in S.pombe (Melchior et al., 1993b). Previous studies suggest Crm1 mediated export but the NES region was not experimentally identified for spRna1p.

\subsubsection{Previously suggested NESs are buried in the structure}

The Saccharomyces cerevisiae homolog of Rna1p (scRna1p) was previously shown to interact with Crm1 and also two NESs were identified. By homology, 2 corresponding NESs were also suggested for spRna1p (Feng et al., 1999). In the same year, the N-terminal LRR region of S.pombe Rna1p protein was crystallized (Hillig et al., 1999). In order to see if the suggested regions would serve as an NES also in spRna1p, we analyzed the crystal structure for the accessibility of the previously suggested NES regions.

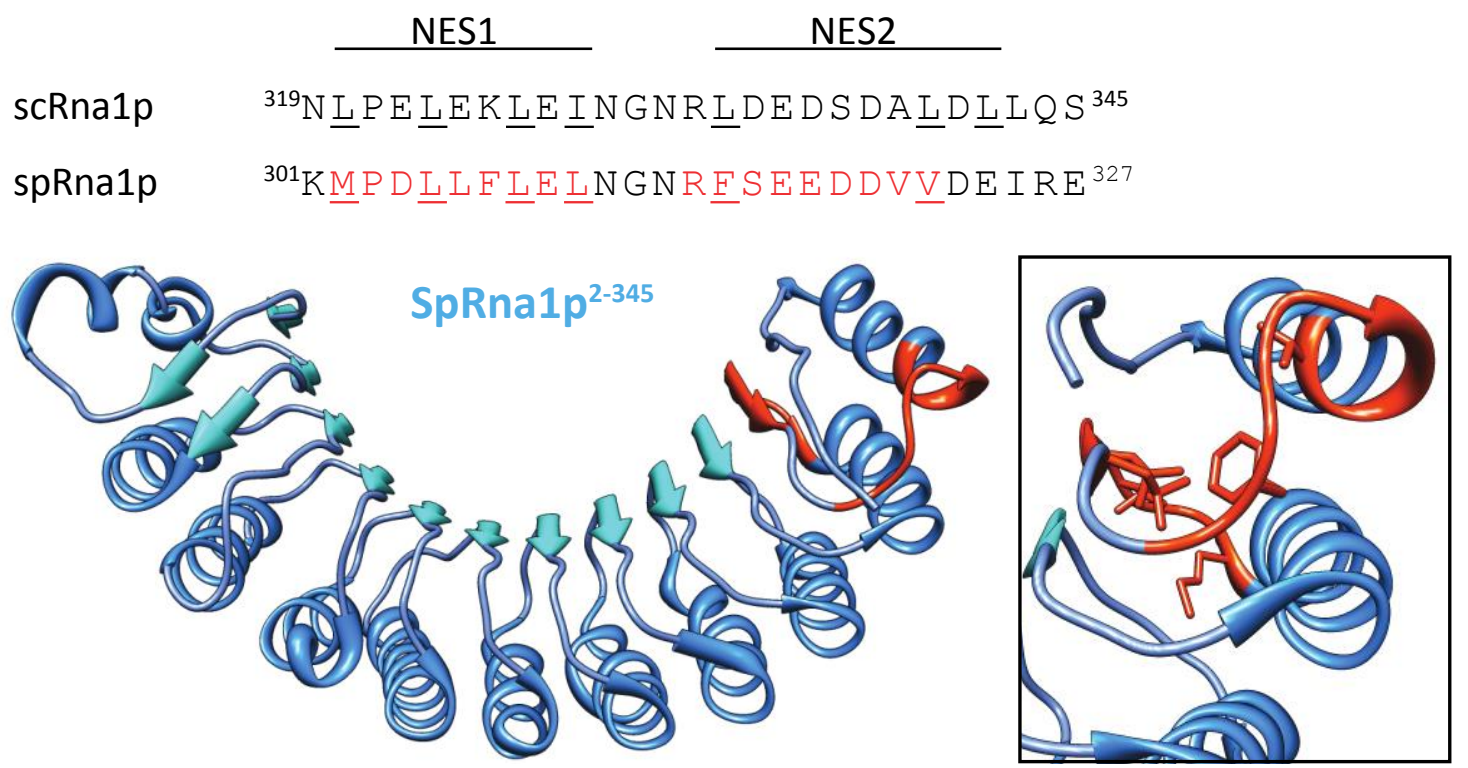

Figure 7-12 spRna1p structure and previously suggested NESs

scRna1p and spRna1p sequences aligned for the region covering previously suggested NESs on scRna1p. On top of the sequences, 2 NESs of scRna1p; NES1 and NES2 are indicated. Corresponding regions are marked orange-red on spRna1p sequence. Residues important for $\mathrm{Crm} 1$ interaction are underlined black on scRna1p sequence. Corresponding residues on spRna1p sequence are underlined orange-red. These orange-red regions are also shown on the spRna1p structure (PDB structure 1K5D Chain C). Side chains for underlined residues are show in orange red.

The two suggested NES regions were part of the crystalized spRna1p. They are located within well folded leucine-rich repeat regions and the hydrophobic residues that would be important for Crm1 interaction are clearly buried in the structure (Figure 7-12). For recognizing such "NES", Crm1 would have to locally unfold its cargo, which is not a very plausible scenario. We therefore 
reasoned that the true NES might have escaped detection and so we used the new prediction algorithm to analyze the primary sequence of spRna1p for alternative NES hits.

\subsubsection{Prediction of spRna1p NES hits}

spRna1p is 386 aa long and the first 340 aa are made up of 8 leucine rich repeats (LRRs). Since NESs can also be rich in leucine residues, it is highly likely to find an NES hit on LRR region of the protein. When the sequence was analyzed with the prediction algorithm, 7 PKI-type and 1 REVtype NES hits were identified. Indeed an NES was predicted for all LRRs except the first one. Since 7 of them were in the previously crystallized LRR domain of the protein, we focused on the last hit. This was the very last 14 residues of the protein and had an unusual alanine residue in its $\Phi_{3}$ position. It had no hydrophobic residue in $\Phi_{0}$ position but the neighboring residues were acidic. In total it had a low NES score, but it was the only hit that had a high disorder propensity (Figure 7-13).

\begin{tabular}{|c|c|c|c|c|c|c|c|}
\hline \multicolumn{8}{|c|}{ RNA1_SCHPO } \\
\hline \multicolumn{3}{|c|}{ Disorder } & Sequence & Start & End & Domain & NES Score \\
\hline B & NES & A & & & & & \\
\hline 1 & 1 & 1 & EIPEALRLLLQALL & 78 & 91 & n.i.d. & 600 \\
\hline 2 & 2 & 2 & AGAKIARALQELAV & 138 & 151 & in LRR & 5600 \\
\hline 1 & 1 & 1 & RPEGIEHLLLEGLAY & 200 & 214 & n.i.d. & 800 \\
\hline 1 & 1 & 1 & EGLAYCQELKVLDL & 210 & 223 & in LRR & 1000 \\
\hline 1 & 1 & 1 & AVVDAFSKLENIGL & 263 & 276 & in LRR & 1800 \\
\hline 1 & 1 & 1 & GLQTLRLQYNEIEL & 275 & 288 & in LRR & 2700 \\
\hline 1 & 1 & 1 & MPDLLFLEL & 302 & 310 & n.i.d. & REV-type \\
\hline 3 & 3 & 3 & EDKELADELSKAHI & 373 & 386 & n.i.d. & 1800 \\
\hline
\end{tabular}

Figure 7-13 NES prediction for spRna1p

Schpo is the Uniprot abbreviation for organism Schizosaccharomyces pombe. From the 3 disorder values (B) represents the 6aa before NES, (NES) represents the predicted hit, and (A) represents the 6aa after NES. 'n.i.d' stands for 'not in any domain'. Hits that reside in the previously crystalized region are shaded gray.

\subsubsection{Validation of spRna1p NES hit}

The first experiment to validate the NES candidate was a C-terminal truncation of spRna1p. Both full-length spRna1p and spRna1p lacking the last 3 amino acids $\left(s p R n a 1 p^{\Delta C 3}\right.$ ) were expressed as His10-ZZ-Tev fusions. The last 3 amino acids covered the $\Phi_{3}$ and $\Phi_{4}$ positions. The candidate NES hit and PKI NES were also expressed with the same tag. We performed binding assays with scCrm1 either in the absence or presence of RanGTP (Figure 7-14). 
A)

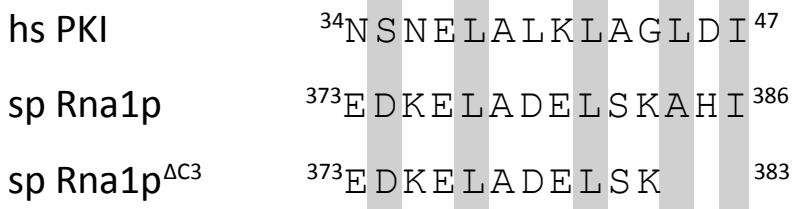

B)

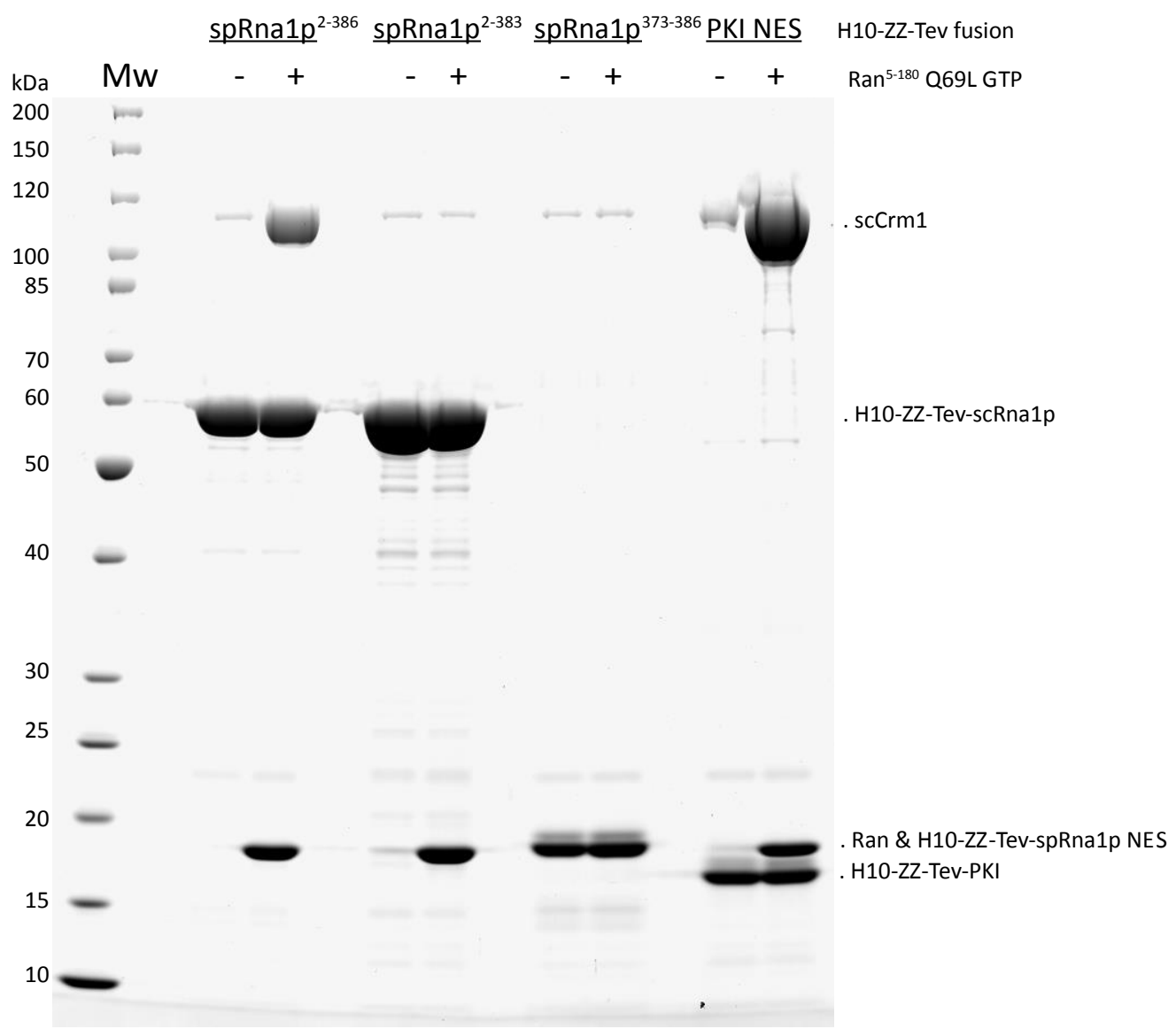

Figure 7-14 RanGTP dependent scCrm1 binding of spRna1p

The reactions were performed in $500 \mu$ volume with $2 \mu \mathrm{M} \mathrm{scCrm1}$ and $2 \mu \mathrm{M}$ H10-ZZ-Tev-NES fusion cargoes. For the reactions with RanGTP, $3 \mu \mathrm{M} \operatorname{Ran}_{5-180}$ Q69L GTP was added. Final buffer concentration was adjusted to $50 \mathrm{mM}$ Tris/HCl $7.5,60 \mathrm{mM} \mathrm{NaCl}, 2 \mathrm{mM} \mathrm{Mg}(\mathrm{OAC})_{2}, 5 \mathrm{mM} \mathrm{DTT}$. After 2 hours of incubation at $4^{\circ} \mathrm{C}$, ZZ-affibody beads were added to pull down the cargo and the bound proteins. Elutions were analyzed with SDS-PAGE.

A) Alignment of PKI NES to spRna1p. Ф residues are indicated above the PKI NES and their alignments with NES hits are shaded gray. Start and end residue numbers of NESs are indicated in the full-length protein context. B) SDS-PAGE analysis of RanGTP dependent Saccharomyces cerevisiae $\mathrm{Crm1}$ (scCrm1) binding of NES hits. $\mathrm{H} 10$ stands for N-terminal 10 histidine residues, Z (in ZZ) stands for IgG-binding domain of the Staphylococcal protein A, and Tev stands for Tobacco Etch Virus protease recognition sequence. Protein ladder is abbreviated with ' $\mathrm{Mw}$ ' for molecular weight, and protein sizes are indicated on the left side of the corresponding bands. Samples without RanGTP are indicated with a ' - ' sign, and samples with RanGTP are indicated with ' + ' sign.

spRna1p shows a significant RanGTP dependent scCrm1 binding. This binding is lost upon removal of last 3 amino acids from spRna1p. Although scCrm1 binding was gone, Ran was still eluted with the spRna1p ${ }^{\Delta C 3}$, due to background binding of Ran to ZZ-affibody beads at low salt conditions. Interestingly, the isolated NES did not show RanGTP-dependent Crm1 binding, suggesting that the functional export signal is bipartite and includes additional parts of the Rna1p molecule. 
To further validate the NES-like sequence within the last 14 residues, we rationally designed point mutants of the full-length protein. If it were the NES, binding strength would respond to the mutations in the $\Phi$ position. We anticipated the following potential outcomes: Mutation of the predicted $\Phi_{4}$ position from isoleucine to serine would weaken while mutating the alanine in $\Phi_{1}$ position to leucine should further strengthen the binding.

The binding assay was done with streptavidin agarose beads, which show less background binding

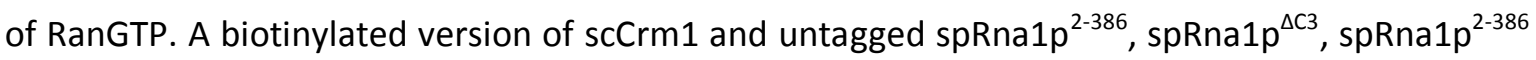
1386S, spRna1 ${ }^{2-386} \mathrm{~A} 384 \mathrm{~L}$ were used for the assay (Figure 7-15). 
A)

hs PKI

$\begin{array}{lllll}\Phi_{0} & \Phi_{1} & \Phi_{2} & \Phi_{3} & \Phi_{4}\end{array}$

${ }^{34} \mathrm{NSNELALKLAGLDI}$

sp Rna1p $\quad{ }^{373}$ E DKELADELSKAH I 386

sp Rna1p ${ }^{\Delta C 3} \quad{ }^{373}$ E DKELADELSK $\quad 383$

sp Rna1p I386S $\quad{ }^{373}$ EDKELADELSKAH S ${ }^{386}$

sp Rna1p A384L ${ }^{373}$ EDKELADELSKLHI I86

B)

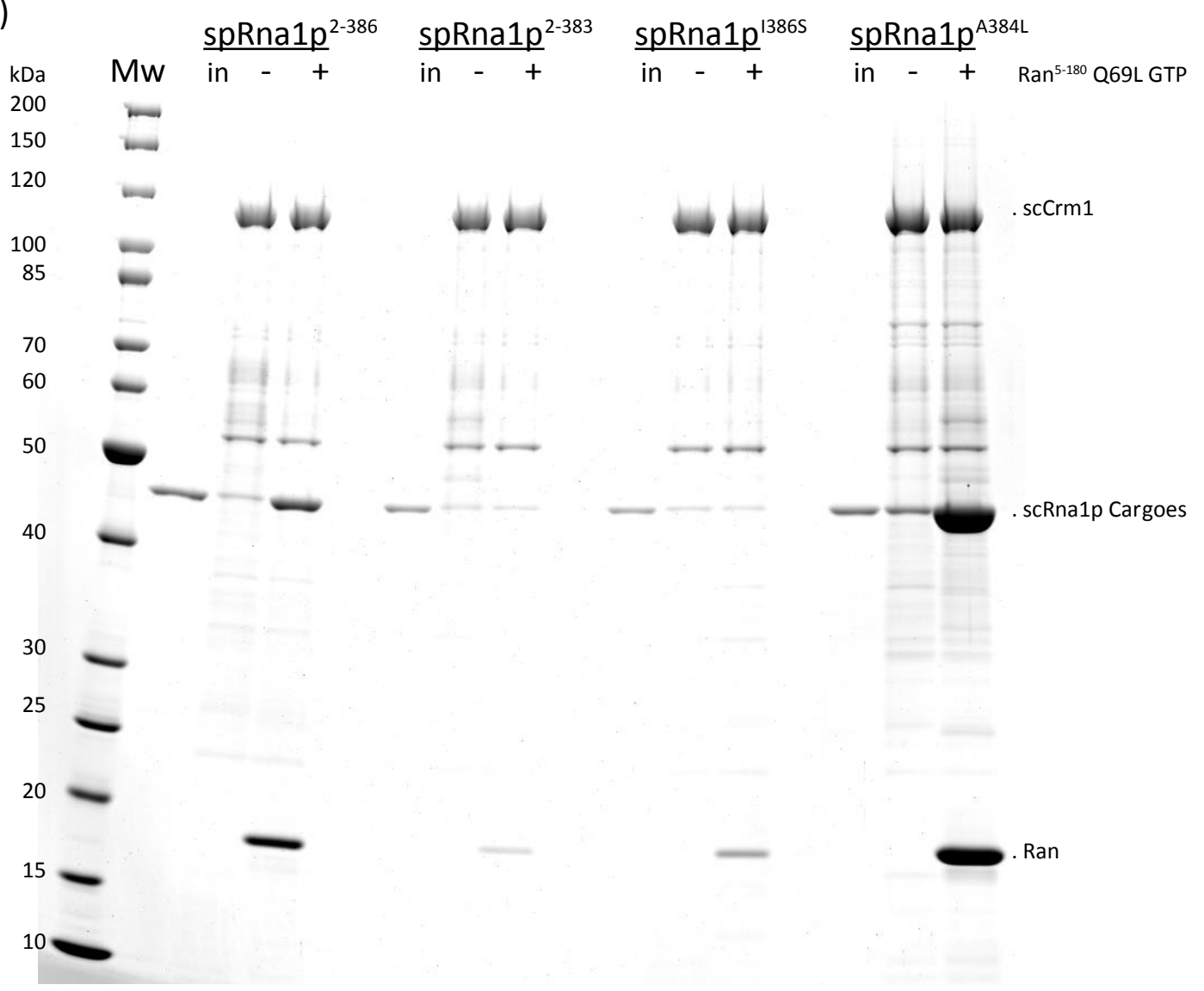

Figure 7-15 RanGTP dependent scCrm1 binding of spRna1p and point mutants

The reactions were performed in $500 \mu$ volume with $2 \mu \mathrm{M}$ biotinylated scCrm1 and $2 \mu \mathrm{M}$ cargo. For the reactions with RanGTP, $3 \mu \mathrm{M} \operatorname{Ran}_{5-180}$ Q69L GTP was added. Final buffer concentration was adjusted to $50 \mathrm{mM}$ Tris/ $\mathrm{HCl} 7.5,60 \mathrm{mM}$ $\mathrm{NaCl}, 2 \mathrm{mM} \mathrm{Mg}(\mathrm{OAc})_{2}, 5 \mathrm{mM} \mathrm{DTT}$. After 2 hours of incubation at $4^{\circ} \mathrm{C}$, streptavidin-agarose beads were added to pull down the cargo and the bound proteins. Elution was done with $37^{\circ} \mathrm{C}$ SDS sample buffer which is enough for elution of cargo and Ran but did not fully disrupt streptavidin-biotin interaction. Elutions were analyzed with SDS-PAGE.

A) Alignment of PKI NES to spRna1p wt and mutants. $\Phi$ residues are indicated above the PKI NES and their alignments with NES hits are shaded gray. Start and end residue numbers of NESs are indicated in the full-length protein context. B) SDS-PAGE analysis of RanGTP dependent SCCRM1 binding of NES hits. H10 stands for N-terminal 10 histidine residues, Z (in ZZ) stands for IgG-binding domain of the Staphylococcal protein A, and Tev stands for Tobacco Etch Virus protease recognition sequence. Protein ladder is abbreviated with ' $\mathrm{Mw}^{\prime}$ ' for molecular weight, and protein sizes are indicated on the left side of the corresponding bands. Samples without RanGTP are indicated with a '-' sign, and samples with RanGTP are indicated with ' + ' sign.

Full-length spRna1p was bound to the beads in the presence of RanGTP, and was not bound when RanGTP was absent. . The previously described mutant lacking the $3 \mathrm{C}$-terminal residues served as an additional control. Interestingly a similar result was obtained upon mutating the $\Phi_{4}$ position 
was mutated from isoleucine to serine. Consistent with this, when the $\Phi_{1}$ was mutated from alanine to leucine the binding was greatly enhanced. These experiments strongly suggest that the NES hit was identified with the correct $\Phi$ spacing.

Testing the localization phenotype of the protein in a cellular context would provide a more stringent test. Therefore the spRna1p NES was also tested by transient transfection of HeLa cell. To test the experimental setup, three PKI NES versions, wt PKI, super PKI (sPKI) with enhanced CRM1 binding, and PKI $\Phi_{4} \mathrm{~A}$ mutant with weaker CRM1 binding, were fused to GFP. NLS and NES of elF2 $\beta$ was fused to mCherry and used as control. Each GFP vector was cotransfected with a mCherry-eIF2 $\beta$ NES, and cells were fixed after $24 \mathrm{~h}$. DAPI was used to stain DNA. wtPKI showed a prominent nuclear exclusion, and the PKI $\Phi_{4} \mathrm{~A}$ mutant lost this exclusion. SPKI was localized to nuclear rim. Since SPKI can bind to CRM1 without RanGTP, disassembly of the export complex is inefficient, and it was stalled at the NPCs. CRM1 was blocked by SPKI, which resulted in mislocalization of the control NES fusion (Figure 7-16).

A)
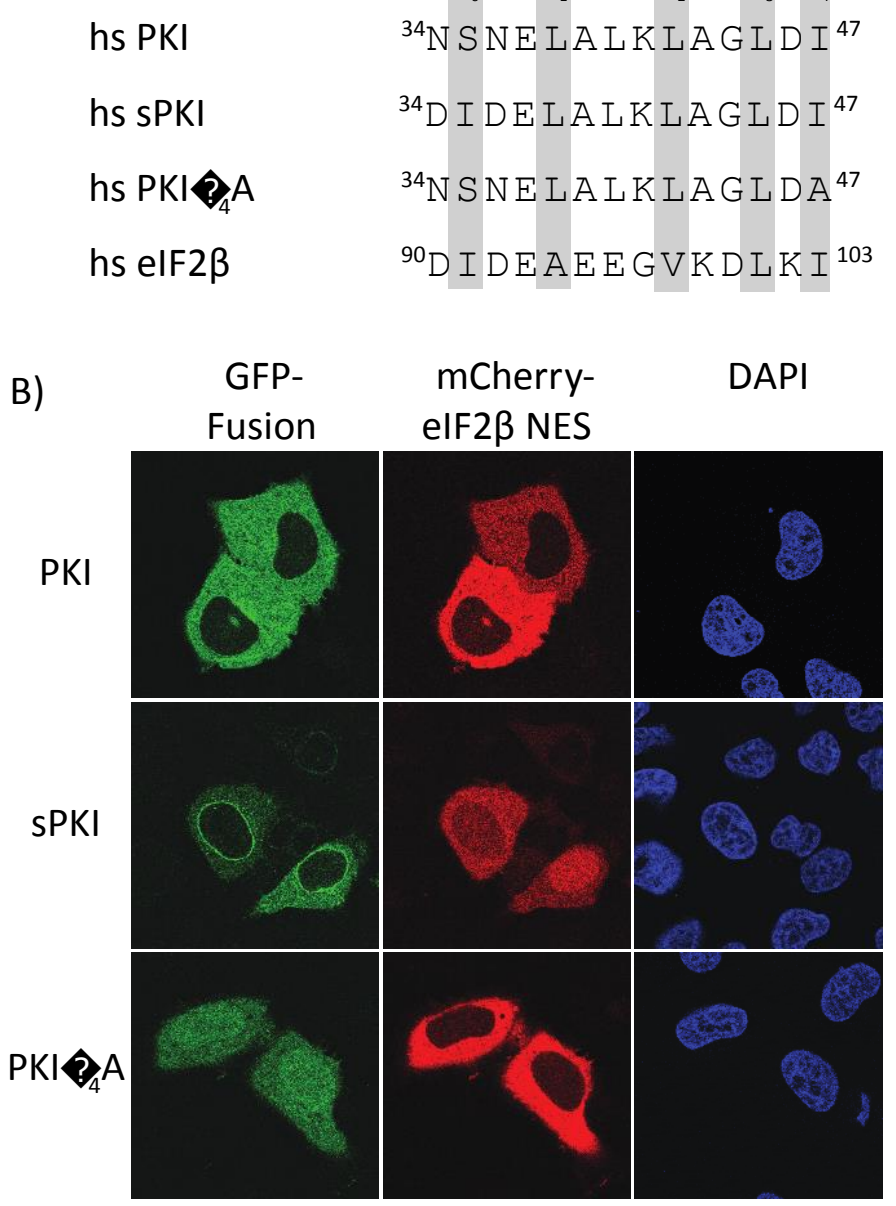

Figure 7-16 Phenotypic outcomes of different GFP-NES fusions

3 versions of PKI were fused to GFP, and cotransfected with the mCherry fusion with elF2 $365-114$, which contains both an NLS and an NES. DAPI staining was used for DNA. 
To test the effect of the NES hit on spRna1p localization, wt and $\triangle$ NES Rna1p were fused to GFP, and cotransfected with NLS and NES of elF2 $\beta$ fused to mCherry. GFP-spRna1p was fully cytoplasmic and deletion of NES on Rna1p resulted in nuclear leakage of the protein. To enhance the effect of NES deletion, an SV40 NLS was fused between GFP and wt Rna1p and Rna1p $\triangle$ NES. GFP-SV40NLS-Rna1p was cytoplasmic, whereas deletion of the $C$ terminal NES resulted in total nuclear accumulation (Figure 7-17).

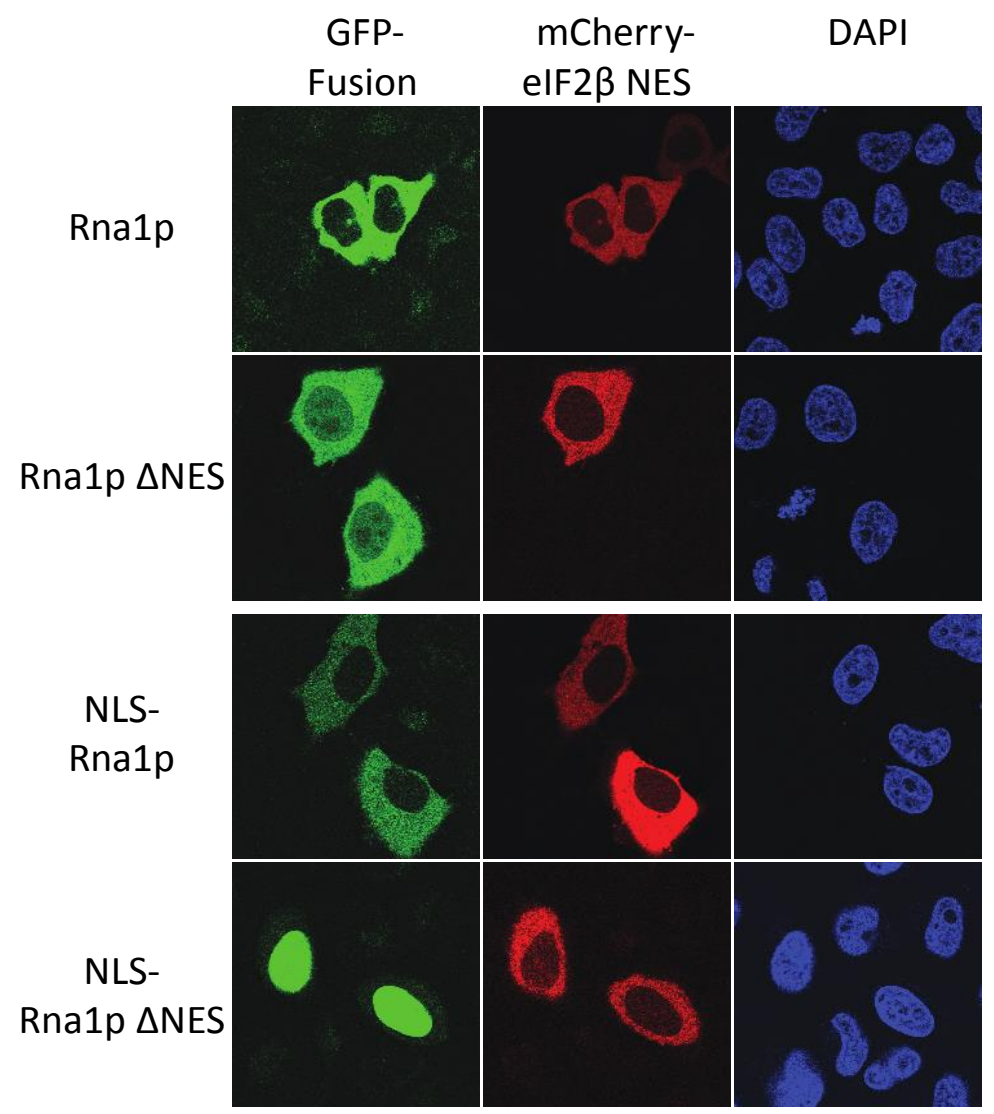

Figure 7-17 Localization of different GFP spRna1p fusions

Full-length spRna1p and $\triangle N E S$ version was fused to GFP or GFP-SV40 NLS, and cotransfected with mCherry fusion of elF2 $\beta 65-114$ that contains both an NLS and an NES. DAPI staining was used for DNA.

The very C-terminal 14 residues stretch is the NES of spRna1p as confirmed by the binding experiments and HeLa transfections. The full-length spRna1p has a stronger binding than the NES peptide itself. This is an indication that rest of the protein, either the LRRs or the poly glutamic acid region, or both contribute to its interaction with $\mathrm{Crm} 1$.

spRna1p was the second protein that was analyzed for an NES with the new prediction algorithm. There was only one hit that was out of the previously crystalized region. It had a low NES score since it had an alanine in its $\Phi_{3}$ position. This NES hit with the low NES score was indeed a poor 
CRM1 binder, but in the context of full-length protein, it was strong enough to sustain a RanGTP dependent binding to and very efficient export by Crm1. 


\subsection{RANGTP DEPENDENT CRM1 BINDERS FROM CYTOSOLIC HELA EXTRACT}

We were further interested in a more complete picture of the RanGTP dependent CRM1 binders. Although it has been known that CRM1 is the most versatile NTR, it was not clear how large the pool of CRM1 exported cargoes actually is. This pool probably comprises not only direct CRM1 binders, but also interaction partners of direct binders. Direct binders may function as adaptors for export of larger complexes. To address these questions, we designed and optimized affinity chromatography on immobilized CRM1. We used extracts from a human cell line as a starting material and mass spectrometry (MS) for identifying candidate cargoes from the eluates.

\subsubsection{CRM1 affinity chromatography of cytoplasmic HeLa extract}

To come up with a complete list of CRM1 exported cargoes, we used the cytoplasmic S10 HeLa extract as the source of RanGTP-dependent CRM1 binders. For a low background binding we optimized the binding conditions and the chromatography system. HeLa extract showed quite high background binding with IgG-sepharose, or anti ZZ affibody-silica beads. Tests on streptavidin-agarose beads gave much better results.

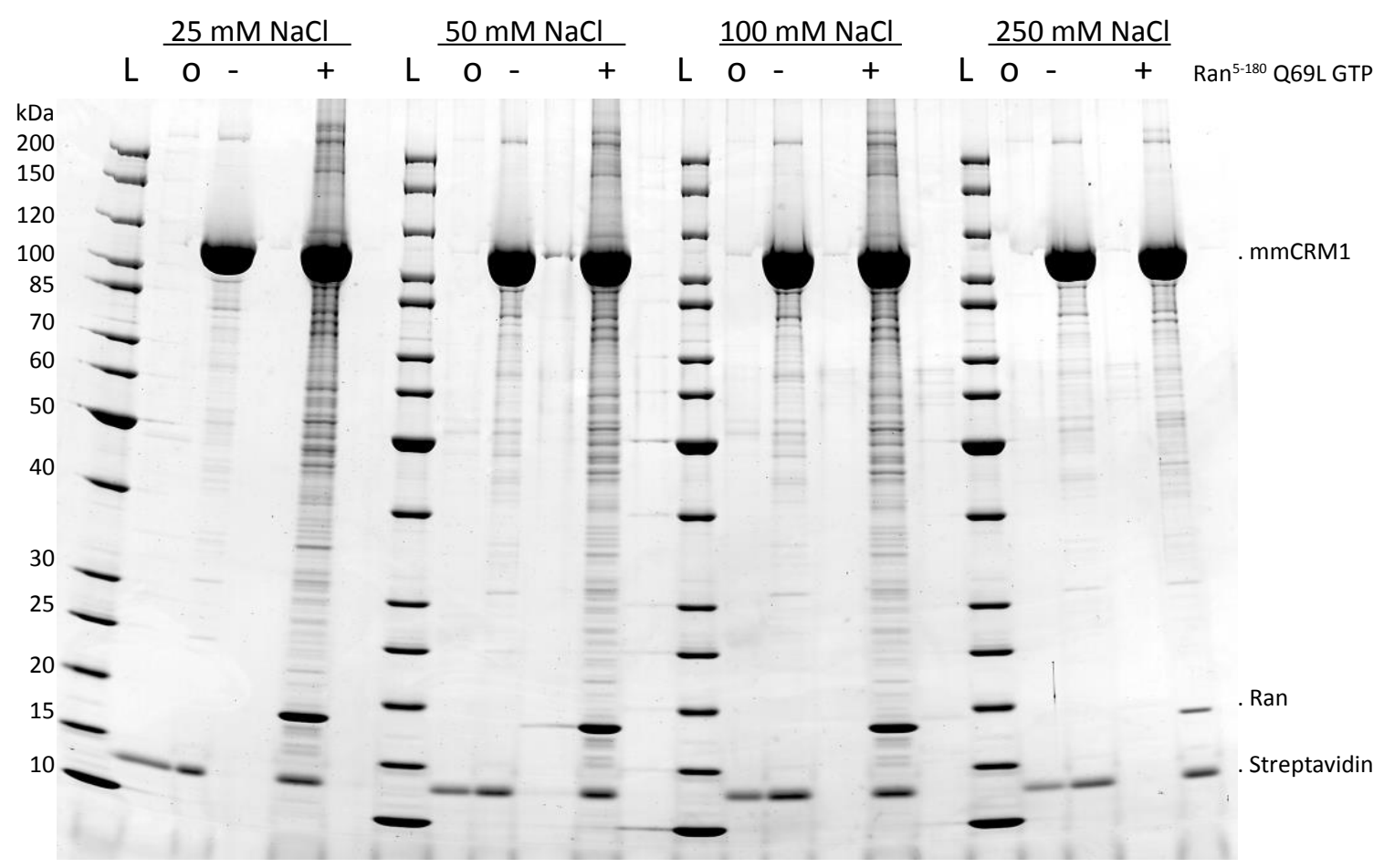

Figure 7-18 Salt sensitivity of RanGTP dependent mmCRM1 interaction of cargoes from HeLa S10 extract.

Affinity chromatography of HeLa S10 extract with Biotin-CRM1 immobilized streptavidin-agarose beads.

For each reaction $0.5 \mathrm{nmol}$ of biotin-CRM1 was immobilized on $20 \mu \mathrm{l}$ of streptavidin-agarose beads, and washed with free biotin. Binding reaction was performed in a final volume of $500 \mu \mathrm{l}$ for $3 \mathrm{~h}$ at $4^{\circ} \mathrm{C}$ with rotation. For each binding experiment $100 \mu$ l of cytoplasmic HeLa S10 extract was used. For samples with RanGTP, $2 \mu$ M Ran ${ }_{5-180}$ Q69L GTP was added. $20 \mu \mathrm{l}$ beads were boiled in $100 \mu \mathrm{l}$ SDS sample buffer to retrieve all bound material.

' $\mathrm{L}$ ' represents the protein ladder, and the corresponding sizes of the ladder bands are indicated on the left side of the first ladder band. Absence or presence of RanGTP is indicated with '-' or ' + ' sign. Background binding of HeLa extract to 
the streptavidin-agarose beads without CRM1 is indicated with 'o' sign. Affinity chromatography was done at different salt concentrations as indicated above the gel.

For CRM1 affinity chromatography, a biotinylated version of mmCRM1 was bacterially expressed and immobilized to the streptavidin-agarose beads. The beads were then washed with free biotin to block remaining biotin binding sites. These beads were used in affinity chromatography of cargos from cytoplasmic HeLa S10 extract either in the absence or presence of RanGTP. To optimize the incubation conditions, a salt screen was performed at 25, 50, 100 and $250 \mathrm{mM} \mathrm{NaCl}$ concentrations.

At $250 \mathrm{mM} \mathrm{NaCl}$ concentration, the RanGTP dependent binding was mostly gone, as also evident from the intensity of the Ran band. RanGTP dependence of the binders was most impressive at low salt concentrations (Figure 7-18).

\subsubsection{CRM1 Affinity Chromatography for SILAC-Based Mass Spectrometry}

The most convenient way of analyzing these global protein pools was mass spectrometry. That's why we further analyzed Ran- and Ran+ lanes with MS to resolve the highly complex pool of proteins in the elutions. CRM1 binding can be a salt sensitive interaction. PKI NES would bind only in the presence of RanGTP when $100 \mathrm{mM} \mathrm{NaCl}$ is used in the binding conditions. As salt concentration goes up, this binding will become weaker. And if salt concentration goes down, a RanGTP independent CRM1 interaction will emerge. That would mean that affinity chromatography at low salt conditions might favor cargo binding also in the absence of RanGTP. Although a very prominent difference was visible between RanGTP+ and RanGTP- lanes at $25 \mathrm{mM}$ $\mathrm{NaCl}$ condition, it still required comparison of protein levels between two samples to clearly identify the RanGTP dependent binders (Figure 7-18). Non-quantitative MS analysis is suited for identification of the proteins in a given sample, however for comparison of protein levels in two different samples, it is not sufficient.

Many different MS methods have been applied for quantification of the protein amounts in the sample (Wilm, 2009). Among them, stable isotope labeling by amino acids in cell culture (SILAC) has proven to be a useful technique in analysis of many proteomics studies (Ong and Mann, 2006). SILAC is a metabolic labeling method where natural amino acids lysine and arginine ('light') are replaced with their ${ }^{2} \mathrm{H},{ }^{13} \mathrm{C}$ and ${ }^{15} \mathrm{~N}$ labeled forms ('heavy') in cell culture. With light and heavy cells I refer to cells grown in the corresponding media. Cytoplasmic extracts prepared from cells grown in either light or heavy medium were kindly provided by Miroslav Nikolov from Mass Spectrometry Research Group, MPI-BPC. 
SILAC based comparison of Ran- and Ran+ samples depends on the assumption that metabolic labeling by heavy amino acids does not change the protein composition of the cell extract. To test this assumption, identical volumes of light and heavy extracts were mixed, and run on SDS-PAGE and the complete lane was analyzed with MS (Figure 7-19).

H/L Distribution

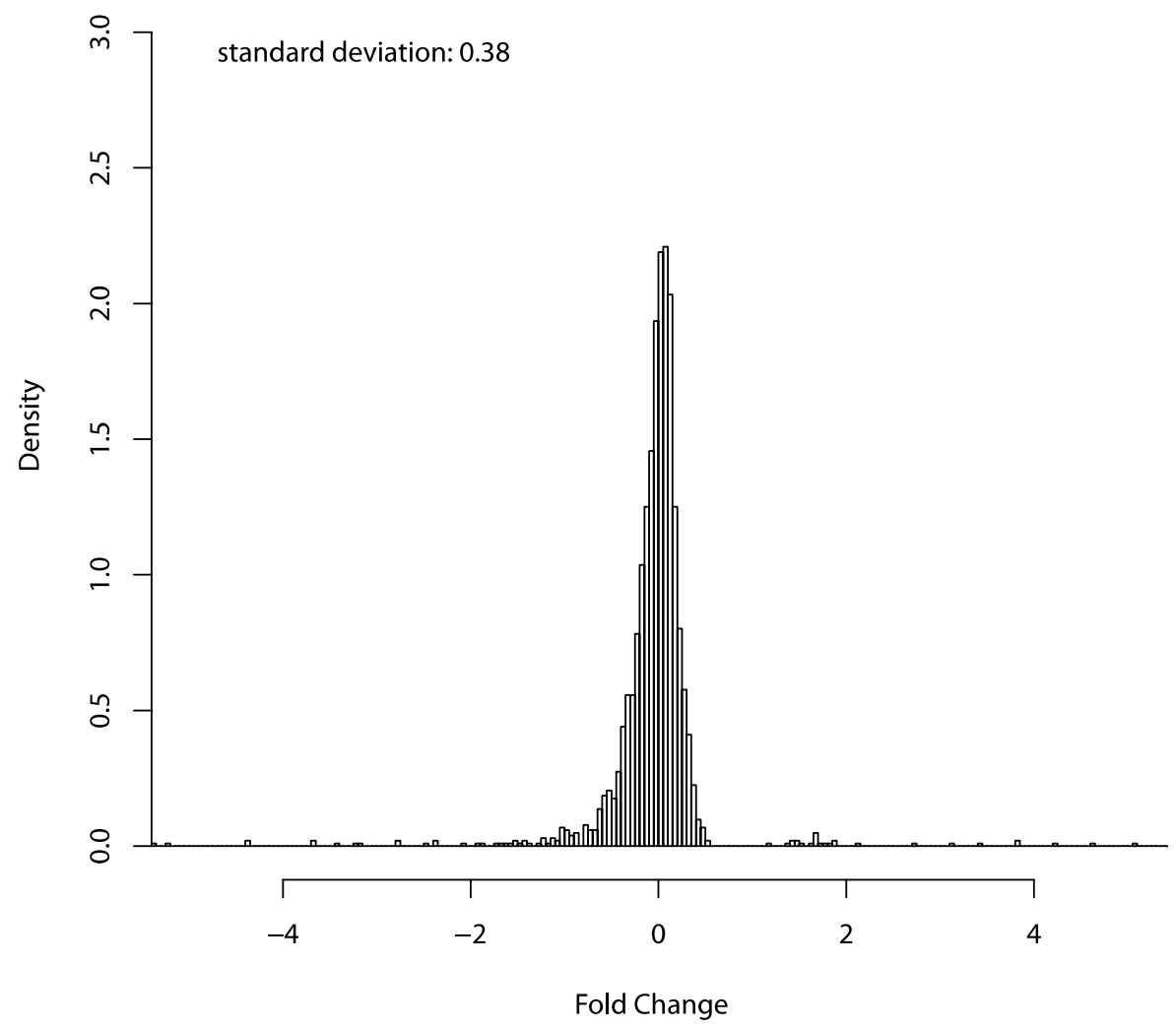

Figure 7-19 Distribution of protein ratios in heavy and light HeLa extracts

SILAC ratios were calculated from a 1 to 1 mixture of heavy and light extracts, and distribution of $\log _{2}$ (Ratios) were subjected to a Gaussian distribution analysis for the standard deviation.

More than 2800 proteins were identified from cytoplasmic HeLa extracts. Although this does not cover whole cytoplasmic proteome, it contained sufficient number of proteins to obtain reliable statistics on the proteins levels. Proteins were present in very similar amount in both heavy and light extracts with a standard deviation of 0.38 for $\log _{2}$ (Heavy/Light) values.

For each reaction $0.5 \mathrm{nmol}$ of biotin-mmCRM1 was immobilized on $20 \mu \mathrm{l}$ of streptavidin-agarose beads, and washed with free biotin. Binding reaction was done in a final volume of $500 \mu \mathrm{l}$ for $3 \mathrm{~h}$ at $4^{\circ} \mathrm{C}$ with rotation. For each binding experiment $100 \mu$ of light or heavy cytoplasmic HeLa extract was used. For samples with RanGTP, $2 \mu \mathrm{M} \operatorname{Ran}_{5-180}$ Q69L GTP was added. Elution was done with $37^{\circ} \mathrm{C}$ SDS sample buffer which is enough for elution of cargoes and Ran but does not fully dissociate biotinylated mmCRM1 from streptavidin, and the most intense band on SDS-PAGE was 
suppressed. Since the total eluate had considerably low CRM1 amounts, it was more representative for the CRM1 binders. This also enables an easier identification on MS for proteins co-migrating with CRM1 band. $10 \mu \mathrm{l}$ of eluate was analyzed on SDS-PAGE (Figure 7-20).

A)

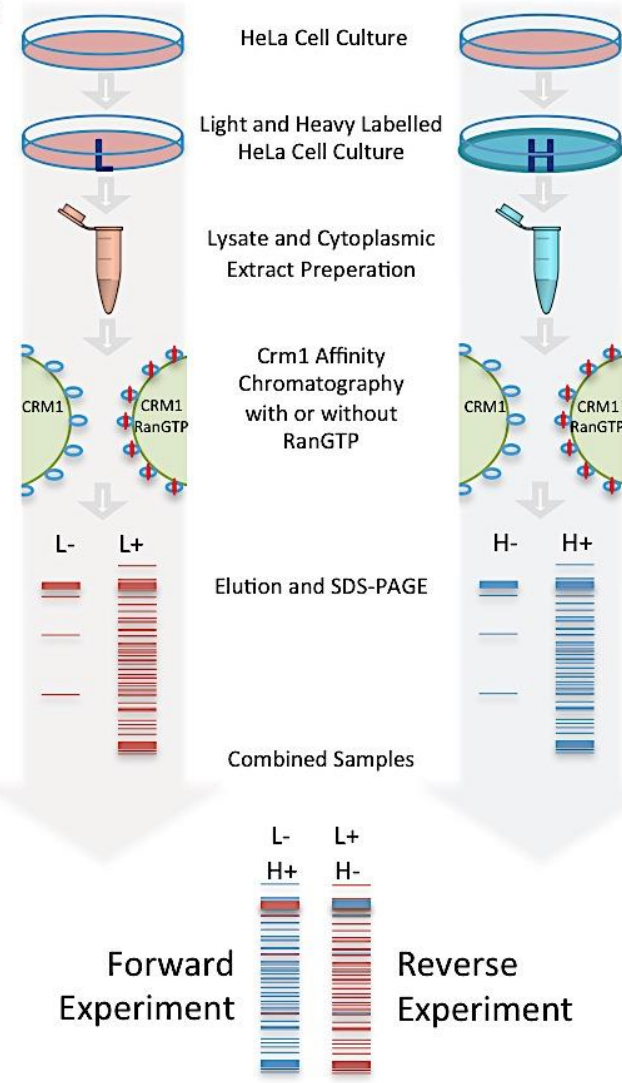

B)

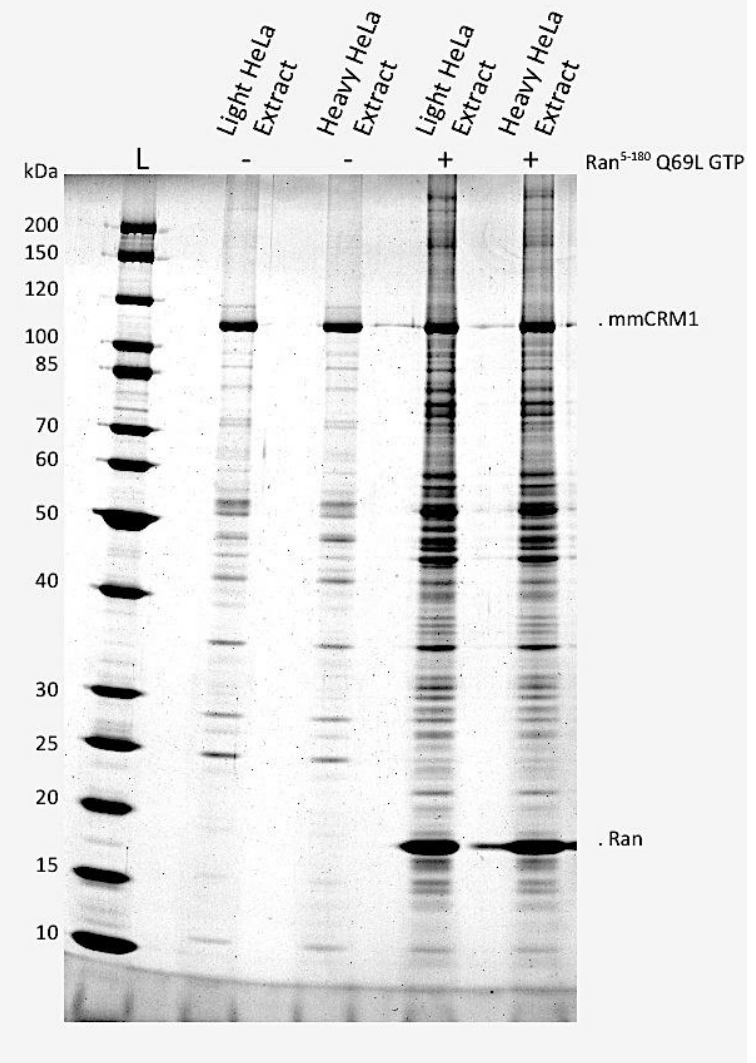

Figure 7-20 CRM1 affinity chromatography of cytoplasmic HeLa extracts produced with SILAC method

A) Schematic depiction of SILAC CRM1 affinity chromatography experiment. B) Affinity chromatography of light and heavy labeled cytoplasmic HeLa S10 extract with Biotin-mmCRM1 immobilized streptavidin-agarose beads. ' $L$ ' represents the protein ladder, and the corresponding sizes of the ladder bands are indicated on the left side of the first ladder band. Absence or presence of RanGTP is indicated with '-' or ' + ' sign. Extracts produced from light and heavy labeled cells are indicated as "Light HeLa Extract" or "Heavy HeLa Extract".

With SILAC based methods, it is possible to analyze a light sample together with a heavy sample at the same time with MS. To compare binding of cargoes in the presence or absence of RanGTP to CRM1, different Ran states of light and heavy elutions are combined. Elution of CRM1 binders from light extract in the absence of RanGTP (L- sample) was mixed with Elution of CRM1 binders from heavy extract in the presence of RanGTP ( $\mathrm{H}+$ sample), and vice versa, Elution of CRM1 binders from heavy extract in the absence of RanGTP ( $\mathrm{H}$ - sample) was mixed with Elution of CRM1 binders from light extract in the presence of RanGTP ( $L+$ sample). The former is called "forward experiment" and the latter is called "reverse experiment". Since MS analysis can distinguish between peptides from heavy and light extracts by residue specific mass difference, in a single analysis $\mathrm{L}$ - sample was compared to $\mathrm{H}+$ sample and also $\mathrm{H}$ - sample was compared to $\mathrm{L}+$ sample. With this experimental setup we not only minimized the error of MS analysis by a streamlined 
processes of samples to be compared, but also repeated the experiment and MS analysis 2 times (forward and reverse experiments). Mass spectrometry analysis was carried out by Samir Karaca from the Mass Spectrometry Research Group of the MPI-BPC.

\subsubsection{Mass Spectrometry Analysis of SILAC CRM1 Affinity Chromatography Eluates}

Forward and reverse experiments were analyzed together. In total there were 3070 proteins with unique Uniprot identifiers. The identified protein levels in Ran+ samples were compared to Ransamples, a ratio value for each protein was obtained, and values were analyzed as $\log _{2}$ values to reflect the fold changes in protein amounts (Figure 7-21).

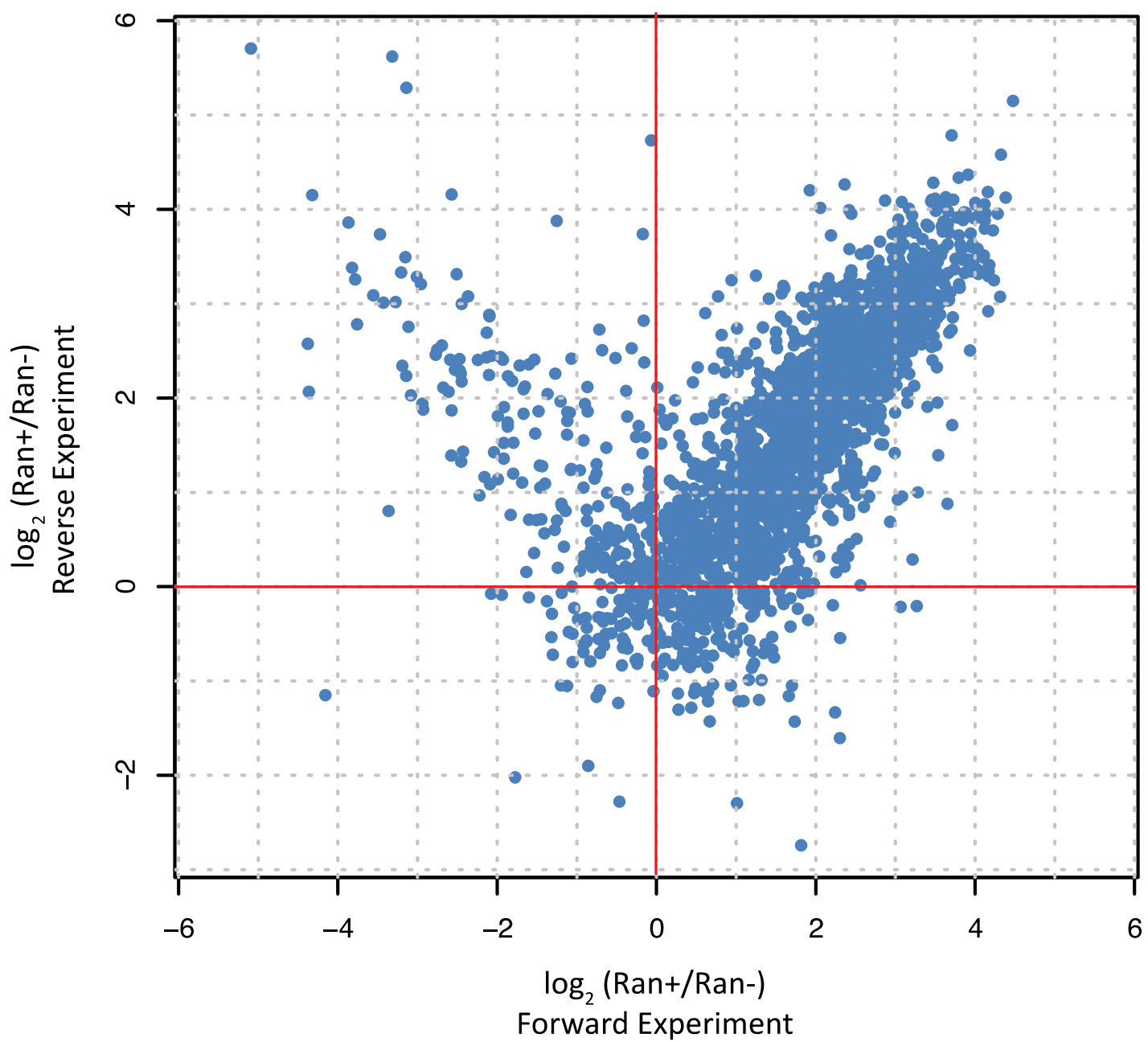

Figure 7-21 SILAC MS analysis of RanGTP dependent CRM1 binders

Ratios of proteins levels obtained by CRM1 affinity chromatography with and without RanGTP were plotted. $\log _{2}$ of values were used to represent fold changes. Ratios from forward experiment were plotted on $\mathrm{x}$-axis and ratios from reverse experiment were plotted on $\mathrm{y}$-axis. $\mathrm{x}=0$ and $\mathrm{y}=0$ lines were shown in red.

The data was divided with two lines that pass through the 0 values of both experiments. If a protein was bound to the beads both with and without RanGTP, the ratio would be 1 , and $\log _{2} 1$ would yield 0. $x$ values higher than 0 show RanGTP dependent binding in forward experiment, and 
y values higher than 0 show RanGTP dependent binding in reverse experiment. The upper left and lower right quartiles show the hits that have different enrichments on mmCRM1 beads in reverse and forward experiments.

The data was divided into further sections. A central circle with formula $x^{2}+y^{2}=2$ contains most of the data points from quartile 1, 3 and 4 . This circle marked the non-specific data points of the analysis that were considered as the background. There were 2 regions that were outside of this circle, one in quartile 1 and one in quartile 2 (Figure 7-22).

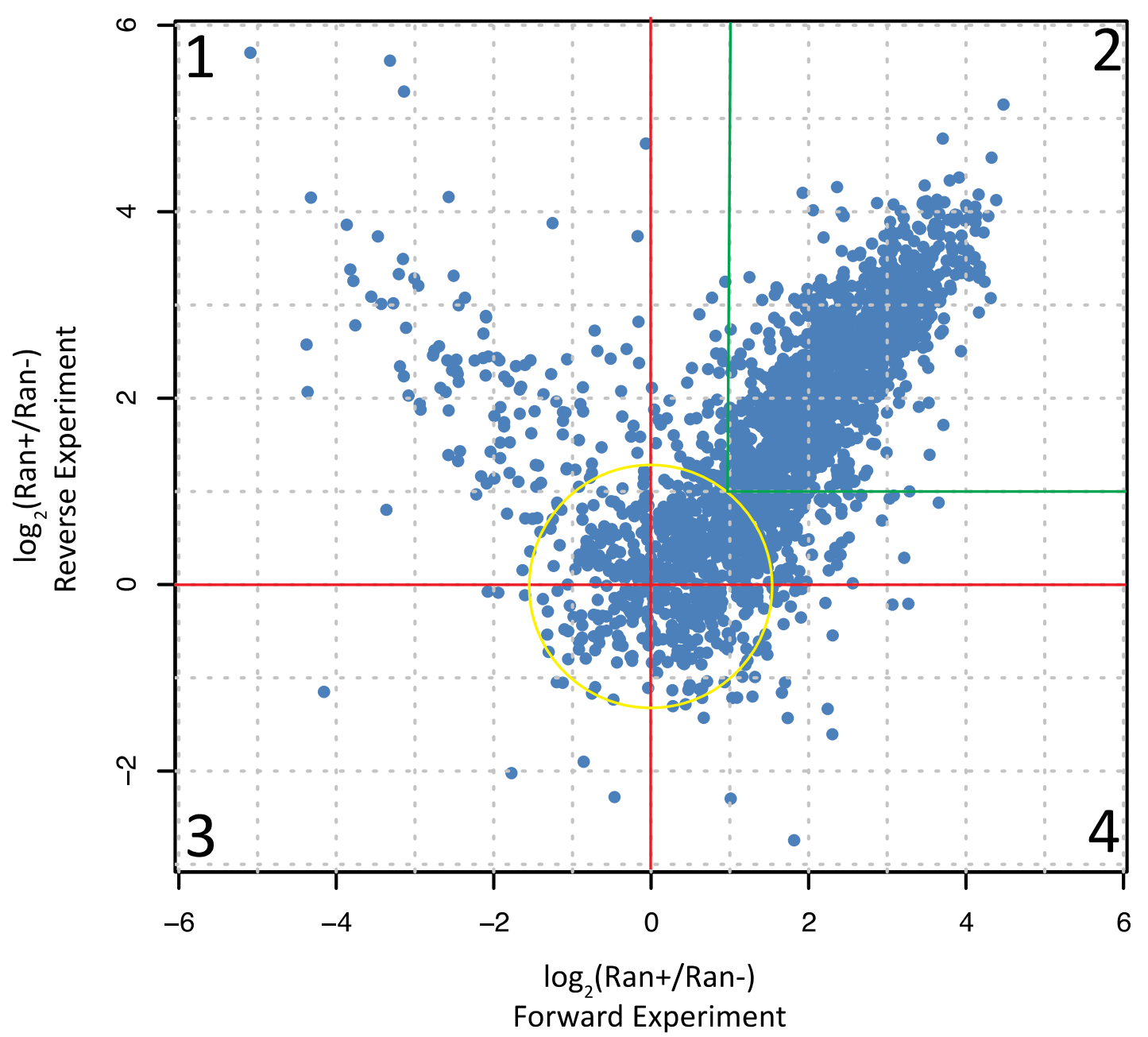

Figure 7-22 SILAC MS data with region markers

Ratios of proteins levels in CRM1 affinity chromatography with and without RanGTP were plotted. $\log _{2}$ of values were used to represent fold changes. Ratios from forward experiment were plotted on $x$-axis and ratios from reverse experiment were plotted on $y$-axis. $x=0$ and $y=0$ lines were shown in red. Each quartile divided by these 2 red lines are numbered 1 to 4 . A central circle with the formula $x_{2}+y_{2}=2$ is drawn in yellow and the region between 2 green lines mark the proteins that show $\mathrm{x}$ and $\mathrm{y}$ values above 1 .

Quartile 1 had data points that were reproduced in both experiments with an inverse correlation.

Every sample of mass spectrometry is contaminated with many proteins from environment; a very common example is keratin. Since every contaminant is devoid of heavy labeled amino acids, 
they contribute to the light sample. In forward experiment, ratios were calculated for heavy Ran+ sample divided by light Ran- sample. Light contaminants only contributed to the denominator, and since their heavy counterpart was missing, it gave a value less than 1 , and $\log _{2}$ value was negative. For the reverse experiment, ratios were calculated for light Ran+ sample divided by heavy Ran- sample. In this case, light contaminants only contributed to the numerator, and since their heavy counterpart was missing, it gave a value more than 1 , and $\log _{2}$ value was positive. Although most of these contaminants are filtered during data processing, there were still some that contributed. Also the proteins Ran and CRM1 were in this part of the data, because they were bacterially expressed and purified without any heavy amino acids. Another group of proteins were the ones that show inconsistent binding in 2 experiments with regards to RanGTP. This part of data was excluded from the analysis (Figure 7-22).

The more interesting portion of the data resided in $2^{\text {nd }}$ quartile, where proteins were enriched on CRM1 beads in a RanGTP dependent manner in both experiments. Proteins that were at least 2 fold enriched in CRM1 affinity chromatography in the presence of RanGTP over CRM1 affinity chromatography in the absence of RanGTP were taken into consideration. The region on graph was marked with 2 green lines that pass through $\log _{2} 2$ values on $x$ and $y$-axis (Figure 7-22). Out of 3070 proteins identified in reverse and forward experiments, 1263 proteins fell in between these two lines. Proteins in this region were not only enriched in Ran+ samples of both experiments. They also gave a very similar result in both experiments, which was visible on the graph since data was scatter along $x=y$ line on quartile $2, x>1$ and $y>1$ region. We considered this region as the promising part of our data.

To compare our data to literature, we used two different sources of previously described NES cargoes. First one was the NESdb; curated database of CRM1 cargoes from various species (Xu et al., 2012a). Second one was a previous SILAC study based on changes in nuclear and cytoplasmic protein pools of HeLa cells upon Leptomycin B treatment (referred as LMB study) (Thakar et al., 2013).

NESdb combines the previously published data for proteins that are exported by CRM1. Two protein lists were compiled from NESdb. First one was composed of human proteins of the NESdb. There were 120 proteins in this first list. Second list was composed of human proteins that had an ortholog from Canis familiaris (dog), Mus musculus (mouse), Rattus norvegicus (rat), Gallus gallus (chicken), Xenopus laevis (African clawed frog) and Anaxyrus americanus (American toad), which was described as CRM1 cargoes in the NESdb. The second list contained 54 proteins. 
In total there were 174 proteins from NESdb. 59 out these proteins were among proteins identified in our SILAC MS analysis, and 50 of them had $\log _{2}$ ratios greater than 1 in both forward and reverse experiment (Figure 7-23).
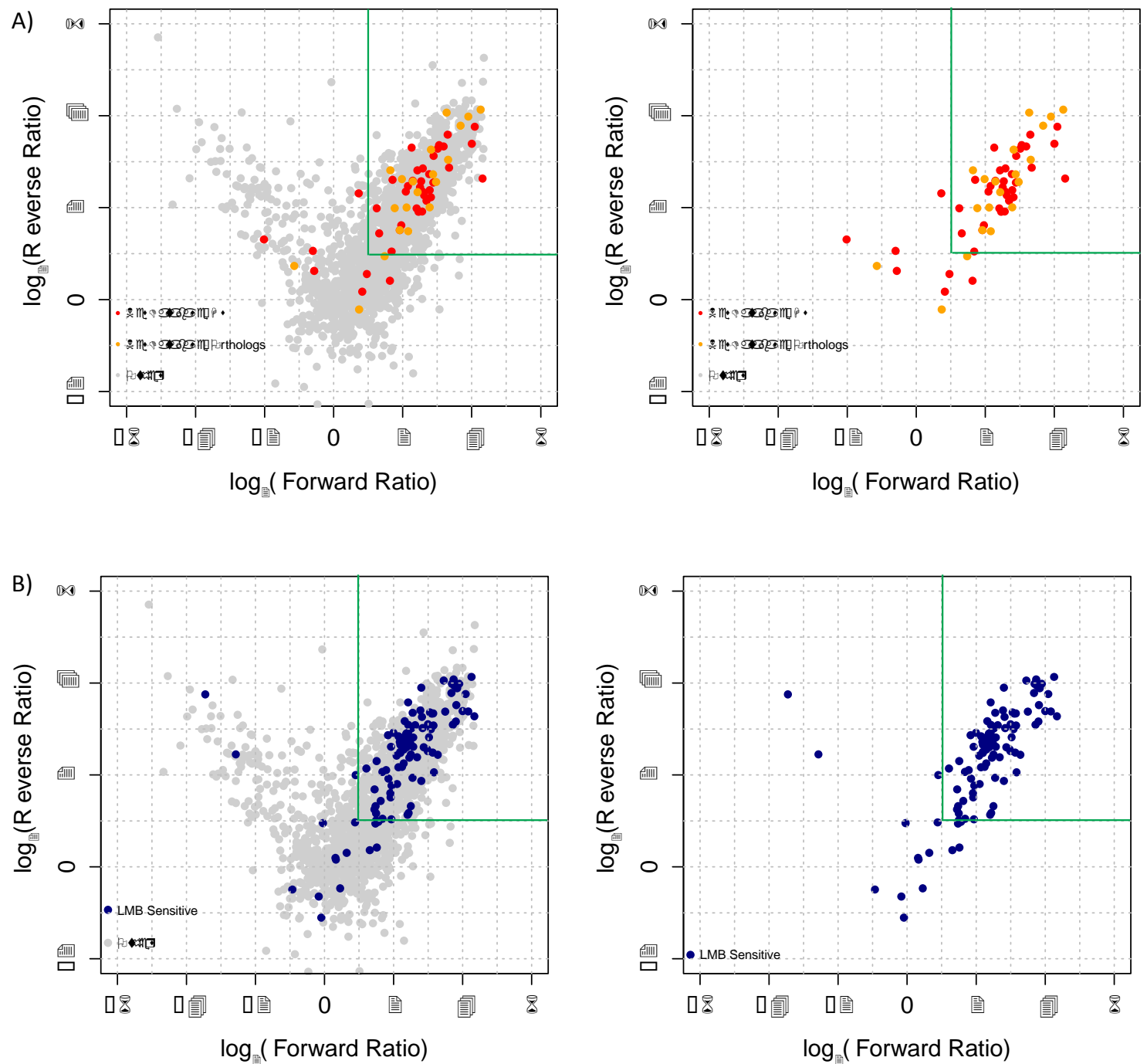

Figure 7-23 NESdb and LMB Sensitive Hits on SILAC MS data

A) Human proteins from NESdb (red) and human ortholog of proteins from NESdb (orange) are marked on our data set. B) Proteins from LMB were marked blue on our data set.

We also compared our data to the previous LMB study. Leptomycin B treatment specifically inhibits CRM1 export activity by covalently modifying a cysteine residue in the CRM1 hydrophobic pocket. LMB study identified 138 proteins that either showed cytoplasmic depletion or nuclear enrichment upon Leptomycin B treatment. Out of these 138 proteins 104 were in our data set, and 90 of them had a $\log _{2}$ ratio greater than 1 in both forward and reverse experiments. Both comparisons showed that previously annotated CRM1 cargoes were enriched in our data. 
We further analyzed the prominent part of our data for over- and under-represented protein groups. We used KEGG Pathways, KEGG Brite and Gene Ontology databases to compile primary protein groupings (Ashburner et al., 2000; Kanehisa, 2013). We then hand curated these data to come up protein groups, which are over- or under-represented in the specifically CRM1/ RanGTPbound fraction (Figure 7-24).

A) Common protein groups in MS data set

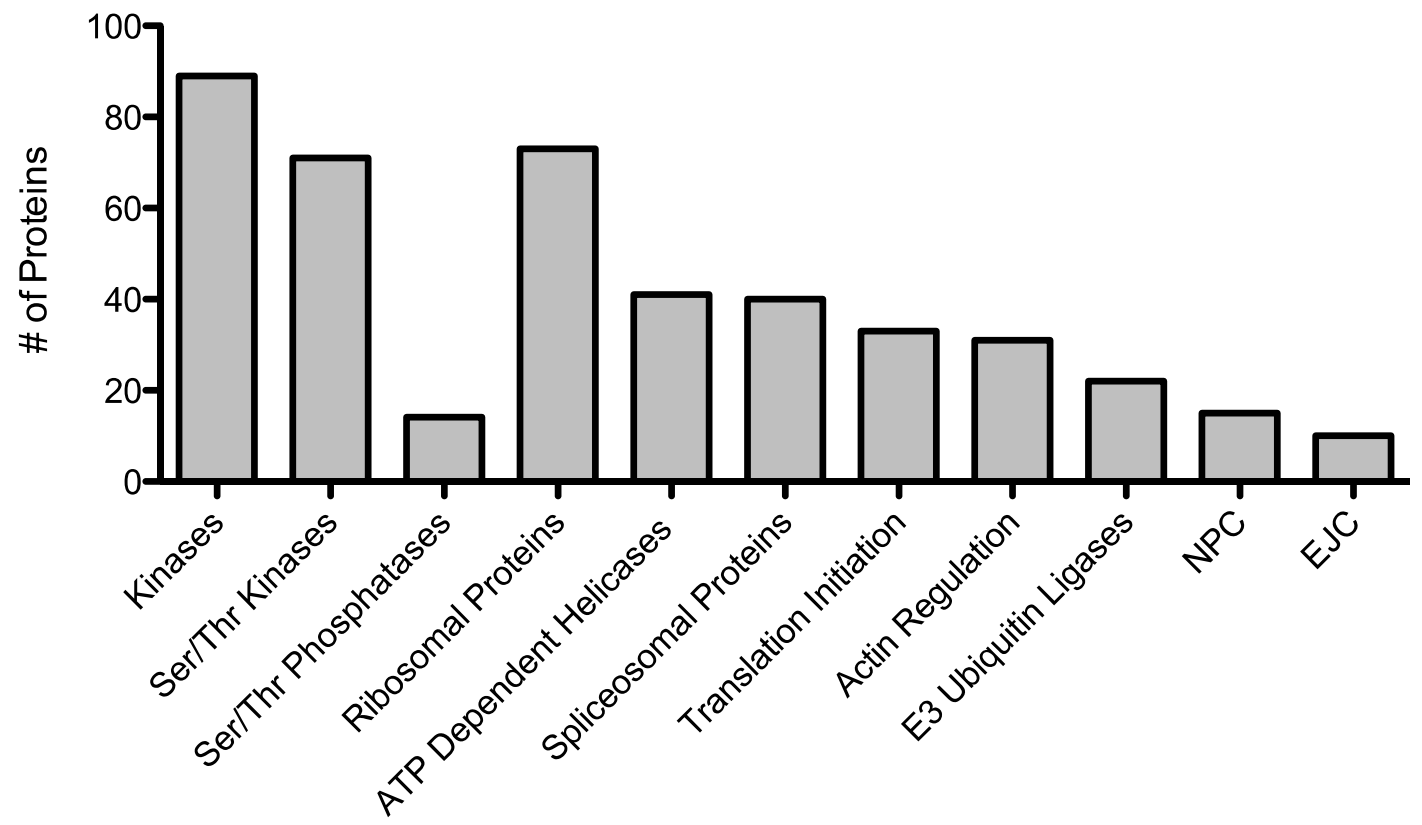

B) Underrepresented protein groups

C) Prominent NES hits

Metabolic Pathways

Glycolysis-Gluconeogenesis

Amino Acid Metabolism

Fatty Acid Metabolism

TCA

...

Cell Adhesion

Phagosome

Proteasome

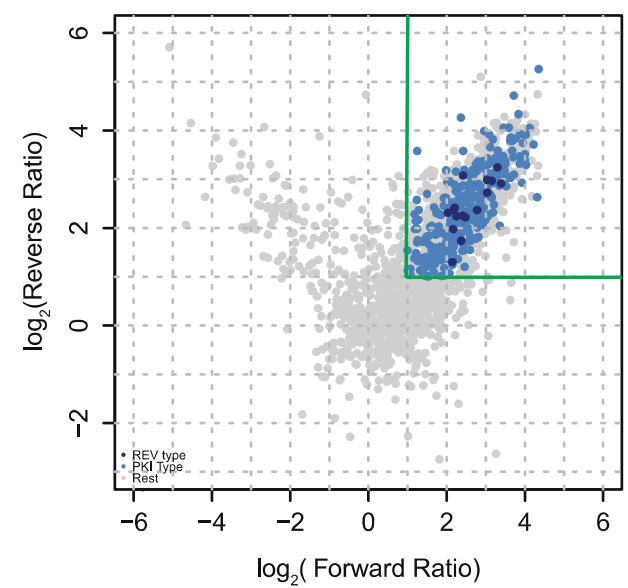

Figure 7-24 Protein groups that are over or under represented in MS data set

A) Number of proteins in MS data from the indicated groups. B) Pathways and activities that are underrepresented in the MS data. C) Prominent PKI-type and REV-type NES predictions in the MS data.

Most of the ribosomal proteins are found in our dataset. Besides them, we find serine threonine kinases, ATP dependent helicases, spliceosomal proteins, translation initiation factors, actin 
regulators, and E3 ubiquitin ligases. We do not find proteins of metabolic pathways, cell adhesion, phagosome, and proteasome. The protein groups are not limited by these listed here.

We analyzed the prominent portion of our data for existence of NESs with our prediction algorithms. Out of 1263 proteins, 321 were predicted to have a PKI-type or REV-type NES. 16 of these proteins were predicted to have a REV-type NES with an aggregated disorder propensity higher than 1. 309 proteins were predicted to have a PKI-type NES with an NES score higher than 5000 and an aggregated disorder propensity higher than 1. 4 proteins were predicted to have both types. We found an NES in $25 \%$ of the MS data set. To assess its significance, we compared this to NES prediction in a CRM1 binder depleted pool of proteins. To construct this pool, we subtracted all the proteins found in the CRM1 chromatography from the proteins found in the total cytosolic extract. Out of 471 such proteins, 44 were predicted to have an NES with the same constraints. Only $9.3 \%$ of the CRM1 binder depleted pool of proteins was predicted to have a significant NES hit.

First of the most complete set of protein groups was the ribosomal proteins. Eukaryotic ribosomes are composed RNA-protein complexes, have 2 main subunits, $60 \mathrm{~S}$ and 40S. $60 \mathrm{~S}$ has 46 proteins and 40S has 46 proteins in the core structure (Ben-Shem et al., 2011). 42 proteins of 60S ribosomal subunit, and 31 proteins of $40 \mathrm{~S}$ ribosomal subunit were present in our data. Since ribosomal subunits are assembled in nucleus, they need to be transported to the cytoplasm. It has been shown that subunits are transported separately in a CRM1 dependent manner. (Thomas and Kutay, 2003). 60S subunit is exported via an adapter protein, NMD3 (Trotta et al., 2003). NMD3 was part of our data set. The CRM1 dependent export mechanism of $40 \mathrm{~S}$ subunit is not elucidated so far.

A second group of proteins were translation factors. In our data set we found 38 proteins from many initiation factors (Table 7-3). We also found 4 proteins from elongation factors, and 2 proteins from release factors (Table 7-4). We found all core initiation complexes including full members of core initiation complexes elF2, elF2B, elF3. All initiation core complexes had at least one member with a predicted NES hit. elF2 $\beta$ NES was identified in this study, and it is the member of elF2 complex that binds to CRM1. We identified the NES on elF2 $\beta$, and the other members of elF2 complex were not predicted to have an NES hit. 


\begin{tabular}{|c|c|c|}
\hline Complex & Protein Name & NES Prediction \\
\hline \multicolumn{3}{|c|}{ Core initiation factors } \\
\hline & elF1 & \\
\hline & elF1A & \\
\hline elF2 & elF2 alpha & \\
\hline elF2 & elF2 beta & $*$ \\
\hline elF2 & elF2 gamma & \\
\hline elF2B & elF2B alpha & \\
\hline elF2B & elF2B beta & \\
\hline elF2B & elF2B gamma & \\
\hline elF2B & elF2B delta & \\
\hline elF2B & elF2B epsilon & $*$ \\
\hline elF3 & elF3a & $*$ \\
\hline elF3 & elF3b & \\
\hline elF3 & elF3c & $*$ \\
\hline elF3 & elF3d & \\
\hline elF3 & elF3e & \\
\hline elF3 & elF3f & \\
\hline elF3 & elF3g & $*$ \\
\hline elF3 & elF3h & \\
\hline elF3 & elF3i & \\
\hline elF3 & elF3j & \\
\hline elF3 & elF3k & \\
\hline elF3 & elF3l & \\
\hline \multirow[t]{8}{*}{ elF3 } & elF3m & \\
\hline & elF4A-1 & $*$ \\
\hline & elF4E & \\
\hline & elF4G-1 & \\
\hline & elF4B & $*$ \\
\hline & elF4H & \\
\hline & elF5 & \\
\hline & elF5B & $*$ \\
\hline \multicolumn{3}{|c|}{ Other initiation factors } \\
\hline & elF2A & $*$ \\
\hline & elF2D & $*$ \\
\hline & elF4E type 2 & \\
\hline & elF6 & \\
\hline & elF4G-2 (p97) & \\
\hline & elF4G-3 & \\
\hline & PABP & \\
\hline & DHX29 & $*$ \\
\hline
\end{tabular}

Table 7-3 List of translation initiation factors from MS data

Core initiation factors are grouped according to classification by (Trotta et al., 2003). A significant NES hit by the prediction algorithm is indicated by ' ${ }^{\prime \prime}$. 


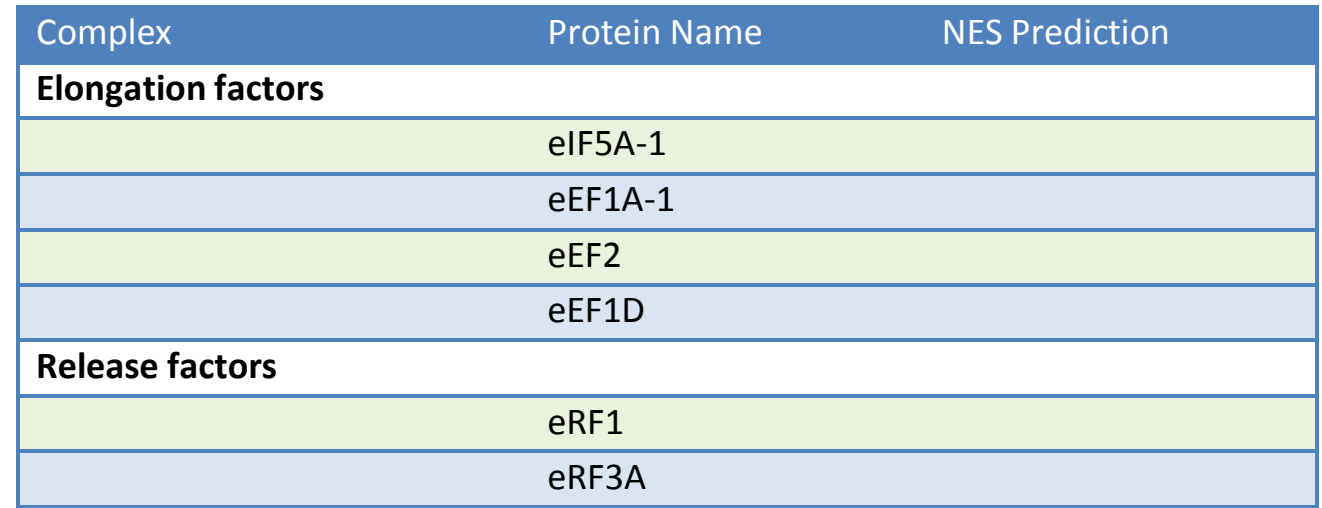

Table 7-4 List of translation elongation and release factors

Taken together, we were able to come up with a protein pool that was highly representative of known CRM1 cargoes and also contains many new candidate proteins and protein complexes. Some of these groups make perfect sense for nuclear exclusion, e.g., ribosomal subunits and translation factors, and some that needs further investigation to come up with the biological reasoning for their nuclear exclusion or nucleocytoplasmic shuttling. 


\section{DISCUSSION}

Many routes in the cell have adapted cellular trafficking guided by linear localization sequences. Nucleocytoplasmic trafficking employs such sequences both for import and export. CRM1 is the NTR with the highest workload, responsible for recognition of NESs on many proteins of different functions and families.

\subsection{A NEW PREDICTION ALGORITHM FOR CRM1 DEPENDENT NESS}

Here we presented a new method of predicting CRM1 dependent nuclear export signals. Although this is not the first prediction algorithm for NESs, it has major differences to the previous NES prediction tools. So far all NES prediction tools focused on the pool of known NES sequences, and tried to come up with a consensus to cover them all. The methods to define this consensus ranged from basic alignments to neural networks. All follow the basic principle of fitting hydrophobic residues $L, M, V, F$, and I into a consensus that was deduced from the analysis of a NES pool. This approach is limited by a couple of pitfalls.

First, not all NESs found in the literature are true NESs. Since the very first definition of NES was mainly made up of leucine residues (Bogerd et al., 1996), scientific community was biased while analyzing their proteins of interest for the existence of NESs. Many studies analyzed peptide sequences that fit into an NES consensus in an isolated context, performing binding assays with 10-15 residue long peptides. Although these binding assays can function, it is a poor diagnostic tool for assessment of the functional NES on the full-length protein. Since NESs have a high frequency of hydrophobic residues, there is a high chance for a candidate NES that functions in isolated context, to be buried in the protein structure. An important example of such analysis was done for actin. Actin has two NES like sequences on its primary structure, and these two sequences can direct a reporter protein to cytoplasm when fused to it (Wada et al., 1998). However, the crystal structure of actin (PDB ID: 1ATN-A, Kabsch et al., 1990) clearly shows that critical hydrophobic residues of these two "NESs" are deeply buried in the actin structure and thus inaccessible for CRM1 binding. Indeed, it was later shown that full-length actin does not bind to CRM1, but is exported by the dedicated NTR Exportin 6 (Stuven et al., 2003). Likewise, actin is clearly excluded from the CRM1-dependent exportome analyzed in this thesis.

Further NES misannotation originates from sub-optimally designed studies using Leptomycin B (LMB). Leptomycin B fits into the hydrophobic pocket of CRM1 and covalently modifies a cysteine residue, thus blocks NES binding to this pocket (Kudo et al., 1999a). The immediate effect of LMB is blocking of cargo export, but in an experimental setting it is not easy to identify the effect. 
When LMB blocks CRM1, expected phenotype of a CRM1 cargo is entry into the nucleus. For small proteins this can be fast since the NPCs do not block passive diffusion of small proteins. Also shuttling proteins that have both NLS and NES accumulate in the nucleus when CRM1 is inhibited. But for some proteins it may take a very long time for them to diffuse into the nucleus, although they possess an NES. These proteins will fail in the LMB test although they are CRM1 cargoes. On the other hand, some proteins will show LMB sensitive localization, although they are not CRM1 cargoes. Prolonged incubations with LMB have secondary effects since CRM1 is responsible for exclusion of RanGAP and RanBP1, which mediate the RanGTP gradient. Also changes in localization of true CRM1 cargoes will have secondary effects, and will change localization of nonCRM1 cargo proteins. Since previous prediction tools first prepared a library of previously published CRM1 dependent NESs, they included false hits, and based their consensus on these mixed pool of true and false NES sequences.

Second, not all sequence features can be attributed to the NES function. The first NES prediction algorithm NetNES (la Cour et al., 2004), trained a hidden Markov model with a true and a false set of NES sequences. At the end they came up with an NES Scoring based on the primary sequence of the query. This approach looks at all the different features with a single constraint. In fact it is possible to extract different features from the NES sequence and grade them separately. One obvious feature is the disorder tendency. For accessibility of the NES by CRM1, it should be kept solvent exposed, unless there are other mechanisms involved in conformational changes of cargoes. A previous NES prediction tool, NESsential (Fu et al., 2011), considered disorder as a NES feature. Although they improved the precision of the NES prediction, there is more to extract from NES and disorder prediction. NESsential focuses on the disorder prediction for residues covering the NES region. $\mathrm{N}$-terminus of PKI-type NESs is $\alpha$-helical and thus creates a local dip in the disorder tendencies. To point out this feature, we considered disorder propensities of 3 regions, 6 amino acids before the NES hit, the NES hit itself, and 6 amino acids after the NES hit. The local dip in the disorder propensity of linear motifs features has been previously described (Fuxreiter et al., 2007).

The history of NES consensus started with a very limited selection of residues that were allowed for the $\Phi$ positions. This initially was the result of the limited number of know NESs, and when the leucine rich NES consensus was defined, this created a bias towards leucine residues in $\Phi$ positions (Bogerd et al., 1996). As number of identified cargoes increased over time, this definition also got broader to allow L, V, M, F and I in the $\Phi$ positions (la Cour et al., 2004). When known NES sequences were aligned, only the most frequent amino acids made it to a statistical significance. In fact, this was a step where important information was lost due to averaging. 
Previously, others groups described supra-physiological NESs, sequences that can bind to CRM1 with a very high affinity, even in the absence of RanGTP (Engelsma et al., 2004). These supraphysiological binding should be achieved by fulfilling all the requirements for an NES extremely well. Features that contribute to NES-CRM1 interaction (e.g. residues in each $\Phi$ position, Nterminal $\alpha$-helical propensity) can be pushed further to strengthen this interaction (Güttler et al., 2010). When PKI NES is modified to have a stronger affinity for CRM1, it failed to dissociate from CRM1 and localized to the nuclear rim. At higher concentrations it even blocked nuclear exclusion of cherry fused to a positive control NES (Figure 7-16). Functional NESs are kept at a sub-optimal CRM1 affinity for a RanGTP regulated CRM1 binding.

Our prediction algorithm expanded the allowed sequences for $\Phi$ residues based on a systematic mutation study of these positions (Güttler et al., 2010). Although some of these residues are not optimal for the position, we assumed that optimal residues in other positions could compensate for it. To represent this in our algorithm, we established an incremental scoring system. This allowed us to have a very flexible consensus sequence, but also a high precision with the scoring system. Since the algorithm did not depend on previously discovered NESs, it was not influenced by the false positives in the NES databases.

We observed the immediate outcome of this flexible consensus and scoring system on two different proteins, spRna1p and hselF2 $\beta$. Both proteins were known to interact with CRM1 but the responsible NES was not identified. In both cases and unusual alanine residue was in one of the $\Phi$ positions, and was overlooked by previous prediction algorithms. Xu et al. defined a new consensus by analyzing their curated NES database NESdb (Xu et al., 2012b). This analysis revealed a broader consensus, but rare events were still excluded by the consensus NES definition. In their analysis alanine was found more frequently in $\Phi_{1}$ and $\Phi_{2}$ positions then in $\Phi_{3}$ and $\Phi_{4}$ positions. Therefore alanine was restricted to the $\Phi_{1}$ and $\Phi_{2}$ residues. That consensus fails to find the NES on spRna1p, which has an alanine in its $\Phi_{3}$ position. We verified this alanine to be a $\Phi$ pocket residue by mutating the position to leucine and enhancing the interaction.

It is evident from previous studies that acidic residues contribute to the CRM1 NES interaction (Güttler et al., 2010). Eukaryotic Linear Motifs Server (ELM) uses an NES consensus that seeks at least one acidic residue both at the $\mathrm{N}$ and C-terminus of NES hit (Dinkel et al., 2012). This consensus misses any NES that starts with a $\Phi_{1}$ position at the extreme $\mathrm{N}$-terminus or ends with a $\Phi_{4}$ position at extreme $\mathrm{C}$-terminus of the protein sequence. An example would be the spRna1p NES that is at the very end of the protein sequence. Recent crystal structures of CRM1 with bound NES shed light on the contribution of negative residues in N-terminus of the NES. Upstream of the 
CRM1 hydrophobic cleft where $\Phi_{0}$ fits has a positively charged surrounding, and can be involved in electrostatic interactions with negatively charged residues on an NES (Figure 7-3).

One NES that we identified but cannot explain by our NES consensus is the NES from Xenopus laevis homolog of elF2 $\beta$ (Figure 7-10). This NES has a threonine residue in its $\Phi_{3}$ position. It might well be that threonine is allowed in this sequence context, but not in PKI NES sequence context. This remains to be tested. An alternative explanation could also be possible based on another mutation screen on super PKI, a modified version of PKI with supra-physiological binding affinity (Güttler et al., 2010). Mutants of super PKI, that had one of the $\Phi$ positions mutated to alanine, were still able to bind to CRM1. It might mean that when NES features are optimal with $4 \Phi$ positions, the other one can be dispensable. Since the mutation study was done with alanine only, we do not know the allowed sequence space for such a trade off.

Another advancement that came with the NES-CRM1 crystal structures was a new $\Phi$ position. This additional $\Phi$ position was preceding the already defined $\Phi_{1-4}$ positions, and the position was named $\Phi_{0}$. This position is not necessary for the interaction, but can significantly influence the binding strength. To evaluate the effect of the $\Phi_{0}$ position and surrounding negative charges, we excluded them from the strict consensus, allowing them to be optional, and gave extra score when they were present.

Crystal structure of REV NES with CRM1 revealed an unusual hydrophobic cleft fitting preference that we analyzed separately from the PKI-type NESs. Definition of REV-type included a strict $\Phi_{0}$, $\Phi_{2}, \Phi_{3}$ and $\Phi_{4}$ positions with common hydrophobic residues, and $\Phi_{1}$, position with a proline residue.

Evaluation of our PKI-type NES prediction algorithm gave very promising results; the combination of NES score with disorder propensity revealed the true NES for the 11 known cargoes (Table 7-1). PKI-type definition covers many previously identified NES sequences and is in accordance with the previous NES consensus definitions. However, evaluation of REV-type NES matched only 7 NESs from NESdb. REV-type definition is more restrictive than the PKI-type and also was previously not recognized. Still, it is very important since it constitutes another class of NESs and cannot be covered by PKI-type definition. The first NES sequence that was described for Saccharomyces cerevisiae Rna1p is also REV-type NES (Figure 7-12). REV-type NES class is important for an NES definition with very high, if not complete coverage of all NESs. 


\subsection{MASS SPECTROMETRY ANALYSIS OF HELA CRM1 CARGOES}

Having a powerful NES prediction tool in hand, we wanted to analyze a larger pool of proteins to discover novel NES harboring proteins and also new transport trends in the cell. The number of previously known CRM1 cargoes was already more than 100 . To come up with an exhaustive list of proteins, we used a SILAC based quantitative mass spectrometry approach that can compare thousands of proteins from two different pools.

We were not the first ones to employ such a technique. A previous study made use of the CRM1 inhibitor Leptomycin B (LMB) to analyze pool of CRM1 binders (Thakar et al., 2013). They analyzed total pools of nucleus and cytoplasm before and after $3 \mathrm{~h}$ of LMB treatment, to come up with proteins that change localization in response to CRM1 inhibition. There are couples of expected outcomes of this experiment. CRM1 cargoes might diffuse into the nucleus in the absence of functional CRM1, and outcome would be nuclear enrichment of such proteins. It is also possible to see cytoplasmic depletion of shuttling proteins with high turnover rate, since they will be trapped in the nucleus in the absence of CRM1, and the cytoplasmic pool would be degraded. In this study Thakar et al. identified 84 proteins that show cytoplasmic depletion, and 59 proteins that show nuclear accumulation. 5 proteins were in both groups. Data set contained many ribosomal proteins of 605 ribosomal subunit and 15 previously described cargoes.

This experimental system has some limitations in representing all CRM1 dependent export cargoes. CRM1 is responsible for establishment of RanGTP mediated transport system by keeping RanBP1 and RanGAP cytoplasmic. Upon LMB treatment RanBP1 becomes mainly nuclear in 30 min (Plafker and Macara, 2000a). This alone would create problems not only for CRM1 export but also for all RanGTP dependent export complexes. This effect is evident also in the data set that shows nuclear accumulation of NTRs importin $\alpha$, importin 4, importin 8, transportin, and CRM1. The scope of other secondary effects of LMB was not addressed in the paper. Another drawback is the limited passive diffusion. Many potential CRM1 target may be part of larger cytosolic complexes, and thus have a very limited passive diffusion in the absence of CRM1. This experimental approach possesses another problem for identification of low abundant proteins. By analyzing whole cytoplasmic and nuclear fractions, the total complexity of the samples is kept very high and this complexity can mask identification of low abundant proteins or minor changes in protein localizations.

We used RanGTP dependent CRM1 affinity chromatography to enrich CRM1 binders from cytoplasmic HeLa extract. This way we were able to confine the protein pool complexity to CRM1 associated proteins. We supplied enough CRM1 molecules to limit competition, and to enrich 
even low abundant proteins on our CRM1 streptavidin agarose beads in the presence of RanGTP. One of the key experimental advantages of using streptavidin agarose matrix was the significantly low background binding to HeLa cytoplasmic proteins. This low background made it possible to observe the drastic difference between CRM1 binders in the absence and presence of RanGTP. Previous groups also used such binding assays to identify import or export cargoes. Since the significant changes were limited to number of bands on SDS-PAGE, these bands were cut and analyzed by mass spectrometry (Mingot et al., 2001). Our experimental results gave a higher complexity that wasn't possible to analyze on the level of distinct bands, thus we performed whole lane analysis. To be able to compare eluates of CRM1 affinity chromatography in the presence and absence of RanGTP, we used a SILAC based approach. This allowed us to process these two lanes at the same time and compare them with a single analysis. The binding experiment and the mass spectrometry analysis were repeated with the forward and reverse experiments.

Our experimental setup and analysis may also have some drawbacks. (i) Although we enriched for the RanGTP dependent CRM1 binders, we cannot rule out the possibility of losing very low abundant proteins. (ii) Many human proteins are either not expressed in HeLa cells or kept predominantly nuclear and did not exist in our cytoplasmic extracts. (iii) Some proteins might not yield any ionizable peptide by trypsin digestion. (iv) Some proteins might need modifications for functional NESs. (v) CRM1 chromatography was performed at low salt concentrations, and some proteins with very high CRM1 affinity might bind also in the absence of RanGTP, and fail to enrich. Snurportin 1 was an example of these proteins. Snurportin 1 has very strong CRM1 affinity, was bound also in the absence of RanGTP. (vi) Some NESs might be masked in cytoplasmic complexes.

We identified 1263 proteins that were at least two times enriched in the presence of RanGTP in both reverse and forward experiment. Many of the previously known CRM1 cargoes were part of our data. It also had a quite good coverage of the results from the LMB study.

The most prominent protein group in the data set is the ribosomal proteins with 72 hits. They are synthesized in the cytoplasm and then imported into the nucleus. In nucleoli they assemble with ribosomal RNAs into 405 and 605 ribosomal subunits. Nuclear export of these subunits was shown to depend on CRM1 (Thomas and Kutay, 2003). 60S subunit is exported via an adapter protein, NMD3 (Trotta et al., 2003). We find 42 proteins of 605 ribosomal subunit, 31 proteins of 405 ribosomal subunit, and also NMD3 in our data set. So far no vertebrate adapter for 40 subunit was described. A shuttling protein Ltv1 that binds both Crm1 and 40S was described as the adapter in yeast (Seiser et al., 2006). Our data set contains the human orthologs of this protein, 
called protein LTV1 homolog. An NES was identified for yeast Ltv1, but it is highly unlikely to be the true NES, since it has an aspartic acid in $\Phi_{4}$ position. Our prediction algorithm suggests a very C-terminal NES both for yeast and human proteins.

Another prominent group of proteins is the translation initiation factors. Separation of translation and transcription requires strict confinement of the key regulators, translation factors. Translation initiation factors elF2, elF2B, elF3, elF4A-1, elF5 and elF5B, elongation factors eEF1A, eEF1B, and eEF2, and termination factor eRF1 are kept strictly cytoplasmic (Bohnsack et al., 2002). In our data set we find 38 proteins from many initiation factors, 3 proteins from elongation factors, and 2 proteins from release factors. We found and validated the NES on elF2 $\beta$, which can account for the 3 subunits of elF2 complex in our data set. Another prominent initiation factor is the elF3 complex. We found all of 13 members in our data set. elF3G, elF3C and elF3A has one prominent NES hits each, and might be responsible for nuclear exclusion of the complex. elF4A-1, eIF2A,elF2B epsilon, eIF2D, eIF4B, and elF5B are the other members of translation initiation complexes with a significant NES hit. We didn't find any significant hits in release and elongation factors. It needs further validation to see which translation initiation complexes are true CRM1 binders.

21 of 71 serine threonine protein kinases in our data set were predicted to have an NES. Two of them, dual specificity mitogen-activated protein kinase kinase 1 (MP2K1) and mitogen-activated protein kinase-activated protein kinase 2 (MAPK2), were previously shown to have NESs (Fukuda et al., 1996; Engel et al., 1998). MAPK2 is localized to the nucleus and upon stress induced phosphorylation; the NES is activated and exported to the cytoplasm. Since serine threonine protein kinases are involved in highly regulated processes like cell proliferation, programmed cell death, cell differentiation and embryonic development, their localization can also be part of their regulation as in the case of MAPK2. This may mean that some serine threonine protein kinases have a regulated NES, which might require other features than we assumed, and cannot be identified with the prediction algorithm. Also the experimental setup may fail to identify the NESs that require further modifications to become functional. Therefore analysis of NESs in regulated proteins requires much more attention.

There are many other groups of proteins that are part of our mass spectrometric data set, and need further classification into meaningful units, such as soluble complexes. Examples of such soluble complexes in our data are signal recognition particle (SRP), the human Augmin complex (HAUS), Ski complex, Arp2/3 complex and minichromosome maintenance protein complex (MCM). 


\section{OUTLOOK}

The new prediction algorithm can come up with a fine selection of NES candidates, based on the Ф position specific scoring and disorder filtering. This prediction algorithm was based on NES crystal structures and mutation screen of PKI NES $\Phi$ positions. It is possible that a more complete mutation analysis of both PKI and REV-type NESs might reveal other aspects of the consensus definitions, and enlarge the repertoire of allowed amino acids in $\Phi$ positions. Inter-repeat sequences also play a significant role in stability of NES structure. N-terminus of PKI-type NESs has an $\alpha$-helical structure, and amino acids with higher $\alpha$-helical propensities may be preferred at this positions. Also effect of neighboring residues is not fully analyzed. It is known that negatively charged residues are preferred, however there might be other constraints on the allowed amino acids. We want to explore these preferences with a comprehensive NES mutation screen.

Our mass spectrometry data contains exhaustive number of proteins. We so far categorized the proteins into functional groups, but a more comprehensive categorization into soluble protein complexes is needed. With such categorization we will start investigation each soluble complex for the CRM1 binding member with our prediction algorithm. This way we can come up with a comprehensive list of NES. Protein groups without a predicted NES hit also constitute an interesting group of proteins; there might be some CRM1 cargoes with unusual NESs, or even different CRM1 interaction features. One example is translation release factor eRF1, which was shown to be Leptomycin B sensitive (Bohnsack et al., 2002) and also was in our MS data set. There is no NES predicted for eRF1, and it remains to be seen what mechanism behind its leptomycin B sensitivity is.

Since our protocol for identification of CRM1 binders from a complex pool was highly efficient, we want to apply these experimental settings to different protein pools. By comparing CRM1 binder pools of synchronized cells from different stages, we might be able to find CRM1 dependent cell cycle regulators. This application can also performed in other model organism systems. Yeast is a very good candidate since there are already established SILAC protocols and proteomic localization studies. 


\section{MATERIAL AND METHODS}

All described standard methods were performed on the basis of (Sambrook and Russell, 2001).

\subsection{INSTRUMENTS}

\begin{tabular}{|ll|}
\hline Instrument & Manufacturer \\
\hline Sonifier 450 & Branson, UK \\
\hline Eppendorfbiophotometer & Eppendorf \\
\hline Incubator/Climo-Shaker ISF1-X & Kuhner Shaker \\
\hline TCS SP5 microscope & Leica, Mannheim \\
\hline Thermo NanoDrop2000C & peqLab, Germany \\
\hline Äkta-Purifier, Äkta-Explorer & Pharmacia, Upsala, Sweden \\
\hline SensoQuest lab cycler & SensoQuest, Göttingen \\
\hline miniDAWN ${ }^{\text {TM }}$ TREOS ${ }^{\circledR}$ & WyattTechnology, Dernbach \\
\hline DynaPro NanoStar ${ }^{\text {TM }}$ & WyattTechnology, Dernbach \\
\hline 1260 Infinity Quaternary LC System & Agilent Technologies, Waldbronn \\
\hline Shodex ${ }^{\circledR}$ RI-101 & Showa Denko K.K.,Minato-ku, Japan \\
\hline GenePulser & BioRad, Burlington, USA \\
\hline Perfection V700 Photo Scanner & Epson \\
\hline arium ${ }^{\circledR}$ pro UV & Sartorius, Gottingen \\
\hline SB3 rotator & Bibby Scientific, France \\
\hline RVC 2-18 Rotational Vacuum Concentrator & \\
\hline
\end{tabular}

Table 10-1 Laboratory Equipment

\begin{tabular}{|lll|}
\hline Centrifuge & Rotor/Type & Manufacturer \\
\hline Refrigerated tabletop centrifuge & $5415 \mathrm{R}$ & Eppendorf \\
\hline Tabletop centrifuge & 5424 & Eppendorf \\
\hline RC6 plus centrifuge & F9, F10 & Sorvall \\
\hline WX Ultra centrifuge & T647.5, T125.0, Type 45 Ti & Sorvall \\
\hline Discovery M120 & S55A, S45A & Sorvall \\
\hline
\end{tabular}

Table 10-2 Centrifuges and Rotors 


\subsection{PREPARATION OF DNA CONSTRUCTS}

All coding sequences are either acquired from previous lab plasmids, or amplified with specific primers from human cDNA library or yeast genomic DNA.

\begin{tabular}{|ll|}
\hline Coding Sequence & Source \\
\hline mmCRM1 & pTGA021 \\
\hline scCRM1 & pSF879 \\
\hline spRna1p & pDG0044 \\
\hline hsRan 5 -180 Q69L & pKG031 \\
\hline hselF2 $\beta$ & pTGA404 \\
\hline
\end{tabular}

Table 10-3 Sources of Coding Sequences

For preparing DNA constructs, PCR was performed with designed primers and then the product and the target vector were digested with compatible restriction enzymes with different flanking sequences on $5^{\prime}$ and $3^{\prime}$ to allow directional insertion. Digested products were checked for correct size on agarose gel electrophoresis, cut from the gel and purified. Vector and insert were ligated and transformed in electrocompetent E.coli cells. After preparation, all coding regions were sequenced with primers that anneal before and after the region of interest (Seqlab, Göttingen). All of these standard molecular biology methods were performed on the basis of (Sambrook and Russell, 2001). In the following pages, I tried to explain methods that are modified in detail.

\subsubsection{Primer Design}

Primers were designed for sub-cloning, introducing deletions or mutations to the DNA constructs. DNASTAR Lasergene SeqBuilder ${ }^{\mathrm{TM}}$ software PCR design feature was used for designing primers. When primers were constructed with flanking regions, melting temperature of annealing part was kept above $55^{\circ} \mathrm{C}$, and with the flanking region it was kept above $65^{\circ} \mathrm{C}$. High secondary structure propensities were avoided. Primers were ordered from Sigma-Aldrich Chemie GmbH (Steinheim, Germany) as desalted oligonucleotides.

\subsubsection{Polymerase Chain Reaction (PCR)}

PCR was performed for amplification of DNA fragments from templates with desired changes and appropriate restrictions enzyme sites for introducing them into a vector backbone (Mullis et al., 1986) (Hutchison et al., 1978). PCR Enzyme PfuS triple mix components were recombinantly expressed in E.coli and purified by Steffen Frey from our lab, diluted to the final mix concentrations in PfuS buffer $(200 \mathrm{mM}$ Tris/ $\mathrm{HCl}$ pH9, $250 \mathrm{mM} \mathrm{KCl}, 15 \mathrm{mM} \mathrm{MgSO}, 100 \mathrm{mM}$ $\left(\mathrm{NH}_{4}\right)_{2} \mathrm{SO}_{4}, 1 \%$ Tween-20, $\left.1 \mathrm{mg} / \mathrm{ml} \mathrm{BSA}\right)$. 


\begin{tabular}{|lll|}
\hline Protein & Expression vector & Concentration \\
\hline PfuS & pSF302 & $100 \mathrm{ng} / \mu \mathrm{l}$ \\
\hline Pyrococcus abyssi pyrophosphatase & pSF336 & $15 \mathrm{ng} / \mu \mathrm{l}$ \\
\hline Pyrococcus abyssi dUTPase & pSF337 & $2.5 \mathrm{ng} / \mu \mathrm{l}$ \\
\hline
\end{tabular}

Table 10-4 PfuS Triple Mix Components

PfuS stands for an improved version of thermostable proofreading Pfu polymerase from Pyrococcus furiosus. By fusion of Sac7D DNA binding module from Sulfolobus acidocaldarius, the enzyme gained a 10-fold increase in processivity (Yang and Wang, 2004).

A typical $100 \mu \mathrm{l}$ PCR reaction was performed with $100 \mathrm{ng}$ of template DNA, $1 \mu \mathrm{l}$ of PfuS triple mix, $2 \mu \mathrm{l}$ DMSO, $8 \mu \mathrm{l}$ of dNTP-mixture (2.5 mM of each dNTP), $20 \mu \mathrm{l}$ of 5X Phusion HF Buffer (New England Biolabs, Ipswich, MA, USA), $1 \mu \mathrm{l}$ reverse and forward primer (100 $\mu \mathrm{M}$ each), and volume was completed with $d_{d H_{2}} \mathrm{O}$. A SensoQuest lab cycler (Göttingen) was used for PCR reactions. A typical example for a PCR reaction protocol is the following:

\begin{tabular}{|c|c|c|c|c|}
\hline Step No & Step Name & Temperature $\left({ }^{\circ} \mathrm{C}\right)$ & Length & Repeat \\
\hline 1 & Initial Denaturation & 98.5 & $5^{\prime}$ & 1 \\
\hline 2 & Denaturation & 98.5 & $30 "$ & \multirow{3}{*}{3} \\
\hline 3 & Primer annealing & $55-60$ & $30 "$ & \\
\hline 4 & Extension & 68 & Variable & \\
\hline 5 & Denaturation & 98.5 & $30 "$ & \multirow{3}{*}{30} \\
\hline 6 & Primer annealing & $65-70$ & $30 "$ & \\
\hline 7 & Extension & 68 & Variable & \\
\hline 8 & Final extension & 68 & $10^{\prime}$ & 1 \\
\hline
\end{tabular}

Table 10-5 PCR Reaction Steps

The first three cycles have low annealing temperature to incorporate the flanking regions of primers into the template, and when it is completed, annealing temperature is raised and cycle is continued for 30 rounds. Extension time depends on the length of the PCR product; PCR product length divided by polymerase speed $2 \mathrm{~kb} / \mathrm{min}$ was used as a standard way to determine this time.

\subsubsection{Mutagenesis PCR}

\subsubsection{Bsal Mediated Mutagenesis}

Bsal is a restriction enzyme that cleaves outside of its recognition sequence and creates recognition sequence independent overhangs. This has been employed in challenging cloning 
projects (Engler et al., 2008). As depicted in Figure 10-1, two PCR products were created with two primers each, P1 and P2, P3 and P4. P1 carried a regular restriction site for the integration of the $3^{\prime}$ of the construct and P4 carried another one for the integration of the $5^{\prime}$ to the target vector. On P2 and P3, Bsal was introduced before and after the site of mutagenesis, with opposite orientation. When both products were cleaved with Bsal, compatible overhangs with mutation were created. It was still highly efficient to ligate two digested PCR products and digested vector backbone in a single ligation reaction.

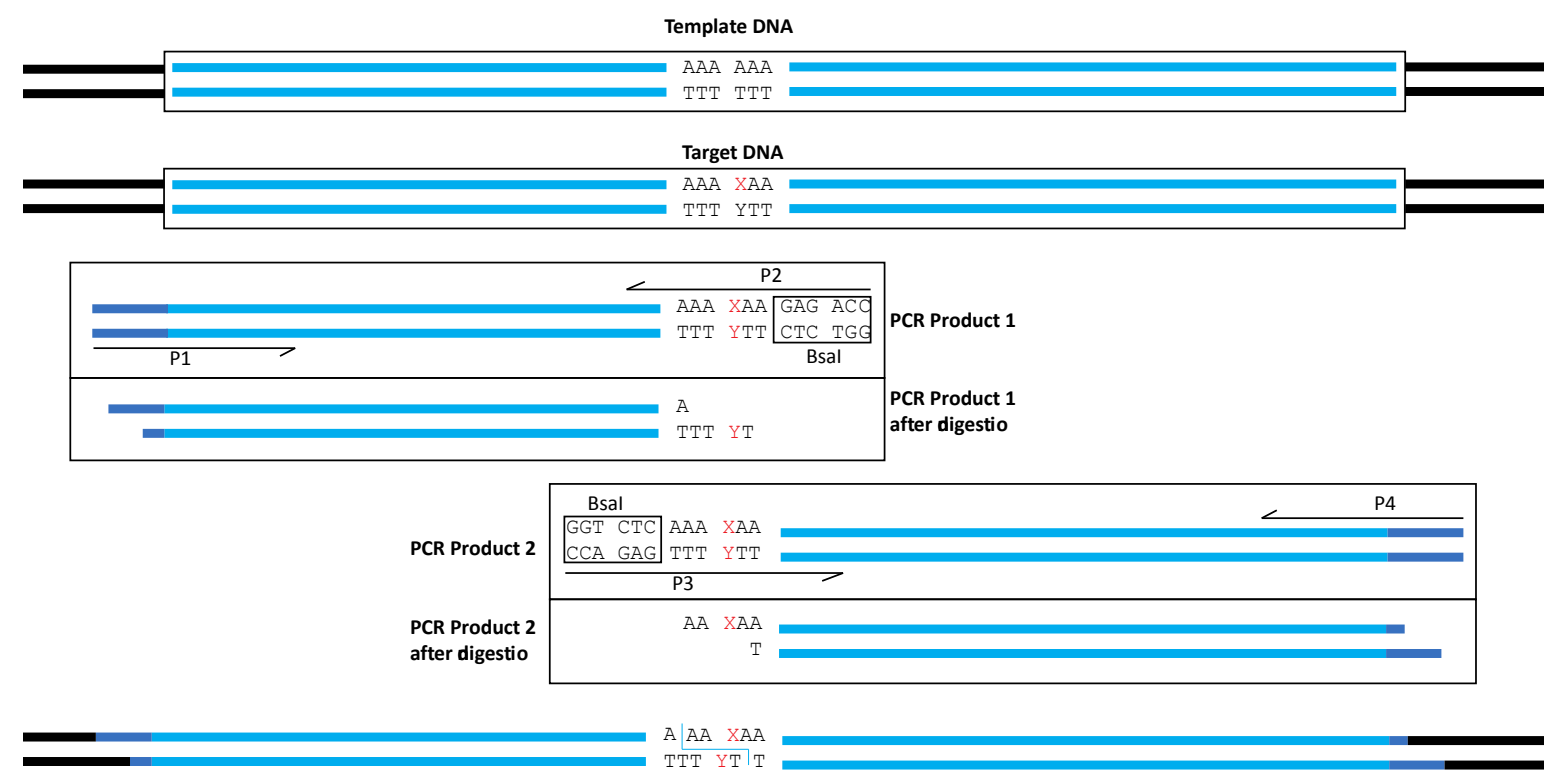

Figure 10-1 Bsal Mediated Mutagenesis

Since most of the existing constructs were already cloned via primers (P1 and P4), only two additional primers (P2 and P3) for each mutagenesis were needed.

When a Bsal site exists in the sequence of product of interest, it can be replaced by Bbsl, and when Bbsl also exists in the sequence, it is not possible to use this technique. For such cases following method is applied.

\subsubsection{Blunt End Ligation Mutagenesis}

Desired change in the sequence was coded on one of the primers, and two primers were adjacent to each other in the reverse directions, pointing their $3^{\prime} \mathrm{OH}$ ends away from each other. With these two primers, the whole plasmid can be amplified with one end bearing the mutation. Since the PfuS polymerase leaves blunt ends, ligation of this linear PCR product yields the desired DNA construct. After PCR, $1 \mu \mathrm{l}$ Dpnl was added to the PCR reaction to digest the methylated template DNA. The newly synthesized PCR product was not methylated, thus not digested. After Dpnl 
digestion, PCR product was purified over agarose gel and ligated. Since blunt end ligation is not as efficient as stick end ligation, this method was always the second choice.

\subsubsection{DNA Cleavage with Restriction Enzymes}

Restriction enzymes were bought from New England Biolabs (NEB, Ipswich, MA, USA) and used as recommended by NEB. When possible, either high fidelity enzymes or a selection of restriction enzymes was preferred (Table 10-6).

\begin{tabular}{|clcl|}
\hline Enzyme & $\begin{array}{l}\text { Recognition Sequence } \\
\text { and Cleavage Position }\end{array}$ & Enzyme & $\begin{array}{l}\text { Recognition Sequence } \\
\text { and Cleavage Position }\end{array}$ \\
\hline Acc65I & G/GTACC & Nhel & G/CTAGC \\
\hline Agel & A/CCGGT & Spel & A/CTAGT \\
\hline BamHI & G/GATCC & EcoRI & G/AATTC \\
\hline BspEI & T/CCGGA & Eagl & C/GGCCG \\
\hline HindIII & A/AGCTT & Bsal & GGTCTCN/NNNNN \\
\hline
\end{tabular}

Table 10-6 Preferred Restriction Enzymes

\subsubsection{DNA Gel Electrophoresis}

$\begin{array}{lll}50 x \text { TAE } & \text { DNA-ladder } & \text { Orange G Sample Buffer } \\ 242 \mathrm{~g} \text { Tris Base } & 50 \mathrm{ng} / \mu \mathrm{\mu l} \text { 1kb-Ladder (Thermo) } & 10 \mathrm{mM} \text { Tris/HCl pH } 8.0 \\ 57.1 \mathrm{ml} \text { Acetic acid } & \text { in Orange Sample Buffer (Gibco) } & 10 \mathrm{mM} \text { EDTA pH } 8.0 \\ 100 \mathrm{ml} \mathrm{0,5M} \text { EDTA pH } 8,0 & & 50 \%(\mathrm{w} / \mathrm{v}) \text { Glycerol } \\ \mathrm{ddH}_{2} \mathrm{O} \text { to } 1 \text { Litre } & & 25 \%(\mathrm{w} / \mathrm{v}) \text { Orange G }\end{array}$

DNA fragments were separated as described in (Sambrook and Russell, 2001) on agarose gels made of $1 \%$ agarose in TAE buffer. To visualize the DNA fragments $0.05 \mu \mathrm{g} / \mathrm{ml}$ ethidium bromide was added to the liquid agarose. DNA samples were combined with $1 / 10$ volume Orange $G$ sample buffer. After the run DNA bands were visualized on a UV Table (Benda Laborgeraete, Wiesloch), and excised.

\subsubsection{DNA Extraction From Agarose Gels}

For the purification of DNA fragments from excised agarose bands or from a solution Zymoclean Gel DNA recovery kit (Zymo Research, Freiburg) was used according to the manufacturer's instructions. 


\subsubsection{Determination of DNA Concentration}

The concentration of DNA solutions was determined via the extinction at $260 \mathrm{~nm}\left(E_{260}\right)$, with $E_{260}=$ 1.0 corresponding to $50 \mu \mathrm{g} / \mathrm{ml}$ double-stranded DNA (Sambrook and Russell, 2001). Measurements were done using ND-2000C spectrophotometer.

\subsubsection{Ligation of DNA Fragments into Vectors}

Vectors were treated with Fast Alkaline Phosphatase (FastAP, Fermentas) for $30 \mathrm{~min} 37^{\circ} \mathrm{C}$ in order to remove 5' phosphate groups and preventing the re-ligation of the vector. Digested and purified insert and vector fragments were ligated by T4 DNA ligase (100ng/ $\mu$; expressed in our lab by Steffen Frey from vector TB018) in a $10 \mu \mathrm{l}$ volume at RT for $1 \mathrm{~h}$ in $1 \mathrm{x}$ ligase buffer (10x ligase buffer: $500 \mathrm{mM}$ Tris pH7.5, $100 \mathrm{mM} \mathrm{MgCl}, 100 \mathrm{mM}$ DTT, $10 \mathrm{mM}$ ATP, $250 \mu \mathrm{g} / \mathrm{ml}$ BSA). $50 \mathrm{ng}$ of vector DNA was incubated without insert (re-ligation control), and with three fold molar excess of insert DNA. $1 \mu$ l of the ligation reaction was transformed into electrocompetent E.coli cells.

\subsubsection{Electroporation of E. coli Cells}

Electrocompetent cells were prepared by Gabriele Kopp according to the protocol form (Sambrook and Russell, 2001). To reach optimal transformation efficiency, aliquots of frozen electrocompetent $E$. coli cells were thawed slowly and kept on ice until electroporation. $45 \mu \mathrm{l}$ electro-competent $E$. coli cells and $1 \mu \mathrm{l}$ of ligation reaction were combined in an electroporation cuvette (165-2086; Bio-Rad, Hercules, CA, USA). Electroporation was performed using the GenePulser (Bio-Rad, Hercules, CA, USA) according to manufacturer's recommendations. Cells were recovered with $2 \mathrm{YT}$ medium ( $16 \mathrm{~g}$ Tryptone, $10 \mathrm{~g}$ Yeast extract, $5 \mathrm{~g} \mathrm{NaCl}$, in $1 / \mathrm{ddH}_{2} \mathrm{O}$ ) without antibiotics for $1 \mathrm{~h}$ at $37{ }^{\circ} \mathrm{C}$ and $200 \mu$ l of cells were plated on LB agar (10 g Tryptone, $5 \mathrm{~g}$ Yeast extract, $10 \mathrm{~g} \mathrm{NaCl}, 15 \mathrm{~g}$ Agar in $1 \mathrm{lddH_{2 }}$ O) containing the appropriate antibiotics for selection and incubated o/n at $37^{\circ} \mathrm{C}$.

Antibiotics were used with following concentrations for the selection of transformants; $100 \mu \mathrm{g} / \mathrm{ml}$ Ampicillin, $34 \mu \mathrm{g} / \mathrm{ml}$ Chloramphenicol, $25 \mu \mathrm{g} / \mathrm{ml}$ Kanamycin, and $50 \mu \mathrm{g} / \mathrm{ml}$ Spectinomycin.

\subsubsection{E. coli Strains}

BLR(DE3) (69053-3, Novagen), NEB Express 1 ${ }^{9}$ (C3037, New England Biolabs) cells were used for protein expression. For CRM1 expressions, BLR cells performed better in terms of protein yield and purity. For other proteins, NEB Express $1^{q}$ cells were used. NEB10-beta (C3019; New England Biolabs) cells were used for cloning. 


\subsubsection{DNA Purification From E. coli Cultures}

Cell cultures were started from single colonies in LB medium (10 g Tryptone, $5 \mathrm{~g}$ Yeast extract, 10 g NaCl in $1 \mathrm{lddH_{2 }}$ ) with appropriate antibiotics. Small-scale plasmid DNA preparations ("minipreps") were started with $8 \mathrm{ml}$ medium; large-scale plasmid DNA preparations ("midi-preps") were started with $250 \mathrm{ml}$ medium. Mini and Midi preps are processed using the two kits NucleoSpin Plasmid and NucleoBond PC100 (both Macherey-Nagel, Düren, Germany).

10.2.12 Bacterial Expression Constructs

\begin{tabular}{|ll|}
\hline Construct ID & Construct Content \\
\hline pKoKNES005 & H10ZZT-spRna1p373_386 \\
\hline pKoKNES006 & H10ZZT-spRna1p2-386 \\
\hline pKoKNES007 & H10ZZT-spRna1p1-383 \\
\hline pKoKNES069 & H14ZZbrSumo-spRna1p2-386 \\
\hline pKoKNES070 & H14ZZSumo-spRna1p2-383 \\
\hline pKoKNES072 & H14ZZSumo-spRna1pFull A384L \\
\hline pKoKNES073 & H14ZZSumo-spRna1pFull I386S \\
\hline pKoKNES090 & H14ZZSumo-scCrm1 \\
\hline pKoKNES100 & H14ZZSumo-mmCrm1 \\
\hline pKoKNES103 & H14Sumo-Avi-mmCRM1 \\
\hline pKoKNES114 & H10ZZT-elF2bNES \\
\hline pKoKNES115 & H10ZZT-elF2bNES O1L \\
\hline pKoKNES116 & H10ZZT-elF2bNES Xenla \\
\hline pKoKNES126 & H14AviSumo-hselF2beta \\
\hline pKoKNES127 & H14AviSumo-hselF2beta I103S \\
\hline pKoKNES132 & H14Sumo-Avi-scCRM1 \\
\hline
\end{tabular}

Table 10-7 Bacterial Expression Constructs

$\mathrm{H} 10$ and $\mathrm{H} 14$ stand for 10 or 14 histidine residues used as N terminal tag. T stands for TEV site. Avi stands for the Avi tag that is recognized by BirA and covalently modified with biotin.

\subsection{PROTEIN EXPRESSION AND PURIFICATION}

\subsubsection{Native Protein Expression and Purification}

All proteins were expressed in appropriate $E$. coli strains (10.2.10). Optimal expression conditions were determined for each protein individually. The following protocol was used as the common method of expression and purification, and when it was not efficient enough, expression and purification conditions were further optimized by using different E.coli strains, N-terminal tags and resuspension buffers. 


\subsubsection{Common Purification Conditions}

Frozen cells were thawed in hand warm water. Although freeze-thaw breaks cell walls, it is not enough since the DNA needs to be sheered by sonication for complete solubilization of the expressed proteins. Sonication was performed with Branson Sonifier settings $40 \%$ duty cycle and 10 output power in ice bucket for 2 minutes to compensate for the heat produced by the sonicator for $25 \mathrm{ml}$ of resuspended cells. If the volume was larger, sonication was performed with cycles of $2 \mathrm{~min}$ sonication and $1 \mathrm{~min}$ incubation in ice. The lysate was ultracentrifuged for $2 \mathrm{~h}$ in a T1250 rotor (Table 10-2) at 38,000 rpm to remove cell debris and large aggregates.

We expressed the proteins with an N-terminal histidine tag that consist of 10-14 histidine residues that can be used for affinity purification with $\mathrm{Ni}^{2+}$ immobilized beads. By this approach, histidine-tagged proteins can efficiently be purified from complex protein mixtures. The matrix (prepared by Dirk Görlich) was equilibrated with RS1 buffer. The amount of matrix added always depended on the level of protein expression. $\mathrm{Ni}^{2+}$-matrix was incubated with the lysate for $2 \mathrm{~h}$ at $4^{\circ} \mathrm{C}$ under rotation in the presence of $15 \mathrm{mM}$ imidazole in order to decrease the background binding to $\mathrm{Ni}^{2+}$-matrix from the bacterial lysate. .

The matrix was then let to settle, and after removing the supernatant, matrix was resuspended with RS1 buffer and applied to gravity flow column (volume approx. 10x matrix bed volumes). The resin was washed thoroughly with RS1 buffer containing $25 \mathrm{mM}$ imidazole to remove low affinity binders.

\subsubsection{Elution of Proteins from $\mathrm{Ni}^{2+}$ Matrix}

Elution of proteins from the $\mathrm{Ni}^{2+}$-matrix depended on the $\mathrm{N}$ terminal tag, and the purpose of the purification. If the protein was needed with the tag (e.g., enhancing solubility, immobilization on other matrices) it was eluted with imidazole that competes with histidine residues for $\mathrm{Ni}^{2+}$ binding. After the washing step, RS1 buffer containing $0.5 \mathrm{M}$ imidazole was added to the matrix in steps of $1 / 3$ of matrix volume with 2 min incubation, and each step is collected as a different fraction. These fractions were measured for their $A_{280}$ values, and peak fractions were pooled.

In cases where histidine tag was not further needed, proteins were eluted from the resin by digestion of the respective protease cleavage site between the His tag and the protein (e.g. sumo protease digestion for the His - Sumo tagged proteins). After the washing step, buffer in the resin column was quickly exchanged with RS1 buffer containing $5 \mathrm{mM}$ imidazole and $25 \mathrm{nM}$ untagged sumo protease (Expressed and purified by Steffen Frey from plasmid TB005). Resin was incubated for $1 \mathrm{~h} 4^{\circ} \mathrm{C}$ standing. Elution was done by slowly adding RS1 buffer (1 matrix volume) from the top and collecting the eluate in a single fraction. Elution contained the untagged protein in high 
concentration and purity with an insignificant contamination (1:1000 molar ratio) of the used protease.

Sucrose was added to the eluate to a final concentration of $250 \mathrm{mM}$ and the proteins were snapfrozen in liquid nitrogen in aliquots to prevent repeated freeze-thawing, and stored at $-80^{\circ} \mathrm{C}$. Samples of the uninduced and induced cells, post-sonication and -ultracentrifugation, flowthrough of the $\mathrm{Ni}^{2+}$-matrix and the eluted proteins were analyzed on a SDS-PAGE and visualized by Coomassie staining.

\subsubsection{Expression of Proteins with Biotinytlation}

Binding assays on streptavidin-agarose beads require biotinylated proteins as bait. For biotinylation, an N-terminal Avi-tag was included in the expression construct. Avi tag is a 15 amino acid long stretch (GLNDIFEAQKIEWHE), and in the expression construct it is flanked by flexible amino acid stretches. This tag can be recognized by biotin holoenzyme synthetase BirA and a biotin moiety is covalently conjugated to the avi-tag (Beckett et al., 1999). Protein expression vector was co-transformed with BirA expression vector (TB022, prepared by Steffen Frey). At the time of induction with IPTG, also $20 \mathrm{\mu g} / \mathrm{ml}$ biotin $(10 \mathrm{mg} / \mathrm{ml} \mathrm{pH} 7.0$ stock) is added to the culture. With this method in vivo biotinylation was achieved with $>99 \%$ efficiency.

\subsubsection{Determination of Protein Concentrations}

Protein concentrations were determined by conversion of the $A_{280}$ value with the calculated coefficients. The A280 value is measured with ND-2000C spectrophotometer that was blanked with RS1 buffer containing $5 \mathrm{mM}$ imidazole and $25 \mathrm{nM}$ untagged sumo protease.

A script written in Python Programming Language with Biopython Package (Cock et al., 2009) was used to extract the protein sequence from Lasergene SeqBuilder ${ }^{\mathrm{TM}}$ files (vector maps), check for sumo existence, and calculate the molecular weight and $A_{280}$ absorption coefficient based on the Equation 10-1 where $\mathrm{n}$ is the number of indicated amino acid in the sequence.

$$
\begin{aligned}
& \qquad \varepsilon=(n W x \mathbf{5 5 0 0})+(n Y x \mathbf{1 4 9 0})+(n C x \text { 125 }) \\
& \text { Equation 10-1 Absorption coefficient based on amino acid composition (Pace et al., 1995) }
\end{aligned}
$$

Values were calculated both for the full-length and sumo protease cleaved versions. With these values in hand, it is possible to calculate both the mass and molar concentrations.

\subsubsection{SDS-PAGE}

The method of discontinuous sodiumdodecylsulfate polyacrylamide gel electrophoresis (SDSPAGE) was performed according to standard protocols (Sambrook and Russell, 2001) that provide an up to date version of the original description (Laemmli, 1970). The composition of the gradient 
SDS-polyacrylamide gels prepared by Gabriele Kopp and Jürgen Schünemann is described below (Table 10-8). Equipment for the protocol (e.g., Glass plate sets, combs, electrophoresis chambers) had been built by the workshop of the MPI-BPC. Gels were run at $50 \mathrm{~mA}$ constant current until the bromophenol blue dye present in the sample buffer reached the bottom of the gel. Subsequently, proteins were fixed and stained by heating the gel in 3\% acetic acid and 1:100 dilution of the Coomassie stock solution ( $2 \%(w / v)$ Coomassie Brilliant Blue G250 in $50 \%$ Ethanol). Gels were destained in water and documented using the EPSON scanner.

\begin{tabular}{|l|l|l|l|}
\hline \multicolumn{1}{|c|}{ 'Heavy' Gel 16\% } & \multicolumn{1}{l|}{ 'Light' Gel 7.5\% } & \multicolumn{1}{l|}{ Stacking Gel 4.5\% } \\
\hline $2 \mathrm{M}$ Tris pH 8,8 & $200 \mathrm{ml}$ & $200 \mathrm{ml}$ & $100 \mathrm{ml}$ \\
\hline $0,5 \mathrm{M}$ Tris pH 6,8 & $40 \mathrm{ml}$ & $40 \mathrm{ml}$ & ------ \\
\hline $\mathrm{H}_{2} \mathrm{O}$ & ------ & ------- & $15 \mathrm{ml}$ \\
\hline $2 \mathrm{M}$ Sucrose & $32 \mathrm{ml}$ & $107 \mathrm{ml}$ & $68 \mathrm{ml}$ \\
\hline Glycerol $(87 \%)$ & $10 \mathrm{ml}$ & ------- & ------- \\
\hline $10 \%$ SDS & $8 \mathrm{ml}$ & ------- & ------- \\
\hline Rotiphorese Gel 30 & $2 \mathrm{ml}$ & $2 \mathrm{ml}$ & $1 \mathrm{ml}$ \\
\hline TEMED & $108 \mathrm{ml}$ & $51 \mathrm{ml}$ & $15 \mathrm{ml}$ \\
\hline APS 10\% & $120 \mu \mathrm{l}$ & $120 \mu \mathrm{l}$ & $100 \mu \mathrm{l}$ \\
\hline
\end{tabular}

Table 10-8 Gradient Gel Solutions 


\subsection{BINDING ASSAYS WITH CRM1}

\subsubsection{Binding Assays with Purified Components}

Binding assays with purified proteins were performed to test RanGTP dependent CRM1 interaction of candidate cargos or peptides. Different methods were used to pull down the complex. Regardless of the how the complex was pulled down, it was formed as described below.

All proteins were in aliquots and stored at $-80^{\circ} \mathrm{C}$. Proteins used in the assays were thawed once and was not used again with another freeze thaw cycle for other binding experiments. A 10X stock of binding buffer was prepared and used in all binding assays. Binding buffer was $50 \mathrm{mM}$ Tris/ $\mathrm{HCl}, 2 \mathrm{mM} \mathrm{Mg}(\mathrm{OAc})_{2}$ and prepared by 1:10 dilution of the 10X stock with ultrapure water (arium ${ }^{\circledR}$ pro UV, Sartorius, Gottingen), freshly thawed DTT was added to a final concentration of 5 $\mathrm{mM}$, and the buffer was filtered through $0.2 \mu \mathrm{M}$ filters (Whatman $\mathrm{GmbH}$, Dassel). For binding reaction and other steps of the assay, Mobicols (MoBiTec, Göttingen) were used as the reaction chamber. Mobicols are $700 \mu$ tubes with a conical bottom that can be plugged with filters designed for mobicols (35 $\mu \mathrm{m}$ pore size, MoBiTec, M513515).

Binding reaction was done in $500 \mu \mathrm{l}$ total volume in Mobicols. CRM1 concentration was $2 \mu \mathrm{M}$. Since CRM1 stock was $50 \mu \mathrm{M}$ with $250 \mathrm{mM} \mathrm{NaCl}$, CRM1 contribution to the final salt was $10 \mathrm{mM}$ $\mathrm{NaCl}$. For samples with RanGTP, $3 \mu \mathrm{M} \operatorname{Ran}_{5-180}$ Q69L GTP was used from a stock of $50 \mu \mathrm{M} \operatorname{Ran}_{5-180}$ Q69L GTP with $400 \mathrm{mM} \mathrm{NaCl}$. For each RanGTP sample $30 \mu \mathrm{l}$ of stock RanGTP was used with a final contribution of $24 \mathrm{mM} \mathrm{NaCl}$. For samples without RanGTP the same volume of RanGTP buffer was added to have same salt contribution. Candidate cargoes and peptides were used at a final concentration of $2 \mu \mathrm{M}$. Since purification of different proteins required different buffers and had different final protein yields, their contribution to final buffer conditions were calculated for each binding experiment. Final salt concentration was adjusted with a high salt buffer composed of $50 \mathrm{mM} \mathrm{NaCl}, 400 \mathrm{mM} \mathrm{NaCl}, 2 \mathrm{mM} \mathrm{Mg}(\mathrm{OAc})_{2} 5 \mathrm{mM}$ DTT.

When components were brought together in mobicols with a bottom plug and a screw cap, they were incubated in cold room at $4^{\circ} \mathrm{C}$ on SB3 rotator (Bibby Scientific, France) with a speed of 10 rpm for 2 hours. After 2 hours the respective affinity matrix was added.

\subsubsection{Binding Assays with ZZ-affibody Beads}

When candidate cargoes are expressed with an N-terminal ZZ tag, this tag can be used to bind them to $Z Z$ affibody. $Z$ in $Z Z$ stands for the synthetic IgG Fc region binding domain from the $B$ domain of the Staphylococcus aureus protein A. Affibody molecules are small proteins, engineered for specific protein interactions. An affibody made for $\mathrm{Z}$ domain binding (Wahlberg et 
al., 2003) was expressed and immobilized to functionalized silica beads (prepared by Dirk Görlich) by Steffen Frey. $5 \mathrm{mg}$ of ZZ affibody was immobilized on $1 \mathrm{ml}$ of functionalized silica beads. For

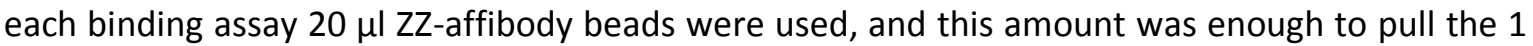
nmol of ZZ tagged candidate protein or peptide. When tested with ZZ-PKI NES peptide, all immobilized protein was competent in RanGTP dependent CRM1 binding.

Stocks of the ZZ-affibody beads were kept in $4.1 \mathrm{M}$ ammonium sulfate at $4^{\circ} \mathrm{C} .10 \%$ excess of the required amount of the beads were removed with pipette using a cut pipette tip, to not to harm the beads. Beads were placed in a mobicol, and washed 5 times with $500 \mu l$ binding buffer. After addition of buffer, mobicol was placed in a $2 \mathrm{ml}$ Eppendorf tube and centrifuged at $1000 \mathrm{rpm}$ at $4^{\circ} \mathrm{C}$ in a refrigerated table top centrifuge for $30 \mathrm{sec}$. At this low speed of centrifugation the sepharose-beads remained intact.

Beads were resuspended in 1:1 volume of binding buffer and $40 \mu \mathrm{l}$ of suspension was pipetted in the binding reaction. Binding reaction again was incubated in cold room at $4^{\circ} \mathrm{C}$ for 1 hour. After incubation, mobicols were unplugged and placed in $2 \mathrm{ml}$ Eppendorf tubes, and centrifuged at $1000 \mathrm{rpm}$ at $4^{\circ} \mathrm{C}$ in a refrigerated table top centrifuge. Flow-through was collected and beads were washed with $500 \mu \mathrm{l}$ binding buffer by $1000 \mathrm{rpm}$ centrifugation for $30 \mathrm{sec}$ at $4^{\circ} \mathrm{C} 2$ times. To get rid of the buffer that remained in the bead volume, a very short $(5-10 \mathrm{sec})$ centrifugation at $3000 \mathrm{rpm}$ was performed. Mobicols were placed in $1.5 \mathrm{ml}$ Eppendorf and $50 \mu \mathrm{l}$ SDS sample buffer was added. Mobicols were kept at room temperature for $5 \mathrm{~min}$ and centrifuged at $1000 \mathrm{rpm}$ for 1 $\mathrm{min}$ at room temperature tabletop centrifuge. Another $50 \mu \mathrm{l}$ SDS sample buffer was added and the mobicols were centrifuged at $1000 \mathrm{rpm}$ for $1 \mathrm{~min}$ at room temperature tabletop centrifuge. At the end $100 \mu$ l elution was collected in a single $1.5 \mathrm{ml}$ Eppendorf, and $10 \mu \mathrm{l}$ of each elution was analyzed on SDS-PAGE.

\subsubsection{Binding Assays with Streptavidin-agarose Beads}

For some binding assays either the candidate cargo or the CRM1 protein was biotinylated. Biotinylation was performed in vivo as explained in section 10.3.1.3. Biotin has a very high affinity for streptavidin homo tetramers $\left(\mathrm{K}_{\mathrm{d}}\right.$ in the order of $\left.10^{-14}\right)$. For pull downs of biotinylated proteins streptavidin-agarose beads (Sigma Aldrich) were used. 10\% excess of the required amount of the beads was removed with pipette using a cut pipette tip, in order not to harm the beads. Beads were placed in a mobicol, and washed 5 times with $500 \mu$ l binding buffer. After addition of buffer, mobicol was placed in a $2 \mathrm{ml}$ Eppendorf tube and centrifuged at $1000 \mathrm{rpm}$ at $4^{\circ} \mathrm{C}$ in a refrigerated table top centrifuge for $30 \mathrm{sec}$. At this low speed of centrifugation the sepharose-beads remained intact. 
Streptavidin agarose beads were resuspended in 1:1 volume of binding buffer and $40 \mu$ of suspension was pipetted in the binding reaction. Binding reaction was incubated in cold room at $4^{\circ} \mathrm{C}$ for 1 hour. After incubation, mobicols were unplugged and placed in $2 \mathrm{ml}$ Eppendorf tubes, and centrifuged at $1000 \mathrm{rpm}$ at $4^{\circ} \mathrm{C}$ in a refrigerated table top centrifuge. Flow-through was collected and beads were washed with $500 \mu$ l binding buffer by $1000 \mathrm{rpm}$ centrifugation for 30 $\sec$ at $4^{\circ} \mathrm{C} 2$ times. To get rid of the buffer that remained in the bead volume, a very short (5-10 sec) centrifugation at $2000 \mathrm{rpm}$ was performed. Mobicols were placed in $1.5 \mathrm{ml}$ Eppendorf tubes and $50 \mu \mathrm{ISDS}$ sample buffer was added. Tubes were placed on thermo shaker at $37^{\circ} \mathrm{C}$, and after an initial $1050 \mathrm{rpm}$ shake for $5 \mathrm{sec}$, they were incubated for $5 \mathrm{~min}$ at $350 \mathrm{rpm}$ shaking. If the initial high speed shaking was not done, bead would fail to mix with the SDS sample buffer and settle at the bottom. Mobicols in $1.5 \mathrm{ml}$ Eppendorf tubes were centrifuged at $1000 \mathrm{rpm}$ for $1 \mathrm{~min}$ at room temperature tabletop centrifuge. SDS sample buffer was kept at $37^{\circ} \mathrm{C}$, and another $50 \mu \mathrm{SDS}$ sample buffer was added and centrifuged at $1000 \mathrm{rpm}$ for $1 \mathrm{~min}$ at room temperature tabletop centrifuge. At the end $100 \mu \mathrm{l}$ elution was collected, and $10 \mu \mathrm{l}$ of each elution was analyzed on SDS-PAGE.

Streptavidin-biotin interaction is very strong and needs denaturing conditions to fully dissociate biotin from streptavidin. With this elution method, all of the prey material was retrieved, but only a partial dissociation of biotin-cargo or biotin-CRM1 was possible. To check immobilized protein levels and control if any prey was left on the beads, $20 \mu$ streptavidin agarose beads were boiled in $100 \mu \mathrm{l}$ SDS sample buffer and $10 \mu \mathrm{l}$ was analyzed on SDS-PAGE.

\subsection{PULL DOWN FROM CYTOPLASMIC EXTRACT WITH CRM1}

\subsubsection{Preparation of Cytoplasmic Extracts}

Cytoplasmic HeLa extracts were kindly provided by Lührmann Lab, Department of Cellular Biochemistry, MPI-BPC, and prepared with the following protocol modified from (Wahlberg et al., 2003). Monolayer of HeLa cells at $80 \%$ confluence was harvested by trypsinization. Cells are pelleted at 2000rpm for $5 \mathrm{~min}$ and washed 3 times with phosphate-buffered Saline (PBS: $130 \mathrm{mM}$ $\mathrm{NaCl}, 20 \mathrm{mM} \mathrm{K}_{2} \mathrm{HPO}_{4} / \mathrm{KH}_{2} \mathrm{PO}_{4}, \mathrm{pH}$ 7.4) for 10 min each. Cells were pelleted again and cell pellet was weighed. Packed cell volume ( $\mathrm{ml}$ ) was calculated by multiplying cell weight in gr by 0.96 . Total cell number was calculated by multiplying packed cell volume by $0.03 \times 10^{10}$. Cells were resuspended in 1.25 times the volume of packed cell volume of $\mathrm{MC}$ buffer $(10 \mathrm{mM} \mathrm{HEPES} / \mathrm{KOH} \mathrm{pH}$ 7.6, $10 \mathrm{mM}$ KOAc, $0.5 \mathrm{mM}$ MgOAc, $5 \mathrm{mM}$ DTT, 1x complete EDTA free proteinase inhibitor). Suspension was incubated 5 min on ice at $4^{\circ} \mathrm{C}$ and dounced 18 times using cell homogenizer. 
Homogenate was pelleted in Corex tubes at $13000 \mathrm{rcf}$ in SS34 rotor for 5 min. Supernatant was taken and frozen in liquid nitrogen, stored at $-80^{\circ} \mathrm{C}$.

This extracts were subjected to ultracentrifugation at $42000 \mathrm{rpm}$ in $55 \mathrm{~A}$ rotor at $4^{\circ} \mathrm{C}$ for $1 \mathrm{~h}$. Supernatant was collected, aliquoted, frozen in liquid nitrogen, and stored at $-80^{\circ} \mathrm{C}$.

\subsubsection{Preparation of Cytoplasmic SILAC HeLa Extracts}

Cytoplasmic SILAC HeLa extract were kindly supplied by Miroslav Nikolov (Mass Spectrometry Research Group, MPI-BPC). HeLa S3 cells were grown in lysine- and arginine-deficient Dulbecco's modified Eagle's medium supplemented with 10\% dialyzed fetal bovine serum (PAA, Pasching, Austria). One cell population was supplemented with natural L-lysine and L-arginine (Sigma, Munich, Germany) and another with heavy isotope labeled 4,4,5,5- $\mathrm{d}_{4}$-L-lysine and ${ }^{13} \mathrm{C}_{6}$-L-arginine (Euriso-Top, Saint-Aubin Cedex, France) generating mass shifts of 4 and $6 \mathrm{Da}$, respectively. Cells were grown for at least six passages at smaller volumes and then expanded to $2 \mathrm{I}$ in spinner flasks $\left(0.5-1.0 \times 10^{6}\right.$ cells $/ \mathrm{ml}$ ) (Ong and Mann, 2006). The cells were then transferred to a 5 I fermenter (Applikon, Schiedam, Netherlands) and grown under standard conditions $\left(2.5-5.0 \times 10^{6}\right.$ cells $\left./ \mathrm{ml}\right)$. Harvested cells were used to prepare cell extracts. Cells are pelleted at 2000rpm for 5 min and washed 3 times with phosphate-buffered Saline (PBS: $130 \mathrm{mM} \mathrm{NaCl}, 20 \mathrm{mM} \mathrm{K} \mathrm{K}_{2} \mathrm{HPO}_{4} / \mathrm{KH}_{2} \mathrm{PO}_{4}, \mathrm{pH}$ 7.4) for $10 \mathrm{~min}$ each. Cells were pelleted again and cell pellet was weighted. Packed cell volume

$(\mathrm{ml})$ was calculated by multiplying cell weight in gr by 0.96 . Total Cell number was calculated by multiplying packed cell volume by $0.03 \times 10^{10}$. Cells were resuspended in 1.25 times the volume of packed cell volume of MC buffer (10 mM HEPES/KOH pH 7.6, $10 \mathrm{mM} \mathrm{KOAc,} 0.5 \mathrm{mM} \mathrm{MgOAc}, 5$ $\mathrm{mM}$ DTT, $1 \mathrm{x}$ complete EDTA free proteinase inhibitor). Suspension was incubated $5 \mathrm{~min}$ on ice at $4^{\circ} \mathrm{C}$ and dounced 18 times using cell homogenizer. Homogenate was pelleted in Corex tubes at $13000 \mathrm{rcf}$ in SS34 rotor for $5 \mathrm{~min}$. Supernatant was taken and frozen in liquid nitrogen, stored at $80^{\circ} \mathrm{C}$.

This extracts were subjected to ultracentrifugation at $42000 \mathrm{rpm}$ in $\mathrm{S} 55 \mathrm{~A}$ rotor at $4^{\circ} \mathrm{C}$ for $1 \mathrm{~h}$. Supernatant was collected, aliquoted, frozen in liquid nitrogen, and stored at $-80^{\circ} \mathrm{C}$.

\subsubsection{CRM1 Affinity Chromatography with Cytoplasmic HeLa Extracts}

CRM1 Affinity Chromatography with Cytoplasmic HeLa Extracts was done to enrich the RanGTP dependent CRM1 binders of cytoplasmic HeLa proteins. This method was first used with cytoplasmic HeLa extract from Lührmann Lab, Department of Cellular Biochemistry, MPI-BPC to test and optimize the assay conditions. For the mass spectrometry analysis, we used this method with the cytoplasmic SILAC HeLa extracts from Miroslav Nikolov (Mass Spectrometry Research Group, MPI-BPC). 
All proteins and extracts were prepared as previously described and stored in aliquots $-80^{\circ} \mathrm{C}$. Proteins and extracts used in the assays were thawed once and was not used again with another freeze thaw cycle for other assay. A 10X stock of binding buffer was prepared and used in all binding assays. Binding buffer was $50 \mathrm{mM}$ Tris/ $\mathrm{HCl}, 2 \mathrm{mM} \mathrm{Mg}(\mathrm{OAc})_{2}$ and prepared by 1:10 dilution of the 10X stock with ultrapure water (arium ${ }^{\circledR}$ pro UV, sartorius, Gottingen), freshly thawed DTT was added to a final concentration of $5 \mathrm{mM}$, and the buffer was filtered through $0.2 \mu \mathrm{M}$ filters (Whatman $\mathrm{GmbH}$, Dassel). For binding reaction and other steps of the assay, Mobicols (MoBiTec, Göttingen) were used as the reaction chamber.

CRM1 immobilization was done on streptavidin-agarose beads. For each reaction $20 \mu$ of streptavidin-agarose beads were used. $10 \%$ excess of the total required amount of beads was taken with pipette using a cut pipette tip. Beads were placed in a mobicol, and washed 5 times with $500 \mu \mathrm{l}$ binding buffer. After addition of buffer, mobicol was placed in a $2 \mathrm{ml}$ Eppendorf tube and centrifuged at $1000 \mathrm{rpm}$ at $4^{\circ} \mathrm{C}$ in a refrigerated table top centrifuge for $30 \mathrm{sec}$. At this low speed of centrifugation the sepharose-beads remained intact.

For each reaction $0.5 \mathrm{nmol}$ of biotin-CRM1 was immobilized on $20 \mu \mathrm{l}$ of streptavidin-agarose beads. Total calculated amount of biotinylated CRM1 was added to mobicol with the beads, volume was completed to $500 \mu \mathrm{l}$ with a buffer containing $50 \mathrm{mM}$ Tris/ $\mathrm{HCl}, 100 \mathrm{mM} \mathrm{NaCl}, 2 \mathrm{mM}$ $\mathrm{Mg}(\mathrm{OAc})_{2}, 5 \mathrm{mM}$ DTT. For complete immobilization of biotinylated CRM1 on streptavidin-agarose beads, mobicol was kept in cold room for 1 hour on SB3 rotator (Bibby Scientific, France) at 10rpm. After incubation, beads in mobicols were washed 5 times with $500 \mu$ of buffer containing $50 \mathrm{mM}$ Tris/ $\mathrm{HCl}, 25 \mathrm{mM} \mathrm{NaCl}, 50 \mu \mathrm{M}$ biotin, $2 \mathrm{mM} \mathrm{Mg}(\mathrm{OAc})_{2}, 5 \mathrm{mM}$ DTT. After the addition of buffer, mobicols were kept for $1 \mathrm{~min}$ on ice and, placed in a $2 \mathrm{ml}$ Eppendorf tube and centrifuged at $1000 \mathrm{rpm}$ at $4^{\circ} \mathrm{C}$ in a refrigerated table top centrifuge for $30 \mathrm{sec}$. Beads were resuspended 1:1 volume of binding buffer and $40 \mu \mathrm{l}$ of suspension was pipetted in a new mobicol for each reaction. Aliquots of light and heavy extracts were thawed on ice, and centrifuged for $15 \mathrm{~min}$ at $4^{\circ} \mathrm{C}$ in S45A rotor at $37000 \mathrm{rpm}$.

CRM1 affinity chromatography was done in $500 \mu$ lotal volume in mobicols, with $20 \mu$ l of CRM1 immobilized streptavidin agarose beads. For reactions with RanGTP, $2 \mu \mathrm{M} \operatorname{Ran}_{5-180}$ Q69L GTP was used from a stock of $50 \mu \mathrm{M} \operatorname{Ran}_{5-180}$ Q69L GTP with $400 \mathrm{mM} \mathrm{NaCl}$. For each RanGTP sample $20 \mu \mathrm{l}$ of stock RanGTP was used with a final contribution of $20 \mathrm{mM} \mathrm{NaCl}$. For samples without RanGTP the same volume of RanGTP buffer was added to have same salt contribution. $100 \mu$ of centrifuged extract was added, final volume was brought to $500 \mu \mathrm{l}$ with binding buffer and final salt concentration was adjusted to $25 \mathrm{mM} \mathrm{NaCl}$. 
When components were brought together in mobicols with a bottom plug and a screw cap, they were incubated in cold room at $4^{\circ} \mathrm{C}$ on SB3 rotator at $10 \mathrm{rpm}$ for 3 hours. After incubation, mobicols were unplugged and placed in $2 \mathrm{ml}$ Eppendorf tubes, and centrifuged at $1000 \mathrm{rpm}$ at $4^{\circ} \mathrm{C}$ in a refrigerated table top centrifuge. Flow-through was collected and beads were washed with $500 \mu \mathrm{l}$ binding buffer with $25 \mathrm{mM} \mathrm{NaCl}$ by $1000 \mathrm{rpm}$ centrifugation for $30 \mathrm{sec}$ at $4^{\circ} \mathrm{C} 2$ times. To get rid of the buffer that remained in the bead volume, a very short $(5-10 \mathrm{sec})$ centrifugation at $2000 \mathrm{rpm}$ was performed. Mobicols were placed in $1.5 \mathrm{ml}$ Eppendorf tubes and $50 \mu \mathrm{l}$ SDS sample buffer was added. Eppendorf tubes were placed on thermo shaker at $37^{\circ} \mathrm{C}$, and after an initial 1050rpm shake for $5 \mathrm{sec}$, they were incubated for $5 \mathrm{~min}$ at $350 \mathrm{rpm}$ shaking. Mobicols in $1.5 \mathrm{ml}$ Eppendorf tubes were centrifuged at $1000 \mathrm{rpm}$ for $1 \mathrm{~min}$ at room temperature tabletop centrifuge. SDS sample buffer was kept at $37^{\circ} \mathrm{C}$, and another $50 \mu \mathrm{I}$ SDS sample buffer was added and centrifuged at $1000 \mathrm{rpm}$ for $1 \mathrm{~min}$ at room temperature tabletop centrifuge. At the end 100 $\mu l$ elution was collected, and $10 \mu$ l of each elution was analyzed on SDS-PAGE.

\subsubsection{Mass Spectrometry Analysis of Elution Fractions}

For forward experiment, 20ul of elutions from CRM1 beads with light HeLa extract without RanGTP, and heavy HeLa extract with RanGTP were put together. For reverse experiment, $20 \mu \mathrm{l}$ of elutions from CRM1 beads with heavy HeLa extract without RanGTP, and light HeLa extract with RanGTP were put together. Both combined elutions were concentrated to $20 \mu \mathrm{l}$ on rotational vacuum concentrator. Also 1:1 mix of input light and heavy extracts dissolved in SDS-sample buffer. Concentrated samples of forward and reverse experiment, and 1:1 mix of extracts were separated on $4-12 \%$ gradient SDS-PAGE gels (NuPAGE, Invitrogen, Carlsbad, CA) and stained with Colloidal Coomassie Blue $(0.08$ \% (w/v) Coomassie Brilliant Blue G250, 1.6 \% ( v/v) orthophosphoric acid, $8 \%$ (w/v) Ammonium sulphate, $20 \%$ (v/v) Methanol) (Neuhoff et al., 1988). Each gel lane was cut into 12 equal gel slices and proteins therein were in-gel digested with trypsin (Promega, Madison,WI) as described in (Neuhoff et al., 1988).

The rest of the protocol was performed by Samir Karaca (Mass Spectrometry Research Group, MPI-BPC). Extracted peptides were loaded into an in-house packed C18 trap column $(1.5 \mathrm{~cm}, 360$ $\mu \mathrm{m}$ outer diameter, $150 \mu \mathrm{m}$ inner diameter, Reprosil-Pur $120 \AA$, $5 \mu \mathrm{m}, \mathrm{C} 18-\mathrm{AQ}$, Dr. Maisch GmbH, Ammerbuch-Entringen, Germany) at a flow rate of $10 \mu \mathrm{l} / \mathrm{min}$. Retained peptides were eluted and separated on an analytical C18 capillary column $(15 \mathrm{~cm}, 360 \mu \mathrm{m}$ outer diameter, $75 \mu \mathrm{m}$ inner diameter, Reprosil-Pur $120 \AA$, $5 \mu \mathrm{m}, \mathrm{C} 18-\mathrm{AQ}$, Dr. Maisch GmbH, Germany) at a flow rate of 300 $\mathrm{nl} / \mathrm{min}$ with a gradient from $5 \%$ to $38 \%$ acetonitrile in $0.1 \%$ formic acid for 50 min using an Agilent 1100 nano-flow LC system (Agilent Technologies, Santa Clara, CA) coupled to an LTQ-Orbitrap Velos hybrid mass spectrometer (Thermo Electron, Bremen, Germany). The LTQ-Orbitrap Velos 
was operated in data-dependent mode and survey scans were acquired in the Or- bitrap $(\mathrm{m} / \mathrm{z}$ $350-1600$ ) with a resolution of 30,000 at $m / z 400$ with a target value of $1 \times$ e106. Up to 15 of the most intense ions with charges $\geq+2$ from the survey scan were sequentially isolated for collisioninduced dissociation with normalized collision energy of 37. Dynamic exclusion was set to $60 \mathrm{~s}$ to avoid repeating the sequencing of peptides. Each sample was analyzed in two technical replicates.

\subsubsection{Data and Bioinformatics Analysis}

Raw MS files from the LTQ- Orbitrap Velos were analyzed using MaxQuant software (version 1.3.0.5 (35) with Andromeda search engine. Peak lists generated by MaxQuant software were searched against the Uniprot Human protein database (downloaded on 10 July 2013, containing 88,354 entries) supplemented with common contaminants (e.g. keratins, serum albumin) and concatenated with the reverse sequences of all entries. MaxQuant search parameters were as follows: carbamidomethylation of cysteine was set as a fixed modification, whereas oxidation of methionine and $\mathrm{N}$-terminal protein acetylation were set as variable modifications; tryptic specificity with no proline restriction and up to two missed cleavages was used. The MS survey scan mass tolerance was $6 \mathrm{ppm}$ and for MS/MS 0.5 Da. Only peptides with a minimal length of five amino acids were considered for identification. The false discovery rate was set to $1 \%$ at both the peptide and the protein level. "Re-quantify" was enabled, and "keep low scoring versions of identified peptides" was disabled. Quantification of SILAC pairs was performed with a minimum ratio count of two by considering unique and razor peptides. To generate results with a high confidence interval, two biological replicates were performed, and each biological replicate was analyzed twice. To avoid false positives due to the experimental workflow, label-swap experiments were performed. Proteins behaving adversely in forward and reverse labeling experiments were excluded from the analysis.

Tab-delimited text file output from MaxQuant (proteinGroups.txt) was imported in R statistical Environment without pre-processing. All "Reverse" and "Contaminant" entries were excluded from further analysis. Non-normalized enrichment ratios in both label-swap experiments are represented in $\log 2$ scale.

The enrichment analysis for GO MF, BP and CC (Ashburner et al., 2000) were done for significant list with respect to HeLa proteome (Nagaraj et al., 2011) by the "conditional hypergeometric test" available in the GOstats package (Falcon and Gentleman, 2007) in the R statistical environment (R Development Team, 2012). KEGG pathway (Kanehisa and Goto, 2000) enrichment analysis was done in the same way, except that the hypergeometric test was used and the reference set was complete human KEGG annotations. 


\subsection{TRANSIENT HELA CELL TRANSFECTIONS}

GFP fusions of spRNA1 and PKI versions were prepared with a modified pEGFP-C1 (Invitrogen, Carlsbad, CA) vector. Each construct was co-transfected with vector coding for mCherry fusion of elF2 $\beta 65-114$, that has an NLS and NES. This construct from Chandini Kadian was used as the positive control.

\begin{tabular}{|ll|}
\hline Construct ID & Construct Content \\
\hline pKoKeu004 & eGFP-superPKI NES 3 \\
\hline pKoKeu005 & eGFP-PKI NES \\
\hline pKoKeu006 & eGFP-PKINESp4A \\
\hline pKoKeu008 & eGFP-spRna1p \\
\hline pKoKeu011 & eGFP-spRna1p2-376 \\
\hline pKoKeu035 & eGFP-SV40NLS-spRna1p \\
\hline pKoKeu038 & eGFP-SV40NLS-spRna1p2-376 \\
\hline pCK118 & mCherry-elF2beta65-114 \\
\hline
\end{tabular}

Table 10-9 Eukaryotic transfection constructs

1x $10^{4}$ HeLa cells were plated on each coverslip in a 24 well plate $\left(1.88 \mathrm{~cm}^{2}\right.$ growth area/ coverslip). Next day, when cells reach a confluency of $50-80 \%$, transfection was performed. Transfection was done with $0.1 \mu \mathrm{g}$ of each construct with FuGENE6 (Promega, Madison,WI) reagent. Transfections were done by Heinz-Jürgen Dehne with the provided guidelines from the producer. After $24 \mathrm{~h}$, cells on coverslips were washed 2 times with $3 \mathrm{ml}$ of PBS, and cells on coverslips were fixed with 5 min incubation in $4 \%$ paraformaldehyde. Excess paraformaldehyde was removed with another PBS wash including DAPI. $2.5 \mu \mathrm{l}$ Vectashield (Vector Laboratories, CA, USA) was placed on glass slides, and coverslips were placed on the glass slide with cells facing the Vectashield drop. This way, the cells were in between the coverslips and the glass slides. Confocal microscopy images were recorded using a Leica TCS SP5 Laser scanning microscope equipped with a HC PL APO 20x glycerol objective (Leica GmbH, Mannheim). 


\section{ABBREVIATIONS}

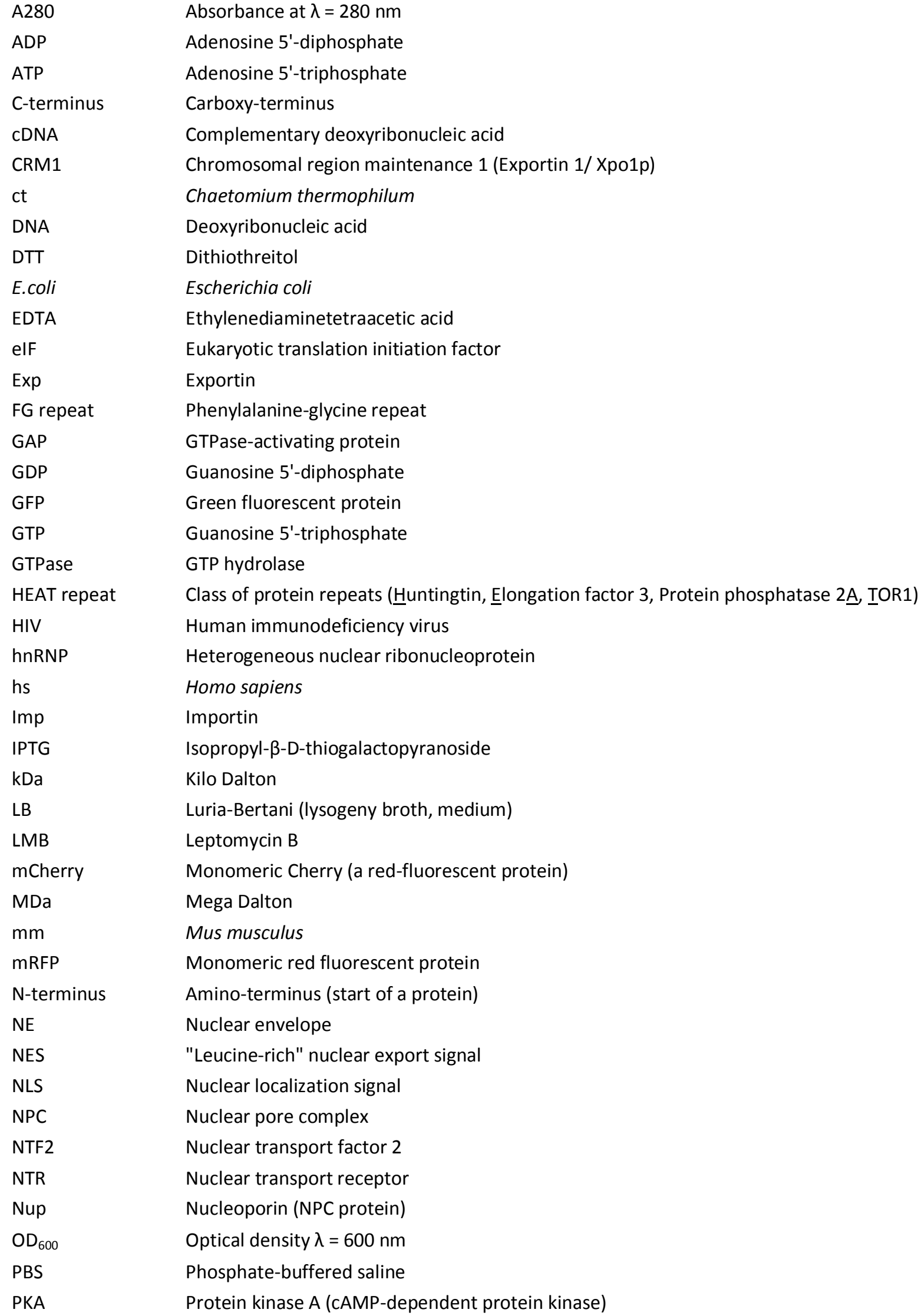

A280

ADP

ATP

C-terminus

CDNA

CRM1

ct

DNA

DTT

E.coli

EDTA

elF

Exp

FG repeat

GAP

GDP

GFP

GTP

GTPase

HEAT repeat

HIV

hnRNP

hs

Imp

IPTG

kDa

LB

LMB

mCherry

MDa

$\mathrm{mm}$

mRFP

$\mathrm{N}$-terminus

NE

NES

NLS

NPC

NTF2

NTR

Nup

$\mathrm{OD}_{600}$

PBS

PKA

Absorbance at $\lambda=280 \mathrm{~nm}$

Adenosine 5'-diphosphate

Adenosine 5'-triphosphate

Carboxy-terminus

Complementary deoxyribonucleic acid

Chromosomal region maintenance 1 (Exportin 1/ Xpo1p)

Chaetomium thermophilum

Deoxyribonucleic acid

Dithiothreitol

Escherichia coli

Ethylenediaminetetraacetic acid

Eukaryotic translation initiation factor

Exportin

Phenylalanine-glycine repeat

GTPase-activating protein

Guanosine 5'-diphosphate

Green fluorescent protein

Guanosine 5'-triphosphate

GTP hydrolase

Class of protein repeats (ㅂuntingtin, Elongation factor 3, Protein phosphatase $2 \underline{A}$, $\underline{\text { TOR}} 1$ )

Human immunodeficiency virus

Heterogeneous nuclear ribonucleoprotein

Homo sapiens

Importin

Isopropyl- $\beta$-D-thiogalactopyranoside

Kilo Dalton

Luria-Bertani (lysogeny broth, medium)

Leptomycin B

Monomeric Cherry (a red-fluorescent protein)

Mega Dalton

Mus musculus

Monomeric red fluorescent protein

Amino-terminus (start of a protein)

Nuclear envelope

"Leucine-rich" nuclear export signal

Nuclear localization signal

Nuclear pore complex

Nuclear transport factor 2

Nuclear transport receptor

Nucleoporin (NPC protein)

Optical density $\lambda=600 \mathrm{~nm}$

Phosphate-buffered saline

Protein kinase A (cAMP-dependent protein kinase) 
PKI

PMSF

Ran

RanBP

RanGAP

RanGEF

Ras

RCC1

rpm

SC

SDS-PAGE

$\mathrm{sp}$

SPN1

SV40

TEV

Tris

z

Protein kinase $\mathrm{A}$ inhibitor

Phenylmethylsulfonyl fluoride

Ras-related nuclear antigen

Ran-binding protein

RanGTPase-activating protein

Ran guanine nucleotide exchange factor

Rat sarcoma

Regulator of chromosome condensation 1 (see also "RanGEF")

Rounds per minute

Schizosaccharomyces pombe

Sodium dodecyl sulfate-polyacrylamide gel electrophoresis

Saccharomyces cerevisiae

Snurportin 1

Simian virus 40

Tobacco etch virus

2-amino-2-hydroxymethyl-1,3-propanediol

(in "zz") IgG-binding domain of the Staphylococcal protein A 


\section{APPENDIX}

\subsection{PYTHON SCRIPTS}

\subsubsection{PKI-type NES Prediction}

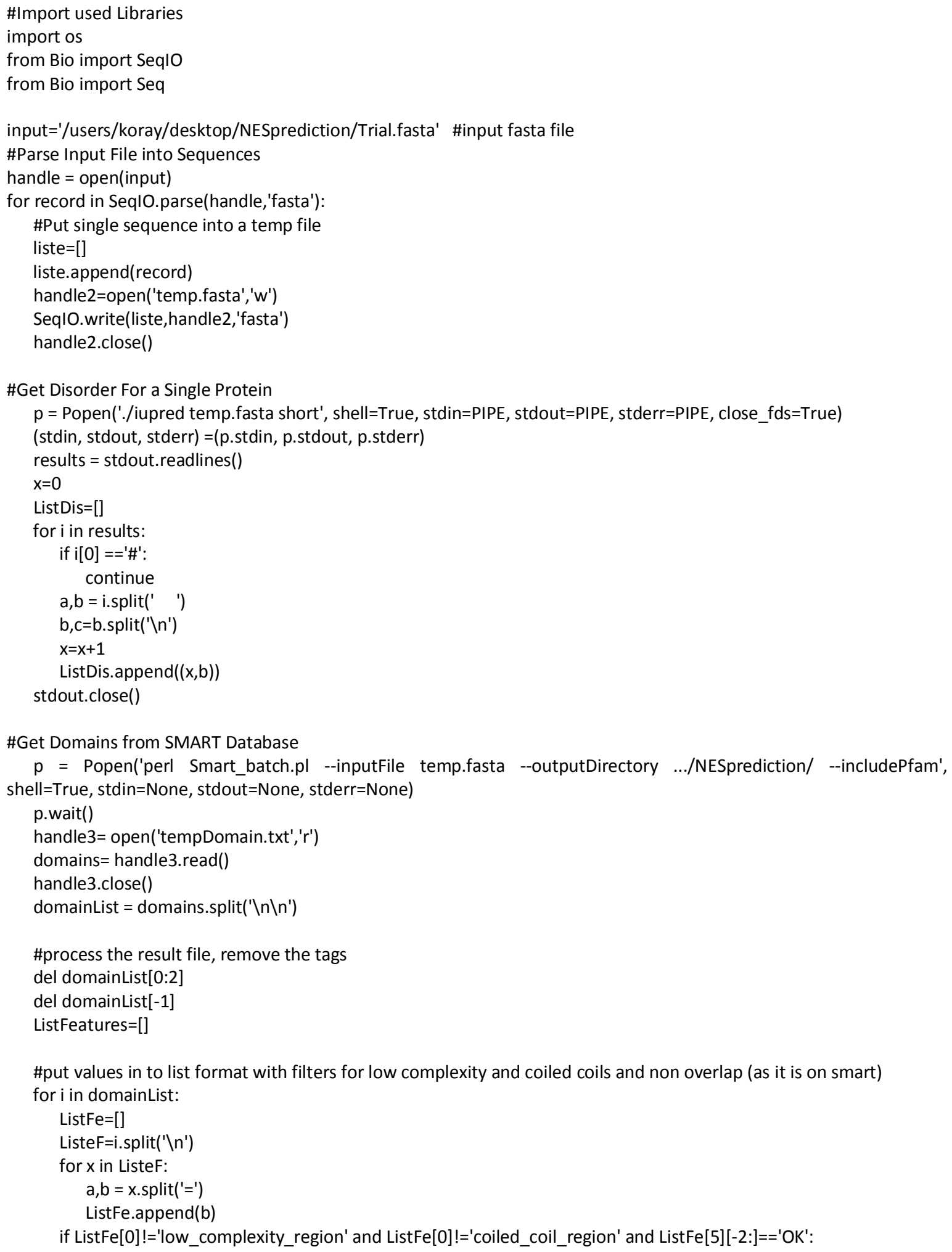


ListFeatures.append(ListFe)

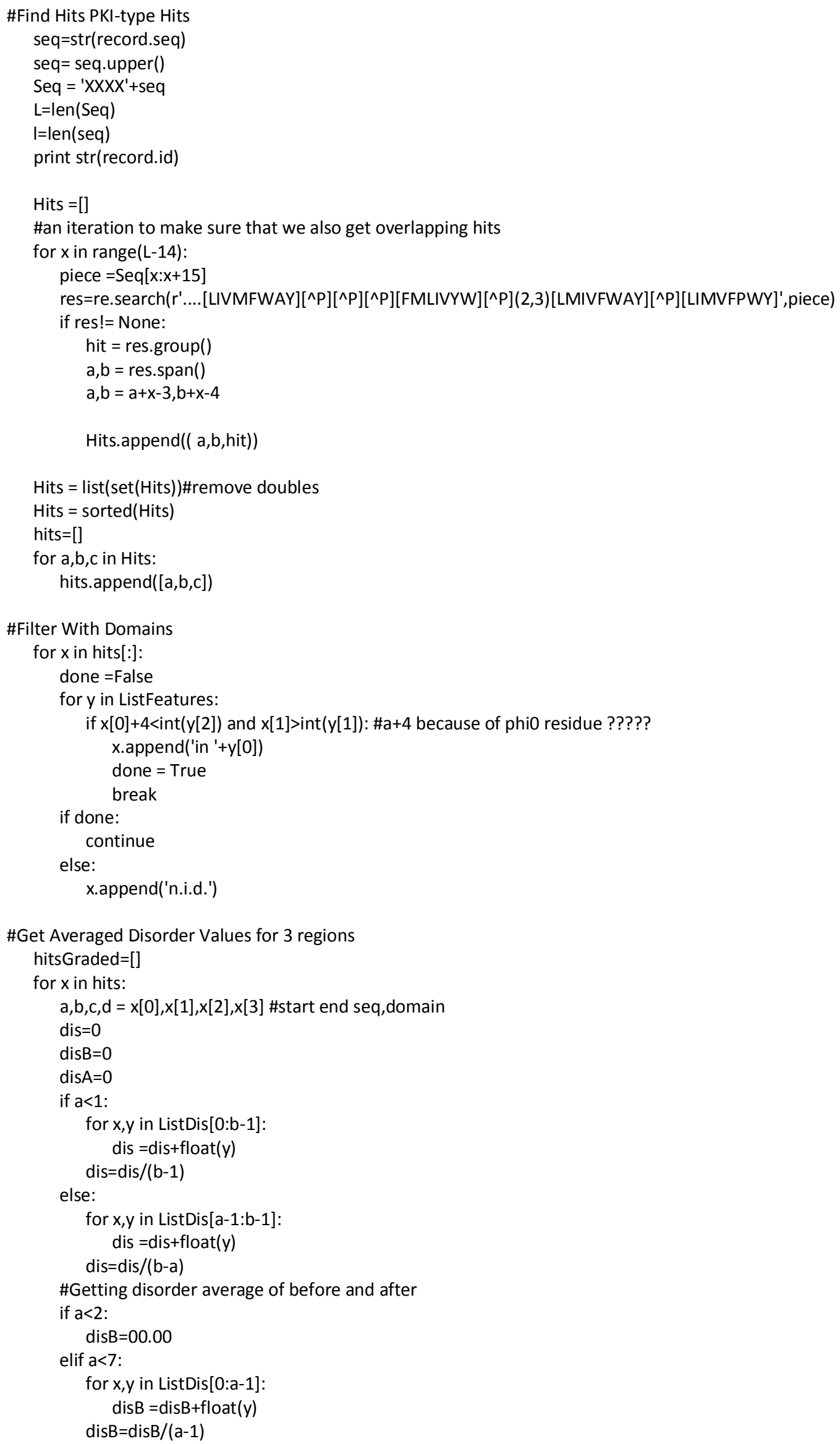


else:

for $x, y$ in ListDis[a-7:a-1]:

$\operatorname{dis} B=\operatorname{dis} B+f l o a t(y)$

$\operatorname{dis} B=\operatorname{dis} B /(6)$

if $\mathrm{l}==\mathrm{b}$ :

$\operatorname{dis} A=00.00$

elif I-b<6:

for $x, y$ in ListDis[b:]: $\operatorname{dis} A=\operatorname{dis} A+f l o a t(y)$

$\operatorname{dis} A=\operatorname{dis} A /(I-b)$

else:

for $x, y$ in ListDis[b-1:b+5]:

$\operatorname{dis} A=\operatorname{dis} A+f l o a t(y)$

$\operatorname{dis} A=\operatorname{dis} A /(6)$

hitsGraded.append([disB,dis,disA,c,a,b,d]) \#disorder of seq,disorder of 6 aa before, disorder of 6aa after,sequence,start,end,domain

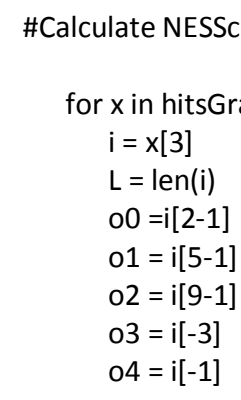

\#Scoring for $\mathrm{O} 0$ and its neighbors

if o0 in 'IVML':

$\mathrm{s} 0=0.5$

elif o0 is 'FAYW':

$\mathrm{s} 0=0.25$

else:

$\mathrm{s} 0=0$

if $i[0]$ in 'DE':

$\mathrm{s} 0=\mathrm{s} 0+0.5$

if i[2] in ' $D E$ ':

$\mathrm{s} 0=\mathrm{s} 0+0.5$

if $i[3]$ in ' $D E$ ':

$\mathrm{s} 0=\mathrm{s} 0+0.5$

if $s 0<1$ :

$\mathrm{s} 0=1$

else:

$\mathrm{s} 0=$ float $(\mathrm{s} 0)$

\#Scoring for 01 strength

if 01 is ' $L$ ':

$s 1=10$

elif 01 is ' 1 ':

$\mathrm{s} 1=8$

elif o1 in 'VM':

$s 1=6$

elif o1 is ' $F$ ':

$\mathrm{s} 1=4$

elif o1 is ' $A$ ':

$s 1=2$

elif o1 in 'WY':

$s 1=1$

\#Scoring for 02 strength

if 02 in 'FML': 
$s 2=10$

elif o2 in 'IV':

$s 2=8$

elif 02 is ' $Y$ ':

$s 2=3$

elif o2 is ' $W$ ':

$s 2=1$

\#Scoring for 03 strength

if 03 in 'LM':

$s 3=10$

elif 03 is ' $I$ ':

$s 3=9$

elif 03 in ' $V$ ':

s3 $=7$

elif 03 in ' $F$ ':

s3 $=3$

elif o3 in 'WA':

$s 3=2$

elif 03 in 'YT':

s3 $=1$

\#Scoring for 04 strength

if 04 in ' $L$ ':

s4 = 10

if 04 in ' $I$ ':

$s 4=9$

elif 04 is ' $M$ ':

$s 4=8$

elif o4 in 'V':

$s 4=7$

elif 04 in ' $F$ ':

$s 4=5$

elif 04 in 'PYW':

$s 4=1$

Score $=\operatorname{int}\left(s 0^{*} s{ }^{*} s 2^{*} s 3^{*} s 4\right)$

x.append(Score)

\#Simplify the Disorder values

if $x[0]<0.25$ :

Disln=1

elif $x[0]<0.5$ :

Disln=2

else:

Dis $n=3$

if $x[1]<0.25$ :

DisSeq $=1$

elif $x[1]<0.5$ :

DisSeq $=2$

else:

DisSeq $=3$

if $x[2]<0.25$ :

DisOut=1

elif $x[2]<0.5$ :

DisOut=2

else:

DisOut $=3$

$x[0]=$ Disln

$x[1]=$ DisSeq

$x[2]=$ DisOut

Pri $="$

for em in $\mathrm{x}$ :

Pri $=$ Pri $+\operatorname{str}(e m)++^{\prime} \backslash t^{\prime}$

print Pri, '\n\n' 


\subsubsection{REV-type NES Prediction}

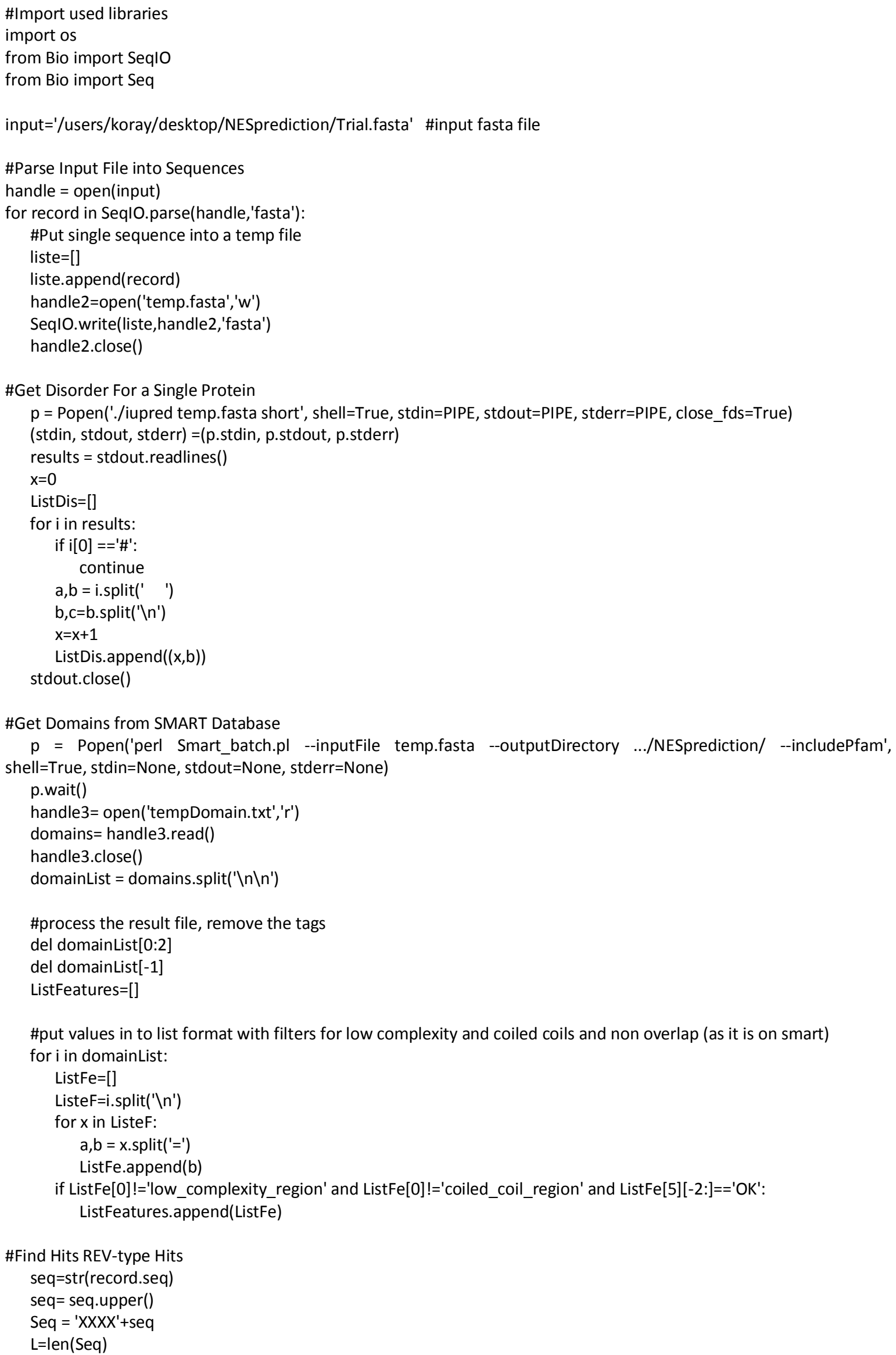




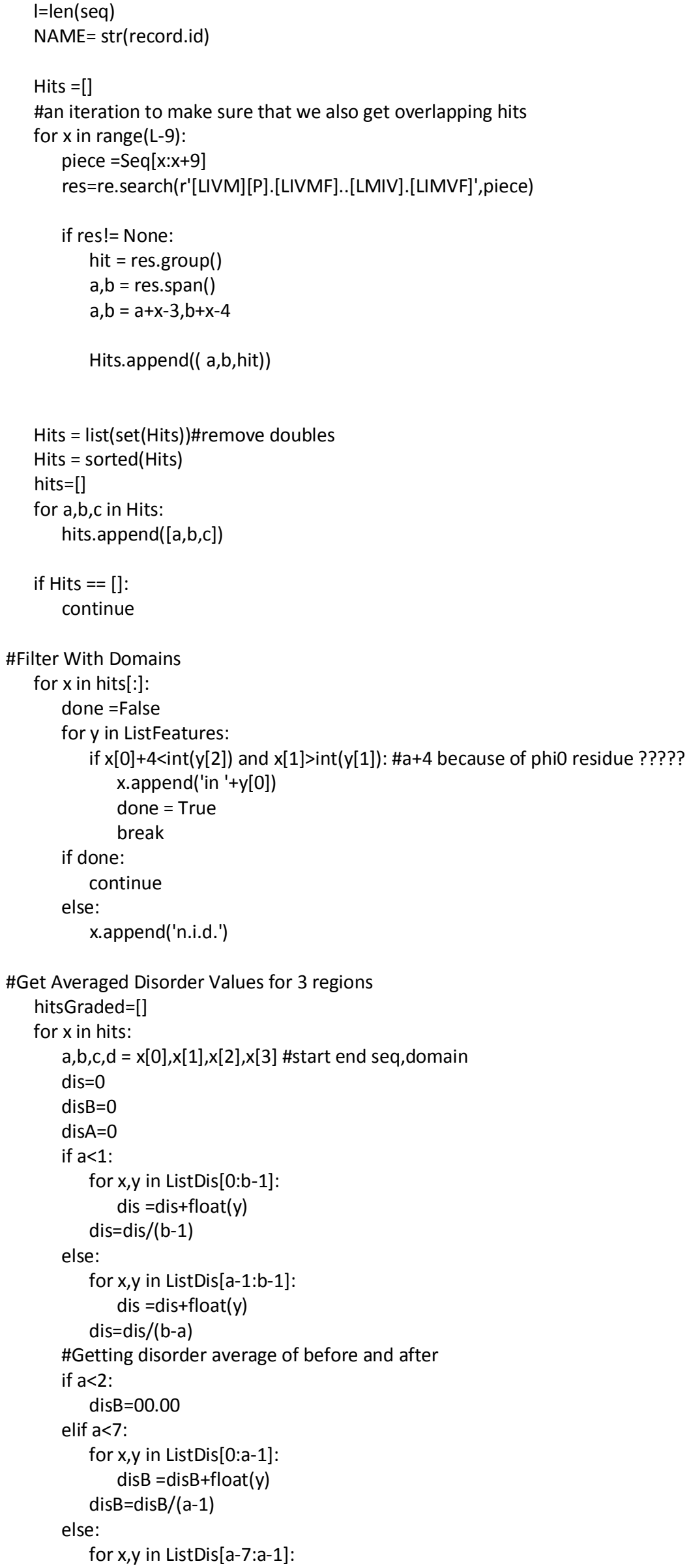




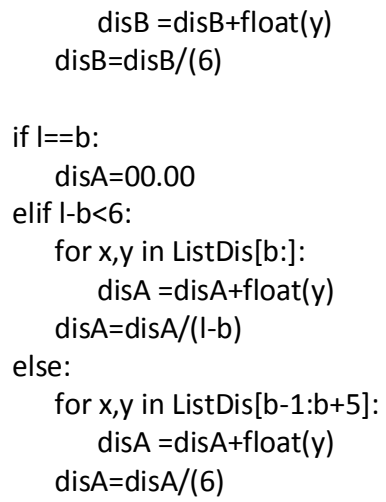

hitsGraded.append([disB,dis,disA,c,a,b,d]) \#disorder of seq,disorder of 6 aa before, disorder of $6 a a$ after,sequence,start,end,domain

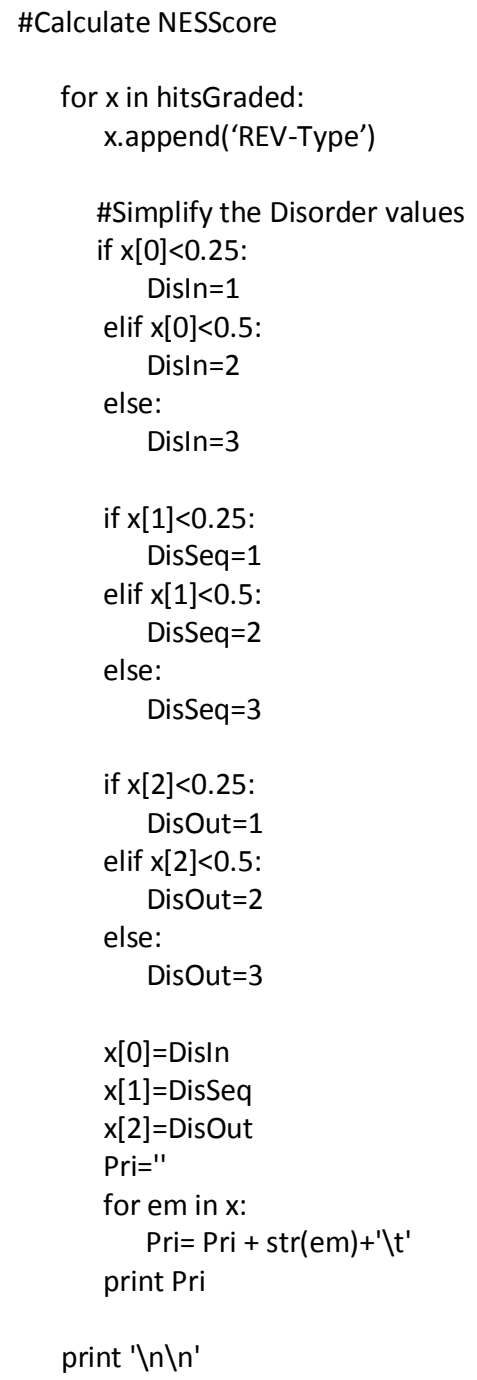




\subsection{PROTEIN IDENTIFIERS OF THE DATA SETS}

12.2.1 NESdb Proteins

\begin{tabular}{|l|l|l|l|l|l|}
\hline O95149 & O14746 & O15360 & Q99612 & Q8N720 & Q9BY84 \\
\hline Q96D46 & P24385 & Q8N668 & Q16828 & P15336 & P14373 \\
\hline P61925 & P35869 & P11388 & P03372 & P38936 & P25054 \\
\hline P04637 & P46108 & O15392 & P51587 & P67775 & O00221 \\
\hline P43487 & Q01094 & Q9UNH5 & P05230 & Q6UB99 & Q9UMX3 \\
\hline P38398 & P56693 & Q01658 & O75832 & Q96JZ2 & Q16236 \\
\hline P14635 & Q9UQL6 & P48436 & Q9NQS1 & Q9HC62 & Q9NYF0 \\
\hline Q00987 & P56524 & Q9HAP2 & O94916 & Q96T21 & P55265 \\
\hline Q13485 & Q16254 & Q9BYM8 & O43196 & Q86TB9 & Q7RTN6 \\
\hline O60716 & Q13043 & Q14457 & Q9H4D5 & Q96RS0 & P30740 \\
\hline Q06787 & Q9GZX7 & P46777 & Q9BRK4 & Q9H9S0 & Q15172 \\
\hline P42566 & Q05397 & Q9UGR2 & Q96K30 & Q9HBL8 & 095613 \\
\hline Q04206 & Q13568 & Q93052 & O15457 & O43707 & O00311 \\
\hline P42224 & O95644 & O96018 & P40692 & Q9Y3I1 & Q17RY0 \\
\hline Q14872 & P06748 & Q96C86 & Q14145 & P78545 & Q8IXJ6 \\
\hline Q9Y572 & Q02880 & O15519 & O15265 & Q9NRA8 & P28289 \\
\hline P25963 & Q99653 & P30291 & Q14140 & O96013 & Q9HCE7 \\
\hline Q14653 & P41970 & Q9BZB8 & Q13148 & Q9P286 & O00255 \\
\hline O15350 & Q14494 & Q9Y3M2 & Q00535 & Q00653 & P49023 \\
\hline Q15797 & Q13490 & Q9BVS4 & Q92688 & P42858 & Q81VI9-3 \\
\hline
\end{tabular}

Table 12-1 List of Uniprot IDs of human proteins of NESdb

\begin{tabular}{|l|l|l|l|l|l|}
\hline P14635 & P26651 & Q9UHL0 & Q15654 & P40424 & P17931 \\
\hline Q02750 & P41743 & P41134 & Q9UD71 & Q9NP71 & O00327 \\
\hline Q92664 & Q00994 & Q02363 & Q14012 & Q03052 & O00571 \\
\hline P49137 & P40763 & O60934 & P54646 & P06241 & Q92598 \\
\hline P00519 & P26196 & Q7Z2W4 & Q9UBK2 & Q9Y261 & Q92905 \\
\hline Q15942 & Q16236 & Q13887 & Q13574 & O15530 & O95863 \\
\hline 015534 & P41238 & Q6NXT1 & Q8IW41 & Q9H3M7 & O14867 \\
\hline O15055 & Q9NSV4 & Q9NUL3 & Q9NRF2 & Q6PIJ6 & O00401 \\
\hline P51178 & Q8NHY2 & Q99593 & O75553 & Q9UDY2 & \\
\hline
\end{tabular}


Table 12-2 List of Uniprot IDs of human ortholog proteins of NESdb

12.2.2 LMB Study Proteins

\begin{tabular}{|l|l|l|l|l|l|}
\hline Q13501 & B3KS26 & Q9NVU7 & Q71V88 & B4DY85 & B4DFI6 \\
\hline C9JDG0 & Q53EY3 & B4DH66 & B3KPH9 & B4DGH7 & P62917 \\
\hline Q9NVN8 & B5BTY4 & Q9Y2U5 & B4DHJ3 & O43818 & Q76N54 \\
\hline Q13895 & Q13867 & B4E303 & Q53GL4 & Q99873 & Q5VVD0 \\
\hline Q00653 & Q12769 & Q96CT7 & Q53RG0 & B7Z6Z3 & P46776 \\
\hline Q12800 & Q9BRP8 & E7EQZ4 & Q92973 & O00488 & B2R4K7 \\
\hline Q2NL82 & O00629 & A8K333 & A8K4W0 & Q9BQE6 & 095036 \\
\hline B3KT11 & Q9BZ95 & O15397 & Q92905 & B4DZW4 & Q9BYF5 \\
\hline B4DTT8 & Q6NVW7 & Q9UBB6 & A8K0B9 & Q2TAC2 & Q1JQ76 \\
\hline A8K066 & Q96EY4 & Q9BRJ6 & Q9H1A4 & B2RBA0 & Q6IBH6 \\
\hline D2CFK9 & Q9UHA3 & Q8N6M0 & Q9BV44 & Q2TA84 & Q53Z07 \\
\hline Q9H7E9 & Q8NCK5 & B4DTL6 & Q8TEX9 & B4DM84 & P61513 \\
\hline Q96QL0 & B2R823 & Q9H1C7 & Q8IYV2 & Q9UPW5 & P62888 \\
\hline P56537 & B3KM71 & B4DR01 & Q9UI30 & Q9NPD3 & Q53H34 \\
\hline Q5SRE5 & Q13823 & P13861 & Q53FF6 & Q9UJX2 & F8W727 \\
\hline Q04206 & Q9H8Y5 & Q56VW8 & Q6NW29 & Q8N1F7 & P18077 \\
\hline C9JEH3 & Q8TCG1 & Q5BKZ2 & B3KN10 & Q9UJ71 & B2R4C1 \\
\hline Q92529 & B5BUK7 & B3KTA3 & A8K4M5 & Q9Y2L1 & Q6IAX2 \\
\hline B3KMR5 & Q9BRP1 & Q9HBB9 & Q96KJ8 & B7Z5J8 & P49207 \\
\hline Q9NVB0 & B4DII5 & Q9H3S7 & Q76MU0 & P83881 & Q59EL2 \\
\hline A8K2F9 & Q53F09 & B7Z871 & Q5T1Z8 & Q0QEW2 & Q567U8 \\
\hline Q53FV3 & Q7L5N1 & Q99627 & Q06210 & Q59GS5 & Q6VY07 \\
\hline Q6Y7W6 & A8K3Q9 & A8K070 & & & \\
\hline
\end{tabular}

Table 12-3 List of Uniprot IDs of proteins from LMB Study

12.2.3 1265 Significant Hits of SILAC MS Data

\begin{tabular}{|l|l|l|l|l|l|}
\hline P35869 & Q9UMR2 & Q12774 & P62424 & P35269 & Q8WW12 \\
\hline Q14145 & P55039 & P16989 & P83731 & Q9Y606 & P53041 \\
\hline Q86TB9 & Q9C0B9 & Q99570 & P19525 & P09012 & P02545 \\
\hline Q93052 & P11274 & P10398 & Q9BRZ2 & Q96B26 & P49736 \\
\hline Q17RY0 & Q96GV9 & Q9Y3U8 & Q9BXP5 & P47755 & Q9NP77 \\
\hline
\end{tabular}


98

\begin{tabular}{|c|c|c|c|c|c|}
\hline Q2TAM5 & F5GWA6 & O15355 & Q8NHQ9 & Q6P6C2 & P26358 \\
\hline Q96D46 & Q15628 & P53618 & P62241 & Q9UNQ2 & Q14566 \\
\hline Q8IXJ6 & 060678 & Q9NWT1 & Q8TBB5 & Q8N163 & J3KTL2 \\
\hline Q9BVS4 & Q9UHB9 & P19784 & P13489 & Q9H9A5 & 043390 \\
\hline Q14653 & Q7Z739 & Q96HC4 & Q13627 & Q12986 & Q9Y2W1 \\
\hline P25963 & Q92572 & Q13347 & A6NIH7 & Q01844 & Q13123 \\
\hline Q13043 & Q6YHU6 & Q8TED0 & Q5T1V6 & Q8WX92 & E9PAV3 \\
\hline P30291 & Q9BQA1 & 015116 & P67809 & Q9Y224 & 014979 \\
\hline P25054 & Q8IWV7 & P46934 & P62081 & P09661 & P62913 \\
\hline Q99717 & Q96PU5 & P52434 & Q96N67 & 075794 & P23246 \\
\hline Q9NRA8 & 043933 & P39019 & J9JIC5 & Q00688 & Q9UKM9 \\
\hline Q9NQS1 & Q7LBC6 & B3КYA7 & Q9NZL4 & F8VVA7 & J3KN67 \\
\hline Q92688 & Q96QG7 & P55209 & Q2M389 & E9PC97 & Q27J81 \\
\hline P67775 & P25398 & P29597 & 043148 & B1AHD1 & Q15691 \\
\hline P42566 & P60228 & Q9BW66 & Q69YN2 & Q9H9A6 & Q9H307 \\
\hline 096013 & Q9BQL6 & Q7Z7L7 & Q96ME1 & Q9Y2A7 & 043395 \\
\hline P46777 & 043684 & P62249 & P30050 & Q3KQU3 & 095239 \\
\hline P42224 & 095271 & Q9NRF8 & A8MSH5 & K4DI95 & P62995 \\
\hline Q13485 & P49757 & Q9Y295 & 000458 & 075436 & P30101 \\
\hline 060716 & Q9Y3S1 & 060942 & Q9UBK8 & H7BXP1 & 060832 \\
\hline P06748 & P05783 & P19388 & Q8ND56 & Q9BX40 & Q99459 \\
\hline Q13148 & Q9NW82 & Q8NCE2 & G3XAI2 & Q9UPQ0 & P67936 \\
\hline P14635 & E9PBB4 & 095218 & J3KNK4 & Q14CX7 & P13797 \\
\hline E7ENU4 & B7ZKM8 & 094887 & P07355 & Q15056 & P08621 \\
\hline P43487 & Q92900 & P53621 & Q9UPR3 & Q9GZS3 & Q07666 \\
\hline 043196 & P61160 & Q9UBF2 & 015145 & P12956 & P31946 \\
\hline 096018 & Q15370 & Q9UNF1 & Q92620 & Q08AD1 & Q15233 \\
\hline Q9HAP2 & Q5VTI5 & Q6R327 & Q9NSD9 & Q13136 & A8MXP9 \\
\hline Q6NXT1 & Q8NHG8 & P17812 & Q8TAF3 & P42285 & B2RNG4 \\
\hline C9JDG0 & Q6PL24 & Q9HAU5 & P62314 & E9PRY8 & Q92997 \\
\hline Q15654 & Q9UL7 & Q9Y4H2 & Q9UK41 & Q14103 & B5MCU0 \\
\hline 000571 & P27361 & 015514 & Q15061 & B7ZKT7 & Q8NFZ5 \\
\hline Q15942 & 015234 & Q8NE71 & Q9P265 & P78346 & P07197 \\
\hline
\end{tabular}




\begin{tabular}{|c|c|c|c|c|c|}
\hline 015530 & Q15436 & P05388 & Q9NR12 & 060306 & E7EN19 \\
\hline Q02750 & P49674 & Q6P1N0 & Q9Y6W5 & 095747 & E9PAL6 \\
\hline P41743 & Q9Y597 & A5YKK6 & P46783 & P49588 & E9PC69 \\
\hline P49137 & Q676U5 & Q504Q3 & Q9HB21 & $\mathrm{Q9H6H4}$ & E9PF99 \\
\hline Q7Z2W4 & Q13492 & P49792 & Q15054 & P09651 & E9PR30 \\
\hline P26196 & Q9UDY8 & P23193 & Q9P2Y5 & Q13247 & F5GZ78 \\
\hline Q9UDY2 & P49790 & P59998 & Q5VYS8 & Q13151 & F5H604 \\
\hline Q9NUL3 & Q9P107 & Q13371 & Q96SB4 & Q8NCA5 & HOY4E8 \\
\hline P14635 & P68400 & P62851 & F5H2R7 & P02792 & HOYEF7 \\
\hline 000401 & Q5VW36 & Q7Z460 & 043583 & Q7L2E3 & HOYKD8 \\
\hline Q13574 & Q52LW3 & Q5T5C7 & Q9Y4E8 & Q6UXN9 & H3BQK9 \\
\hline 015534 & Q6PD74 & Q96PY6 & 043823 & P22087 & P62068 \\
\hline Q5D1E8 & Q6P2P2 & Q9UJY4 & Q6PGP7 & Q96B97 & J3KРMЗ \\
\hline Q9UI10 & Q9HC44 & J3KP97 & Q68DQ2 & P18754 & K7EM18 \\
\hline A0AV96 & Q14558 & Q9Y6V7 & HOY9Z5 & Q9NZI8 & Q8TBC3 \\
\hline Q9H6K1 & Q15057 & P62269 & Q9UFF9 & Q70J99 & 000507 \\
\hline P47736 & P60866 & P62847 & Q86X10 & Q8TDJ6 & 014907 \\
\hline B4DZH6 & Q92600 & Q5T447 & 015066 & P30622 & 015063 \\
\hline Q9ULI2 & I3L097 & B4DXJ1 & Q9BZF9 & P63104 & 015553 \\
\hline Q8TF05 & P46060 & Q9BY44 & Q8ND04 & B1AK88 & 043900 \\
\hline Q00537 & Q9H4B6 & 043865 & 060318 & MOQZR4 & 060239 \\
\hline Q13546 & Q9UPM8 & 094927 & Q93073 & Q9Y5S9 & POCAP2 \\
\hline Q9Y4K1 & P22681 & 000443 & Q13610 & 075116 & P15170 \\
\hline Q15434 & Q13615 & Q5VZE5 & Q9H074 & Q14847 & P36954 \\
\hline Q8IXW5 & P24928 & Q9BVM2 & P17612 & P35241 & P41279 \\
\hline Q9Y484 & Q8IW35 & Q53EZ4 & B4DYX9 & Q5W0V3 & Q9H1A6 \\
\hline Q00653 & 014617 & Q6ZS17 & P26373 & Q13428 & P62273 \\
\hline Q8WU79 & Q13496 & Q4J6C6 & Q96P48 & P06730 & P62304 \\
\hline Q99567 & P61962 & Q96HN2 & P61981 & 000267 & P62312 \\
\hline Q9BQK8 & Q9BUB5 & P49840 & P19338 & P47914 & P62857 \\
\hline Q5U5Q3 & P53992 & P62263 & Q9UNS2 & Q9UJU6 & P62875 \\
\hline Q6P2H3 & P62487 & Q9HD67 & Q7Z2T5 & Q15185 & P62891 \\
\hline Q13144 & P78337 & Q9UKZ1 & Q8NC51 & Q6GYQ0 & P63167 \\
\hline
\end{tabular}


100

\begin{tabular}{|c|c|c|c|c|c|}
\hline Q8WUX9 & J3KNL6 & P29353 & Q2TAY7 & 095999 & P63220 \\
\hline 075843 & Q9UGP4 & Q99613 & B4DR52 & Q8TD19 & D6R919 \\
\hline Q2TAZO & 043164 & Q9H6SO & P08670 & Q9UBU9 & P84090 \\
\hline Q9H6D7 & Q15652 & P30153 & Q9NW08 & Q9Y5S2 & Q13772 \\
\hline Q8N122 & Q8N8R7 & E9PM46 & P47813 & P15311 & Q14679 \\
\hline C9JC87 & Q13190 & Q8TC07 & F5HFY4 & P05386 & F5GY24 \\
\hline Q8WXG6 & Q8N6H7 & J3KNRO & Q8TD16 & Q93009 & Q15051 \\
\hline Q5JTW2 & Q9H9H4 & Q5VT06 & Q8IUD2 & Q15637 & Q15366 \\
\hline Q96K76 & P52630 & P62280 & Q14527 & Q96118 & Q16204 \\
\hline Q9UNY4 & Q9UMZ2 & Q9H267 & 060333 & 043149 & Q5JTC6 \\
\hline P09132 & 014744 & Q658Y4 & P52907 & 014776 & Q5T2D3 \\
\hline Q8NDI1 & P37198 & Q9H0W8 & Q9NSI2 & P13984 & Q5TC82 \\
\hline 000203 & G5E948 & P25098 & 000178 & Q9BUJ2 & Q5XG87 \\
\hline Q96B36 & Q14671 & Q9Y230 & POCOS8 & Q14978 & Q6P3S1 \\
\hline Q8IX03 & Q86XL3 & Q9Y6K9 & P23921 & Q08945 & Q86UY5 \\
\hline Q96CN4 & Q9BPY3 & P19387 & Q969S3 & P51114 & Q8N2Y8 \\
\hline P49458 & Q9BYV8 & Q96RG2 & G3V0I6 & P52272 & Q8N4B1 \\
\hline P35658 & B4DKT0 & Q7Z4H7 & Q92625 & Q04917 & Q8NDG6 \\
\hline 095684 & P41091 & Q96QR8 & Q12768 & P52701 & Q8NDH2 \\
\hline Q5T7W7 & Q9BSJ2 & Q8NEC7 & Q96HR8 & Q9C0C2 & Q8WVF1 \\
\hline Q93008 & Q9BX10 & P41214 & Q9UBQ5 & 060294 & Q8WZ19 \\
\hline Q8IYB5 & Q9Y6K5 & Q96F86 & Q15650 & P17844 & Q96A73 \\
\hline Q8NDV7 & 094855 & Q5TKA1 & 075937 & P31946 & Q96E09 \\
\hline A7E2V4 & Q14157 & Q13617 & Q86VP6 & Q86UK7 & Q96FQ6 \\
\hline B4E0T2 & Q9BT25 & P61966 & Q7L7X3 & Q9Y3I0 & Q96MX6 \\
\hline P60510 & Q9UQ80 & Q9P1U1 & Q00341 & P26599 & Q96P47 \\
\hline Q9UPU7 & Q9Y3S2 & Q9NZQ3 & Q86XZ4 & Q9NZN8 & Q96QP1 \\
\hline P19838 & P37108 & Q5H9R7 & Q92917 & Q9BZZ5 & Q99584 \\
\hline P40855 & Q9Y217 & 075607 & Q9Y285 & Q6P1J9 & Q99961 \\
\hline 075420 & Q9Y6Y8 & Q86UU1 & B5ME97 & 015027 & Q9BYI3 \\
\hline Q15369 & Q63HR2 & B0QY89 & P84098 & Q9UKD2 & Q9H0K1 \\
\hline Q14674 & P38606 & Q86UU0 & Q9UPY3 & A6NMQ1 & Q9H8N7 \\
\hline P53677 & Q9Y5A9 & 060518 & Q96KG9 & 043143 & Q9H992 \\
\hline
\end{tabular}


101

\begin{tabular}{|c|c|c|c|c|c|}
\hline P13807 & 095721 & Q12905 & Q01970 & Q8TDX7 & Q9HD40 \\
\hline Q9H446 & Q8NFH4 & P07900 & P05387 & H3BLZ8 & Q9NP61 \\
\hline D6REX3 & 094972 & Q9Y5T5 & P23258 & Q96DH6 & Q9NRH1 \\
\hline Q9UHD2 & B4DZD6 & Q14258 & P27695 & B4DWW4 & Q9NTX7 \\
\hline P51617 & P57772 & P62316 & Q68CZ6 & P23284 & Q9NWQ9 \\
\hline Q5SQN1 & Q96AC1 & P62266 & Q9P258 & G3V1L9 & Q9NYY3 \\
\hline Q6UUV9 & Q6PGN9 & Q96ED9 & Q7Z6B7 & Q9Y314 & Q9P1Y5 \\
\hline Q9NYF3 & P48729 & Q9UL18 & Q9NR09 & Q06265 & Q9UH36 \\
\hline Q6AWC2 & Q9H0J9 & 095163 & Q9BZI7 & Q8NBJ5 & Q9UK97 \\
\hline Q8N961 & Q15843 & Q9H0K6 & Q5T6N4 & Q9Y5B9 & Q9ULL1 \\
\hline Q86VV8 & 015143 & 014641 & Q14155 & Q16513 & Q9Y2J4 \\
\hline P47974 & Q6P158 & P62979 & Q13393 & Q14498 & Q9Y333 \\
\hline Q8WUF5 & Q9C0C7 & P61964 & 014920 & O14777 & R4GN35 \\
\hline Q9Y2T2 & С9J6P4 & P62277 & Q9Y5K6 & G8JLB6 & J3KQN4 \\
\hline Q86YR5 & Q9UHW5 & Q9UQN3 & 043172 & P10155 & Q5SRE5 \\
\hline Q9H3F6 & F5HOR1 & 095714 & P15880 & 043809 & P62888 \\
\hline Q9H6R7 & Q8IV48 & B8ZZ87 & P62753 & E7ES43 & Q9UHR6 \\
\hline Q9P2E3 & Q96A49 & P41240 & Q00839 & Q9Y613 & Q5T1Z8 \\
\hline B9EGP5 & Q99081 & Q9Y265 & Q9UH62 & Q92541 & Q8TCG1 \\
\hline Q9Y446 & B4DNJ6 & Q96CS2 & Q5F1R6 & 095456 & Q93052 \\
\hline P46976 & P62136 & A8MXB7 & Q01105 & Q7KZF4 & Q9UBB6 \\
\hline Q9UKE5 & Q15025 & Q9Y6B7 & 014802 & P30260 & Q9BRP1 \\
\hline Q58A45 & P62854 & 014579 & Q641Q2 & Q9Y520 & C9JDG0 \\
\hline Q2M2I8 & J3KPL5 & Q63ZY3 & Q6P0Q8 & Q9UKY7 & P49207 \\
\hline Q68DC2 & 060566 & P46781 & Q8WWM7 & С9K060 & Q15654 \\
\hline P52298 & Q5JTD0 & BOYIW6 & P46782 & Q01085 & Q2TAM5 \\
\hline Q9BYJ9 & Q96EY5 & Q5SRQ6 & Q92574 & Q6ZSJ8 & 000571 \\
\hline P36507 & P62714 & Q14008 & P53999 & 095155 & P57678 \\
\hline Q70E73 & P11908 & P56377 & P61313 & P28290 & Q96D46 \\
\hline Q96FC9 & 076094 & Q8TF46 & Q96EV2 & P35658 & Q8N1F7 \\
\hline Q9H977 & Q9H425 & P52306 & F8VQ10 & Q00403 & Q9NRX1 \\
\hline Q6UUV7 & 043572 & Q96AZ6 & 060784 & Q9HB71 & Q15942 \\
\hline P11532 & Q5VV41 & P61221 & P02794 & Q9NVP2 & P30876 \\
\hline
\end{tabular}




\begin{tabular}{|c|c|c|c|c|c|}
\hline Q9P0J7 & Q7L2H7 & 060231 & P49005 & Q9Y294 & P18077 \\
\hline 095486 & Q8N8S7 & P42766 & P08579 & P35637 & Q9UHI6 \\
\hline Q7Z309 & Q99816 & P36873 & Q00577 & Q01130 & Q9BVS4 \\
\hline Q8TEU7 & Q5T4S7 & Q5VZK9 & 015160 & Q86TU7 & Q13501 \\
\hline Q684P5 & Q9Y4G8 & Q9Y3A5 & Q9Y6I3 & 075165 & Q13895 \\
\hline P49770 & 000743 & P52594 & Q99543 & Q16626 & Q13310 \\
\hline F8WEA9 & M0R2B7 & Q92499 & Q8N1G2 & P63279 & P63151 \\
\hline 043524 & Q9H7P9 & Q8NFH3 & 000139 & 015347 & Q9UPW5 \\
\hline Q92539 & P50548 & Q9Y4B6 & Q86US8 & B4DUT8 & Q6Y7W6 \\
\hline J3KNEO & P55010 & 060256 & Q99707 & Q13442 & 060573 \\
\hline Q9ULX3 & P62244 & F5H527 & P07951 & 094888 & Q9UJX4 \\
\hline Q96FK6 & Q92540 & P08865 & P54577 & Q9BVP2 & Q14247 \\
\hline Q9BVC4 & Q66LE6 & Q9Y450 & 043633 & Q53GS9 & Q6NW29 \\
\hline Q9UPN7 & 094804 & E7EX17 & Q96J02 & Q99729 & P39023 \\
\hline Q9NR50 & P61158 & 015357 & P46063 & Q16851 & P11940 \\
\hline 095905 & Q8NEB9 & Q9H2U1 & 000562 & Q9H4M9 & Q12769 \\
\hline Q15437 & Q9NTZ6 & Q2NKX8 & 060506 & P27348 & Q2NL82 \\
\hline Q01968 & Q8IWV8 & 015371 & Q6PKG0 & Q7Z3B4 & P62913 \\
\hline Q6BDS2 & Q15047 & Q14152 & 075822 & P61978 & Q02543 \\
\hline Q53ET0 & 075688 & 075821 & Q8N4C8 & 060749 & P61254 \\
\hline P04049 & Q6ULP2 & Q00536 & I3L504 & Q9UQE7 & Q9BRP8 \\
\hline A4D1P6 & Q8N6T3 & Q14320 & P40818 & Q13325 & P36578 \\
\hline P50552 & Q8TEQ6 & Q5VY93 & Q8TCY9 & Q8IWC1 & Q9NVN8 \\
\hline Q9NT62 & 075818 & P14735 & P35573 & P78537 & P62899 \\
\hline Q86SQ0 & Q9BRX2 & P85037 & F5H1X8 & Q9NW13 & Q13451 \\
\hline Q9H000 & P68104 & 060524 & Q9BPZ7 & P61764 & Q9H3S7 \\
\hline Q14232 & P20042 & P60891 & Q8IYB7 & F8WCP6 & 015294 \\
\hline Q9NWT6 & F5H874 & P61353 & P10644 & P78527 & P41743 \\
\hline Q2M3G4 & Q9UM82 & P35250 & Q96P16 & P07910 & Q96GA3 \\
\hline Q9BVQ7 & Q3V6T2 & Q9H9G7 & Q96PZO & K7ELC2 & Q9BWH6 \\
\hline Q15468 & Q12899 & Q07960 & 000541 & Q86V48 & Q12800 \\
\hline Q13829 & P60842 & Q3MHD2 & Q5GLZ8 & Q5RKV6 & Q9UI30 \\
\hline Q7L3B6 & Q9UK59 & H7C107 & Q9NUP1 & P31942 & Q5T6F2 \\
\hline
\end{tabular}




\begin{tabular}{|c|c|c|c|c|c|}
\hline Q6P3W7 & B3KXW5 & Q9BW27 & Q15477 & Q8WXX5 & Q8N6R0 \\
\hline P49841 & 015144 & Q13616 & Q5SW79 & P55072 & Q9H8Y5 \\
\hline Q04864 & A3KN83 & P62750 & Q9Y6A4 & P84103 & P50914 \\
\hline Q96IF1 & 014730 & Q10567 & Q8WX93 & H7BZT4 & Q9BZE4 \\
\hline Q96EN8 & Q8WVM8 & Q9Y678 & 015511 & B4DVB8 & Q6VY07 \\
\hline J3КPC8 & Q9ULC4 & Q9BPX5 & Q9H6U6 & P26583 & Q9H1A4 \\
\hline Q9UPQ9 & E9PB12 & Q9HCE1 & Q12906 & Q9H7D0 & Q9BV44 \\
\hline 000750 & Q6P2E9 & P23396 & Q9P031 & P09429 & P62917 \\
\hline B3KS98 & P62140 & Q96P11 & Q53EL6 & D6W592 & P40429 \\
\hline P78406 & Q562E7 & Q66K74 & Q13618 & Q99575 & 000488 \\
\hline P32121 & Q9UPQ3 & Q9NVM6 & Q9NZB2 & Q9Y2W2 & P62906 \\
\hline P05198 & 060504 & Q9NZT2 & 075175 & J3KQ32 & P61247 \\
\hline Q9UKV8 & $\mathrm{F} 5 \mathrm{H} 2 \mathrm{M} 7$ & Q96S55 & Q9UIV1 & P38432 & Q9BRJ6 \\
\hline Q8TEH3 & B4DGT8 & Q75MJ1 & Q08J23 & 075663 & J3QQ67 \\
\hline Q8NB90 & B8ZZN6 & Q147X3 & P27635 & P11021 & P18124 \\
\hline Q8TB45 & 095628 & B7WPE2 & 043670 & Q13283 & P46776 \\
\hline Q969Q6 & $\mathrm{Q} 8 \mathrm{IZH} 2$ & Q8IXQ3 & Q14181 & P22694 & Q96CT7 \\
\hline QOJRZ9 & P62495 & Q7Z2Z2 & 095707 & Q92878 & P32969 \\
\hline Q9NUU7 & Q15020 & Q9NYL2 & Q92979 & Q96PK6 & P52292 \\
\hline 000442 & P63244 & Q8N1G4 & Q6RFH5 & P33991 & Q02878 \\
\hline G3V3G9 & B3KSH1 & Q14677 & B4EOY9 & P49643 & 000505 \\
\hline B4DR47 & P53990 & P05455 & P62258 & Q04323 & F8W727 \\
\hline Q5PRF9 & Q9GZZ9 & P78344 & P13010 & 075717 & P46778 \\
\hline Q9NRY5 & P55884 & Q8WU90 & Q13162 & P22626 & Q8N6M0 \\
\hline 060841 & B4DQM4 & P35658 & P42694 & Q16630 & P56537 \\
\hline Q13287 & Q9H6T3 & P38935 & QOVDF9 & Q9UKK3 & 000629 \\
\hline Q9H1Y0 & P30154 & Q14C86 & Q9NQT8 & Q16629 & P18621 \\
\hline Q68CZ2 & P13639 & P39748 & P08238 & Q7Z417 & Q9UJX2 \\
\hline P61011 & G5E9Q2 & MoQZW1 & Q9NW64 & P43246 & Q9BQ67 \\
\hline Q8IZW8 & Q7Z478 & Q8IWZ3 & P30419 & P31327 & Q9Y2L1 \\
\hline P28482 & Q9BXS5 & Q2KHT3 & Q9C0C9 & Q9NUQ3 & Q9H7E9 \\
\hline P52948 & Q9UET6 & Q8N3C0 & Q9UMY1 & Q9NYF8 & P61201 \\
\hline Q8WWN8 & Q9NX04 & Q96JN8 & P33992 & Q16543 & Q7L5N1 \\
\hline
\end{tabular}




\begin{tabular}{|l|l|l|l|l|l|}
\hline Q3YEC7 & Q09161 & G3V4K3 & Q14444 & Q9NPD8 & P14635 \\
\hline Q9C0D3 & Q9NP16 & P62701 & O00425 & H3BSH7 & P43487 \\
\hline Q9H6S3 & Q15036 & Q04637 & O75179 & Q14738 & P61513 \\
\hline Q92747 & H0YLI7 & P08708 & Q14683 & Q9BQ70 & Q99873 \\
\hline Q6ZRV2 & Q9P2D3 & P62310 & E7EVA0 & Q9UN86 & \\
\hline Q9NWS0 & Q14244 & Q9P2D0 & P20810 & P39687 & \\
\hline O15111 & P35606 & P40938 & O15061 & O43432 & \\
\hline Q9H814 & P38919 & Q96H20 & Q08211 & E9PB61 & \\
\hline
\end{tabular}

Table 12-4 List of Uniprot IDs of proteins from MS analysis

12.2.4 321 Proteins with a Predicted NES

\begin{tabular}{|l|l|l|l|l|l|}
\hline Q6UUV9 & O95684 & Q5VTI5 & Q13371 & Q9UNS2 & Q16513 \\
\hline P47974 & Q8IYB5 & Q9UL7 & P62851 & Q8TD16 & Q9Y613 \\
\hline Q53ET0 & Q8NDV7 & O15234 & Q7Z460 & Q8IUD2 & P28290 \\
\hline P04049 & Q9UPU7 & Q13492 & Q5T5C7 & Q14527 & Q16626 \\
\hline Q6P3W7 & P40855 & Q9P107 & J3KP97 & O60333 & Q9UQE7 \\
\hline Q86XL3 & Q14674 & P60866 & Q9BY44 & Q9NS12 & P61764 \\
\hline B4DZD6 & Q9H446 & Q13615 & O00443 & G3V016 & K7ELC2 \\
\hline Q9Y450 & Q5SQN1 & P24928 & Q99613 & O75937 & Q86V48 \\
\hline Q00536 & Q8N961 & Q8IW35 & J3KNR0 & Q86XZ4 & J3KQ32 \\
\hline Q8N1G4 & Q8WUF5 & Q9BUB5 & Q5VT06 & P84098 & P11021 \\
\hline Q8IW73 & Q9P2E3 & P53992 & P25098 & Q9P258 & Q92878 \\
\hline H0Y9Z5 & B9EGP5 & Q15652 & Q8NEC7 & Q5T6N4 & E9PB61 \\
\hline Q9NR09 & Q9UKE5 & P52630 & P41214 & Q5F1R6 & E9PAV3 \\
\hline O43633 & Q58A45 & Q14671 & Q13617 & Q641Q2 & J3KN67 \\
\hline P26358 & Q68DC2 & B4DKT0 & Q5H9R7 & Q6P0Q8 & Q15691 \\
\hline Q8NDH2 & Q9BYJ9 & Q9BSJ2 & Q86UU1 & P46782 & P67936 \\
\hline P35869 & P36507 & O94855 & Q86UU0 & Q92574 & E9PC69 \\
\hline Q86TB9 & Q9P0J7 & Q9Y5A9 & P07900 & Q96EV2 & E9PF99 \\
\hline Q96D46 & O95486 & O95721 & O95163 & O60784 & F5G278 \\
\hline Q8IXJ6 & Q8TEU7 & P57772 & Q9UQN3 & Q86US8 & F5H604 \\
\hline Q14653 & Q684P5 & Q96AC1 & Q96CS2 & P07951 & H0Y4E8 \\
\hline P25963 & O43524 & O15143 & P52306 & P54577 & H0YEF7 \\
\hline
\end{tabular}


105

\begin{tabular}{|c|c|c|c|c|c|}
\hline P30291 & J3KNEO & Q6P158 & 060231 & P46063 & H3BQK9 \\
\hline P25054 & Q9ULX3 & Q96A49 & F5H527 & Q8TCY9 & 015063 \\
\hline Q9NRA8 & Q96FK6 & P62136 & Q2NKX8 & P35573 & 060239 \\
\hline Q9NQS1 & Q6BDS2 & Q15025 & 075821 & F5H1X8 & P15170 \\
\hline 096013 & Q9NT62 & J3KPL5 & P14735 & Q8IYB7 & Q14679 \\
\hline P42224 & Q86SQ0 & Q96EY5 & 060524 & 075175 & Q15051 \\
\hline P14635 & Q14232 & P11908 & P60891 & B4EOY9 & Q15366 \\
\hline E7ENU4 & Q13829 & Q5VV41 & Q10567 & Q9C0C9 & Q5T2D3 \\
\hline Q6NXT1 & J3KPC8 & Q5T4S7 & Q9BPX5 & Q14683 & Q6P3S1 \\
\hline C9JDG0 & Q9UPQ9 & 094804 & Q9NVM6 & 015061 & Q8WVF1 \\
\hline 000571 & Q8TEH3 & 075688 & Q7Z2Z2 & Q08211 & Q96A73 \\
\hline Q02750 & Q8NB90 & Q9UM82 & Q9NYL2 & Q8N163 & Q96QP1 \\
\hline 000401 & Q0JRZ9 & Q3V6T2 & Q8WU90 & Q00688 & Q9H0K1 \\
\hline 015534 & G3V3G9 & P60842 & P38935 & K4DI95 & Q9H8N7 \\
\hline B4DZH6 & B4DR47 & B3KXW5 & Q8N3C0 & Q08AD1 & Q9HD40 \\
\hline Q9ULI2 & Q9NRY5 & Q562E7 & Q9P2D0 & Q13136 & Q9NTX7 \\
\hline Q00537 & 060841 & Q9UPQ3 & P62081 & P42285 & Q9Y2J4 \\
\hline Q13546 & Q68CZ2 & $\mathrm{F} 5 \mathrm{H} 2 \mathrm{M} 7$ & 000458 & E9PRY8 & R4GN35 \\
\hline Q15434 & P52948 & B4DGT8 & Q8ND56 & B7ZKT7 & Q5T1Z8 \\
\hline Q00653 & Q8WWN8 & 095628 & G3XAI2 & Q13151 & Q9UBB6 \\
\hline Q5U5Q3 & Q92747 & Q8IZH2 & Q9UPR3 & P02792 & Q13501 \\
\hline Q6P2H3 & Q9C0B9 & B4DQM4 & Q92620 & Q8TDJ6 & Q9UPW5 \\
\hline Q13144 & Q7Z739 & Q15036 & Q15061 & P30622 & Q9BRP8 \\
\hline Q8WUX9 & Q96PU5 & P35606 & Q9P265 & M0QZR4 & Q9NVN8 \\
\hline Q2TAZO & 043933 & P53618 & Q9P2Y5 & 075116 & Q9H3S7 \\
\hline Q8N122 & Q9BQL6 & P46934 & 043823 & Q5W0V3 & Q96GA3 \\
\hline Q9UNY4 & P05783 & B3KYA7 & Q6PGP7 & Q9Y5S2 & Q8N6R0 \\
\hline 000203 & Q9NW82 & Q9HAU5 & Q68DQ2 & P15311 & Q9H8Y5 \\
\hline Q96B36 & E9PBB4 & Q8NE71 & Q9BZF9 & Q93009 & Q9BZE4 \\
\hline Q81X03 & B7ZKM8 & Q6P1N0 & 060318 & P52272 & Q9H1A4 \\
\hline Q96CN4 & Q92900 & P49792 & Q13610 & B4DWW4 & J3QQ67 \\
\hline Q9H7E9 & & & & & \\
\hline
\end{tabular}

Table 12-5 321 Uniprot IDs of proteins with a predicted NES 


\section{REFERENCES}

Adachi Y, Yanagida M (1989) Higher order chromosome structure is affected by cold-sensitive mutations in a Schizosaccharomyces pombe gene crm1+ which encodes a 115-kD protein preferentially localized in the nucleus and its periphery. J Cell Biol, 108: 1195-1207

Alavian CN, Politz JC, Lewandowski LB, Powers CM, Pederson T (2004) Nuclear export of signal recognition particle RNA in mammalian cells. Biochem Biophys Res Commun, 313: 351-355

Alberts B, Johnson A, Lewis J, Raff M, Roberts K, Walter P. (2007) Molecular Biology of the Cell. Garland Science

Andrade MA, Bork P (1995) HEAT repeats in the Huntington's disease protein. Nat Genet, 11: 115-116

Arnold M, Nath A, Wohlwend D, Kehlenbach RH (2006) Transportin is a major nuclear import receptor for cFos: a novel mode of cargo interaction. J Biol Chem, 281: 5492-5499

Ashburner M, Ball CA, Blake JA, Botstein D, Butler H, Cherry JM, Davis AP, Dolinski K, Dwight SS, Eppig JT, Harris MA, Hill DP, Issel-Tarver L, Kasarskis A, Lewis S, Matese JC, Richardson JE, Ringwald M, Rubin GM, Sherlock G (2000) Gene ontology: tool for the unification of biology. The Gene Ontology Consortium. Nat Genet, 25: 25-29

Au S, Pante N (2012) Nuclear transport of baculovirus: revealing the nuclear pore complex passage. J Struct Biol, 177: 90-98

Bayliss R, Littlewood T, Stewart M (2000) Structural basis for the interaction between FxFG nucleoporin repeats and importin-beta in nuclear trafficking. Cell, 102: 99-108

Bayliss R, Ribbeck K, Akin D, Kent HM, Feldherr CM, Gorlich D, Stewart M (1999) Interaction between NTF2 and XFxFG-containing nucleoporins is required to mediate nuclear import of RanGDP. J Mol Biol, 293: 579-593

Beckett D, Kovaleva E, Schatz PJ (1999) A minimal peptide substrate in biotin holoenzyme synthetasecatalyzed biotinylation. Protein Sci, 8: 921-929

Ben-Shem A, Garreau de Loubresse N, Melnikov S, Jenner L, Yusupova G, Yusupov M (2011) The structure of the eukaryotic ribosome at 3.0 A resolution. Science, 334: 1524-1529

Bilokapic S, Schwartz TU (2012) 3D ultrastructure of the nuclear pore complex. Curr Opin Cell Biol, 24: 86-91

Bischoff FR, Klebe C, Kretschmer J, Wittinghofer A, Ponstingl H (1994) RanGAP1 induces GTPase activity of nuclear Ras-related Ran. Proceedings of the National Academy of Sciences of the United States of America, 91: 2587-2591

Bischoff FR, Gorlich D (1997) RanBP1 is crucial for the release of RanGTP from importin beta-related nuclear transport factors. FEBS Lett, 419: 249-254

Bischoff FR, Krebber H, Smirnova E, Dong W, Ponstingl H (1995) Co-activation of RanGTPase and inhibition of GTP dissociation by Ran-GTP binding protein RanBP1. EMBO J, 14: 705-715

Bischoff FR, Ponstingl H (1991) Catalysis of guanine nucleotide exchange on Ran by the mitotic regulator RCC1. Nature, 354: 80-82

Bogerd HP, Fridell RA, Benson RE, Hua J, Cullen BR (1996) Protein sequence requirements for function of the human T-cell leukemia virus type 1 Rex nuclear export signal delineated by a novel in vivo randomization-selection assay. Molecular and cellular biology, 16: 4207-4214

Bohnsack MT, Czaplinski K, Gorlich D (2004) Exportin 5 is a RanGTP-dependent dsRNA-binding protein that mediates nuclear export of pre-miRNAs. RNA, 10: 185-191 
Bohnsack MT, Regener K, Schwappach B, Saffrich R, Paraskeva E, Hartmann E, Gorlich D (2002) Exp5 exports eEF1A via tRNA from nuclei and synergizes with other transport pathways to confine translation to the cytoplasm. EMBO J, 21: 6205-6215

Bonner WM (1975) Protein migration into nuclei. II. Frog oocyte nuclei accumulate a class of microinjected oocyte nuclear proteins and exclude a class of microinjected oocyte cytoplasmic proteins. J Cell Biol, 64: $431-437$

Brownawell AM, Macara IG (2002) Exportin-5, a novel karyopherin, mediates nuclear export of doublestranded RNA binding proteins. J Cell Biol, 156: 53-64

Brunet A, Kanai F, Stehn J, Xu J, Sarbassova D, Frangioni JV, Dalal SN, DeCaprio JA, Greenberg ME, Yaffe MB (2002) 14-3-3 transits to the nucleus and participates in dynamic nucleocytoplasmic transport. J Cell Biol, 156: 817-828

Bullock TL, Clarkson WD, Kent HM, Stewart M (1996) The 1.6 angstroms resolution crystal structure of nuclear transport factor 2 (NTF2). J Mol Biol, 260: 422-431

Chang C-C, Lin C-J (2001) LIBSVM: A library for support vector machines.

Chook YM, Blobel G (1999) Structure of the nuclear transport complex karyopherin-beta2-Ran x GppNHp. Nature, 399: 230-237

Chuderland D, Konson A, Seger R (2008) Identification and characterization of a general nuclear translocation signal in signaling proteins. Mol Cell, 31: 850-861

Cingolani G, Petosa C, Weis K, Muller CW (1999) Structure of importin-beta bound to the IBB domain of importin-alpha. Nature, 399: 221-229

Cock PJ, Antao T, Chang JT, Chapman BA, Cox CJ, Dalke A, Friedberg I, Hamelryck T, Kauff F, Wilczynski B, de Hoon MJ (2009) Biopython: freely available Python tools for computational molecular biology and bioinformatics. Bioinformatics, 25: 1422-1423

Colwell LJ, Brenner MP, Ribbeck K (2010) Charge as a selection criterion for translocation through the nuclear pore complex. PLoS Comput Biol, 6: e1000747

Craig E, Zhang ZK, Davies KP, Kalpana GV (2002) A masked NES in INI1/hSNF5 mediates hCRM1-dependent nuclear export: implications for tumorigenesis. EMBO J, 21: 31-42

Cullen BR (1992) Mechanism of action of regulatory proteins encoded by complex retroviruses. Microbiol Rev, 56: 375-394

Dean KA, von Ahsen O, Gorlich D, Fried HM (2001) Signal recognition particle protein 19 is imported into the nucleus by importin 8 (RanBP8) and transportin. J Cell Sci, 114: 3479-3485

Delphis A, Beman A, Batavorum L (1719) Leeuwenhoek, A. van: Opera Omnia, seu Arcana Naturae ope exactissimorum Microscopiorum detecta, experimentis variis comprobata. Epistolis ad varios illustres viros $J$,

Denning DP, Patel SS, Uversky V, Fink AL, Rexach M (2003) Disorder in the nuclear pore complex: the FG repeat regions of nucleoporins are natively unfolded. Proc Natl Acad Sci U S A, 100: 2450-2455

Dian C, Bernaudat F, Langer K, Oliva MF, Fornerod M, Schoehn G, Muller CW, Petosa C (2013) Structure of a Truncation Mutant of the Nuclear Export Factor CRM1 Provides Insights into the Auto-Inhibitory Role of Its C-Terminal Helix. Structure, 21: 1338-1349

Dinkel H, Michael S, Weatheritt RJ, Davey NE, Van Roey K, Altenberg B, Toedt G, Uyar B, Seiler M, Budd A, Jodicke L, Dammert MA, Schroeter C, Hammer M, Schmidt T, Jehl P, McGuigan C, Dymecka M, Chica C, Luck K, Via A, Chatr-Aryamontri A, Haslam N, Grebnev G, Edwards RJ, Steinmetz MO, Meiselbach 
H, Diella F, Gibson TJ (2012) ELM--the database of eukaryotic linear motifs. Nucleic Acids Res, 40: D242-51

Dong X, Biswas A, Suel KE, Jackson LK, Martinez R, Gu H, Chook YM (2009) Structural basis for leucine-rich nuclear export signal recognition by CRM1. Nature, 458: 1136-1141

Drivas GT, Shih A, Coutavas E, Rush MG, D'Eustachio P (1990) Characterization of four novel ras-like genes expressed in a human teratocarcinoma cell line. Mol Cell Biol, 10: 1793-1798

Engel K, Kotlyarov A, Gaestel M (1998) Leptomycin B-sensitive nuclear export of MAPKAP kinase 2 is regulated by phosphorylation. EMBO J, 17: 3363-3371

Engelsma D, Bernad R, Calafat J, Fornerod M (2004) Supraphysiological nuclear export signals bind CRM1 independently of RanGTP and arrest at Nup358. EMBO J, 23: 3643-3652

Engler C, Kandzia R, Marillonnet S (2008) A one pot, one step, precision cloning method with high throughput capability. PLoS One, 3: e3647

Englmeier L, Olivo JC, Mattaj IW (1999) Receptor-mediated substrate translocation through the nuclear pore complex without nucleotide triphosphate hydrolysis. Curr Biol, 9: 30-41

Falcon S, Gentleman R (2007) Using GOstats to test gene lists for GO term association. Bioinformatics, 23: 257-258

Fantozzi DA, Harootunian AT, Wen W, Taylor SS, Feramisco JR, Tsien RY, Meinkoth JL (1994) Thermostable inhibitor of CAMP-dependent protein kinase enhances the rate of export of the kinase catalytic subunit from the nucleus. J Biol Chem, 269: 2676-2686

Feng W, Benko AL, Lee JH, Stanford DR, Hopper AK (1999) Antagonistic effects of NES and NLS motifs determine S. cerevisiae Rna1p subcellular distribution. Journal of cell science, 112: 339-347

Fischer U, Huber J, Boelens WC, Mattaj IW, Luhrmann R (1995) The HIV-1 Rev activation domain is a nuclear export signal that accesses an export pathway used by specific cellular RNAs. Cell, 82: 475-483

Fornerod M, Ohno M, Yoshida M, Mattaj IW (1997) CRM1 is an export receptor for leucine-rich nuclear export signals. Cell, 90: 1051-1060

Franke WW, Scheer U (1974) Pathways of nucleocytoplasmic translocation of ribonucleoproteins. Symp Soc Exp Biol, 249-282

Frey S, Gorlich D (2007) A saturated FG-repeat hydrogel can reproduce the permeability properties of nuclear pore complexes. Cell, 130: 512-523

Frey S, Gorlich D (2009) FG/FxFG as well as GLFG repeats form a selective permeability barrier with selfhealing properties. $E M B O \mathrm{~J}, 28:$ 2554-2567

Frey S, Richter RP, Gorlich D (2006) FG-rich repeats of nuclear pore proteins form a three-dimensional meshwork with hydrogel-like properties. Science, 314: 815-817

Fridell RA, Fischer U, Luhrmann R, Meyer BE, Meinkoth JL, Malim MH, Cullen BR (1996) Amphibian transcription factor IIIA proteins contain a sequence element functionally equivalent to the nuclear export signal of human immunodeficiency virus type 1 Rev. Proc Natl Acad Sci U S A, 93: 2936-2940

Fu SC, Imai K, Horton P (2011) Prediction of leucine-rich nuclear export signal containing proteins with NESsential. Nucleic acids research, 39: e111-e111

Fukuda M, Asano S, Nakamura T, Adachi M, Yoshida M, Yanagida M, Nishida E (1997) CRM1 is responsible for intracellular transport mediated by the nuclear export signal. Nature, 390: 308-311

Fukuda M, Gotoh I, Gotoh Y, Nishida E (1996) Cytoplasmic localization of mitogen-activated protein kinase kinase directed by its $\mathrm{NH2}$-terminal, leucine-rich short amino acid sequence, which acts as a nuclear export signal. J Biol Chem, 271: 20024-20028 
Fuxreiter M, Tompa P, Simon I (2007) Local structural disorder imparts plasticity on linear motifs. Bioinformatics, 23: 950-956

Gareau JR, Reverter D, Lima CD (2012) Determinants of small ubiquitin-like modifier 1 (SUMO1) protein specificity, E3 ligase, and SUMO-RanGAP1 binding activities of nucleoporin RanBP2. J Biol Chem, 287: 4740-4751

Gerace L (1995) Nuclear export signals and the fast track to the cytoplasm. Cell, 82: 341-344

Gontan C, Guttler T, Engelen E, Demmers J, Fornerod M, Grosveld FG, Tibboel D, Gorlich D, Poot RA, Rottier RJ (2009) Exportin 4 mediates a novel nuclear import pathway for Sox family transcription factors. $J$ Cell Biol, 185: 27-34

Gorlich D, Dabrowski M, Bischoff FR, Kutay U, Bork P, Hartmann E, Prehn S, Izaurralde E (1997) A novel class of RanGTP binding proteins. J Cell Biol, 138: 65-80

Gorlich D, Kostka S, Kraft R, Dingwall C, Laskey RA, Hartmann E, Prehn S (1995) Two different subunits of importin cooperate to recognize nuclear localization signals and bind them to the nuclear envelope. Curr Biol, 5: 383-392

Gorlich D, Kutay U (1999) Transport between the cell nucleus and the cytoplasm. Annu Rev Cell Dev Biol, 15: $607-660$

Gorlich D, Mattaj IW (1996) Nucleocytoplasmic transport. Science, 271: 1513-1518

Gorlich D, Pante N, Kutay U, Aebi U, Bischoff FR (1996) Identification of different roles for RanGDP and RanGTP in nuclear protein import. EMBO J, 15: 5584-5594

Gorlich D, Prehn S, Laskey RA, Hartmann E (1994) Isolation of a protein that is essential for the first step of nuclear protein import. Cell, 79: 767-778

Gorlich D, Seewald MJ, Ribbeck K (2003) Characterization of Ran-driven cargo transport and the RanGTPase system by kinetic measurements and computer simulation. EMBO J, 22: 1088-1100

Güttler T, Görlich D (2011) Ran-dependent nuclear export mediators: a structural perspective. The EMBO journal, 30: 3457-3474

Güttler T, Madl T, Neumann P, Deichsel D, Corsini L, Monecke T, Ficner R, Sattler M, Görlich D (2010) NES consensus redefined by structures of PKI-type and Rev-type nuclear export signals bound to CRM1. Nature structural \&amp; molecular biology, 17: 1367-1376

Harootunian AT, Adams SR, Wen W, Meinkoth JL, Taylor SS, Tsien RY (1993) Movement of the free catalytic subunit of cAMP-dependent protein kinase into and out of the nucleus can be explained by diffusion. Mol Biol Cell, 4: 993-1002

Heerklotz D, Döring P, Bonzelius F, Winkelhaus S, Nover L (2001) The balance of nuclear import and export determines the intracellular distribution and function of tomato heat stress transcription factor HsfA2. Molecular and cellular biology, 21: 1759-1768

Henderson BR (2000) Nuclear-cytoplasmic shuttling of APC regulates beta-catenin subcellular localization and turnover. Nat Cell Biol, 2: 653-660

Hillig RC, Renault L, Vetter IR, Drell T, Wittinghofer A, Becker J (1999) The crystal structure of rna1p: a new fold for a GTPase-activating protein. Molecular cell, 3: 781-791

Hopper AK, Traglia HM, Dunst RW (1990) The yeast RNA1 gene product necessary for RNA processing is located in the cytosol and apparently excluded from the nucleus. J Cell Biol, 111: 309-321

Huang TT, Kudo N, Yoshida M, Miyamoto S (2000) A nuclear export signal in the N-terminal regulatory domain of IkappaBalpha controls cytoplasmic localization of inactive NF-kappaB/lkappaBalpha complexes. Proc Natl Acad Sci U S A, 97: 1014-1019 
Huber J, Cronshagen U, Kadokura M, Marshallsay C, Wada T, Sekine M, Luhrmann R (1998a) Snurportin1, an m3G-cap-specific nuclear import receptor with a novel domain structure. EMBO J, 17: 4114-4126

Huber J, Cronshagen U, Kadokura M, Marshallsay C, Wada T, Sekine M, Luhrmann R (1998b) Snurportin1, an m3G-cap-specific nuclear import receptor with a novel domain structure. EMBO J, 17: 4114-4126

Hutchison CAr, Phillips S, Edgell MH, Gillam S, Jahnke P, Smith M (1978) Mutagenesis at a specific position in a DNA sequence. J Biol Chem, 253: 6551-6560

lovine MK, Watkins JL, Wente SR (1995) The GLFG repetitive region of the nucleoporin Nup116p interacts with Kap95p, an essential yeast nuclear import factor. J Cell Biol, 131: 1699-1713

Izaurralde E, Mattaj IW (1995) RNA export. Cell, 81: 153-159

Jakel S, Albig W, Kutay U, Bischoff FR, Schwamborn K, Doenecke D, Gorlich D (1999) The importin beta/importin 7 heterodimer is a functional nuclear import receptor for histone H1. EMBO J, 18: 2411-2423

Jakel S, Gorlich D (1998) Importin beta, transportin, RanBP5 and RanBP7 mediate nuclear import of ribosomal proteins in mammalian cells. EMBO J, 17: 4491-4502

Jakel S, Mingot JM, Schwarzmaier P, Hartmann E, Gorlich D (2002) Importins fulfil a dual function as nuclear import receptors and cytoplasmic chaperones for exposed basic domains. EMBO J, 21: 377-386

Johnson C, Van Antwerp D, Hope TJ (1999) An N-terminal nuclear export signal is required for the nucleocytoplasmic shuttling of IkappaBalpha. EMBO J, 18: 6682-6693

Kabsch W, Mannherz HG, Suck D, Pai EF, Holmes KC (1990) Atomic structure of the actin:DNase I complex. Nature, 347: 37-44

Kaffman A, O'Shea EK (1999) Regulation of nuclear localization: a key to a door. Annu Rev Cell Dev Biol, 15: 291-339

Kahle J, Baake M, Doenecke D, Albig W (2005) Subunits of the heterotrimeric transcription factor NF-Y are imported into the nucleus by distinct pathways involving importin beta and importin 13. Mol Cell Biol, 25: 5339-5354

Kahle J, Piaia E, Neimanis S, Meisterernst M, Doenecke D (2009) Regulation of nuclear import and export of negative cofactor 2. J Biol Chem, 284: 9382-9393

Kalland KH, Szilvay AM, Langhoff E, Haukenes G (1994) Subcellular distribution of human immunodeficiency virus type 1 Rev and colocalization of Rev with RNA splicing factors in a speckled pattern in the nucleoplasm. J Virol, 68: 1475-1485

Kanehisa M (2013) Molecular network analysis of diseases and drugs in KEGG. Methods Mol Biol, 939: 263275

Kanehisa M, Goto S (2000) KEGG: kyoto encyclopedia of genes and genomes. Nucleic Acids Res, 28: 27-30

Kataoka N, Bachorik JL, Dreyfuss G (1999) Transportin-SR, a nuclear import receptor for SR proteins. J Cell Biol, 145: 1145-1152

Kobayashi T, Kamitani W, Zhang G, Watanabe M, Tomonaga K, Ikuta K (2001) Borna disease virus nucleoprotein requires both nuclear localization and export activities for viral nucleocytoplasmic shuttling. J Virol, 75: 3404-3412

Kose S, Imamoto N, Tachibana T, Shimamoto T, Yoneda Y (1997) Ran-unassisted nuclear migration of a 97kD component of nuclear pore-targeting complex. J Cell Biol, 139: 841-849

Kosugi S, Hasebe M, Tomita M, Yanagawa H (2008) Nuclear Export Signal Consensus Sequences Defined Using a Localization-Based Yeast Selection System. Traffic (Copenhagen, Denmark), 9: 2053-2062 
Koyama M, Matsuura Y (2010) An allosteric mechanism to displace nuclear export cargo from CRM1 and RanGTP by RanBP1. EMBO J, 29: 2002-2013

Kudo N, Matsumori N, Taoka H, Fujiwara D, Schreiner EP, Wolff B, Yoshida M, Horinouchi S (1999a) Leptomycin B inactivates CRM1/exportin 1 by covalent modification at a cysteine residue in the central conserved region. Proc Natl Acad Sci U S A, 96: 9112-9117

Kudo N, Taoka H, Toda T, Yoshida M, Horinouchi S (1999b) A novel nuclear export signal sensitive to oxidative stress in the fission yeast transcription factor Pap1. J Biol Chem, 274: 15151-15158

Kuge S, Arita M, Murayama A, Maeta K, Izawa S, Inoue Y, Nomoto A (2001) Regulation of the yeast Yap1p nuclear export signal is mediated by redox signal-induced reversible disulfide bond formation. $\mathrm{Mol}$ Cell Biol, 21: 6139-6150

Kurisaki A, Kurisaki K, Kowanetz M, Sugino H, Yoneda Y, Heldin CH, Moustakas A (2006) The mechanism of nuclear export of Smad3 involves exportin 4 and Ran. Mol Cell Biol, 26: 1318-1332

Kutay U, Bischoff FR, Kostka S, Kraft R, Gorlich D (1997) Export of importin alpha from the nucleus is mediated by a specific nuclear transport factor. Cell, 90: 1061-1071

Kutay U, Lipowsky G, Izaurralde E, Bischoff FR, Schwarzmaier P, Hartmann E, Gorlich D (1998) Identification of a tRNA-specific nuclear export receptor. Mol Cell, 1: 359-369

la Cour T, Gupta R, Rapacki K, Skriver K, Poulsen FM, Brunak S (2003) NESbase version 1.0: a database of nuclear export signals. Nucleic Acids Res, 31: 393-396

la Cour T, Kiemer L, Molgaard A, Gupta R, Skriver K, Brunak S (2004) Analysis and prediction of leucine-rich nuclear export signals. Protein Eng Des Sel, 17: 527-536

Labokha AA, Gradmann S, Frey S, Hulsmann BB, Urlaub H, Baldus M, Gorlich D (2013) Systematic analysis of barrier-forming FG hydrogels from Xenopus nuclear pore complexes. EMBO J, 32: 204-218

Laemmli UK (1970) Cleavage of structural proteins during the assembly of the head of bacteriophage T4. Nature, 227: 680-685

Li W, Yu SW, Kong AN (2006) Nrf2 possesses a redox-sensitive nuclear exporting signal in the Neh5 transactivation domain. J Biol Chem, 281: 27251-27263

Li Y, Yamakita Y, Krug RM (1998) Regulation of a nuclear export signal by an adjacent inhibitory sequence: the effector domain of the influenza virus NS1 protein. Proceedings of the National Academy of Sciences, 95: 4864-4869

Lipowsky G, Bischoff FR, Schwarzmaier P, Kraft R, Kostka S, Hartmann E, Kutay U, Gorlich D (2000) Exportin 4: a mediator of a novel nuclear export pathway in higher eukaryotes. EMBO J, 19: 4362-4371

Madan AP, DeFranco DB (1993) Bidirectional transport of glucocorticoid receptors across the nuclear envelope. Proc Natl Acad Sci U S A, 90: 3588-3592

Mahajan R, Delphin C, Guan T, Gerace L, Melchior F (1997) A small ubiquitin-related polypeptide involved in targeting RanGAP1 to nuclear pore complex protein RanBP2. Cell, 88: 97-107

Mandell RB, Feldherr CM (1990) Identification of two HSP70-related Xenopus oocyte proteins that are capable of recycling across the nuclear envelope. J Cell Biol, 111: 1775-1783

Matsuura Y, Stewart M (2004) Structural basis for the assembly of a nuclear export complex. Nature, 432: 872-877

Matunis MJ, Coutavas E, Blobel G (1996) A novel ubiquitin-like modification modulates the partitioning of the Ran-GTPase-activating protein RanGAP1 between the cytosol and the nuclear pore complex. J Cell Biol, 135: 1457-1470 
McKinsey TA, Zhang CL, Olson EN (2001) Identification of a signal-responsive nuclear export sequence in class II histone deacetylases. Mol Cell Biol, 21: 6312-6321

Melchior F, Paschal B, Evans J, Gerace L (1993a) Inhibition of nuclear protein import by nonhydrolyzable analogues of GTP and identification of the small GTPase Ran/TC4 as an essential transport factor. J Cell Biol, 123: 1649-1659

Melchior F, Weber K, Gerke V (1993b) A functional homologue of the RNA1 gene product in Schizosaccharomyces pombe: purification, biochemical characterization, and identification of a leucine-rich repeat motif. Mol Biol Cell, 4: 569-581

Meyer BE, Malim MH (1994) The HIV-1 Rev trans-activator shuttles between the nucleus and the cytoplasm. Genes Dev, 8: 1538-1547

Milburn MV, Tong L, deVos AM, Brunger A, Yamaizumi Z, Nishimura S, Kim SH (1990) Molecular switch for signal transduction: structural differences between active and inactive forms of protooncogenic ras proteins. Science, 247: 939-945

Mingot JM, Bohnsack MT, Jakle U, Gorlich D (2004) Exportin 7 defines a novel general nuclear export pathway. EMBO J, 23: 3227-3236

Mingot JM, Kostka S, Kraft R, Hartmann E, Gorlich D (2001) Importin 13: a novel mediator of nuclear import and export. EMBO J, 20: 3685-3694

Mohr D, Frey S, Fischer T, Guttler T, Gorlich D (2009) Characterisation of the passive permeability barrier of nuclear pore complexes. EMBO J, 28: 2541-2553

Monecke T, Guttler T, Neumann P, Dickmanns A, Gorlich D, Ficner R (2009) Crystal structure of the nuclear export receptor CRM1 in complex with Snurportin1 and RanGTP. Science, 324: 1087-1091

Monecke T, Haselbach D, Voss B, Russek A, Neumann P, Thomson E, Hurt E, Zachariae U, Stark H, Grubmuller H, Dickmanns A, Ficner R (2013) Structural basis for cooperativity of CRM1 export complex formation. Proc Nat/ Acad Sci U S A, 110: 960-965

Mosammaparast N, Jackson KR, Guo Y, Brame CJ, Shabanowitz J, Hunt DF, Pemberton LF (2001) Nuclear import of histone $\mathrm{H} 2 \mathrm{~A}$ and $\mathrm{H} 2 \mathrm{~B}$ is mediated by a network of karyopherins. J Cell Biol, 153: 251-262

Muhlhausser P, Muller EC, Otto A, Kutay U (2001) Multiple pathways contribute to nuclear import of core histones. EMBO Rep, 2: 690-696

Mullis K, Faloona F, Scharf S, Saiki R, Horn G, Erlich H (1986) Specific enzymatic amplification of DNA in vitro: the polymerase chain reaction. Cold Spring Harb Symp Quant Biol, 51 Pt 1: 263-273

Munoz-Fontela C, Collado M, Rodriguez E, Garcia MA, Alvarez-Barrientos A, Arroyo J, Nombela C, Rivas C (2005) Identification of a nuclear export signal in the KSHV latent protein LANA2 mediating its export from the nucleus. Exp Cell Res, 311: 96-105

Nachury MV, Weis K (1999) The direction of transport through the nuclear pore can be inverted. Proc Natl Acad Sci U S A, 96: 9622-9627

Nagaraj N, Wisniewski JR, Geiger T, Cox J, Kircher M, Kelso J, Paabo S, Mann M (2011) Deep proteome and transcriptome mapping of a human cancer cell line. Mol Syst Biol, 7: 548

Nakielny S, Dreyfuss G (1998) Import and export of the nuclear protein import receptor transportin by a mechanism independent of GTP hydrolysis. Curr Biol, 8: 89-95

Nelson DE, Laman H (2011) A Competitive binding mechanism between Skp1 and exportin 1 (CRM1) controls the localization of a subset of F-box proteins. J Biol Chem, 286: 19804-19815 
Neuhoff V, Arold N, Taube D, Ehrhardt W (1988) Improved staining of proteins in polyacrylamide gels including isoelectric focusing gels with clear background at nanogram sensitivity using Coomassie Brilliant Blue G-250 and R-250. Electrophoresis, 9: 255-262

Neville M, Stutz F, Lee L, Davis LI, Rosbash M (1997) The importin-beta family member Crm1p bridges the interaction between Rev and the nuclear pore complex during nuclear export. Curr Biol, 7: 767-775

Nishi K, Yoshida M, Fujiwara D, Nishikawa M, Horinouchi S, Beppu T (1994) Leptomycin B targets a regulatory cascade of $\mathrm{crm} 1$, a fission yeast nuclear protein, involved in control of higher order chromosome structure and gene expression. J Biol Chem, 269: 6320-6324

O'Neill RE, Talon J, Palese P (1998) The influenza virus NEP (NS2 protein) mediates the nuclear export of viral ribonucleoproteins. EMBO J, 17: 288-296

Ohno M, Segref A, Bachi A, Wilm M, Mattaj IW (2000) PHAX, a mediator of U snRNA nuclear export whose activity is regulated by phosphorylation. Cell, 101: 187-198

Ohtsubo M, Okazaki H, Nishimoto T (1989) The RCC1 protein, a regulator for the onset of chromosome condensation locates in the nucleus and binds to DNA. J Cell Biol, 109: 1389-1397

Oliver FW (1913) Makers of British botany. London: Cambridge University Press,

Ong SE, Mann M (2006) A practical recipe for stable isotope labeling by amino acids in cell culture (SILAC). Nat Protoc, 1: 2650-2660

Ori A, Banterle N, Iskar M, Andres-Pons A, Escher C, Khanh Bui H, Sparks L, Solis-Mezarino V, Rinner O, Bork P, Lemke EA, Beck M (2013) Cell type-specific nuclear pores: a case in point for context-dependent stoichiometry of molecular machines. Mol Syst Biol, 9: 648

Ossareh-Nazari B, Bachelerie F, Dargemont C (1997) Evidence for a role of CRM1 in signal-mediated nuclear protein export. Science, 278: 141-144

Pace CN, Vajdos F, Fee L, Grimsley G, Gray T (1995) How to measure and predict the molar absorption coefficient of a protein. Protein Sci, 4: 2411-2423

Paine PL, Moore LC, Horowitz SB (1975) Nuclear envelope permeability. Nature, 254: 109-114

Pante N, Kann M (2002) Nuclear pore complex is able to transport macromolecules with diameters of about 39 nm. Mol Biol Cell, 13: 425-434

Paraskeva E, Izaurralde E, Bischoff FR, Huber J, Kutay U, Hartmann E, Luhrmann R, Gorlich D (1999) CRM1mediated recycling of snurportin 1 to the cytoplasm. J Cell Biol, 145: 255-264

Partridge JR, Schwartz TU (2009) Crystallographic and biochemical analysis of the Ran-binding zinc finger domain. J Mol Biol, 391: 375-389

Petosa C, Schoehn G, Askjaer P, Bauer U, Moulin M, Steuerwald U, Soler-López M, Baudin F, Mattaj IW, Müller CW (2004) Architecture of CRM1/Exportin1 suggests how cooperativity is achieved during formation of a nuclear export complex. Molecular cell, 16: 761-775

Pinol-Roma S, Dreyfuss G (1992) Shuttling of pre-mRNA binding proteins between nucleus and cytoplasm. Nature, 355: 730-732

Plafker K, Macara IG (2000a) Facilitated nucleocytoplasmic shuttling of the Ran binding protein RanBP1. Mol Cell Biol, 20: 3510-3521

Plafker SM, Macara IG (2000b) Importin-11, a nuclear import receptor for the ubiquitin-conjugating enzyme, UbcM2. EMBO J, 19: 5502-5513

Plafker SM, Macara IG (2002) Ribosomal protein L12 uses a distinct nuclear import pathway mediated by importin 11. Mol Cell Biol, 22: 1266-1275 
Pollard VW, Michael WM, Nakielny S, Siomi MC, Wang F, Dreyfuss G (1996) A novel receptor-mediated nuclear protein import pathway. Cell, 86: 985-994

Radu A, Moore MS, Blobel G (1995) The peptide repeat domain of nucleoporin Nup98 functions as a docking site in transport across the nuclear pore complex. Cell, 81: 215-222

Rawlinson SM, Pryor MJ, Wright PJ, Jans DA (2009) CRM1-mediated nuclear export of dengue virus RNA polymerase NS5 modulates interleukin-8 induction and virus production. J Biol Chem, 284: 1558915597

Reichelt R, Holzenburg A, Buhle EL, Jarnik M, Engel A, Aebi U (1990) Correlation between structure and mass distribution of the nuclear pore complex and of distinct pore complex components. J Cell Biol, 110: $883-894$

Rexach M, Blobel G (1995) Protein import into nuclei: association and dissociation reactions involving transport substrate, transport factors, and nucleoporins. Cell, 83: 683-692

Ribbeck K, Gorlich D (2001) Kinetic analysis of translocation through nuclear pore complexes. EMBO J, 20: $1320-1330$

Ribbeck K, Kutay U, Paraskeva E, Gorlich D (1999) The translocation of transportin-cargo complexes through nuclear pores is independent of both Ran and energy. Curr Biol, 9: 47-50

Ribbeck K, Lipowsky G, Kent HM, Stewart M, Gorlich D (1998) NTF2 mediates nuclear import of Ran. EMBO J, 17: 6587-6598

Richards SA, Lounsbury KM, Carey KL, Macara IG (1996) A nuclear export signal is essential for the cytosolic localization of the Ran binding protein, RanBP1. J Cell Biol, 134: 1157-1168

Rout MP, Blobel G (1993) Isolation of the yeast nuclear pore complex. J Cell Biol, 123: 771-783

Saitoh H, Pu R, Cavenagh M, Dasso M (1997) RanBP2 associates with Ubc9p and a modified form of RanGAP1. Proc Natl Acad Sci U S A, 94: 3736-3741

Sambrook J, Russell DW. (2001) Molecular Cloning: A Laboratory Manual, Third Edition (3 volume set). Cold Spring Harbor Laboratory Press,

Scheffzek K, Klebe C, Fritz-Wolf K, Kabsch W, Wittinghofer A (1995) Crystal structure of the nuclear Rasrelated protein Ran in its GDP-bound form. Nature, 374: 378-381

Schultz J, Milpetz F, Bork P, Ponting CP (1998) SMART, a simple modular architecture research tool: identification of signaling domains. Proc Natl Acad Sci U S A, 95: 5857-5864

Schwartz TU (2005) Modularity within the architecture of the nuclear pore complex. Curr Opin Struct Biol, 15: $221-226$

Schwoebel ED, Talcott B, Cushman I, Moore MS (1998) Ran-dependent signal-mediated nuclear import does not require GTP hydrolysis by Ran. J Biol Chem, 273: 35170-35175

Seewald MJ, Korner C, Wittinghofer A, Vetter IR (2002) RanGAP mediates GTP hydrolysis without an arginine finger. Nature, 415: 662-666

Seimiya H, Sawada H, Muramatsu Y, Shimizu M, Ohko K, Yamane K, Tsuruo T (2000) Involvement of 14-3-3 proteins in nuclear localization of telomerase. The EMBO journal, 19: 2652-2661

Seiser RM, Sundberg AE, Wollam BJ, Zobel-Thropp P, Baldwin K, Spector MD, Lycan DE (2006) Ltv1 is required for efficient nuclear export of the ribosomal small subunit in Saccharomyces cerevisiae. Genetics, 174: 679-691

Smillie DA, Sommerville J (2002) RNA helicase p54 (DDX6) is a shuttling protein involved in nuclear assembly of stored mRNP particles. J Cell Sci, 115: 395-407 
Stevens BJ, Swift H (1966) RNA transport from nucleus to cytoplasm in Chironomus salivary glands. I Cell Biol, 31: 55-77

Stewart M (2003) Structural biology. Nuclear trafficking. Science, 302: 1513-1514

Stommel JM, Marchenko ND, Jimenez GS, Moll UM, Hope TJ, Wahl GM (1999a) A leucine-rich nuclear export signal in the p53 tetramerization domain: regulation of subcellular localization and p53 activity by NES masking. The EMBO journal, 18: 1660-1672

Stommel JM, Marchenko ND, Jimenez GS, Moll UM, Hope TJ, Wahl GM (1999b) A leucine-rich nuclear export signal in the p53 tetramerization domain: regulation of subcellular localization and p53 activity by NES masking. EMBO J, 18: 1660-1672

Stuven T, Hartmann E, Gorlich D (2003) Exportin 6: a novel nuclear export receptor that is specific for profilin.actin complexes. EMBO J, 22: 5928-5940

Subramanian K, Meyer T (1997) Calcium-induced restructuring of nuclear envelope and endoplasmic reticulum calcium stores. Cell, 89: 963-971

Team RD (2012) R: A Language and Environment for Statistical Computing. R Foundation for Statistical Computing, Vienna, Austria, 2007.

Thakar K, Karaca S, Port SA, Urlaub H, Kehlenbach RH (2013) Identification of CRM1-dependent Nuclear Export Cargos Using Quantitative Mass Spectrometry. Mol Cell Proteomics, 12: 664-678

Thomas F, Kutay U (2003) Biogenesis and nuclear export of ribosomal subunits in higher eukaryotes depend on the CRM1 export pathway. J Cell Sci, 116: 2409-2419

Trotta CR, Lund E, Kahan L, Johnson AW, Dahlberg JE (2003) Coordinated nuclear export of 60 S ribosomal subunits and NMD3 in vertebrates. EMBO J, 22: 2841-2851

Truant R, Cullen BR (1999) The arginine-rich domains present in human immunodeficiency virus type 1 Tat and Rev function as direct importin beta-dependent nuclear localization signals. Mol Cell Biol, 19: 1210-1217

Truant R, Kang Y, Cullen BR (1999) The human tap nuclear RNA export factor contains a novel transportindependent nuclear localization signal that lacks nuclear export signal function. $J$ Biol Chem, 274: 32167-32171

van Hengel J, Vanhoenacker P, Staes K, van Roy F (1999) Nuclear localization of the p120(ctn) Armadillo-like catenin is counteracted by a nuclear export signal and by E-cadherin expression. Proc Natl Acad Sci U $S$ A, 96: 7980-7985

Vetter IR, Arndt A, Kutay U, Gorlich D, Wittinghofer A (1999a) Structural view of the Ran-Importin beta interaction at 2.3 A resolution. Cell, 97: 635-646

Vetter IR, Nowak C, Nishimoto T, Kuhlmann J, Wittinghofer A (1999b) Structure of a Ran-binding domain complexed with Ran bound to a GTP analogue: implications for nuclear transport. Nature, 398: 39-46

Vielhaber EL, Duricka D, Ullman KS, Virshup DM (2001) Nuclear export of mammalian PERIOD proteins. J Biol Chem, 276: 45921-45927

Wada A, Fukuda M, Mishima M, Nishida E (1998) Nuclear export of actin: a novel mechanism regulating the subcellular localization of a major cytoskeletal protein. EMBO J, 17: 1635-1641

Wahlberg E, Lendel C, Helgstrand M, Allard P, Dincbas-Renqvist V, Hedqvist A, Berglund H, Nygren PA, Hard $T$ (2003) An affibody in complex with a target protein: structure and coupled folding. Proc Natl Acad Sci U S A, 100: 3185-3190

Walker P, Doenecke D, Kahle J (2009) Importin 13 mediates nuclear import of histone fold-containing chromatin accessibility complex heterodimers. J Biol Chem, 284: 11652-11662 
Watson ML (1959) Further observations on the nuclear envelope of the animal cell. J Biophys Biochem Cytol, 6: 147-156

Weinmann L, Hock J, Ivacevic T, Ohrt T, Mutze J, Schwille P, Kremmer E, Benes V, Urlaub H, Meister G (2009) Importin 8 is a gene silencing factor that targets argonaute proteins to distinct mRNAs. Cell, 136: 496-507

Wen W, Harootunian AT, Adams SR, Feramisco J, Tsien RY, Meinkoth JL, Taylor SS (1994) Heat-stable inhibitors of cAMP-dependent protein kinase carry a nuclear export signal. J Biol Chem, 269: 3221432220

Wen W, Meinkoth JL, Tsien RY, Taylor SS (1995) Identification of a signal for rapid export of proteins from the nucleus. Cell, 82: 463-473

Whittaker GR, Helenius A (1998) Nuclear import and export of viruses and virus genomes. Virology, 246: 123

Wilm M (2009) Quantitative proteomics in biological research. Proteomics, 9: 4590-4605

Xu D, Grishin NV, Chook YM (2012a) NESdb: a database of NES-containing CRM1 cargos. Mol Biol Cell,

Xu D, Farmer A, Collett G, Grishin NV, Chook YM (2012b) Sequence and structural analyses of nuclear export signals in the NESdb database. Molecular biology of the cell,

Yan C, Lee LH, Davis LI (1998) Crm1p mediates regulated nuclear export of a yeast AP-1-like transcription factor. $E M B O$ J, 17: 7416-7429

Yang J, Bardes ES, Moore JD, Brennan J, Powers MA, Kornbluth S (1998) Control of cyclin B1 localization through regulated binding of the nuclear export factor CRM1. Genes Dev, 12: 2131-2143

Yang JM, Wang AH (2004) Engineering a thermostable protein with two DNA-binding domains using the hyperthermophile protein Sac7d. J Biomol Struct Dyn, 21: 513-526

Yokoyama N, Hayashi N, Seki T, Pante N, Ohba T, Nishii K, Kuma K, Hayashida T, Miyata T, Aebi U, et a (1995) A giant nucleopore protein that binds Ran/TC4. Nature, 376: 184-188

Zeitler B, Weis K (2004) The FG-repeat asymmetry of the nuclear pore complex is dispensable for bulk nucleocytoplasmic transport in vivo. J Cell Biol, 167: 583-590

Zhang Y, Xiong Y (2001) A p53 amino-terminal nuclear export signal inhibited by DNA damage-induced phosphorylation. Science, 292: 1910-1915 